UCRL-ID-114972-6

\title{
SERS Internship Spring 1996 Abstracts and Research Papers
}

\author{
Coordinator: \\ Beverly Williams
}

July 1996

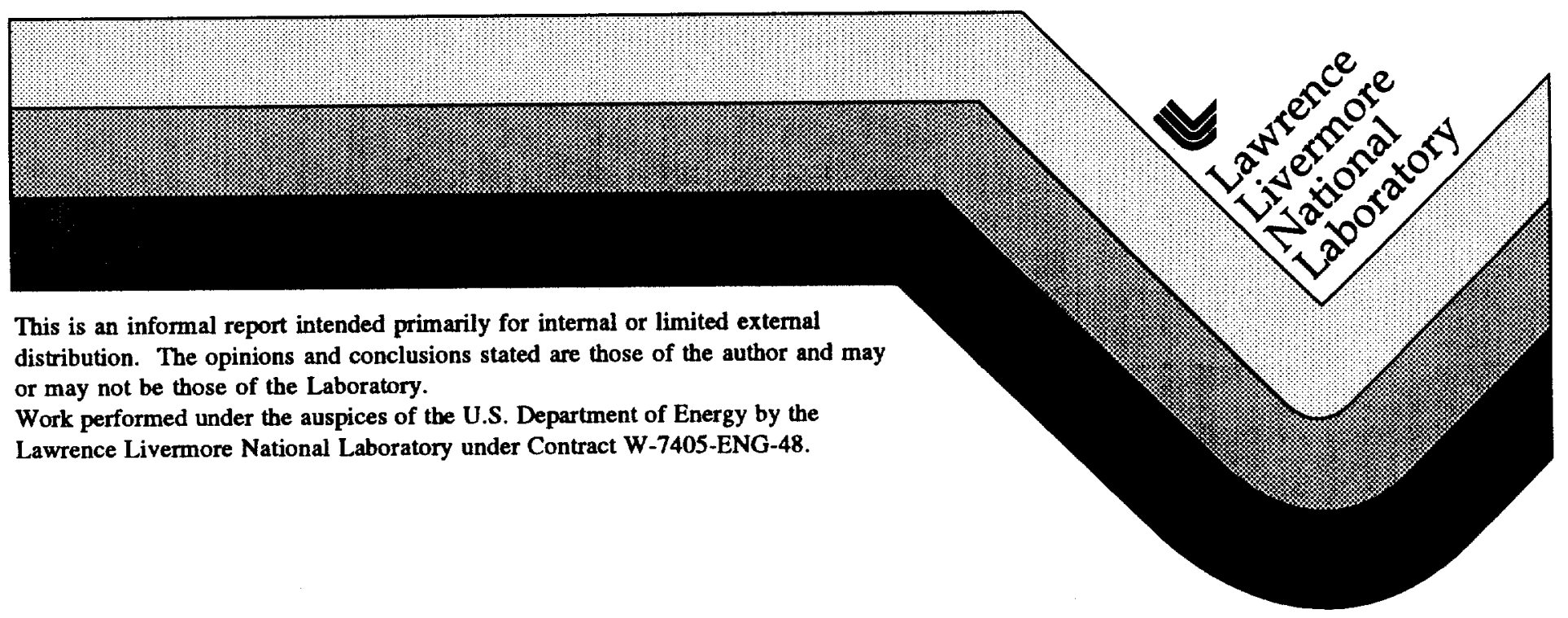




\section{DISCLAIMER}

This document was prepared as an account of work sponsored by an agency of the United States Government. Neither the United States Government nor the University of California nor any of their employees, makes any warranty, express or implied, or assumes any legal liability or responsibility for the accuracy, completeness, or usefulness of any information, apparatus, product, or process disclosed, or represents that its use would not infringe privately owned rights. Reference herein to any specific commercial product, process, or service by trade name, trademark, manufacturer, or otherwise, does not necessarily constitute or imply its endorsement, recommendation, or favoring by the United States Government or the University of California. The views and opinions of authors expressed herein do not necessarily state or reflect those of the United States Government or the University of California, and shall not be used for advertising or product endorsement purposes.

This report has been reproduced directly from the best available copy.

Available to DOE and DOE contractors from the Office of Scientific and Technical Information

P.O. Box 62, Oak Ridge, TN 37831

Prices available from (615) 576-8401, FTS 626-8401

Available to the public from the

National Technical Information Service

U.S. Department of Commerce

5285 Port Royal Rd.,

Springfield, VA 22161 
SCIENCE \& ENGINEERING

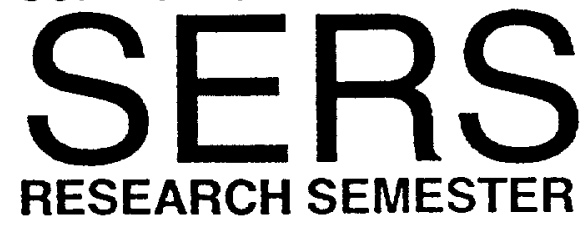

Lawrence Livermore National Laboratory

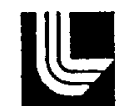

Spring 1996

ABSTRACTS

FOR

\section{STUDENT SYMPOSIUM}

May 2 - 3, 1996

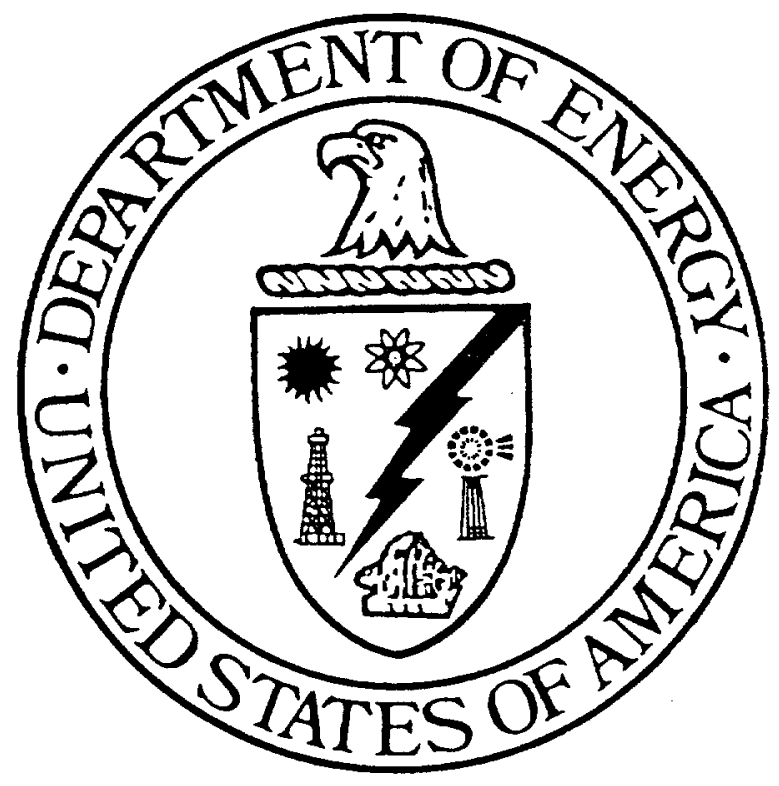

Sponsored by:

The U.S. Department of Energy Office of Energy Research 


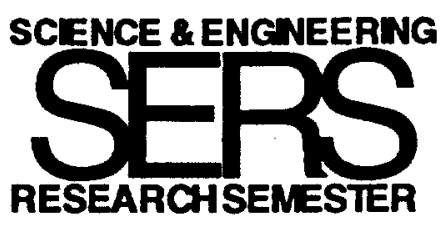

Student Name/Title

Page

Bartels, Randy 1

$\mathrm{ER}^{3}+$ : YAG Superlaser Development

deRopp, Katherine

LLNL's Internal Discharge Limits Revision

Dickenson, Eric

Transport in Porous Media

Dillon, Chris

DNA Fragment Location Program

Drake, Kimberly.

Teaching Science Using an Interactive, Web-based Traffic

Simulation

Dunlop, Amy

Development of ${ }^{59} \mathrm{Ni}$ Accelerator Mass Spectrometry

Capability and Progress Towards Biomedical Tracer

Applications

Helms, Xerxes T.

Inverse Kinematic Program for a Robotic Manipulator

Johnson, Lantz

Temperature Dependence of NiO/NiFe Bilayers for Giant

Magnetoresistance Spin-Valves

Lewis, Stacy

The Development of Nuclear Weapons

Liberatore, Christine 10

Synthesis of a Cleavable Dibiotinyl Linker Molecule 


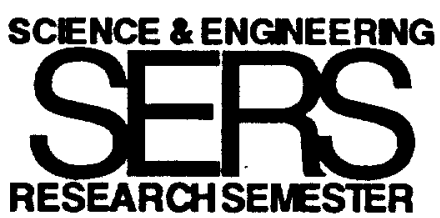

Student Name/Title

\section{Page}

Lilleodden, Erica

Probing the Mechanical Properties of Biological Tissue

Manz, Brian.

Retardation and Diffusion of VOCs in Ground Water

Meissner, Susanne.......................................................................13

Evaluation of a New Model for the Prediction of Skin Uptake

Nguyen, Tri. 14

Conversion of Character-Based Interface to Graphical Interface for ESIS

Pearlman, Jonathan 15

Developing a Finite Element Model for the Carpometacarpal Joint of the Thumb

Rivera, Angel 16

Testing Candidate Alloys for Resistance to Microbial Induced Corrosion

Sparks, James. 17

Isolation of Full-Length cDNA's Using the System GeneTrapper

Wells, Louise 18

A SWMM Model of the Storm Drain System at LLNL 



\title{
Er3+.YAG Superlaser Development
}

\author{
Randy Bartels \\ Oklahoma State University \\ Lasers - ICF
}

\begin{abstract}
A TIR bounce, folded-resonator erbium YAG (Er3+:YAG) solid-state laser was studied to understand how to build a higher average power laser. To understand the factors limiting the power output of the laser we studied thermal lensing and compared results to thermal model calculations performed spectroscopic studies to determine optimal erbium doping level and pump source wavelength developed a kinetics model to verify our understarding of the dynamics of the laser. The thermal lensing studies showed modification of the laser crystal geometry and cooling would reduce thermal lensing and laser crystal temperature. Spectroscopic studies showed the pump diode wavelength used in the current laser design is nearly optimal and losses increase significantly after an erbium concentration of $30 \%$. A new laser system was designed and parts for its construction were ordered. The new laser promises to provide a significant increase in average output power and be more mechanically robust.
\end{abstract}




\title{
LLNLS Internal Discharge Limits Revision
}

\author{
Katherine de Ropp \\ University of Tennessee \\ Environmental Protection Division/ORAD
}

\begin{abstract}
The objective of this study is to clearly identify technically-based internal discharge limits for LLNL's Wastewater Policy Document revision. In order to accurately develop LLNL's internal limits, the different treatment processes at the Livermore Water Reclamation Plant (LWRP) were evaluated. PRELIM modeling software was used to calculate the LWRP's allowable headworks loading. The unallocated portion was then determined, applied to LLNL according to their flow, and compared with current discharge limits. The technical-basis of the current building 196 (B196) limits and internal limits were reviewed. LLNL's internal discharge sources were also evaluated and a mass loading was determined. Mass-balance calculations were performed for these internal discharges and compared with B196 calculated discharge loadings to obtain a percent difference.

The net result is that LLNL is not using their available loading capacity for metals; and LWRP's treatment capabilities are underutilized. The economic savings which could result in reduced wastewater associated costs are currently being evaluated. An Excel model is being developed using LLNL's internal sources to calculate appropriate internal discharge guidelines for the sanitary sewer. The model will improve wastewater management of process discharges and calculate their loading impact on the LWRP.
\end{abstract}




\title{
Transport in Porous Media
}

\author{
Eric Dickenson \\ University of California, Davis \\ Health \& Ecological Assessment
}

\begin{abstract}
A novel nonintrusive fluorescence imaging technique is used to study microscopic transport within porous media. The system consists of a column packed with heterogeneous-transparent particles and a refractive indexmatched aqueous fluid seeded with fluorescent tracer particles or an organic dye. The flow through the column is illuminated by a planar sheet of laser beam and details of flow and transport through the porous regions can be observed microscopically and qualitative and quantitative transport information can be obtained. Various geometric, flow, and concentration quantities can be determined over a three dimensional volume within the column. The quantities include local and volumetrically averaged porosities, velocity and concentration fields, microscopic and volumetrically averaged dispersive fluxes and the dispersion coefficient. The qualitative and quantitative results will provide a better understanding for modeling contaminant transport in porous media.
\end{abstract}




\author{
Chris Dillon \\ Adams State College \\ Human Genome
}

\begin{abstract}
The Human Genome Project is an international effort to find and characterize all genes in humans that encode the basis for all hereditary diseases. Part of characterizing genes is determining which chromosome they are on. The purpose of my project was to develop a program to find out if a gene has already mapped to a chromosome. So, the first thing I did was gather a pool of mapped genes from various sources. Then, I developed a program to help biologists find other fragments of DNA that might overlap with one of the mapped genes. I found that many genes could be mapped without having to do an actual experiment through the use of the computer. I concluded from this that before actual experiments are performed to determine which chromosome a gene is on, the gene should be tested by my program to determine if it needs to be mapped.
\end{abstract}




\title{
Teaching Science Using an Interactive, Web-based

\author{
Kimberly J. Drake \\ Montclair State University \\ Education Program
}

\begin{abstract}
The purpose of this project is to determine an internet-based method to teach mathematics and science. After evaluating possible options, we decided to develop an interactive, traffic simulation which will be accessible from the World Wide Web. At this time, a basic simulation is available along with supporting educational Web pages. In the future, a more enhanced version of the simulation will be available.
\end{abstract}




\title{
Development of 59Ni Accelerator Mass Spectrometry Capability and Progress Towards Bíomedical Tracer Applications
}

\author{
Amy Dunlop \\ Harvard University \\ Center for Accelerator Mass Spectrometry
}

\begin{abstract}
Studies show that exposure to elevated levels of nickel can lead to cancer and other health conditions. Other research suggests the role of nickel as a catalyst in certain biochemical pathways. Accelerator mass spectrometry (AMS) is an ultrasensitive measurement technique for detecting rare, longlived radioisotopes. Because ${ }^{59} \mathrm{Ni}$ is rare in nature, has a long half-life $\left(\mathrm{t}_{1 / 2}=\right.$ $100000 \mathrm{y}$ ) and is able to be separated from its Co isobar, ${ }^{59} \mathrm{Ni}$ tracing with AMS is a possible alternative to the current nickel tracing method, liquid scintillation counting of $63 \mathrm{Ni}\left(\mathrm{t}_{1 / 2}=100 \mathrm{y}\right)$. Furthermore, ${ }^{59} \mathrm{Ni}$ AMS requires 1000 less activity than current methods. Expanding on previous $63 \mathrm{Ni}$ AMS research, we have developed methods for detecting ${ }^{59} \mathrm{Ni}$ with a sensitivity of $\pm 3 \times 10^{7}$ atoms. For biomedical tracing, activity levels and current ${ }^{59} \mathrm{Ni}$ AMS sensitivity imply the ability to measure effects of lower, more biologically relevant doses. At present, we are doing experiments to confirm the feasibility of quantitative extraction of nickel from the tissues of rats that have been dosed with known amounts of ${ }^{9} \mathrm{Ni}$.
\end{abstract}




\title{
Inverse Kinematic Program for a Robotic Manipulator:
}

\author{
Xerxes T. Helms \\ California State University, Sacramento \\ Electronics Engineering/Engineering Research Division
}

\begin{abstract}
The objective of this project is to develop a program that can solve the inverse kinematic equations for a high precision redundant robotic manipulator system. The program uses plane geometry in addition to matrix mathematics to solve the manipulator's equations. The program will calculate the joint angles for a wide range of manipulator positions and produce a graphical representation of the manipulator's position. Using the program with its specifically designed manipulator any operator can analyze the manipulator's position and develop specialized maneuvers.
\end{abstract}




\title{
Temperature Dependence of NiO/NiFe Bilayers for Giant Magnetoresistance Spin-Valves
}

\author{
Lantz Johnson \\ Kent State University \\ Materials Science \& Technology
}

\begin{abstract}
Determine the temperature dependence of $\mathrm{Hc}$ and $\mathrm{He}$ in $\mathrm{NiO} / \mathrm{NiFe}$ thin film bilayers. Films were grown using IBS method and magnetic measurements were made with a VSM equipped with a high temperature oven. A graph showing an approximate linear relationship between the temperature and $\mathrm{Hc}$ and $\mathrm{He}$. Room temperature value of $\mathrm{He}$ can be use to determine $\mathrm{Tb}$ for the sample. In films where no exchange filed was observed a greater value of $\mathrm{Hc}$ was measured whose temperature dependence was similar to that of films showing an $\mathrm{He}>0$. This film also showed a reduced value of $\mathrm{Tb}$ which may be linked to the interfacial properties of the sample.
\end{abstract}




The Development of Nuclear Weap ons

\author{
Stacy Lewis \\ Spellman College \\ Defense \& Nuclear Technologies
}

\begin{abstract}
This paper discusses the development of nuclear weapons and will be used as background information for an interdisciplinary learning module on nuclear weapon nonproliferation. The material gathered from several dozen references is divided into two sections:

(1) pre-WWII (1895 - 1939) and WWII (1939 -1945)

(2) post-WWII (1945 - present).

From the research conducted, it was determined that nuclear weapons developed as a result of:

*historical events

*technological advancements

Upon reading this paper, the teacher will have a clearer understanding of the development of nuclear weapons which will aid in instructing students on nuclear nonproliferation.
\end{abstract}


Synthesis of a Cleavable Dibiotinyl Linker Molecule

\author{
Christine Liberatore \\ Eastern Illinois University \\ Health \& Ecological Assessment
}

\begin{abstract}
The purpose of this project is to synthesize a cleavable, dibiotinyl linker molecule. The proposed synthesis was tested and then systematically altered in attempt to obtain the desired molecule. The first step of the synthesis appears to yield the intermediate molecule. However, attempts to synthesize the product from the intermediate have been unsuccessful due to solvent interactions with the reagents, catalysts, and the products. The testing of solvents is still ongoing. In conclusion, due to these interactions, the proposed synthesis may be abandoned and a new method explored.
\end{abstract}




\title{
Probing the Mechanical Properties of Biological Tissue
}

\author{
Erica Lilleodden \\ University of Minnesota \\ Chemistry \& Materials Science
}

\begin{abstract}
Nanoindentation techniques have proven to be an important method for the determination of mechanical properties of very thin films and surfaces. However, such techniques have been difficult to employ for the study of very compliant, heterogeneous materials in an aqueous environment. Using modified nanoindentation techniques we have measured local, in vitro, mechanical properties of healthy and calcified femoral artery tissue, and demineralized dentin. Using Sneddon's model for linear elasticity as an upper bound for the elastic modulus, and a three element model for viscoelasticity the load vs displacement curves generated in these experiments have been analyzed.
\end{abstract}




\title{
Retardation and Diffusion of VOCs in Ground Water
}

\author{
Brian Manz \\ University of Missouri \\ Environmental Protection Division/ERD
}

\begin{abstract}
The aquifers beneath the LLNL site have been contaminated by volatile organic compounds (VOCs) since World War II. Presently, redmediation is underway to prevent the spread of the contaminants into nearby municipal wells. The current "pump and treat" method seems to have control over the plume but will require decades to completely remove the contamination.

The objective of this research is to develop a better understanding of the physical and chemical processes retarding the VOCs and impeding the cleanup. This study will measure the degrees of retardation of various VOCs, determine if velocity differences of a simulated cleanup affects the degree of retardation, and measure the apparent diffusion of the respective VOCs into different aquifer materials.
\end{abstract}




\title{
Evaluation of a New Model for the Prediction of Skin Uptake
}

\author{
Susanne Meissner \\ California Polytechnic Institute, San Luis Obispo \\ Health \& Ecological Assessment
}

\begin{abstract}
The primary goal of this research project is to determine the uptake rate of chemicals into the skin from three environmental media, water, soil and indoor dust. The U.S. Environmental Protection Agency (EPA) has proposed a dermal uptake model that predicts higher uptake for certain chemicals than previously estimated. Our study focuses on short-term, low level exposure to water contaminants that are comparable to actual environmental exposures. In previous studies such an approach was not achievable due to the lack of sensitive equipment required for measurements on this low of a scale. With the use of Accelerator Mass Spectrometry we have the sensitivity to detect minute amounts of radiolabeled chemical in skin samples following exposures to chemicals at parts per billion $(\mu \mathrm{g} / \mathrm{L})$ concentrations. Using time points from 1 minute to $60 \mathrm{~min}$, we will produce uptake curves from which kinetic parameters can be estimated. These results will be incorporated into the existing EPA model so a more realistic approach to setting contamination standards can be reached.
\end{abstract}




\title{
Conversion of Character-Based Interface to Graphical \\ Interface for ESIS
}

\author{
Tri Nguyen \\ Loyola Marymount University \\ Computations/Computer Applications Science \& Engineering
}

\begin{abstract}
ESIS is an information system for tracking manpower, work orders, and cost accounts for the Electronic Services and Electronic Manufacturing departments. My responsibilities for ESIS are: to fix any errors and crashes, to manage the configurations, to provide documentation about the system, to convert the character-based interface to a graphical interface, and to merge two databases. In particular, this paper will focus on the conversion of a character-based interface to a graphical interface. At the present time, only two modules of ESIS have been converted. After the current bugs and errors in other parts of ESIS have fixed, then the conversion process will continue.
\end{abstract}




\title{
\% Deveroping a Finite Element nodel for the Carpometacarpal Joint of the Thumb
}

\author{
Jonathan Pearlman \\ Northern Arizona University \\ Mechanical Engineering/NTED
}

\begin{abstract}
Developing a finite element model of the carpometacarpal (CMC) joint of the thumb provides essential information for designing anatomically correct and reliable prosthetic joint implants. Using CT scan data of a cadaver hand, a finite element model was developed of the metacarpal and trapezium bones. Finite element analysis results of joint loading will provide biomechanic researchers the guidelines to follow in designing a more reliable joint implant.
\end{abstract}




\title{
Testing Candidate Alloys for Resistance to Microbial Induced Corrosion
}

\author{
Angel Rivera \\ Turabo University \\ Earth Sciences Department
}

\begin{abstract}
The growth of native and introduced bacteria in the Yucca Mountain repository site may jeopardize the integrity of geologic nuclear waste disposal facilities by directly promoting the corrosion of repository components.

Therefore demands a characterization of microbial effects on the repository materials. We investigate the presence of bacteria in samples retrieved from the repository site. These were screened for microbial activities associated with Microbial Induced Corrosion (MIC):

- Acid production

- Sulfate reduction and production of hydrogen sulfide

- Iron oxidation

- Generation of exopolysaccharides (biofilm "slime")

Acid production was found to be highly dependent on the presence of glucose in the media. Glucose-affected alterations of $\mathrm{pH}$ may be reduced by the presence of peptone. As a result of these activities quantification in terms of rate, extent, and correlation to associated growth rates, are being performed. These studies indicate the conditions requisite for metal corrosion by native repository bacteria. They also indicate ideal conditions for accelerated testing of candidates alloys for their resistance to $\mathrm{MIC}$.
\end{abstract}




\title{
Isolation of full Tength CDNA's using the System
}

GeneTrapper

\author{
James Sparks \\ Grambling State University \\ Human Genome
}

\begin{abstract}
The Purpose of this research is too isolate full-length cDNA's on chromosome 19. Currently, We are involved in the isolation of a human Neurocan cDNA. In this retrieval of human Neurocan we used a variety of techniques to isolate the full-length cDNA. They include:

* Northern Blots - determine the mRNA length.

* GeneTrapper - isolate positive clone with cDNA inserts.

* Mini-preps - removal and purification of DNA.

* Restriction digest - enzymatic cutting of DNA to determine cDNA insert Length.

Using the techniques of GeneTrapper along with the conventional methods, we were able to isolate full-length cDNA of human Neurocan of $5.1 \mathrm{~kb}$. Northern blotting showed the cDNA to be approximately $6.8 \mathrm{~kb}$. A new round of primers will be designed to the most recent 5 ' sequence of the Neurocan cDNA.
\end{abstract}




\title{
A SWMM Model of the Storm Brain System at LLNL
}

\author{
Louise Wells \\ Massachusetts Institute of Technology \\ Environmental Protection Division/ORAD
}

\begin{abstract}
The objective of this project is to create a working model of the storm drain system at Lawrence Livermore National Laboratory (LLNL) using the USEPA Stormwater Management Model (SWMM). Once complete, the model will aid in storm water monitoring by creating plots of flow and pollutant loading versus time at different points in the storm drain system. Half of the work on this model has been invested in creating an updated map of the storm drain system, which is necessary for completion of the model, and the other half has been put into setting up the model. Currently, the SWMM model is still under development and is set up to receive more data about the storm drain system as it becomes available and the updated storm drain map is not completed. Nevertheless, the model has been used to analyze a theoretical storm event and to estimate flow rates and the time required for flow to cross the site.
\end{abstract}




\title{
$\mathrm{Er}^{3+}:$ YAG Superlaser Development
}

\author{
Randy Bartels \\ Science and Engineering Research Semester \\ Lasers and Scientific Applications Division \\ Ray Beach, Ralph Page, Steve Sutton, Larry Furu, Steven Payne, and John LaSala \\ Lawrence Livermore National Laboratory
}

\begin{abstract}
A TIR bounce, folded-resonator erbium YAG $\left(\mathrm{Er}^{3+}: \mathrm{YAG}\right)$ solid-state laser was studied to understand how to build a higher average power laser.
\end{abstract}

To understand the factors limiting the power output of the laser we

- Studied thermal lensing and compared results to thermal model calculations

- Performed spectroscopic studies to determine optimal erbium doping level and pump source wavelength

- Developed a kinetics model to verify our understanding of the dynamics of the laser

The thermal lensing studies showed modification of the laser crystal geometry and cooling would reduce thermal lensing and laser crystal temperature. Spectroscopic studies showed the pump diode wavelength used in the current laser design is nearly optimal and losses increase significantly after an erbium concentration of $30 \%$.

A new laser system was designed and parts for its construction were ordered. The new laser promises to provide a significant increase in average output power and be more mechanically robust.

\section{Introduction}

$\mathrm{Er}^{3+}$ :YAG lasers can be made to lase at a wavelength of $2.936 \mu \mathrm{m}$. This wavelength occurs near a hole in atmospheric transmission suggesting possible air communication uses. In addition, the transition is near a water absorption peak hinting at possible medical applications as well.

The first reports of lasers being built using $\mathrm{Er}^{3+}: \mathrm{YAG}$ were published by Russian scientists Prokhorov et $\mathrm{al}^{1,2}$. They reported a operation of a flash lamp pumped $\mathrm{Er}^{3+}:$ YAG laser. The upper level of the $2.936 \mu \mathrm{m}$ transition has much faster lifetime than the lower level 
indicating population inversion would be impossible to obtain, however there is a crossrelaxation mechanism that allows lasing.

$\mathrm{An}^{\mathrm{E}} \mathrm{r}^{3+}$ :YAG laser developed at LLNL in 1993 to communicate with satellites by operating at a wavelength corresponding to a hole in atmospheric transmission, as shown in figure 1 , with some limited tunability range. These goals were rather quickly accomplished and a laser was developed in a period of about 9 months. The program was so successful, the lasers were put into use while still in prototype stages, before the laser was well understood. It is now desired to improve the $\mathrm{Er}^{3+}$ :YAG laser design to produce a higher average power laser with a good quality beam. In order to accomplish this task, the laser must be better understood so we can understand what is causing the problems that inhibit the current laser performance and how we can modify the laser design to correct for those problems.

\section{Current Laser Design and Problems}

The current laser design, shown in figure 2 , utilizes a diode laser array side pumped geometry. A plano-concave resonator is with a $5 \mathrm{~cm}$ radius of curvature high located 33 $\mathrm{mm}$ from one crystal face and a flat output coupler with a transmission of $99 \%$ located 7 $\mathrm{mm}$ from the other face. The cavity is aligned where the beam passes through the crystal and makes a TIR ( total internal reflection) bounce in the center of the erbium YAG crystal.

A single TIR bounce cavity is advantageous because it can yield a higher gain per pass than a straight through cavity ${ }^{3}$. The pump light is preferentially absorbed at the surface of the crystal pump face, therefore, the largest gain region is at the surface of the pump face. The more time the internal laser beam spends close to the pump face, the higher the gain per pass of the laser will be.

When the internal laser beam comes too close to the pump face, the beam will experience diffraction losses due to clipping at the edge of the laser crystal face. This will reduce the overall gain per pass of the laser. A balance must be made between the high gain achieved by closer proximity to the pump face and resulting difffractive losses.

The laser crystal geometry is designed to cause a TIR bounce in the center of the crystal while the laser beam enters and exits the crystal at Brewster's angle. Entering and exiting at Brewster's angle should eliminate reflection losses for the horizontal plane of polarization. The laser will not enter and exit at exactly Brewster's angle, so some reflective (Fresnel) losses will be encountered.

The crystal is mounted in a water cooled copper chill bar. Indium was wrapped on the three non-optical surfaces to provide good thermal contact with the copper chill bars. However, careful inspection of the mounted crystal indicates good thermal contact exists only on the top and bottom of the crystal. The pump diodes and the crystal holder are 
cooled separately. They are mounted on a stainless-steel breadboard, and are still thermally connected.

\section{Pump Source}

To operate a laser, you must supply power to a gain media that will be converted into laser light at a specific frequency. The gain media used in this laser is a $50 \%$ doped erbium YAG crystal. A 5-bar InGaAs laser diode array supplies that is focused onto the face of the crystal.

The diode array use microchannel cooling technology developed at $\mathrm{LLNL}^{4}$. The array, shown in figure 3 , consists of microchannel cooled packages mounted together on a block with cooling water and electrical connections. Diode bars are grown using an MOCVD reactor. The wavelength is chosen by specifying the materials and size of quantum wells. The bars are mounted on a microchannel silicon layer. A cylindrical fiber microlens is mounted on the microchannel package to focus the highly diverging light emitted by the diode bars.

The diode light is coupled into the side face of the laser crystal. The diode bar light is focused by microlenses mounted on the diode packages. Two cylindrical lenses then focus the light into a thin stripe on the side face of the $\mathrm{Er}^{3+}$ :YAG crystal, as demonstrated in figure 4 .

Laser diodes used in diode bar strips have small junctions and therefore small heat capacity. A change in diode junction temperature will shift the wavelength of operation of the diodes During the course of a pulse of diode light, heat is deposited in the diode junction from the electrical power supplied to the diode array. As a result the peak wavelength output from the diode array shifts, chirps, during the duration of the diode pulse.

The combined, time-averaged spectrum of the pump diodes was measured by placing a small integrating sphere near the diode output. An optical fiber was routed from the integrating sphere, where measured the intensity spectra of the diode light during the pump pulse. The diode light was averaged spectrally in time, as a consequence, the chirping, or wavelength shift of the diodes was lost.

Figure 5 shows time-average pump diode spectra obtained overlaid with the absorption spectra of $50 \%$ doped $\mathrm{Er}^{3+}:$ YAG. A comparison of the pump spectra and the absorption spectra of the $\mathrm{Er}^{3+}$ :YAG shows the peak of the diode output occurs slightly away from a nearby absorption peak in erbium YAG.

The question arose as to whether tuning the peak diode wavelength (via laser diode junction temperature) would increase the absorption of the pump light in the crystal. To determine this, we first needed a measure of the average spectral absorption in the crystal. 
We defined an effective, spectrally averaged, absorption $\alpha_{e f f}$ as

$$
\alpha_{e f f}=\frac{\int_{\lambda} \alpha(\lambda) G(\lambda) \exp (-\alpha(\lambda) z) d \lambda}{\int_{\lambda} G(\lambda) \exp (-\alpha(\lambda) z) d \lambda}
$$

where $\alpha(\lambda)$ is the spectral distribution of $\mathrm{Er}^{3+}: \mathrm{YAG}$ and $\mathrm{G}(\lambda)$ is the spectral distribution of the pump diodes

Equation (1) was implemented using the digitized spectral data and a PC to numerically integrate the data. Figure 6 shows the results of the calculation. The effective absorption monotonicly decays inside the erbium YAG crystal.

If the diodes are tuned to different peak wavelengths, we would expect the effective absorption to vary. A maximum value indicates the best overlap between the pump diodes and the absorption of erbium YAG.

The effective absorption at the pump face was calculated versus a shift in the center wavelength (wavelength tuning) of the diode spectra. The results of this calculation are shown in figure 7. The zero point on the $x$-axis represents to measured diode emission peak. Positive values of wavelength shift represent shifting of the diode peak wavelength to higher values while negative values represents a shift of the diode peak wavelength to lower values. There is not as large of a change in the spectrally average effective absorption as one might expect to see, indicating little advantage would be gained by temperature tuning the diodes to a higher wavelength.

Figure 8 shows an experimental setup used to perform a razor blade drag experiment. The detector measures the spatially integrated intensity of the laser beam it is dragged across. The razor blade is moved to a position where all none of the laser light is attenuated. The razor blade is mounted on a micrometer. The distance between the $90 \%$ transmission of the light and $10 \%$ transmission of the light is called the razor blade drag spot size.

A razor blade drag spot size was performed at the pump face of the laser crystal. A spot size of $200 \mu \mathrm{m}$ was measured. The width of the spot size was estimated to be $7 \mathrm{~mm}$.

The pump rate in the crystal tells us the rate at which population is being transferred from a lower energy level to a higher energy level due to some power input to the crystal. An estimated can be obtained by knowing the intensity distribution inside the crystal and the area of the spot on the pump face, where the spatially averaged pump rate can be written as 


$$
\Gamma_{p u m p, a v g}=\frac{P_{0} \alpha_{e f f}(z)}{h l_{d} w} \exp \left(-\int_{0}^{z} \alpha_{e f f}\left(z^{\prime}\right) d z^{\prime}\right)
$$

where

$\alpha_{e f f}(z)$ is the spectrally averaged absorption coefficient computed above

$l_{d}$ is the length of the focus diode stripe

$w$ is the focus height of the diode stripe

$P_{0}$ is the peak pump power at the crystal diode pump face

Using the above expression, a spatially averaged pump rate of $4.64 \mathrm{e}^{2} 3 \mathrm{~cm}^{-3}$ was calculated.

\section{Laser Cross-section Values}

There are two sets of relevant $\mathrm{E}^{3+}$ :YAG cross-section values in the literature. The first values we used in our calculations were numbers obtained from $\mathrm{S}$. Payne et. Al published in $1993^{5}$. At the last Advanced Solid State Laser conference in February of 1996, G. Huber et. Al. Published cross-section values a factor of 7 smaller than values of S. Payne ${ }^{6}$.

S. Payne's cross-section values were obtained by measuring the emission spectra around $2.936 \mu \mathrm{m}$ and correcting it for the lamp source. The florescence intensity was then scaled to cross-section values using ${ }^{7}$

$$
\sigma(v)=\frac{\lambda^{2}}{\tau_{r a d} 8 \pi n^{2}}\left(\frac{\lambda^{2} I(\lambda)}{c \int I(\lambda) d \lambda}\right)
$$

where $\boldsymbol{n}$ is the index of refraction of the material, $\lambda$ is the wavelength, $\mathrm{c}$ is the speed of light, $I(\lambda)$ is the luminescence intensity distribution, and $\tau_{\text {rad }}$ is the radiative lifetime of the upper stark manifold.

The accuracy of the cross-section values is limited by the radiative lifetime value used. For this calculation, a radiative lifetime of $6.4 \mathrm{~ms}^{\text {was }}$ used $^{5}$.

However, for calculations involving the laser, we need both emission and absorption cross-section values. To obtain the absorption cross-sections, we must apply McCumber theory to obtain the absorption cross-section values.

Figure 9 shows a series of stark levels in an upper and a lower energy manifold ${ }^{8}$. The transition from the bottom of the lower manifold to the bottom of the upper manifold is called the zero photon energy, $E_{0}$. The absorption cross-section $\sigma_{\text {abs }}(v)$ values can be 
obtained from a known emission cross-section $\sigma_{\mathrm{ems}}(v)$ distribution by the following transformation ${ }^{5}$

$$
\sigma_{a b s}(v)=\sigma_{e m s}(v) \frac{Z_{u}}{Z_{l}} \exp \left(\frac{h v-E_{0}}{k T}\right)
$$

where the partition function for the upper and lower manifolds $Z_{u}$ and are $Z_{l}$ defined as

$$
Z=\sum_{j} d_{j} \exp \left(\frac{E_{j}}{k T}\right)
$$

and $d_{j}$ is the degeneracy of each stark level in the manifold.

The resulting cross-sections are shown in figure 10 .

\section{Kinetics Modeling}

Laser system have demonstrated interesting dynamic behavior from their earliest construction. The $\mathrm{Er}^{3+}$ :YAG laser demonstrates extremely complex behavior in the form of spiking due to relaxation oscillations and chirping as a result of the complex dynamic interactions exchanged between the various stark transitions. The following kinetics model employs rate equations describing the interacts of the energy levels in $\mathrm{Er}^{3+}: \mathrm{YAG}$.

Figure 11 shows the energy levels of the $\mathrm{Er}^{3+}$ :YAG system. The levels are split into distinct stark levels as shown in figure 9. The laseing transition occurs from the $3 \rightarrow 2$ transition as labeled in the picture, but there are many distinct stark transitions where laseing can occur. Laser output indeed chirps between three different wavelengths. In order to model the chirping, the rate equations were written to track photon densities in individual photon channels.

$$
\begin{aligned}
& \frac{d \phi\left(v_{i}\right)}{d t}=\phi\left(v_{i}\right) \frac{l_{s} c}{l_{c}}\left[n_{3} \sigma_{e m s}\left(v_{i}\right)-n_{2} \sigma_{a b s}\left(v_{i}\right)\right]-\frac{\phi\left(v_{i}\right)}{\tau_{c}} \\
& \frac{d n_{3}}{d t}=\Gamma_{p u m p}-\frac{n_{3}}{\tau_{3}}+\omega_{C R} n_{2}^{2}-\omega_{C R}^{\prime} n_{3}^{2}-\sum_{i}^{n} \phi\left(v_{i}\right) \frac{l_{s} c}{l_{c}}\left[n_{3} \sigma_{e m s}\left(v_{i}\right)-n_{2} \sigma_{a b s}\left(v_{i}\right)\right]
\end{aligned}
$$

$$
\frac{d n_{2}}{d t}=\frac{n_{3}}{\tau_{3}}-\frac{n_{2}}{\tau_{2}}-2 \omega_{C R} n_{2}^{2}+\sum_{i}^{n} \phi\left(v_{i}\right) \frac{l_{s} c}{l_{c}}\left[n_{3} \sigma_{e m s}\left(v_{i}\right)-n_{2} \sigma_{a b s}\left(v_{i}\right)\right]
$$




\begin{tabular}{|c|c|c|c|}
\hline \multicolumn{4}{|c|}{ Kinetics Model Parameters } \\
\hline Symbol & Description & Value & Source \\
\hline$\tau_{3}$ & Lifetime of level $3-{ }^{4} \mathrm{I}_{11 / 2}$ & $100 \mu \mathrm{s}$ & \\
\hline$\tau_{2}$ & Lifetime of level $2-{ }^{4} \mathrm{I}_{13 / 2}$ & & \\
\hline$n_{3}$ & Population density of level $3\left(\mathrm{~cm}^{-3}\right)$ & & \\
\hline$n_{2}$ & Population density of level $2\left(\mathrm{~cm}^{-3}\right)$ & & \\
\hline$\sigma_{\mathrm{ems}}\left(v_{i}\right)$ & $\begin{array}{l}\text { Emission Cross section of the level } 3 \\
\rightarrow \text { level } 2 \text { transition, }{ }^{4} I_{11 / 2} \leftrightarrow{ }^{4} I_{13 / 2} \\
\left(v_{i}\right) \text { represents the individual photon } \\
\text { channels }\end{array}$ & & 1,2 \\
\hline$\sigma_{\mathrm{ats}}\left(v_{i}\right)$ & $\begin{array}{l}\text { Absorption Cross section of the level } \\
3 \rightarrow \text { level } 2 \text { transition, }{ }^{4} I_{11 / 2} \leftrightarrow{ }^{4} I_{13 / 2}\end{array}$ & & 1,2 \\
\hline$l_{s}$ & Length of the $\mathrm{Er}^{3+}: \mathrm{YAG}$ crystal & & \\
\hline$l_{c}$ & Optical length of the resonator & & \\
\hline $\mathrm{c}$ & Speed of light & $3 \mathrm{e} 10(\mathrm{~cm} / \mathrm{s})$ & \\
\hline$\omega_{S T}$ & Cross-relaxation constant & & \\
\hline$\omega_{\mathrm{cr}}$ & Cross-relaxation constant & & \\
\hline$\Gamma_{\text {pump }}$ & $\begin{array}{l}\text { Pump rate from level } 1 \text { to level } 4 \text { - } \\
{ }^{4} I_{15 / 2} \rightarrow{ }^{4} I_{9 / 2}\end{array}$ & & \\
\hline$\phi\left(v_{i}\right)$ & $\begin{array}{l}\text { Photon densities in the individual } \\
\text { channels }\left(\mathrm{cm}^{-3}\right)\end{array}$ & & \\
\hline$\tau_{\mathrm{c}}$ & Cavity lifetime & & \\
\hline
\end{tabular}

Ground-state depletion is a significant reduction in the ground level population density (level 1 in the kinetics model). If ground state depletion effects become large, absorption of the pump light will be reduced.

$$
\Gamma_{p u m p}=\Gamma_{0}\left(1+\frac{n_{3}+n_{2}}{n_{E r^{3+}}}\right)
$$

where $\Gamma_{0}$ is the initial pump rate and $n_{E r}{ }^{3+}$ is the doping density of the erbium in YAG The cavity lifetime $\tau_{\mathrm{c}}$ is computed from geometry and losses in the resonator.

$$
\tau_{c}=-\frac{2 l_{c} / c}{\ln \left(T^{2} R\right)}
$$

where 
$I_{c}$ is the optical path length of the resonator

$c$ is the speed of light

$T$ is the one-way cavity transmission

$\mathrm{R}$ is the reflectivity of the output coupler

The kinetics model equations relate how changes in population densities in the $\mathrm{Er}^{3+}$ :YAG system effect the laser output. In the initial state, before the pump diodes are turned on, the population densities of level 1 , is approximately equal to the erbium doping density. The upper level populations densities $\left(n_{4}, n_{3}, \& n_{2}\right)$ will be approximately zero.

The pump diodes will transfer population from level 1 to level 3 via excited state absorption. Once at level 3, the population can be lost due to decay, stimulated emission, or cross-relaxation. Gain will be created to the $2700 \mathrm{~nm}$ line, that stark transition will bottleneck, and gain will switch to the $2830 \mathrm{~nm}$ transition that will bottleneck and finally the $2936 \mathrm{~nm}$ line will become active.

\section{Linearization of the Kinetics Model Equations}

The kinetics equations were linearized about their steady-state solutions for one photon channel. To see dynamic behavior about the steady-state solution, the eigenvectors of a $3 \times 3$ matrix consisting of the linear set of equations ()$, 0, \&()$ must be solved. The solution to this is known due to a resulting cubic characteristic equation, however the number of terms generated by this solution hinder interpreting any physical meaning into the solution. The kinetics model simulation performed on a computer are more enlightening.

\section{Model Calculations}

The kinetics model equations were solved by using finite differencing techniques using Matlab on a 486 platform. For initial investigations, all 42 stark transitions were tracked in individual photon channels. The cross-sections for the various stark wavelengths were extracted from the $\mathrm{Er}^{3+}: \mathrm{YAG}$ emission data that had been transformed to emission and absorption cross-section values via McCumber theory. When the code including all 42 stark transitions was run, only the three wavelengths observed in the laser output contributed significant photon populations. Further calculations included only the 2700 , $2830, \& 2936 \mathrm{~nm}$ wavelength lines in order to reduce computation time.

Figure 12 shows kinetics model simulations.

- The pump pulse begins at $\mathrm{t}=0$.

- There is a finite time until the first $(2700 \mathrm{~nm})$ wavelength appears, this is referred to as the build-up time. The $2700 \mathrm{~nm}$ line begins decay through a relaxation oscillation until it is quenched by the $2830 \mathrm{~nm}$ line. 
- This time is referred to as the build-up time for the $2830 \mathrm{~nm}$ line. The 2830 line then decays through a relaxation oscillation.

- The $2830 \mathrm{~nm}$ line is quenched by the $2936 \mathrm{~nm}$ line, build-up tome of the 2936 $\mathrm{nm}$ line. The $2936 \mathrm{~nm}$ line then decays through a relaxation oscillation to a steady-state value.

The kinetics model simulation and the laser output, shown in figure 13, qualitatively show similar behavior. Both show a finite build-up time until the first wavelength appears in the output. The laser output then goes through a series of relaxation oscillations as the kinetics model from shorter to longer wavelengths.

Under proper conditions, the laser output produces the 2700,2830 , and $2936 \mathrm{~nm}$ wavelengths. However, a slight change in the pump pulse or laser cavity layout, produced different build-up times and possibly skips a laser output line, such as the $2830 \mathrm{~nm}$ line. These changes in the laser

The kinetics model is a point model, while the laser has action over the three-dimensional pump/laser beam volume. The pump rate is varies three-dimensionally inside the laser crystal. The pump rate used was a spatially averaged value.

An extensive exploration of the parameter space of the kinetics model was performed. Figure 14 shows contour a contour plot of the $2700 \mathrm{~nm}$ build-up time. The cross-sections used in the model to generate figure 14 were cross-sections derived from S. Payne's numbers. The observed buildup time is $15 \mu \mathrm{s}$, so that puts us out of reasonable pump rates and cavity losses in figure 14 . Figure 15 shows the $2700 \mathrm{~nm}$ line buildup time using cross-section values scaled to $G$. Huber's values. The cavity losses and pump rates corresponding to a $15 \mu \mathrm{s}$ buildup time are close to what we expect. This seems to indicate $\mathrm{G}$. Huber's cross-section values may be more accurate.

\section{Thermal Lensing}

Thermal lensing is a major problem standing in the way of scaling the working laser to higher powers. The pump diodes that supply power to the laser crystal deposit heat into the crystal. The heat distribution depends on the spatial shape of the pump pulse, which determines the heat source distribution, the geometry of the crystal, and the cooling arrangement. The heat deposited in the laser crystal produces a change in the index of refraction in the crystal. This change in index of refraction acts like a lens in the laser resonator cavity. If the lensing becomes too large, the cavity will become unstable and the laser will fail.

\section{Thermal Focal Length Approximation}


A first order approximation of the focusing effects in the laser rod can be made. To do this, we assumed a top hat pump pulse and thereby thermal heat source. The foot print of the pump pulse was assumed to be $200 \mu \mathrm{m}$ by $7 \mathrm{~mm}$. An observation of the indium surrounding the rod indicated there was good contact on only the upper and lower surfaces. Therefore, heat was assumed to flow only in the vertical direction, with no horizontal heat flow. The heat flow from the source will be governed by equation (11) ${ }^{7}$.

$$
J=\kappa \frac{\partial T}{\partial x}
$$

where $\mathbf{J}$ is heat flux flowing across the boundary. Assuming a top-hat heat source, we can estimate the thermally induced focal length by

$$
\frac{1}{f_{t h}}=P_{\text {diodes }} \frac{\frac{\partial n}{\partial t}}{w^{2} \kappa}
$$

Equation (12) shows a linear relationship between inverse focal length (dioptric power) and pump power.

Assuming $100 \%$ conversion of pump light into heat, we obtain a focal length of $1.0 \mathrm{~cm}$ from this calculation.

\section{Thermal Lensing Measurements}

Figure 16 shows an experimental setup used to study thermal lensing effects. A Helium Neon $(\mathrm{HeNe})$ laser is telescoped down from a spot size of $500 \mu \mathrm{m}$ to $100 \mu \mathrm{m}$. A razor blade drag was performed at the entrance of the telescope and at the telescope waist to obtain those numbers. The waist of the telescoped beam was placed inside the crystal being studied yielding a collimated beam inside the crystal.

We can quantify the distance over which the beam is well collimated. This distance is known as the Rayleigh range?

$$
d=\frac{\omega_{0}^{2}}{2 \pi \lambda}
$$

A Rayleigh range of $5 \mathrm{~cm}$ was calculated. This means the beam is approximately collimated $5 \mathrm{~cm}$ either side of the focus of the telescoping system. The sample length is $1.5 \mathrm{~cm}$, so the light going through the sample should be approximately collimated. 
A ccd camera was placed $50 \mathrm{~cm}$ from the center of the crystal. The camera imaged the distortion seen by the HeNe probe beam as it passed through the thermal lens present in the crystal. The spot size on the camera was scaled to match the spot size measured at the camera image plane. Using gaussian optics ABCD matrices, focal lengths for the experimental setup were computed.

The first thermal lensing setup used was a square ended straight through measurement. This was chosen in an attempt to deconvolve the effects of the TIR bounce from the effect of thermal lensing on a beam traveling through the crystal. The telescoped beam entered the rod in the center of the end face and traveled through it at a path approximately parallel to the outer surfaces, exiting the opposite end face in the center of the rod. For various current values supplied to the diodes, various spot sizes were obtained from the beam code. They are summarized in table 2 . A second thermal lensing setup employed a single TIR bounce of the probe beam in the square-ended rod. A final experiment was performed on the Brewster angled rob, where the probe beam enters the rod a Brewster's angle and performs a TIR bounce.

Once the thermal focal length studies were performed, we had a list of spot sizes and diode current values. The diode power was estimated from the current values and the induced thermal focal lengths at the various power levels were estimated by calculating the focal lengths that matched the spot size at the ccd camera.

To estimate the induced thermal focal lengths, we assumed a gaussian plane wave entered the telescope and we calculated the spot size at the camera position as a function of the thermally induced focal length. The ABCD matrix describing the system is shown below

$$
\mathbf{M}=\left[\begin{array}{ll}
1 & z \\
0 & 1
\end{array}\right]\left[\begin{array}{cc}
1 & 0 \\
-1 / f_{t h} & 1
\end{array}\right]\left[\begin{array}{cc}
1 & d_{s} \\
0 & 1
\end{array}\right]\left[\begin{array}{cc}
1 & 0 \\
-1 / f_{2} & 1
\end{array}\right]\left[\begin{array}{cc}
1 & f_{1}+f_{2} \\
0 & 1
\end{array}\right]\left[\begin{array}{cc}
1 & 0 \\
-1 / f_{1} & 1
\end{array}\right]
$$

where

$f_{t h}$ is the induced thermal focal length

$d_{S}$ is the distance from the collimating telescope to the center of the sample $z$ is the distance to the ccd camera

The waist at where $z=50 \mathrm{~cm}$ was calculated as a function of focal length. Figure 17 shows those results.

\section{Thermal Gradient Management ${ }^{10}$}

Two dimensional calculations of thermal temperature rises, thermal stress, and thermal lensing were performed on samples shown in figure 18 and figure 19. Table 3 summarizes the properties used in the calculations. 
Figure 18 shows the current crystal geometry and cooling setup. The cooling is assumed to occur only on the top and bottom faces of the crystal, poor thermal contact is assumed on the face opposite of the pump face and not included. Faces that do not have conductive cooling present are assumed to be adiabatic.

Figure 19 shows the an alternative crystal geometry and cooling setup. The $\mathrm{Er}^{3+}: \mathrm{YAG}$ crystal is diffusion bonded to a $0.5 \mathrm{~mm}$ sapphire pedestal on a $2.0 \mathrm{~mm}$ thick sapphire substrate. The pedestal serves to reduce stresses that occur at right angle saphire$\mathrm{Er}^{3+}$ :YAG interfaces. Cooling is performed by placing copper chill bars, water cooled, as close to the sapphire pedestal as possible. Cooling of the $\mathrm{Er}^{3+}: \mathrm{YAG}$ crystal through the sapphire substrate. Faces that do not have conductive cooling present are assumed to be adiabatic.

Figure 20 compares the temperature rise in the two sample geometry's. A lower temperature at the pump face is desired because that will result in higher gain. Geometry $\# 1$ has a far lower temperature at the pump face.

Figure 21 show the radius of curvature inside the sample, which is a measure of the strength of the thermal lensing. Geometry \#1 displays less thermal lensing than does geometry $\# 1$.

\section{Resonator Stability and Mode Overlap}

A stable resonator cavity is one in which a beam performing a round-trip bounce in the cavity exactly repeats itself in that bounce. Using ABCD matrix formalism, we can describe any laser cavity by choosing an reference plane inside the cavity ${ }^{11}$. The matrix $\mathbf{M}$

$$
\mathbf{M}=\left[\begin{array}{ll}
A & B \\
C & D
\end{array}\right]
$$

describing the round-trip beam path in the cavity is written for that reference plane. Since the beam in a stable cavity takes the same path in a round-trip, the beam waist and radius of curvature will be identical for every bounce at a given point. This means the complex curvature $q_{0}$ at the reference plane must be the same before and after a round-trip in the cavity. This property is referred to as self-consistency of the beam. Using the round-trip matrix for a cavity, we can solve for the complex curvature at the reference plane.

$$
q_{0}=\frac{A q_{0}+B}{C q_{0}+D}
$$

For the cavity to be stable, the condition 


$$
\frac{A+D}{2} \mid \leq 1
$$

must be satisfied.

For the existing $\mathrm{Er}^{3+}$ :YAG laser design, the cold-cavity beam waist throughout the laser cavity was computed. Figure 2 shows a diagram of the cavity used in the calculation. A reference plane was chosen at the flat output coupler and the round-trip cavity matrix for the tangential and saggital planes were written as

$$
\begin{aligned}
& M_{T}=M 1 \cdot D 1 \cdot \text { Bout }_{T} \cdot D 2 \cdot \text { Bin }_{T} \cdot D 3 \cdot M 2 \cdot D 3 \\
& \cdot \text { Bout }_{T} \cdot D 2 \cdot \text { Bin }_{T} \cdot D 1 \\
& M_{S}=M 1 \cdot D 1 \cdot \text { Bout }_{S} \cdot D 2 \cdot \text { Bin }_{S} \cdot D 3 \cdot M 2 \cdot D 3 \\
& \cdot \text { Bout }_{S} \cdot D 2 \cdot \text { Bin }_{S} \cdot D 1
\end{aligned}
$$

where

$$
\begin{array}{lll}
M 1=\left(\begin{array}{ll}
1 & 0 \\
0 & 1
\end{array}\right) & D 2=\left(\begin{array}{cc}
1 & d_{2} \\
0 & 1
\end{array}\right) & \text { Bout }_{T}=\left(\begin{array}{cc}
1 / n & 0 \\
0 & n
\end{array}\right) \\
M 2=\left(\begin{array}{cc}
1 & 0 \\
-\frac{2}{R} & 1
\end{array}\right) & D 3=\left(\begin{array}{cc}
1 & d_{3} \\
0 & 1
\end{array}\right) & \operatorname{Bin}_{S}=\left(\begin{array}{cc}
1 & 0 \\
0 & 1 / n
\end{array}\right) \\
D 1=\left(\begin{array}{cc}
1 & d_{1} \\
0 & 1
\end{array}\right) & \operatorname{Bin}_{T}=\left(\begin{array}{cc}
n & 0 \\
0 & 1 / n^{2}
\end{array}\right) & \text { Bout }_{S}=\left(\begin{array}{ll}
1 & 0 \\
0 & n
\end{array}\right)
\end{array}
$$

The cavity is stable which means a beam that enters the round-trip operator will come back out looking exactly the same, that is it will be self-consistent. Imposing self consistency on equations (18) and (19), we obtain complex beam parameters of

at the output coupler. Giving us waist sizes of at the output of the laser.

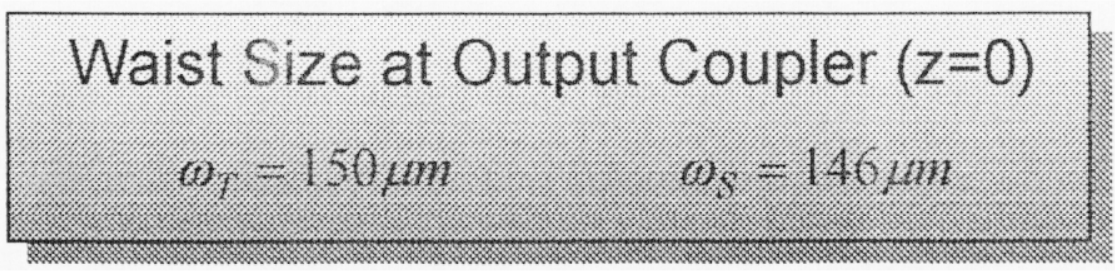


Figure 23 shows the saggital and tangential beam waist inside the laser cavity.

$\mathrm{W}$ pump power is supplied to the laser crystal, some of the power is deposited as heat in the crystal which produces a thermal lens. The thermal lens changes the cavity configurations and a large enough lens can make the laser cavity become unstable and cause it to cease operation.

Inverse focal length, $1 / f$, is called dioptric power. Dioptric power will be proportional to diode power. If we plot the active resonator stability parameters on the stability diagram, we can see it traces out a path along the diagram. With the current cavity configuration, we can see there will be two regions of stability and eventually further increases in dioptric power (diode power) will cause the cavity to remain unstable. 


\section{References}

C. E. Hamilton, L. Furu, R. Beach, R. W. Solarz, and W. F. Krupke, LLNL paper

A. A. Kaminski, A. G. Petrosyan, G. A. Denisenko, T. I Butaeva, A. A. Feorov, and S. E. Sarkisov,

R. Spring and W. Lüthy, J. Appl. Phys, 69 (2), 15 January 1991

J. E. Bernard and A. J. Alcock, Optics Letters, Vol. 18, No. 12, June 15, 1993

M. Pollnau, W. Lüthy, H. P. Wever, T. Jensen, G. Huber, A. Cassanho, H. P. Jenssen, R. A. McFarlane, Optics Letters, Vol. 21, No. 1, January 1, 1996

T. Gensen, G. Huber, and K. Peterman, conference proceedings, Advanced Solid State Lasers 1996

V.I. Zhekov, V. V. Lobachev, T.M. Murina, A.V. Popov, A.M. Prokhorov, and M.I.

Studenikin, Sov. J. Quantum Elec., 19 (6), June 1989

S. A. Payne, L.L. Chase, L. K. Smith, W. L. Kway, and W. F. Krupke, IEEE J. Quantum Elec., Vol. 28, No. 11, November 92 


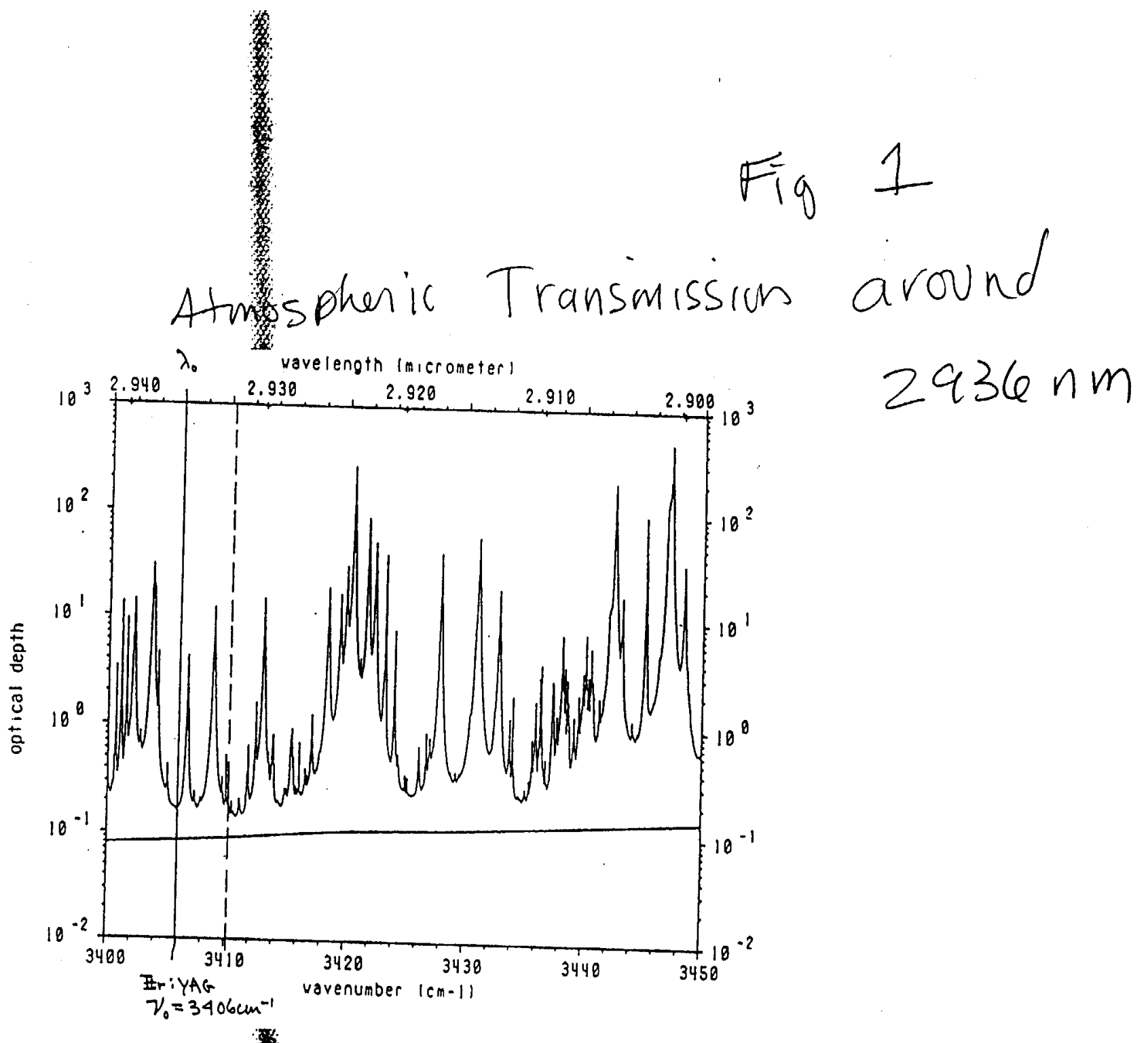




\section{Tunable, Diode-Side-Pumped Er:YAG Laser}

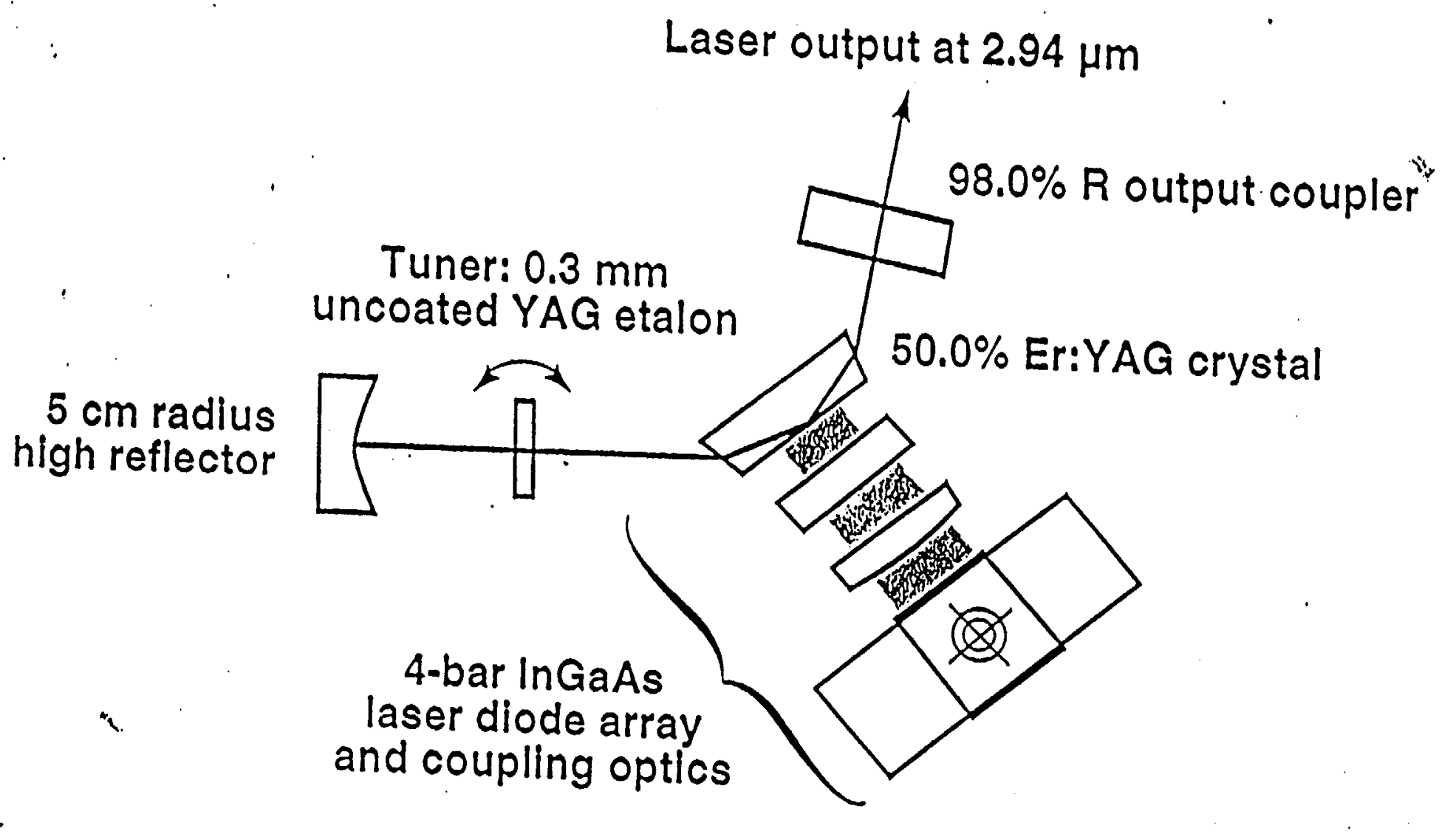




\section{Fig 3}

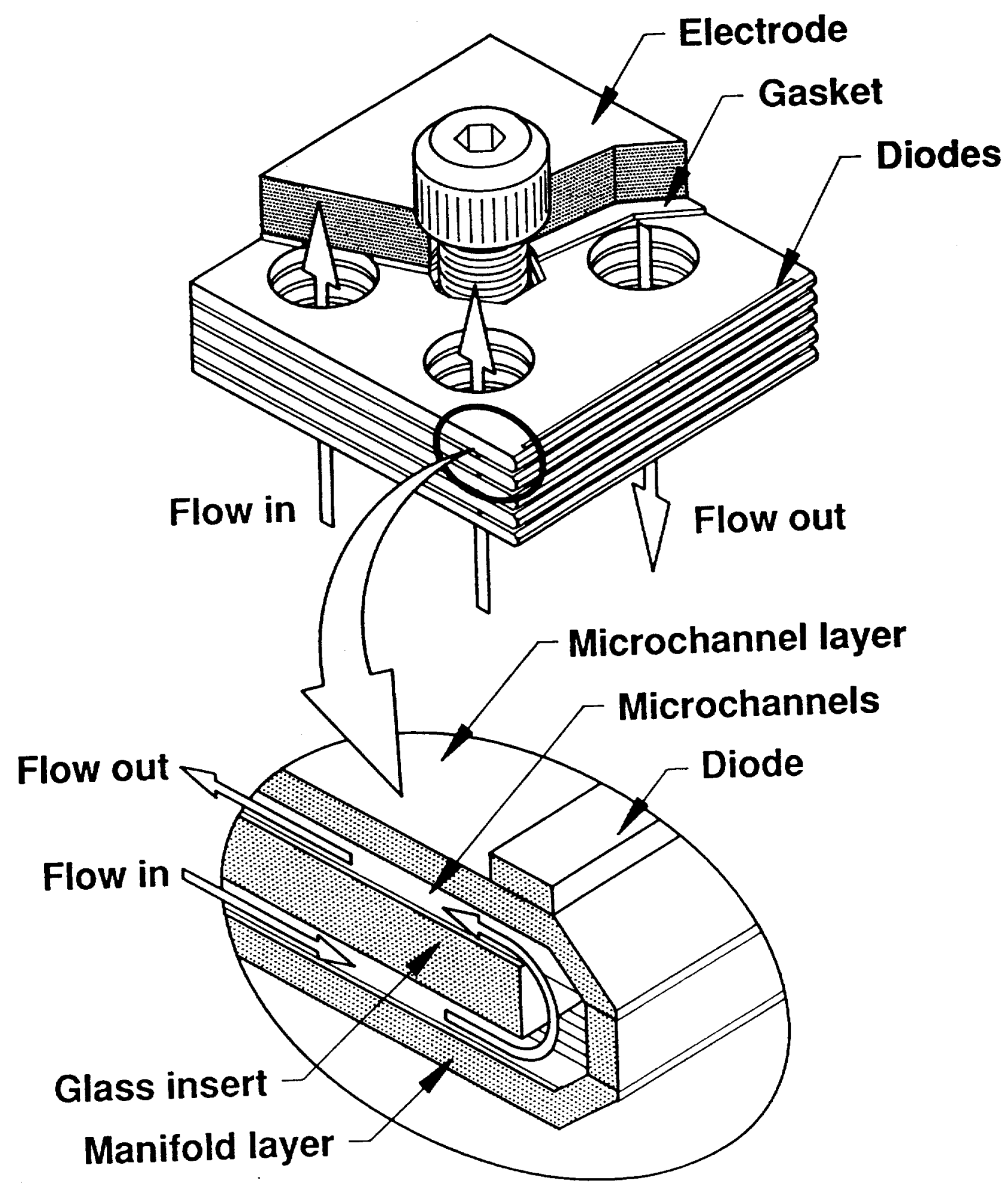



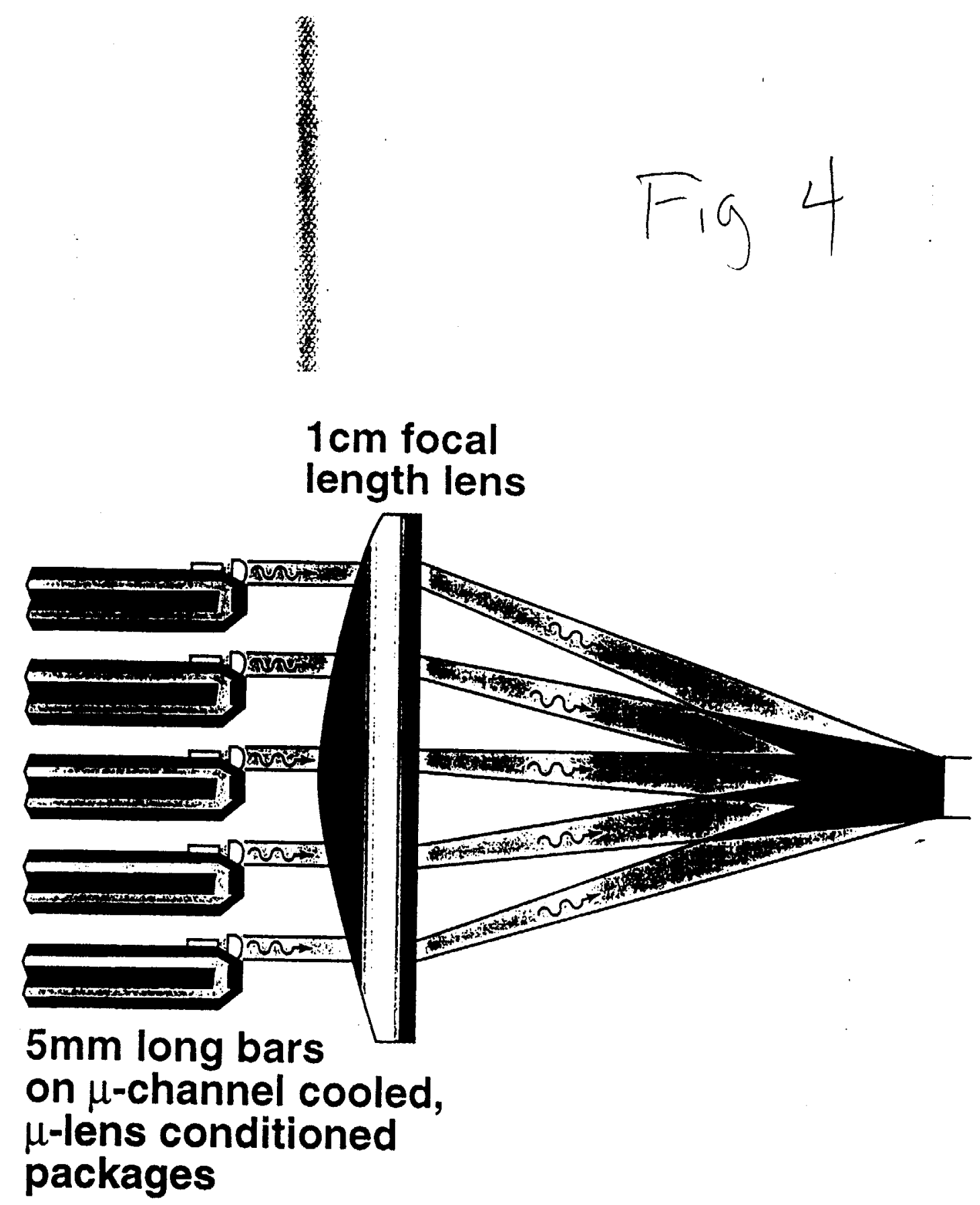


\section{$K H B$ \\ 50\% Doped Er:YAG \\ Fig. 5 \\ FEB ? ? 1996}

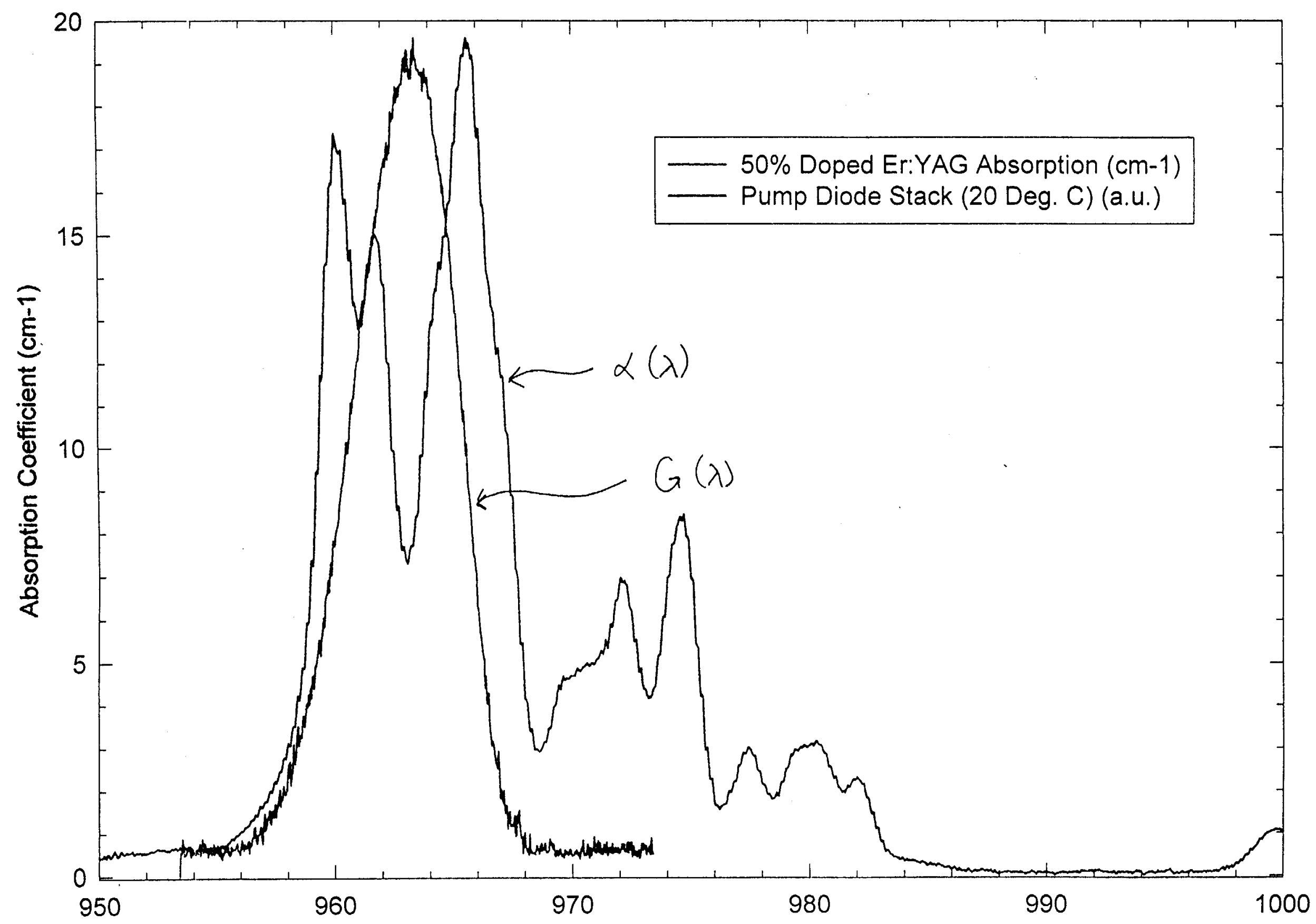




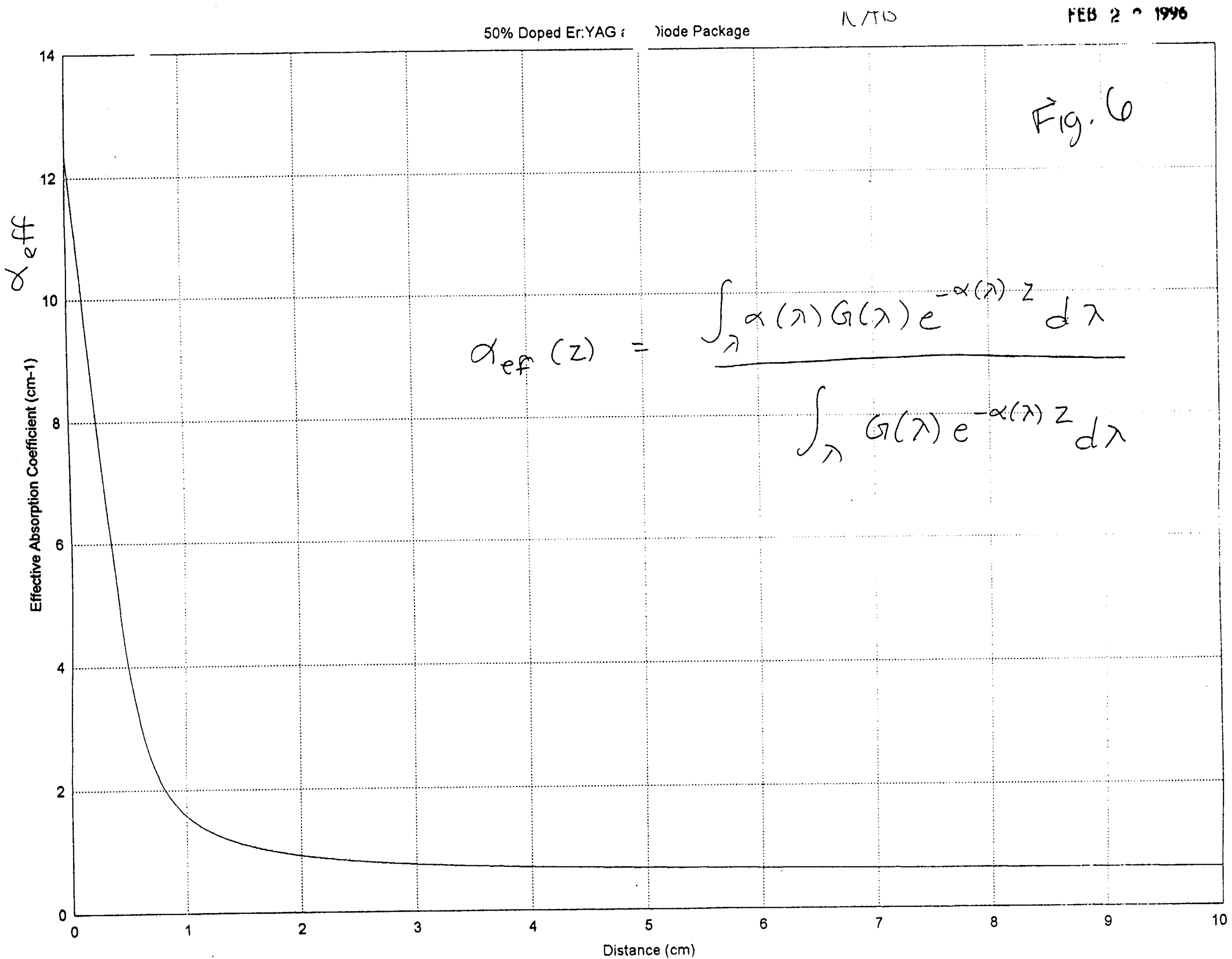




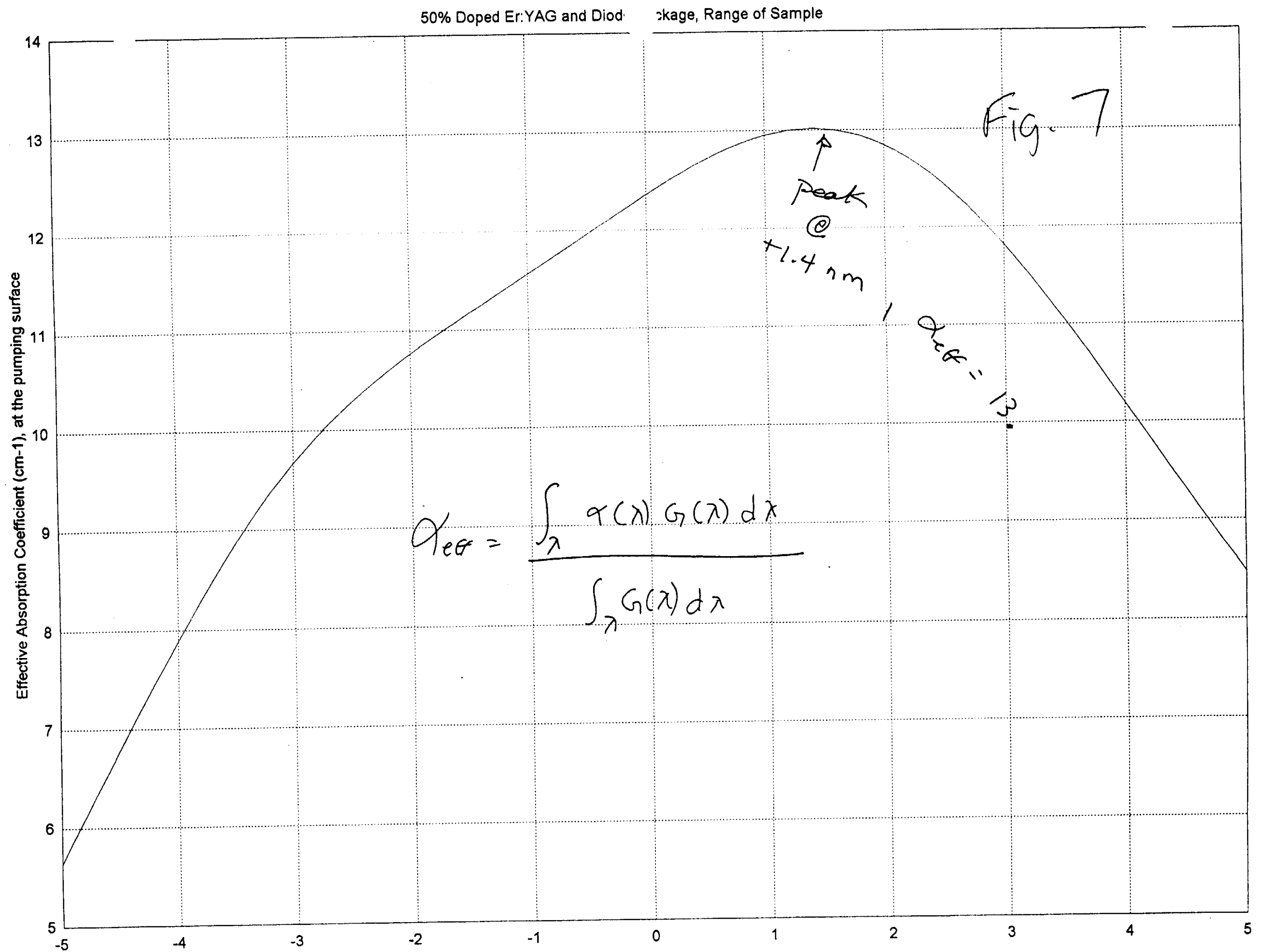


etrical detector (Laser Precision RJ 7100 ) ave ctrical detector (Laser Precision RJ 7100) averaging out a hundred laser pulses. The result is shown in Fig. :xpected, the lower the temperature the higher the emission energy and the lower the laser threshold. rabolic shape of $E_{\text {out }}$ against $E_{\text {in }}$ is believed to be due

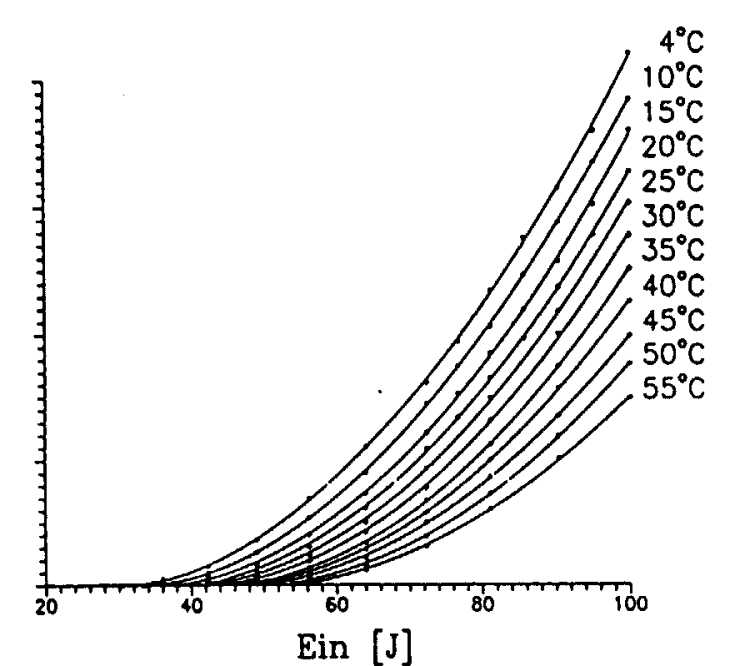

Laser pulse energy as a function of the electrical pump energy for iperatures of the crystal between 4 and $55^{\circ} \mathrm{C}$ at an average inpu $250 \mathrm{~W}$.

J. Appl. Phys., Vol. 69, No. 2, 15 January 1991 between the temperature dependent inversion $\left[N\left(X_{2}, T_{1}\right)-N\left(Y_{7}, T_{i}\right)\right]$ and the threshold energy

$E_{\mathrm{th}}\left(T_{i}\right)$, where $T_{i}$ is the temperature,
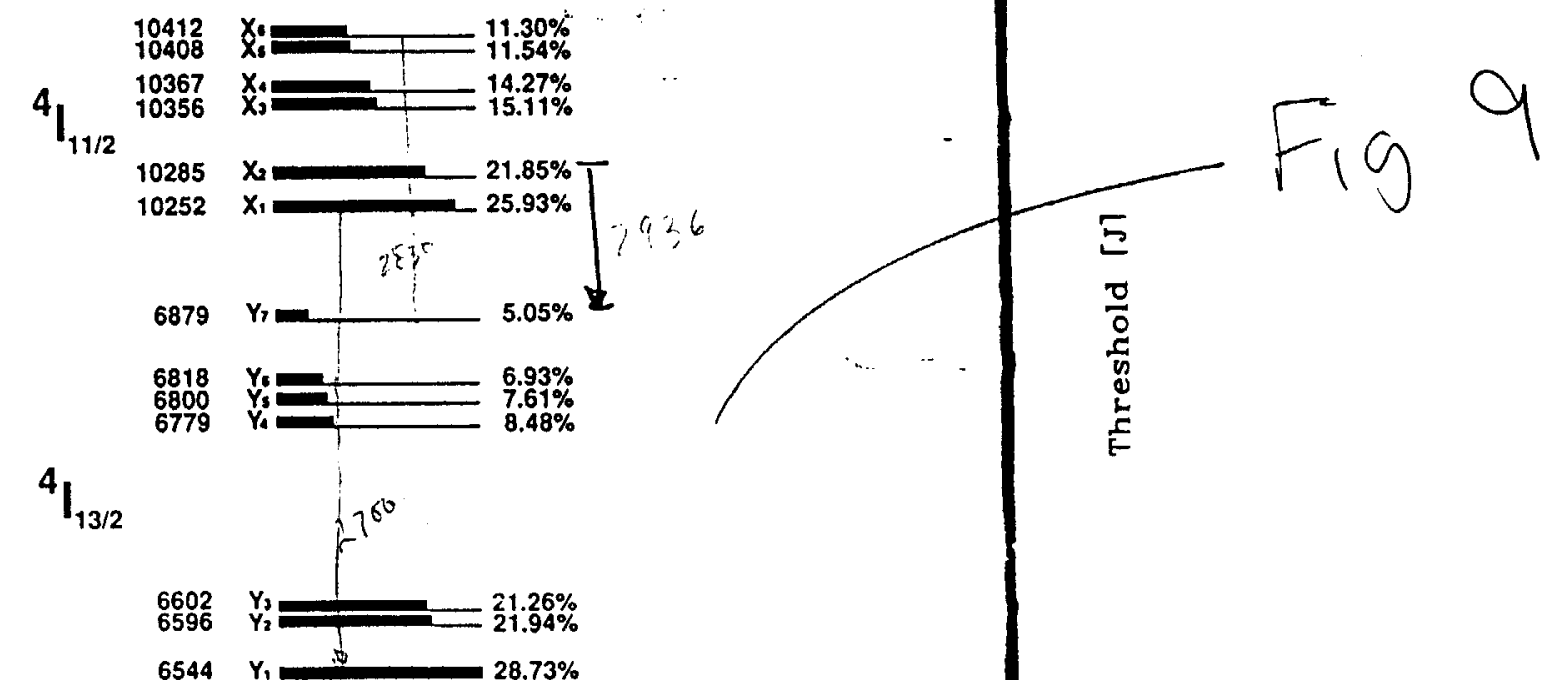

FIG. 4. The ${ }^{4} I_{11 / 2}$ and ${ }^{4} I_{13 / 2}$ manifolds of Er ${ }^{2}+$ ions in YAG. The energy is given in $\mathrm{cm}^{-1}$. The bars show the relative population of the Stark levels given in 1 . 1 according 10 a Bolzmann distribution a $4^{\circ} \mathrm{C}$ given in percent on the righthand scale at a core temperature of $4^{\circ} \mathrm{C}$. Stimulated emission occurs between $X_{2}$ and $Y_{7}$.

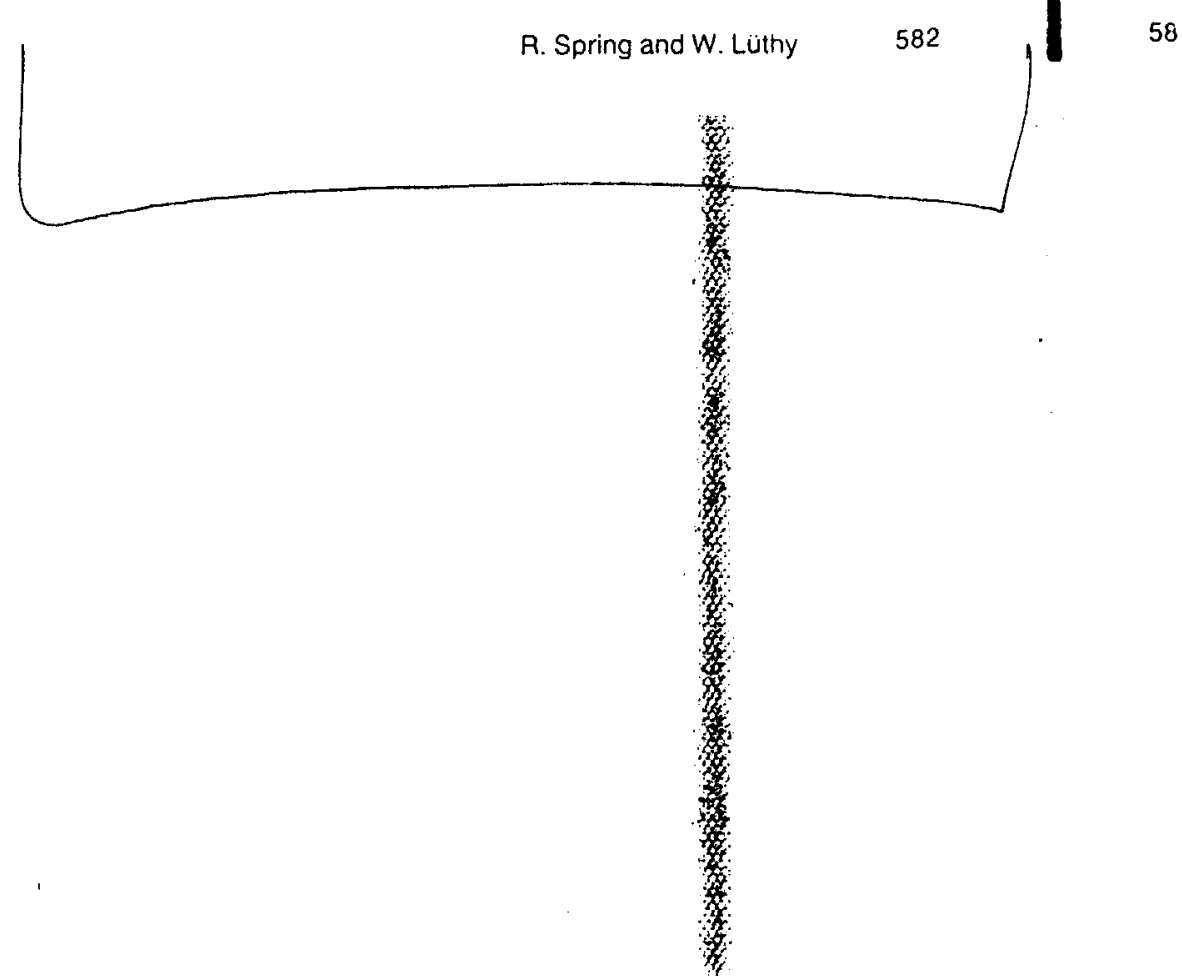




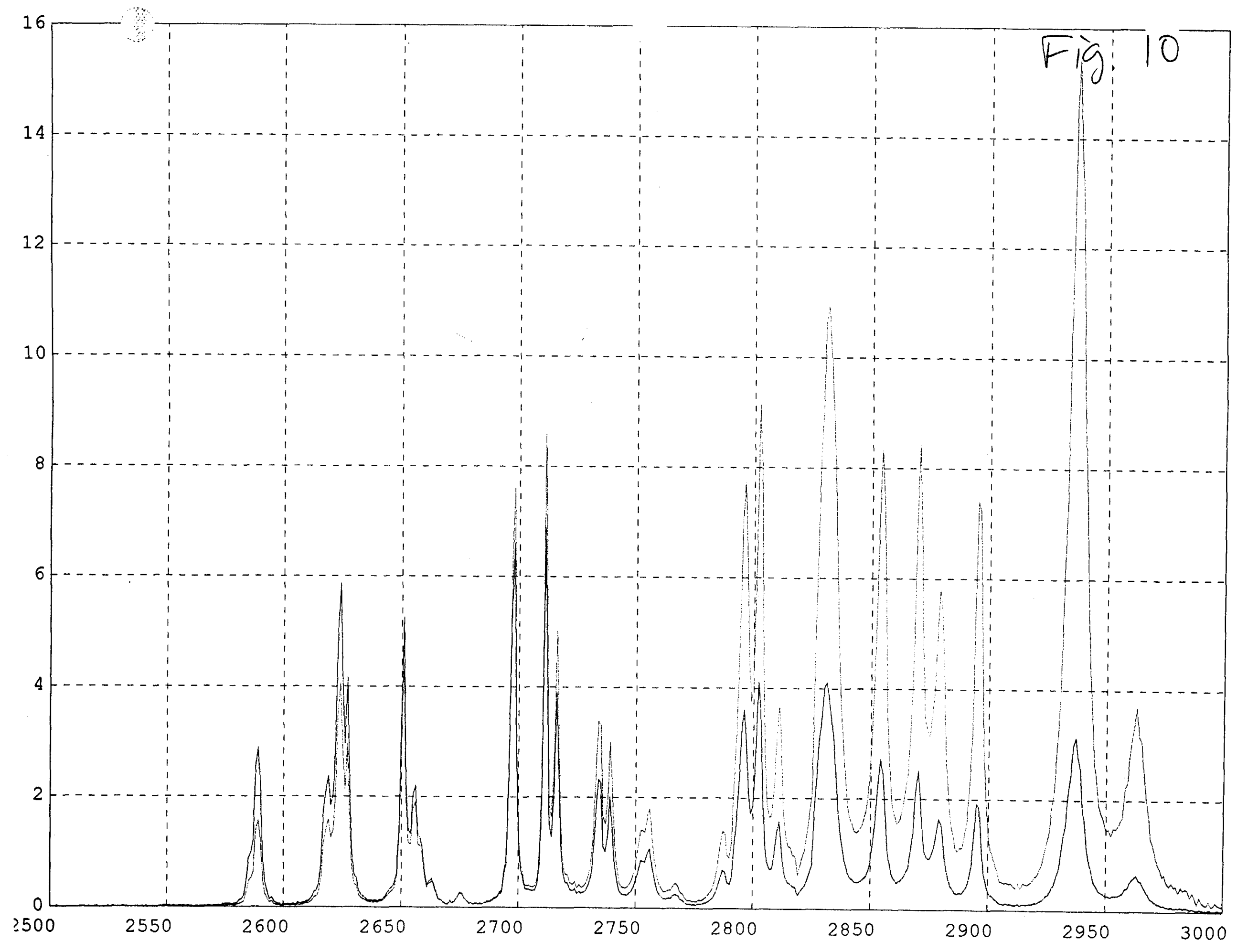




\section{Kinctios Model}

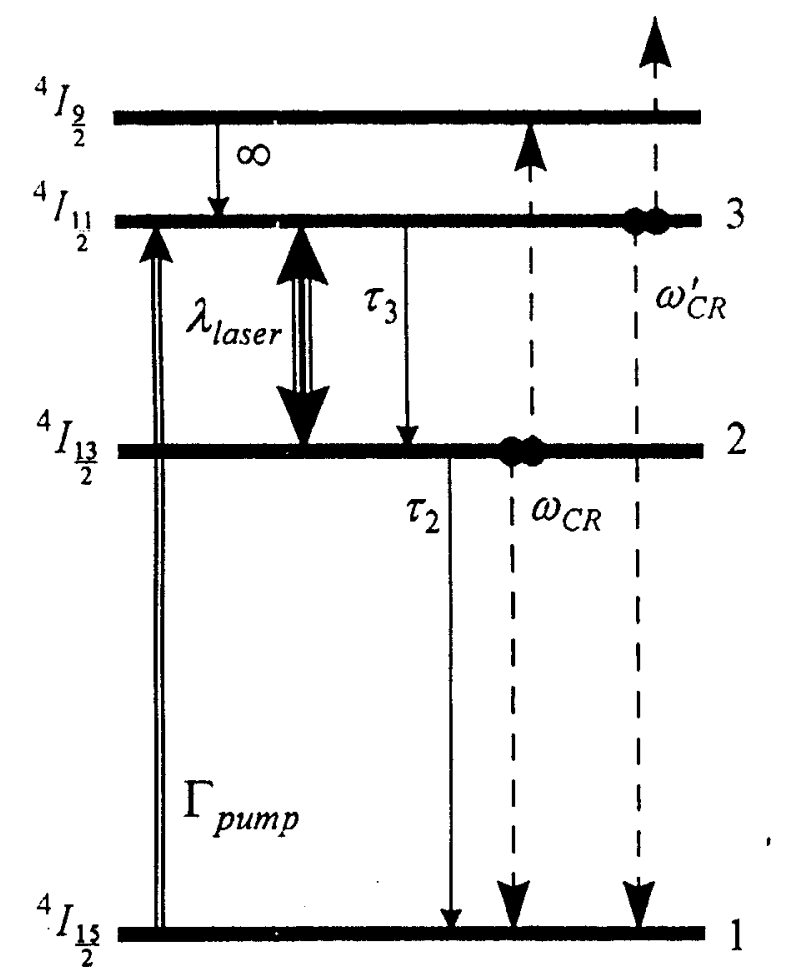

Track cavity photon number density in a number of discrete photon channels

$$
\begin{aligned}
& \frac{d \phi\left(v_{i}\right)}{d t}=\phi\left(v_{i}\right) \frac{l_{s} c}{l_{c}}\left[n_{3} \sigma_{e m s}\left(v_{i}\right)-n_{2} \sigma_{a b s}\left(v_{i}\right)\right]-\frac{\phi\left(v_{i}\right)}{\tau_{c}} \\
& \frac{d n_{3}}{d t}=\Gamma_{p u m p}-\frac{n_{3}}{\tau_{3}}+\omega_{C R} n_{2}^{2}-\omega_{C R}^{\prime} n_{3}^{2}-\sum_{i}^{n} \phi\left(v_{i}\right) \frac{l_{s} c}{l_{c}}\left[n_{3} \sigma_{e m s}\left(v_{i}\right)-n_{2} \sigma_{a b s}\left(v_{i}\right)\right]
\end{aligned}
$$$$
\frac{d n_{2}}{d t}=\frac{n_{3}}{\tau_{3}}-\frac{n_{2}}{\tau_{2}}-2 \omega_{C R} n_{2}^{2}+\sum_{i}^{n} \phi\left(v_{i}\right) \frac{l_{s} c}{l_{c}}\left[n_{3} \sigma_{e m s}\left(v_{i}\right)-n_{2} \sigma_{a b s}\left(v_{i}\right)\right]
$$

$$
\tau_{c}=-\frac{2 l_{c}}{c \ln \left(T^{2} R\right)}
$$

Ground State Depletion

\begin{tabular}{|c|c|}
\hline 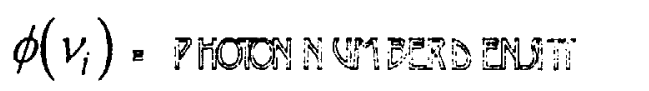 & $n_{E r^{3+}}=$ ERT A A D OPING D EUATI \\
\hline 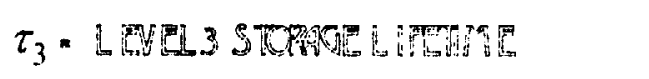 & $n_{3} \cdot$ LEVEL 3 D ERATT \\
\hline 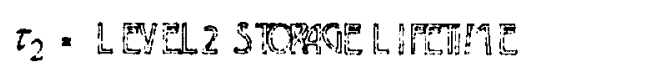 & $n_{2} \cdot$ \&EU $2 D$ DEMT \\
\hline 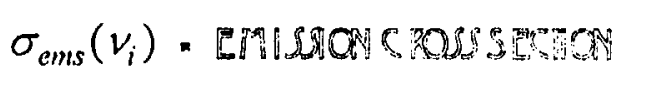 & 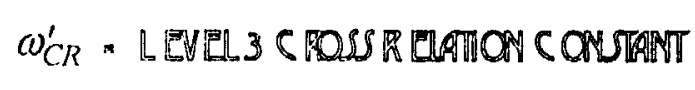 \\
\hline 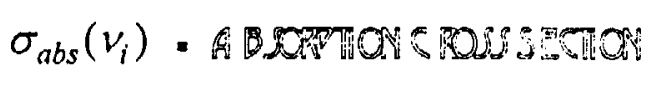 & 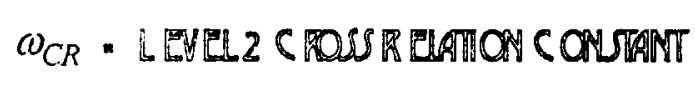 \\
\hline 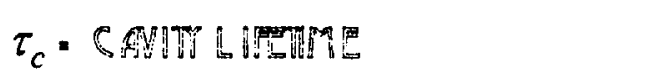 & $\Gamma_{\text {punp }}=$ PunP EXCIATON R RTL \\
\hline
\end{tabular}
fig.

$$
\Gamma_{p u m p}=\Gamma_{0}\left(1-\frac{n_{3}+n_{2}}{n_{E r^{3+}}}\right)
$$




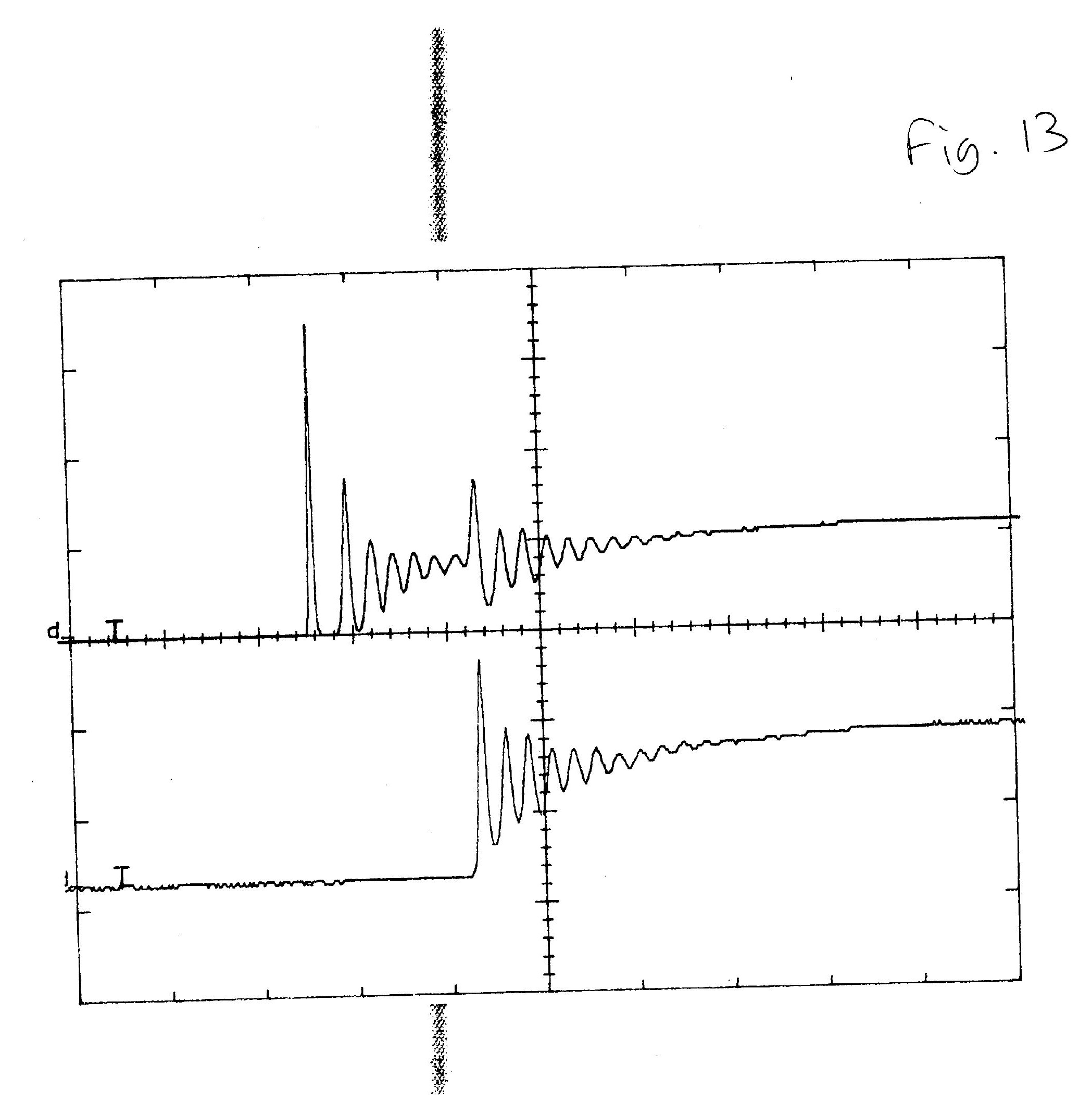




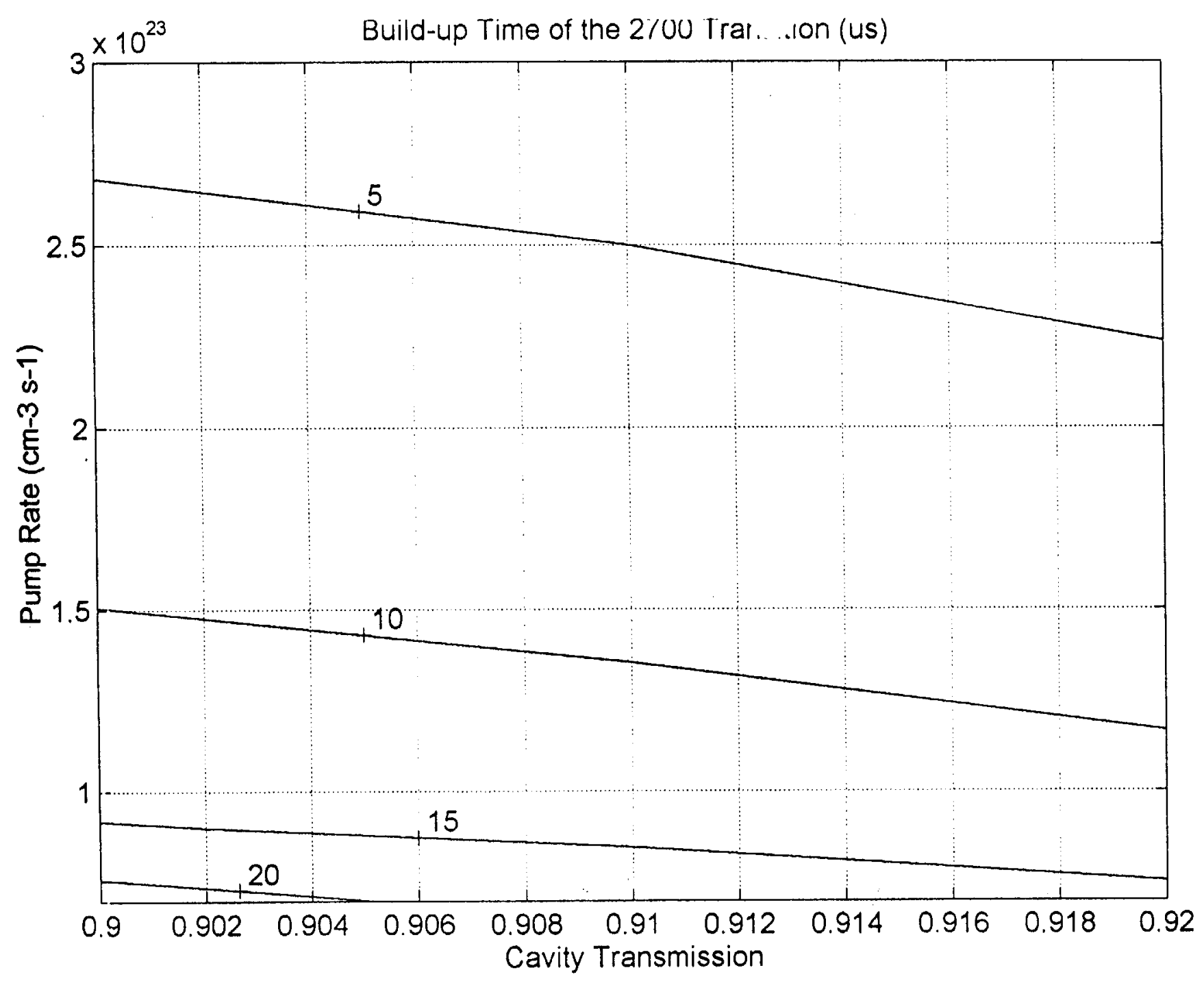

Fig. 14 


\section{MAR 281996}

Square Ended Slab, Straight Through center of rod

\begin{tabular}{|c|c|c|c|c|}
\hline Current $(A)$ & Power $(W)$ & Spot Size $(\mathrm{mm})$ & focal length $(\mathrm{cm})$ & inverse focal length \\
\hline 100.0000 & 13.0000 & 2.6200 & & \\
60.0000 & 7.5000 & 2.9200 & 8.1600 & 0.1225 \\
45.0000 & 5.4000 & 3.0900 & 12.0000 & 0.0833 \\
35.0000 & 4.0000 & 3.2900 & 14.9000 & 0.0671 \\
25.0000 & 2.5000 & 3.9600 & 36.5000 & 0.0274 \\
15.0000 & 1.1000 & 4.3000 & 90.0000 & 0.0111 \\
0.0000 & 0.0000 & 4.3000 & & 0.0000 \\
\hline
\end{tabular}
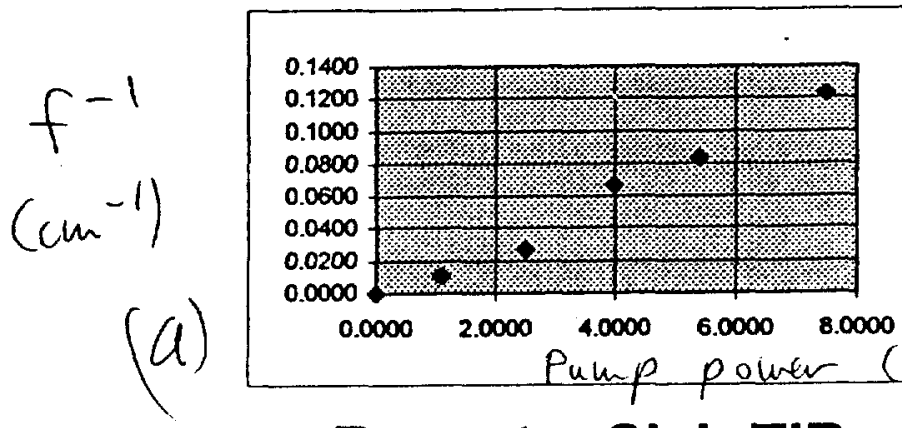

\section{Brewster Slab TIR}

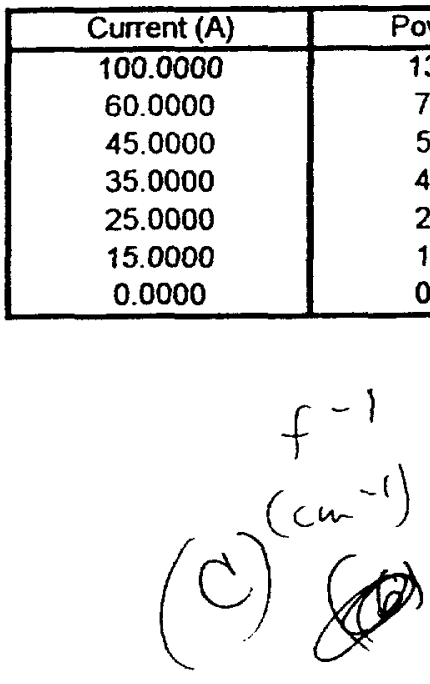

\begin{tabular}{|c|c|}
\hline ower $(\mathrm{W})$ & Spot Size $(\mathrm{mm})$ \\
\hline 13.0000 & 4.2000 \\
7.5000 & 2.7400 \\
5.4000 & 2.4600 \\
4.0000 & 2.6000 \\
2.5000 & 2.8600 \\
1.1000 & 3.1000 \\
0.0000 & 3.5260 \\
\hline
\end{tabular}

focal length Lum inverse focal length

$\left.\begin{array}{|c|c|}\hline 3.6000 & 0.2778 \\ 5.5000 & 0.1818 \\ 10.0000 & 0.1000 \\ 13.3000 & 0.0752 \\ 19.0000 & 0.0526 \\ 28.0000 & 0.0357 \\ & 0.0000\end{array}\right]$ Table 2

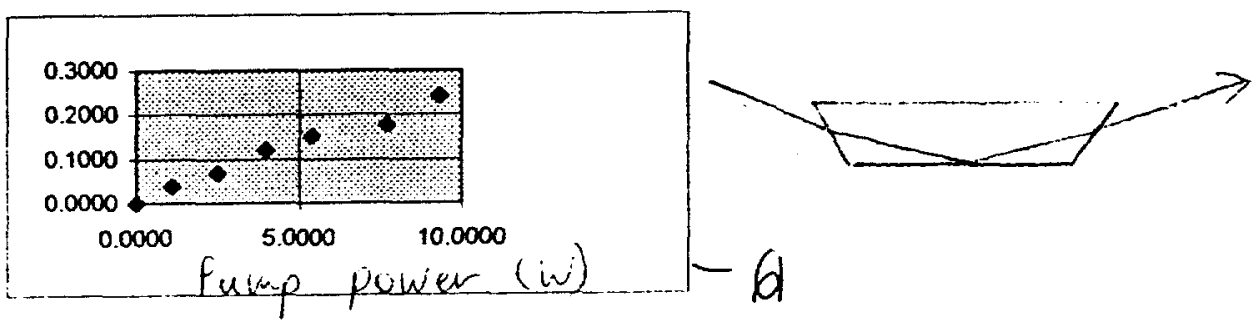

Square Ended TIR

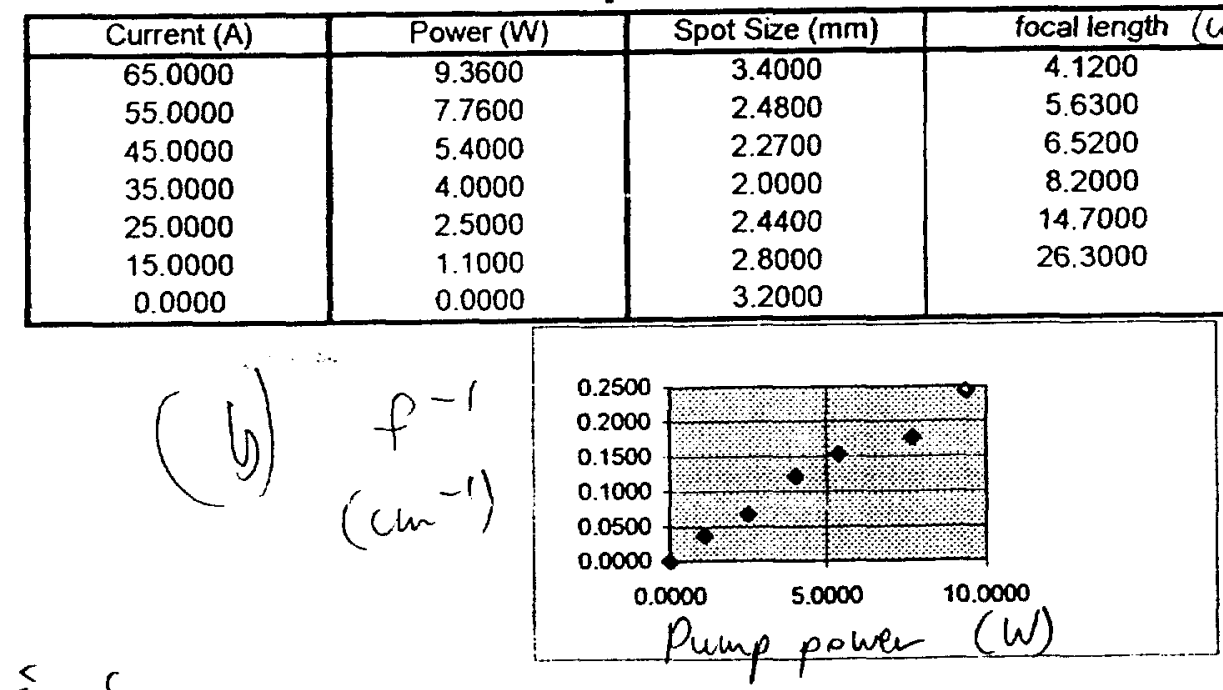

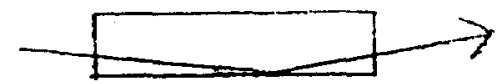




\section{Optical Properties}

Er:YAG - $\quad \frac{\partial n}{\partial \mathrm{T}}=8.9 \times 10^{-6} \mathrm{~K}^{-1}$

\section{Mechanical and Thermal Properties}

\begin{tabular}{|l|l|c|c|c|c|}
\hline \multicolumn{2}{|c|}{ Property } & \multicolumn{4}{c|}{ Material } \\
\hline Name & \multicolumn{1}{|c|}{ Unit } & Er:YAG & Sapphire & Copper & Indium \\
\hline \hline Thermal conductivity & $\mathrm{W} / \mathrm{m}-\mathrm{K}$ & 5.0 & 28.0 & 83.0 & 83.0 \\
& $\mathrm{~W} / \mathrm{mm}-\mathrm{K}$ & 0.005 & 0.028 & 0.083 & 0.083 \\
\hline $\begin{array}{l}\text { Thermal expansion } \\
\text { coefficient }\end{array}$ & $\mathrm{K}^{-1}$ & $67 \times 10^{-7}$ & $67 \times 10^{-7}$ & $9.2 \times 10^{-6}$ & $9.2 \times 10^{-6}$ \\
\hline Elastic modulus & $\mathrm{Pa}$ & $282 \times 10^{9}$ & $405 \times 10^{9}$ & $117 \times 10^{9}$ & $117 \times 10^{9}$ \\
& $\mathrm{~N} / \mathrm{mm}^{2}$ & $282 \times 10^{3}$ & $405 \times 10^{3}$ & $117 \times 10^{3}$ & $117 \times 10^{3}$ \\
\hline Poisson's ratio & & 0.28 & 0.25 & 0.4 & 0.4 \\
\hline
\end{tabular}

Table 3 


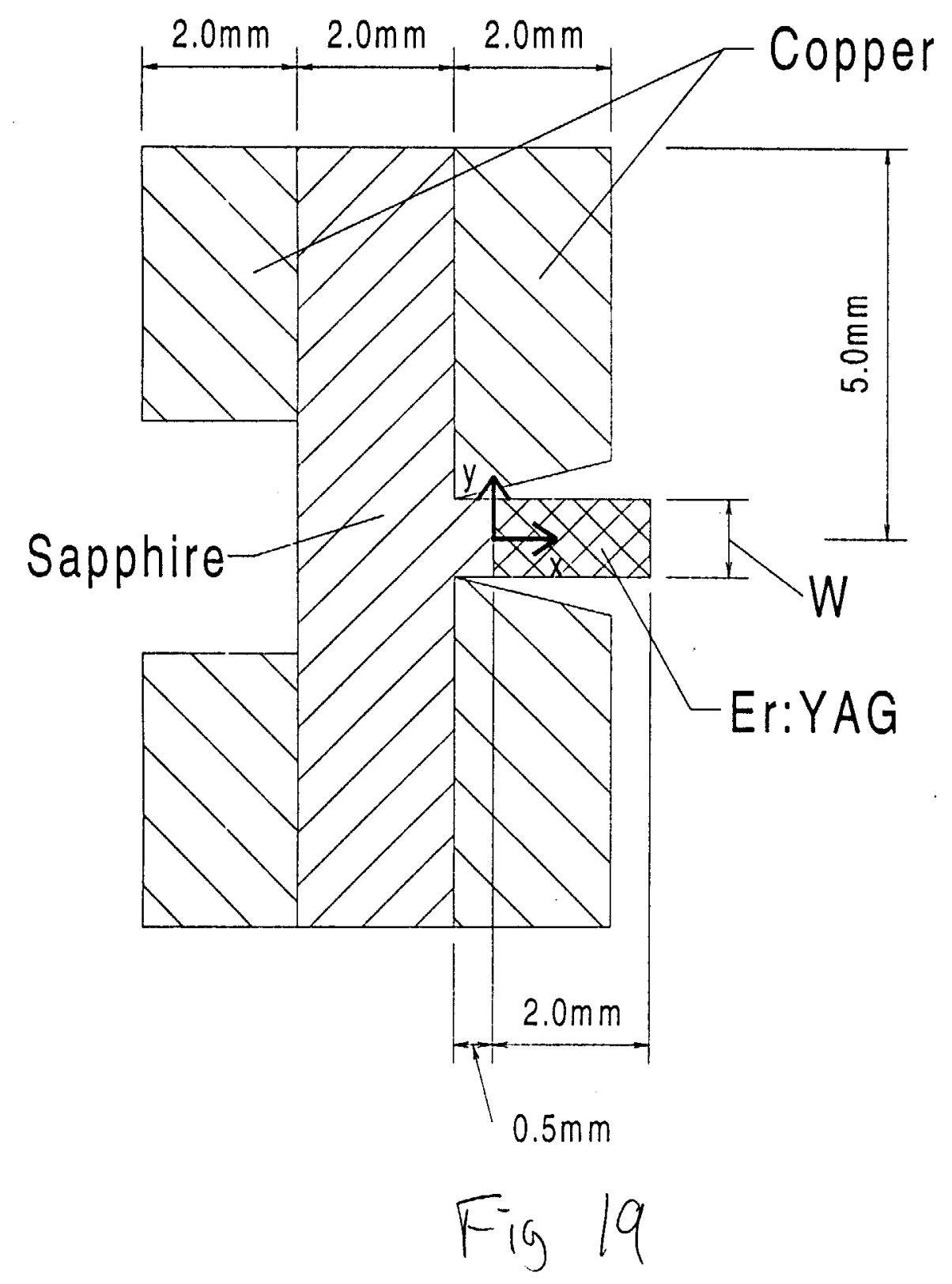

Geometry \#1

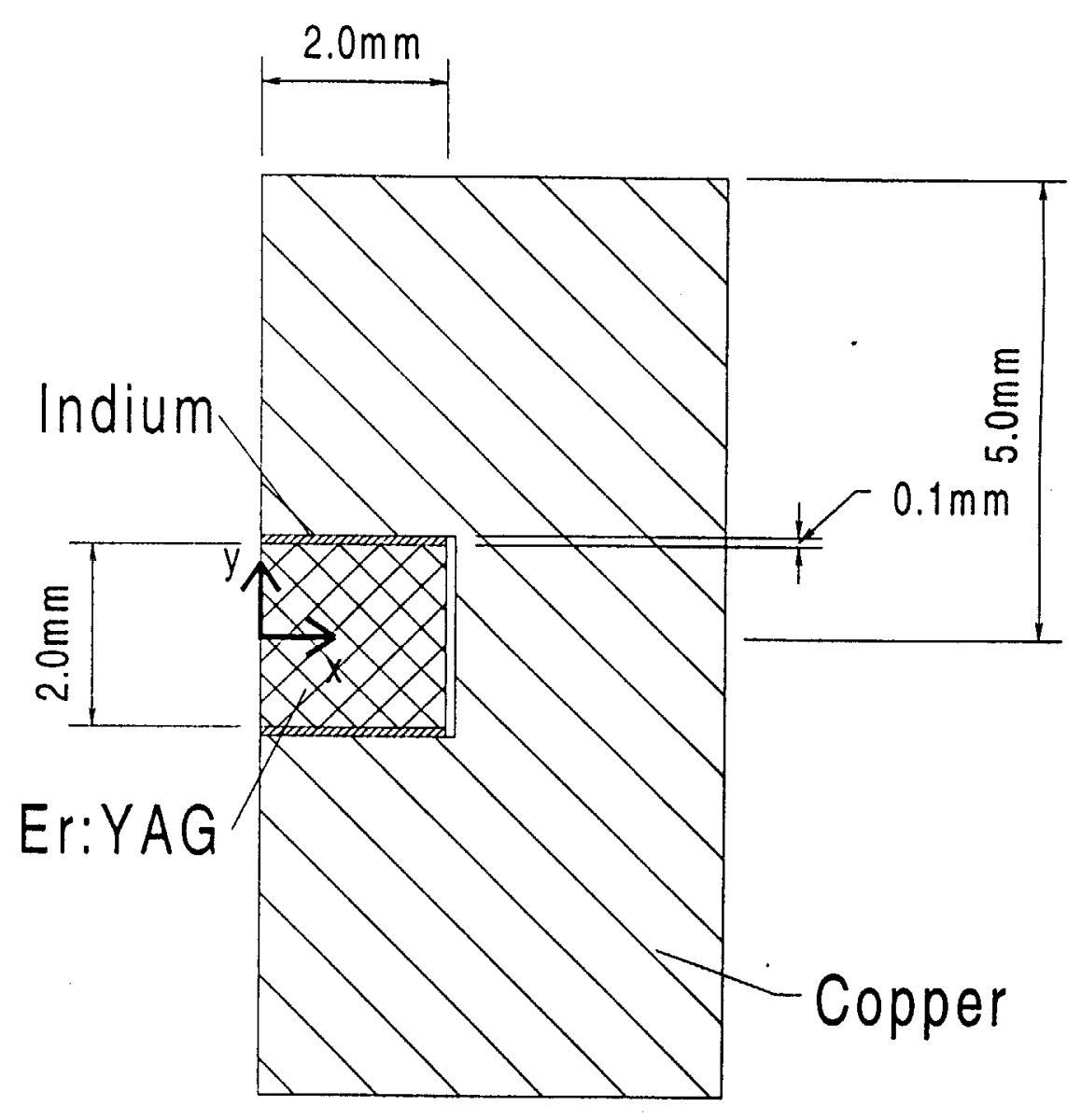

$$
F \log 10
$$

Geometry \#2 


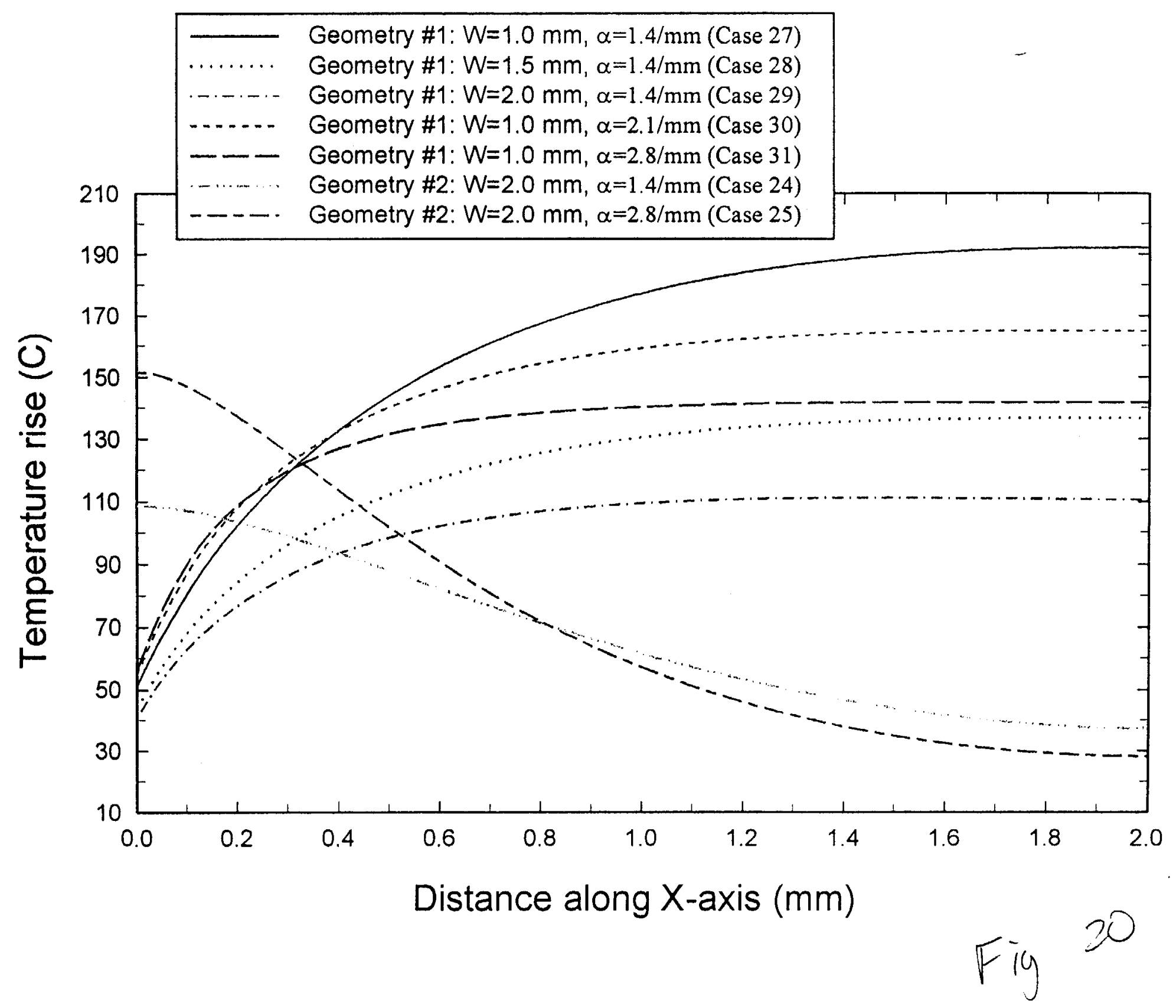




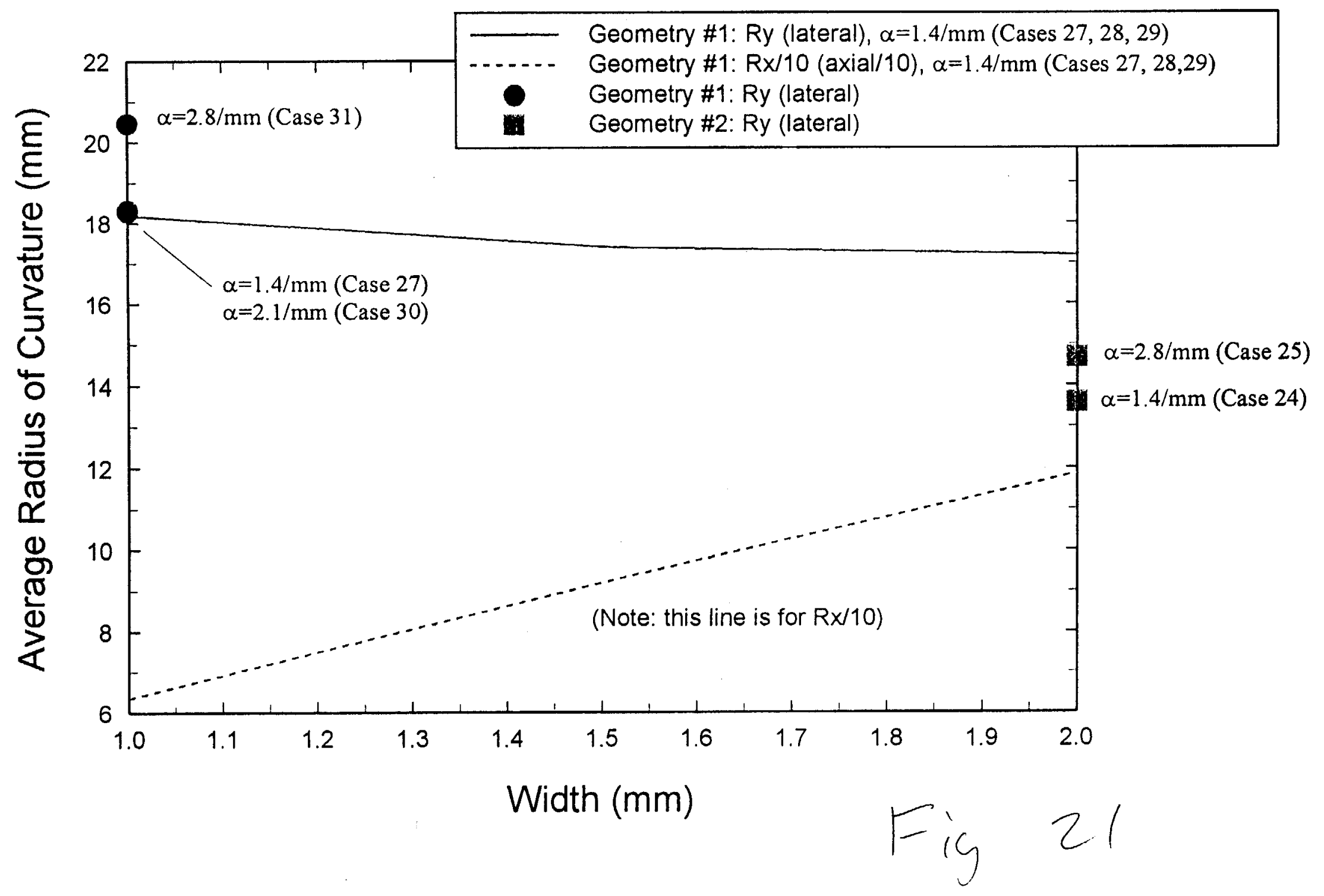




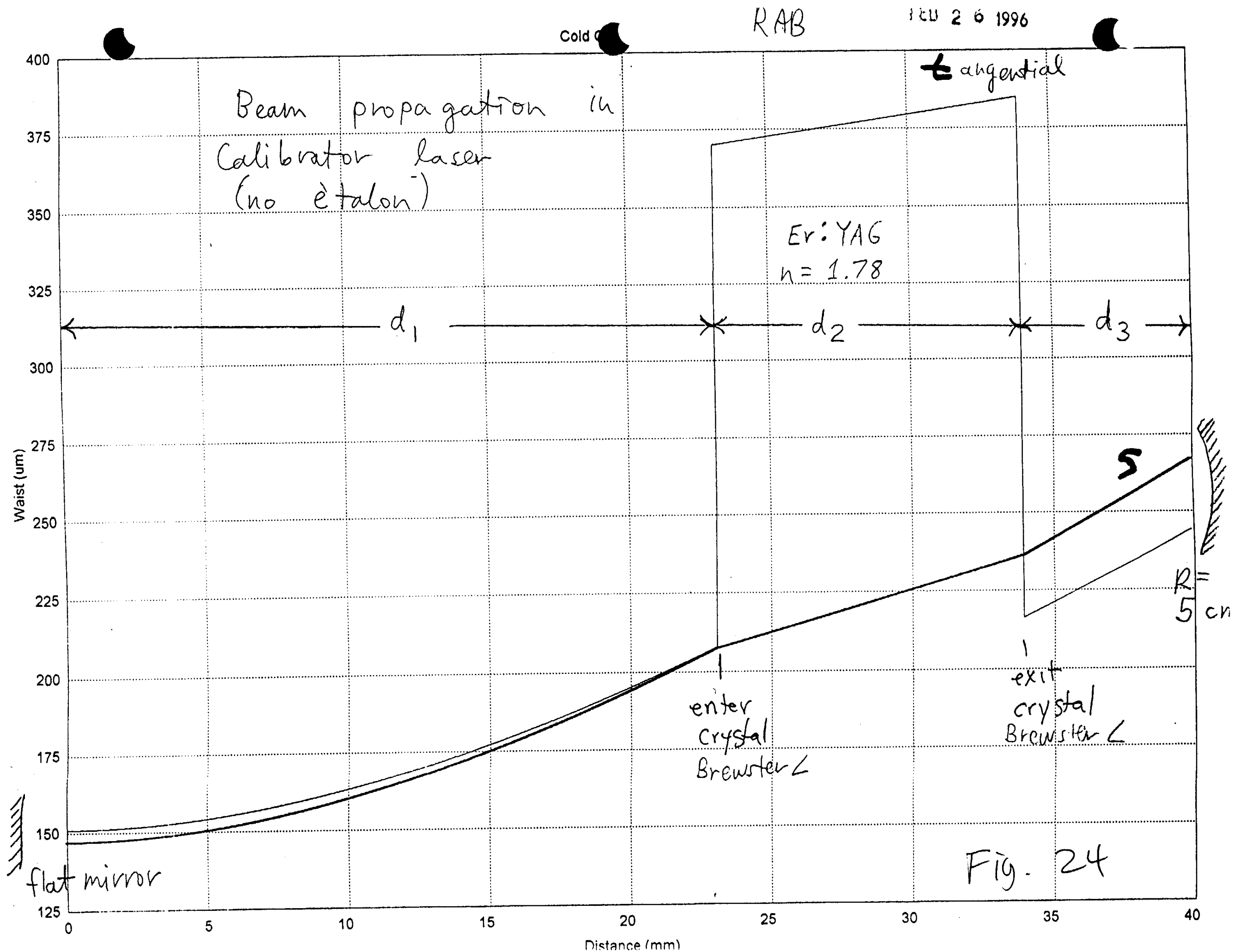




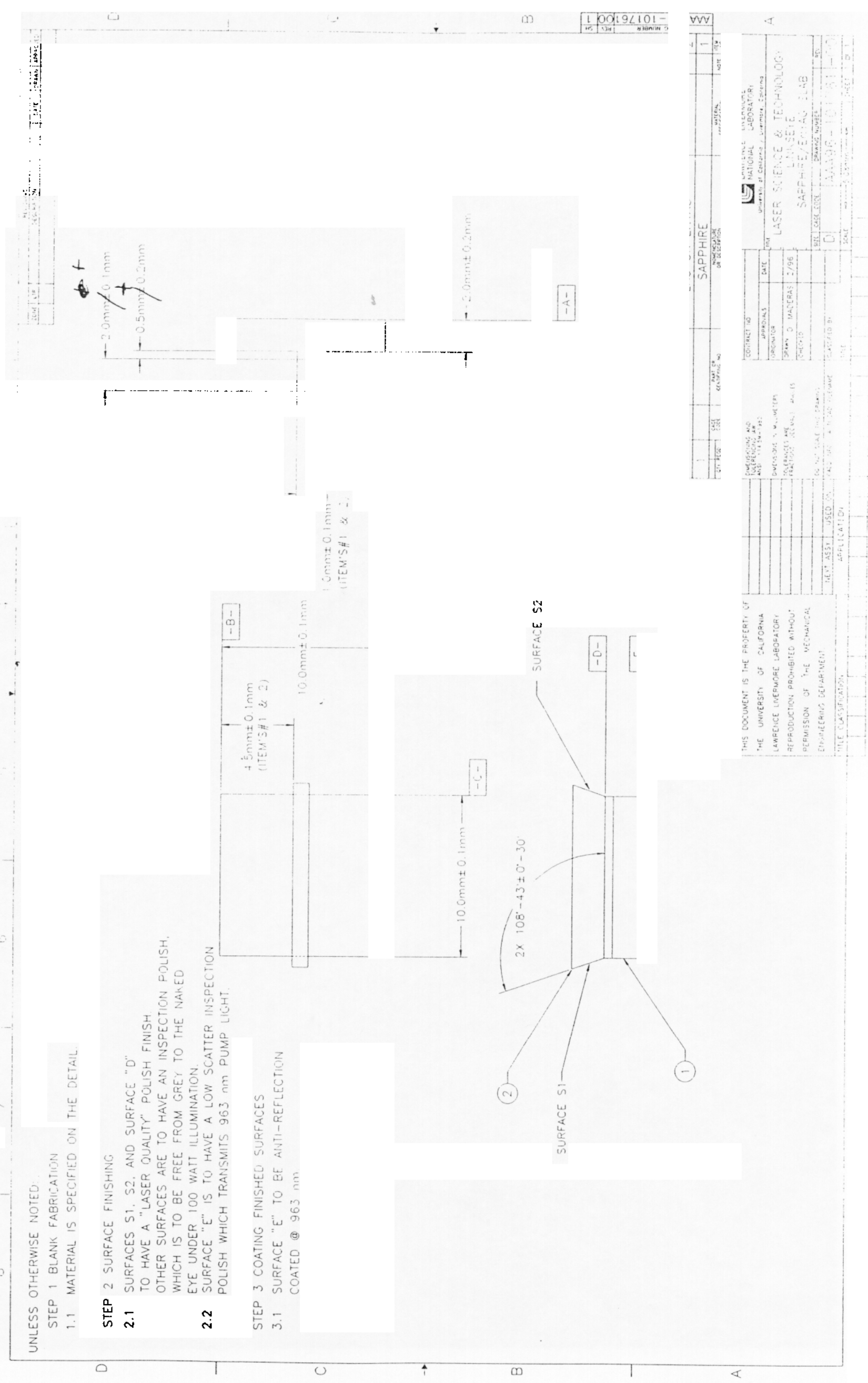



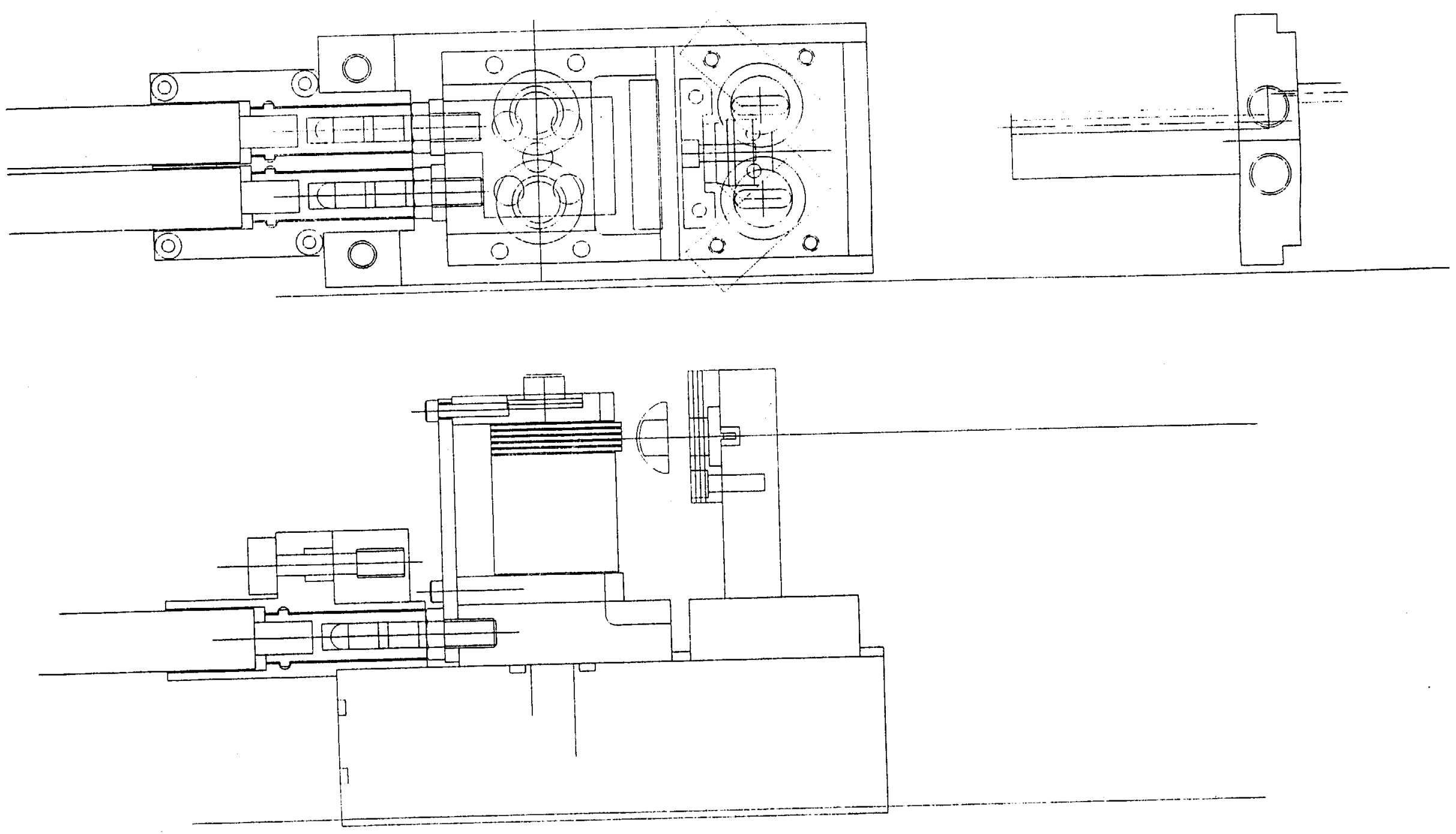


\title{
LLNL'S INTERNAL DISCHARGE LIMITS REVISION
}

\author{
Katherine deRopp \\ University of Tennessee
}

\section{Lawrence Livermore National Laboratory \\ Livermore, CA 94550}

May 8, 1996

Prepare in partial fulfillment of the requirements of the Science and Engineering Research Semester under the direction of Robert Vellinger, Research Mentor, in the Lawrence Livermore National Laboratory.

* This research was supported in part by an appointment ot the U.S. Department of Energy Science and Engineering Research Semester (hereinafter called SERS) program administered by LLNL under Contact W-7405-Eng-48 with Lawrence Livermore National Laboratory.

If this paper is to be published, a copyright disclaimer must also appear on the cover sheet as follows:

By acceptance of this article, the publisher or recipient acknowledges the U.S. Government's right to retain a non-exclusive, royaly-free license in and to any copyright covering this article. 


\title{
LLNL'S INTERNAL DISCHARGE LIMITS REVISION \\ Katherine deRopp
}

\begin{abstract}
:
\end{abstract}
This study identifies technically based internal discharge limits for LLNL's Wastewater Policy Document revision. To accurately develop LLNL's internal limits, I evaluated the different treatment processes at the Livermore Water Reclamation Plant (LWRP). I used PRELIM modeling software to calculate the LWRP's allowable headworks loading. The unallocated portion was then determined, applied to LLNL according to their flow, and compared with current discharge limits. I then reviewed the technical-basis of the current internal limits and evaluated each of LLNL's internal discharge sources. I calculated a combined mass loading for these sources, compared them with B196 calculated discharge loadings, and obtained a percent difference.

The net result is that LLNL is not using their available loading capacity for metals; and LWRP's treatment capabilities are underutilized. Potential economic savings are available from reduced wastewater handling and associated costs. An Excel model is being developed using LLNL's internal sources to calculate appropriate internal discharge guidelines for the sanitary sewer. The model will improve wastewater management of process discharges and calculate their loading impact on the LWRP. 


\section{INTRODUCTION:}

Lawrence Livermore National Laboratory (LLNL) is currently in the process of revising their Wastewater Discharge Guidance Document; therefore, this is the best time to review current guidance and practices. This document lists the internal discharge limits and gives appropriate guidelines for discharges to the sanitary sewer. Since the last revision six years ago, regulations and applicable regulatory limits have changed; as well as the processes that discharge to the sanitary sewer. The wastewater from buildings within LLNL with the potential to generate pollutants in significant quantites is retained and analyzed before being released to the sanitary sewer system. A Retention System Disposition Record (RSDR), which lists the results of the analysis, must be reviewed before the tank's release. Discharges into the sewer from waste retention tanks must comply with all regulations and meet the internal discharge limits. The Water Guidance and Monitoring Group (WGMG) develops the internal limits for the most common and heavily regulated wastewater pollutants to comply with the B196 discharge limits and protect the LWRP's treatment processes. Therefore, I embraced a project to develop technically based internal limits that are protective. I have evaluated the technical basis of the current B196 outfall and internal discharge limits, and whether to incorporate a mass loading into the limit's revision. Also, the possible cost savings that may be gained by revising LLNL's internal discharge limits.

All sewered water from the Sandia Laboratory and LLNL is monitored at the northwestern corner of the lab at building 196; the 
effluent outfall point of compliance for LLNL. Flow and $\mathrm{pH}$ are continuously monitored and recorded. Flow-proportional composite samples and grab samples are collected and analyzed for levels of metals, radioactivity, organics, and water quality parameters. The levels of these contaminants are summarized in a monthly report (Table 1). A 1 gpm sewage stream is continously diverted into the online monitoring station located inside B196. The sample then flows through a series of four flow cells where it is analzed for levels of radioactivity and the nine regulated metals. If a contaminant is present above a predetermined alarm value, a computer initiates a diversion of flow to holding tanks to protect the public from any harmful levels of pollutants that could be released into the sewer system. This monitoring also protects the LWRP from any harmful spills that might inhibit their treatment processes.

\section{PROIECT FINDINGS:}

Regulating a mass loading at B196 or internally is the most effective way to manage wastewater because both the concentration and discharge flowrate are taken into consideration. A pollutant mass from each internal source was calculated using the discharge volume and concentration. The internal sources that discharge to B196 include the influent water supply from Hetch-hetchy reservior, Sandia Lab discharges, HWM/retention tanks, cooling towers, berm water, and mop water. I calculated a mass loading using the following equation:

Mass loading $(\mathrm{lb} / \mathrm{d})=[$ conc. $(\mathrm{mg} / \mathrm{l}) *$ flowrate $(\mathrm{Mgd}) * 8.34(\mathrm{lb} / \mathrm{Mg} * \mathrm{mg} / \mathrm{l})]$ 
To perform a mass-balance comparison between the known internal sources and what was known to be discharged at B196, I then;

- Calculated and summed a mass loading for each influent source.

- Calculated a mass loading for the effluent discharge point.

- Calculated a percent difference between these two totals (Table 2).

This mass-balance anaylsis enables LLNL to understand the amount of pollutant loading contributed by each internal discharge source and compare it to the B196 effluent loading. For six of the pollutants analyzed, the effluent is higher than the influent; thus not all of the discharge sources have been identified. Silver processors located around the lab are being anaylzed as a possible influent contributor. A better understanding of the influent discharges to the sanitary sewer will decrease the percent difference. The objective is to obtain a percent difference of $20 \%$ or less for each pollutant in order to correctly model the wastewater stream.

To calculate technically based internal limits that will maintain compliance at the outfall point, the B196 permit limits must not be exceeded; or LLNL risks a notice of violation (NOV) from the LWRP. Therefore, I wanted to evaluate whether the permit limits, set by the LWRP, are too conservative. This requires a full understanding of the operations at the LWRP. The LWRP has different treatment processes that remove pollutants from wastewater. Different percent removal efficiencies exist for each pollutant parameter. 
For example, to calculate the percent removal efficiency for copper:

$$
\% \text { removal efficiency }=\frac{\text { influent loading }- \text { effluent loading }}{\text { influent loading }} * 100 \%
$$

$\%$ removal efficiency $=2.45(\mathrm{lbs} / \mathrm{day})-31(\mathrm{lbs} / \mathrm{day}) * 100 \%$

2.45 (lbs/day)

Copper removal efficiency $=87.3 \%$

The influent to the LWRP first goes through a primary process which allows the sewage to settle so that the larger particles sink and can be scraped off the bottom as sludge (Figure 1). The secondary treatment process involves adding oxygen to the wastewater to allow microrganisms to feed and sink to the bottom as activated sludge (Figure 2). Then the sewage is allowed to settle again in the secondary clarifier. The effluent is chlorinated and then discharged to the San Francisco Bay via the LAVMA and EBDA pipelines. Some of the wastewater goes through a tertiary process which uses chlorine and a polishing sand filter to cleanse the waste stream so that it can be used as reclaimed water for irrigation of a nearby golf course, airport, and highway.

The LWRP uses a PRELIM model to calculate the limits that restrict industry's outfall pollutant concentrations (B196 limits). PRELIM also performs a mass-balance check and determines the maximum allowable headworks loading (MAHL) in pounds/day for the 
facility by selecting the the most limiting treatment process (passthrough, inhibition, or sludge) for each pollutant.

Based on the PRELIM model results for copper, almost fifty percent of the LWRP's MAHL is unallocated to either industrial or domestic (Figure 3). Domestic loading contributions of copper are ten times greater than all industrial loadings combined. LLNL represents $71 \%$ of the industrial slice for copper. The twenty percent safety factor allows for future growth, domestic and/or industrial, that may occur in the next several years. If LLNL discharged at the maximum flow and concentration limits, as set by the LWRP, the loading would equal 14.07 (lbs./day). This would exceed the MAHL of the LWRP by 10.11 (lbs./day). Therefore the current limits are not protective of the LWRP's treatment operations for copper.

According to Figure 4, the loading that could be available to LLNL, apportioned by our flow and what the LWRP can accept, is much greater than what we are actually discharging. This leads to the conclusion that existing internal discharge limits are too restrictive and extra capacity is available from LWRP's treatment processes. The LWRP is currently recalculating revised limits which could affect the loading available to LLNL.

The City of Livermore's (LWRP) limits are the most restrictive of the six treatment facilities or Public Owned Treatment Works (POTWs) that discharge to the East Bay Discharge Authority (EBDA) pipeline (Table 3). This directly affects the process in which LLNL's internal limits are calculated and available sewer capacity. One reason for this conservative approach of the LWRP is that LLNL is the only major industry that has the potential to discharge elevated levels of 
pollutants into the waste stream. Since the last time the LWRP revised their limits, LLNL has gone through some major changes. Pretreatment regulations have changed, process discharges have changed, and the flow stream is decreasing. Therefore, the conservative approach may no longer be necessary. LWRP staff agrees that their facility is underutilized and they have available capacity. The treatment processes will operate more efficiently with a greater influent loading at the headworks. In fact, it would be safer to discharge a greater amount of nonhazardous pollutants to a POTW with removal capabilites than to handle, transport and potentially generate wastes to be disposed of a hazardous somewhere else.

\section{PROJECT RESULTS:}

The economic savings that could result from the revision of the internal limits are an important incentive. More managed discharges to the sanitary sewer will decrease the analytical, shipping, and handling costs. Higher discharge limits would reduce the treatment costs since many wastewater batches may go through several rounds of treatment before meeting the internal discharge limits. Reducing the amount of treats a batch of waste must go through directly results in a reduction in the volume of legacy waste generated from Door Oliver cuttings. The handling and sampling of this legacy waste is extremely expensive. Less managing of the berm water, which is mainly rainwater, could also result in significant cost savings. Developing and implementing such revised limits requires close cooperation with LLNL's Hazardous Waste Management (HWM) 
Division, and will result in better wastewater management; therefore saving LLNL money.

I calculated a set of revised internal discharge limits to be implemented in three phases (Table 4). In phase 1, the internal limits can reach but not exceed hazardous waste levels. Approval from EPD management has been obtained to proceed with development and implementation of this phase. In phase 1, administrative wastewater controls will be re-engineered. A new model, specific to LLNL's discharges, is in preparation to evaluate retention tank discharges. The RSDR process will need to be re-engineered with the new internal limits to incorporate an appropriate discharge volume and time of release. In phase 2, the Domestic Sewage Exclusion (DSE) as implemented by the CAL EPA (DTSC) in California must be fully explored. Some interpretations of the DSE would allow discharges above hazardous waste internally, as long as the B196 limits were still be met. LLNL plans to become involved in the DTSC regulatory process to allow the use of the DSE in CA. In phase 3, the LWRP would allow LLNL to raise the B196 compliance concentration limits or change to a mass-based compliance. In this phase, the cost/benefits of mass-based compliance will have to be further evaluated as well as plans for implementation. The model, once complete, would calculate the pollutant mass according to the selected phase.

This model, still in its formative stages, allows constant monitoring of tank discharges and their subsequent impact on the B196 limits and the LWRP. Once the model is complete, the wastewater can be managed either on a mass or concentration basis. Discharge authorities could permit certain volumes to be discharged at 
a specified flowrate during a specified time to assure compliance at the B196 outfall. The model will allow a better understanding of sewer capacity and also assist with spill traceback capabilities. If the LWRP allows compliance monitoring on a mass-based criteria, the model will be able to quickly calculate the allowable mass for that day. The results of such a model might also provide a more detailed understanding of dilution and dispersion which would occur after release of a tank. Another benefit might result in identification of additional influent sources and effects of non-tank related discharges to the sewer systemn. Pilot testing with HWM will be helpful in reflecting actual waste loading profiles; and with checking the model's validity as it is developed. Once the model has been completed, LLNL will have a better understanding of its internal discharges and Sandia Lab's contributions to our flow stream.

\section{SUMMARY:}

This project aimed at developing internal discharge limits with technical merit. Since LLNL's Wastwater Discharge Guidance Document is being revised, this is the time to establish technically-based limits. My approach was to review the present internal limits and current practices. I then evaluated the effects of relaxing administrative controls and increasing pollutant loading while remaining in compliance. While re-evaluating the current limits, I analyzed whether the new discharge limits should be mass-based or concentration-based. I found that managing pollutants on a mass basis was the most effective. The mass-balance analysis enables LLNL 
to understand the amount of pollutant loading coming from each internal discharge source as compared to B196 effluent loading. If LLNL is able to change their compliance to a mass-based set of limits, mass loading could be monitored at B196 and as well as internally. The model will also demonstrate that the newly determined internal discharge limits will protect the LWRP's treatment processes.

Some of the major conclusions from my SERS project include the following:

- Compared to the other POTWs that discharge to the EBDA pipeline, the LWRP's limits are the most restrictive; and they have the capacity to treat more waste than they currently are receiving.

- As shown in Figure 3, the LWRP has the available headworks loading to allocate to LLNL.

- Most LLNL discharge concentrations are well below the B196 effluent pollutant limitations; and there have not been any NOVs based on the daily discharge limits since 1989 .

- There have been no metals compliance risks at present internal guidelines.

- The existing limits are too conservative.

- New innovative ways to model LLNL's wastewater are needed.

- Modeling will assist in evaluating discharges around the lab.

- Once the internal limits are revised to phase 1 levels, a more cost-effective approach for managing wastewater discharges will result. 
Table 1

Table 2: Previous Three Months of Weekly LLNL Effluent Samples: Metals Concentrations in mg/L

\begin{tabular}{|c|c|c|c|c|c|c|c|c|c|c|c|c|}
\hline Month & $\mathrm{Ag} \quad 1$ & A & As & $\mathrm{Be}$ & $\mathrm{Cd}$ & $\mathrm{Cr}$ & $\mathrm{Cu}$ & $\mathrm{Fe}$ & $\mathrm{Hg}$ & $\mathrm{Ni}$ & $\mathrm{Pb}$ & $\mathrm{Zn}$ \\
\hline \multicolumn{13}{|l|}{ November 1995} \\
\hline Oct $30-$ Nov 5 & $<0.010$ & 0.92 & 0.0020 & $<0.0005$ & $<0.005$ & 0.018 & 0.12 & 1.8 & 0.0003 & 0.005 & 0.004 & 0.25 \\
\hline November $6,8-12^{a}$ & 0.015 & 0.56 & 0.0029 & $<0.0005$ & $<0.005$ & 0.016 & 0.12 & 1.3 & 0.0005 & 0.006 & 0.017 & 0.20 \\
\hline November $7^{b}$ & 0.013 & 1.40 & 0.0021 & $<0.0005$ & $<0.005$ & 0.020 & 0.16 & 2.7 & 0.0008 & 0.006 & 0.010 & 0.32 \\
\hline November 13-19 & $<0.010$ & 0.47 & 0.0024 & $<0.0005$ & $<0.005$ & $<0.010$ & 0.12 & 1.1 & $<0.0002$ & 0.007 & 0.023 & 0.24 \\
\hline November 20-26 & $<0.010$ & 0.45 & 0.0026 & $<0.0005$ & $<0.005$ & $<0.010$ & $<0.010$ & 1.1 & $<0.0002$ & $<0.005$ & 0.017 & 0.27 \\
\hline \multicolumn{13}{|l|}{ December 1995} \\
\hline Nov 27-Dec 3 & $<0.010$ & $<0.20$ & $<0.0020$ & $<0.0005$ & $<0.005$ & $<0.010$ & 0.014 & 0.72 & 0.0004 & 0.030 & 0.017 & 0.18 \\
\hline December $4-5,7-10^{a}, c$ & 0.007 & 1.9 & $<0.005$ & $<0.0005$ & $<0.001$ & 0.015 & 0.21 & 1.2 & $<0.0002$ & $<0.005$ & 0.095 & 0.29 \\
\hline December $\sigma^{b}, c$ & $<0.005$ & 0.6 & $<0.005$ & $<0.0005$ & $<0.001$ & 0.020 & 0.12 & 0.87 & $<0.0002$ & $<0.005$ & 0.030 & 0.18 \\
\hline December $11-17 \mathrm{C}$ & 0.006 & 1.4 & $<0.005$ & $<0.0005$ & $<0.001$ & 0.023 & 0.13 & 2.2 & 0.0003 & $<0.005$ & 0.028 & 0.28 \\
\hline December $18-24 \mathrm{C}$ & $<0.005$ & 0.8 & $<0.005$ & $<0.0005$ & $<0.001$ & $<0.001$ & 0.10 & 1.6 & 0.0002 & $<0.005$ & 0.010 & 0.22 \\
\hline December 25-31 & $<0.010$ & 1.3 & 0.0055 & $<0.0005$ & $<0.005$ & $<0.010$ & 0.32 & 2.1 & $<0.0002$ & 0.012 & 0.019 & 1.0 \\
\hline \multicolumn{13}{|l|}{ January 1995} \\
\hline $\operatorname{Jan} 1-3,5-7^{a}$ & $<0.010$ & 0.66 & 0.0031 & $<0.0005$ & $<0.005$ & $<0.010$ & 0.11 & 1.6 & $<0.0002$ & $<0.005$ & 0.012 & 0.22 \\
\hline January $4^{b}$ & 0.036 & 2.1 & 0.0044 & $<0.0005$ & $<0.005$ & 0.040 & 0.26 & 4.6 & 0.0003 & 0.009 & 0.044 & 0.54 \\
\hline January 8-14 & 0.014 & 0.74 & $<0.0020$ & $<0.0005$ & $<0.005$ & 0.021 & 0.13 & 2.0 & 0.0006 & 0.011 & 0.020 & 0.27 \\
\hline January 15-21 & $<0.010$ & 0.46 & 0.0060 & $<0.0005$ & $<0.005$ & 0.011 & 0.099 & 1.3 & 0.0003 & $<0.005$ & 0.017 & 0.23 \\
\hline January 22-28 & 0.013 & 1.0 & $<0.0020$ & $<0.0005$ & $<0.005$ & 0.035 & 0.082 & 1.5 & 0.0003 & 0.009 & 0.012 & 0.21 \\
\hline EPLd & 0.2 & N/A & 0.06 & N/A & 0.14 & 0.62 & 1.0 & $N / A$ & 0.01 & 0.61 & 0.2 & 3.0 \\
\hline
\end{tabular}

a Sampling for these weeks omitted one day because the sampling equipment was devoted to the monthly sample.

b Results from the monthly composite sample. Effluent from this date is not included in the normal weekly composite sample because the sampling equipment was devoted to the monthly sample. These results are included to complete the reporting for that week.

c LLNL is reporting the analytical results from its secondary, rather than primary, contract analytical laboratory. LLNL sent these samples for metals analysis to the secondary contract analytical laboratory at the request of the primary contract analytical laboratory.

d EPL is the Effluent Pollutant Limitation (LLNL's 1995-1996 Wastewater Discharge Permit). 
Table 2

\section{MASS BALANCE ANALYSIS}

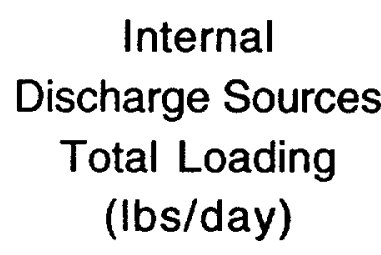

0.0095

0.0046

0.0184

0.1121

0.0267

0.0026

0.0182

0.0307

0.384

\author{
B196 \\ Effluent \\ Total Loading \\ (Ibs/day)
}

$\%$ Difference

0.0048

99.40

57.48

46.27

56.91

48.37

6.82

15.85

12.89

32.68 


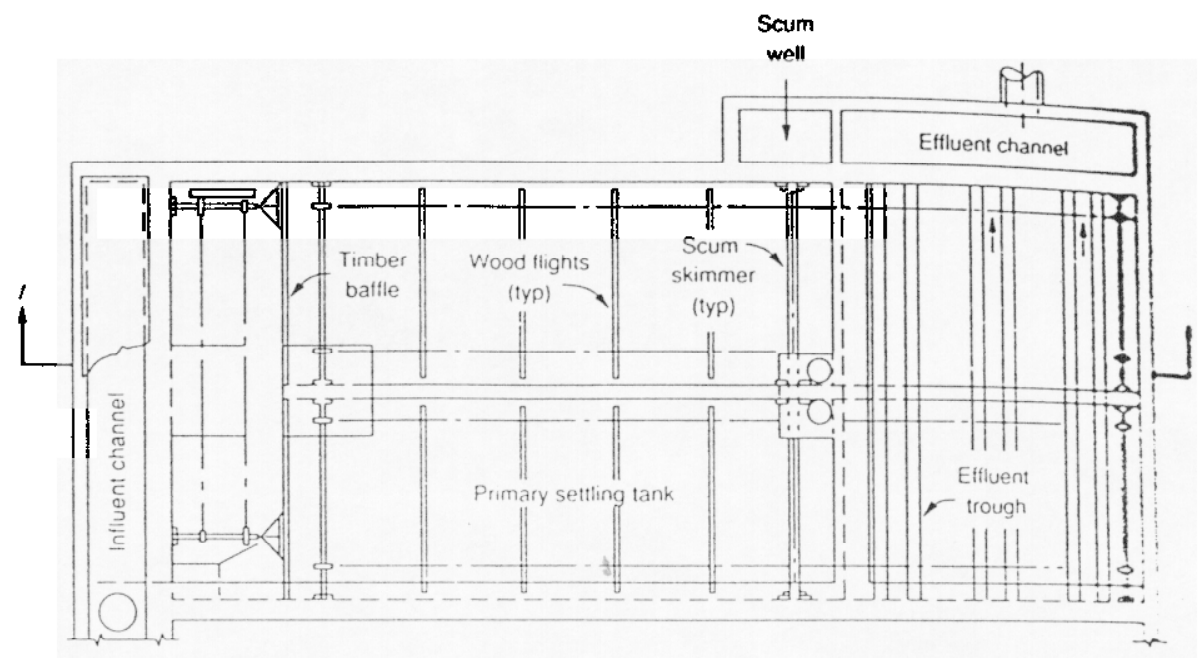

(a)

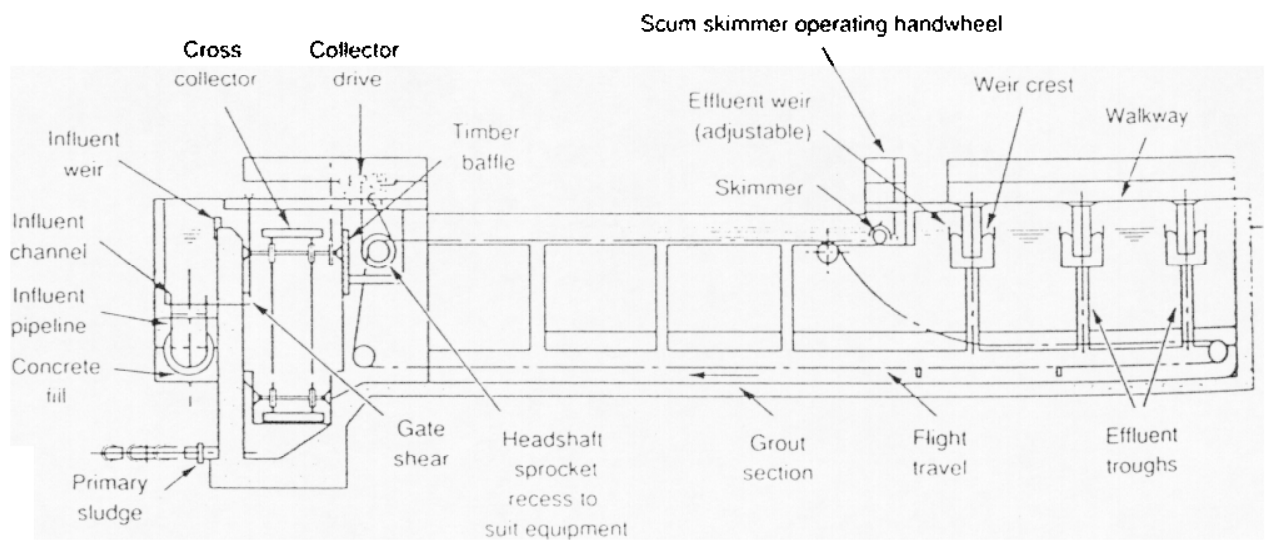

(b)

FIGURE!

Typical rectangular primary sedimentation tank

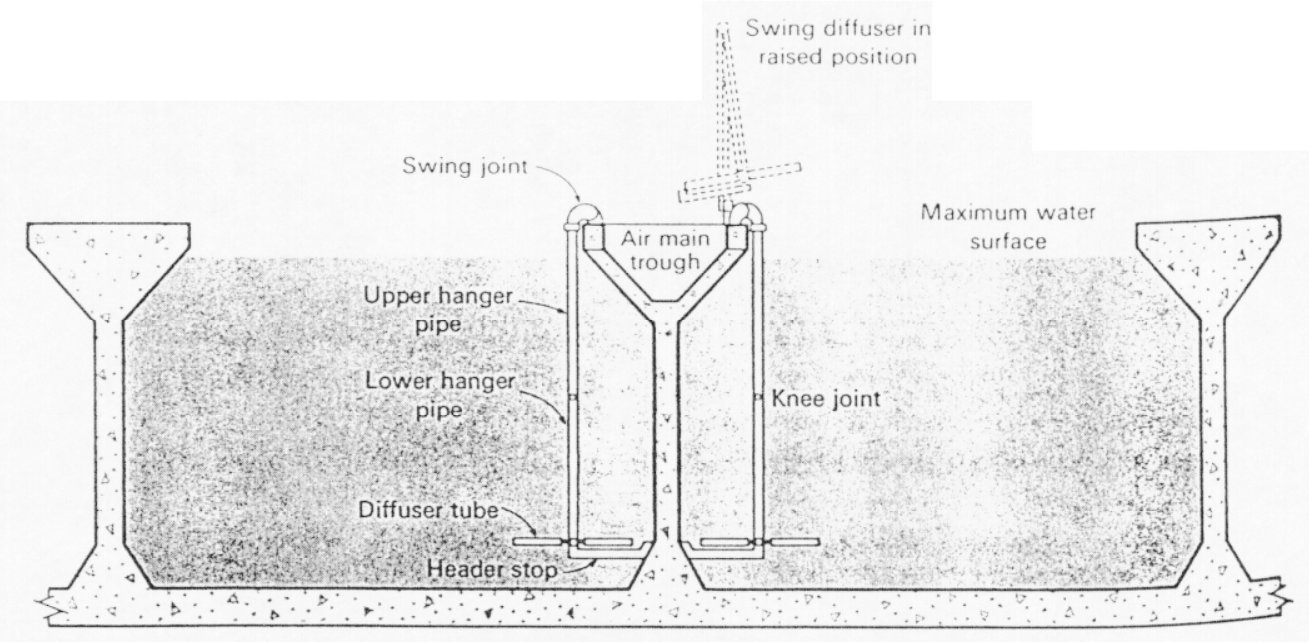

FIGURE 2

Cross section of a typical activated-sludge aeration tank using a porous tube diffused-air system. 
Figure 3

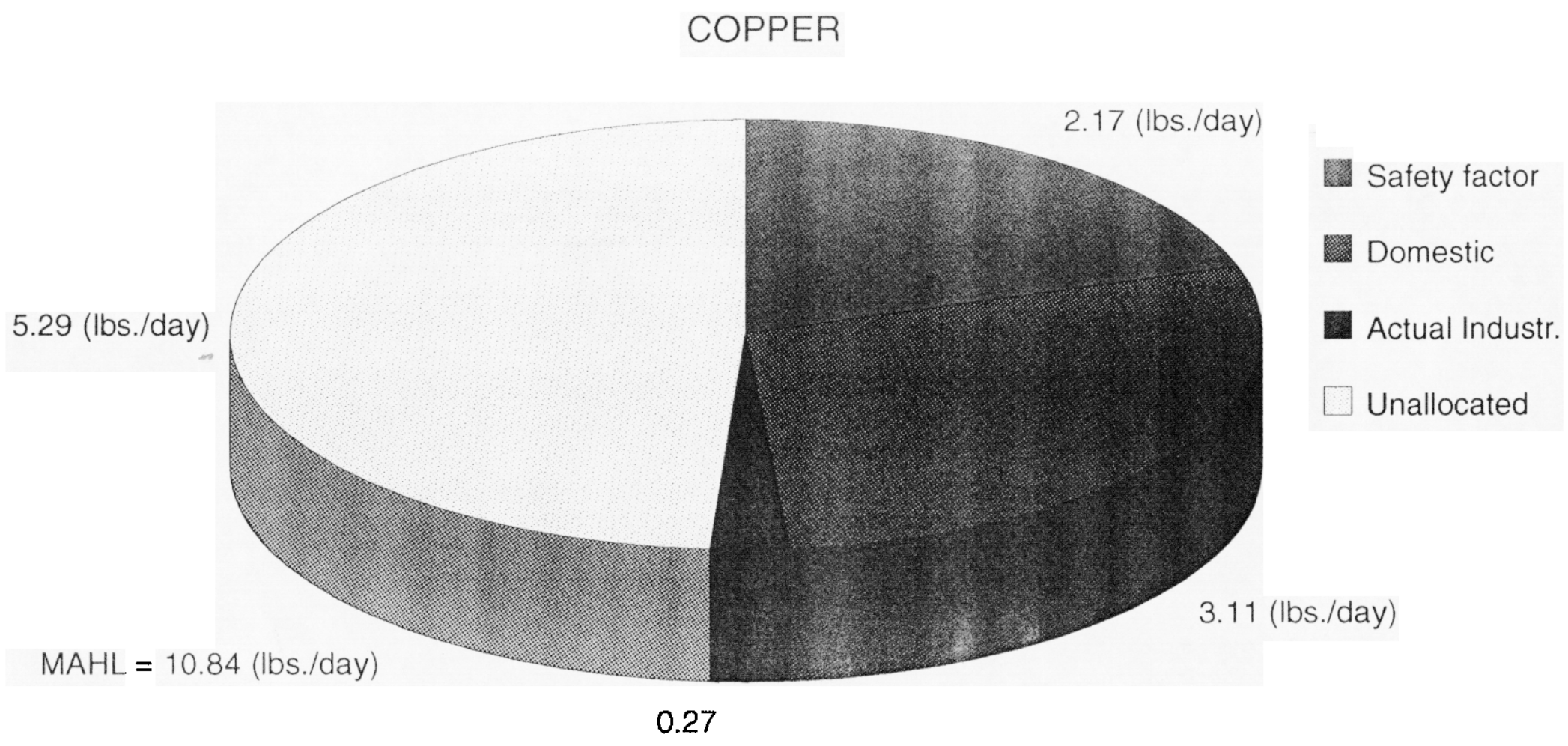


Figure 4

AVAIL. LLNL LOADING vs. ACTUAL LLNL LOADING

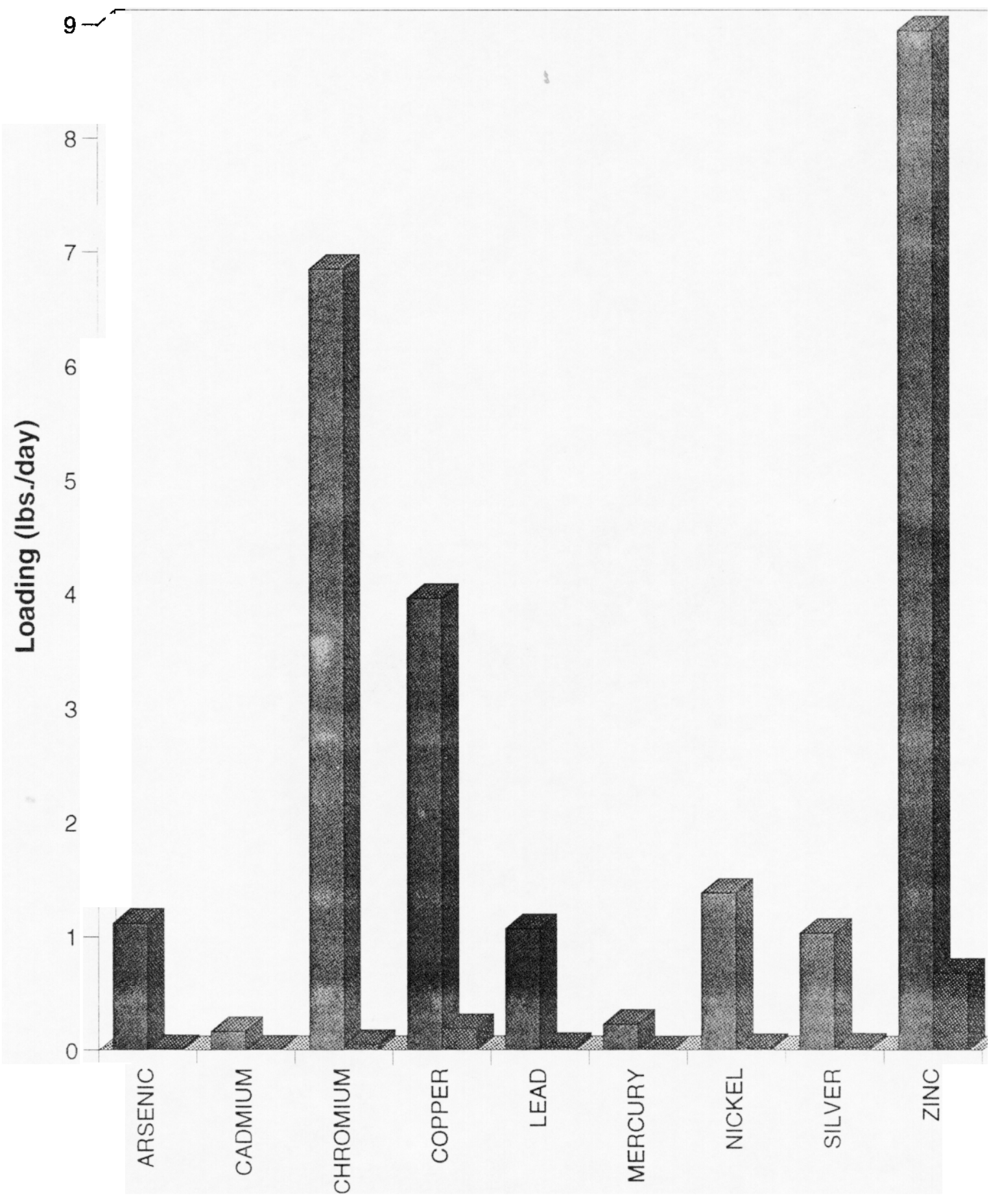

Page 15 


\section{POTW'S DISCHARGE LIMITS COMPARISON}

$\begin{array}{ccccccc}\text { Pollutant } & \begin{array}{c}\text { City of } \\ \text { Livermore } \\ (\mathrm{mg} / \mathrm{l})\end{array} & \begin{array}{c}\text { Dublin-San } \\ \text { Ramon } \\ (\mathrm{mg} / \mathrm{l})\end{array} & \begin{array}{c}\text { City of } \\ \text { Hayward } \\ (\mathrm{mg} / \mathrm{l})\end{array} & \begin{array}{c}\text { Union Sant. } \\ \text { District } \\ (\mathrm{mg} / \mathrm{l})\end{array} & \begin{array}{c}\text { Oro Loma } \\ \text { Sant. Dist. } \\ (\mathrm{mg} / \mathrm{l})\end{array} & \begin{array}{c}\text { San Leandro } \\ \text { Sant. Dist. } \\ (\mathrm{mg} / \mathrm{l})\end{array} \\ \text { Arsenic } & 0.06 & 1 & 1 & 0.35 & 0.8 & 0.1 \\ \text { Cadmium } & 0.14 & 1 & 0.2 & 0.2 & 2.2 & 0.5 \\ \text { Chromium } & 0.62 & 5 & 2 & 2 & 1.4 & 1 \\ \text { Copper } & 1 & 10 & 2 & 2 & 2 & 2 \\ \text { Cyanide } & 0.04 & 1 & 0.6 & 0.65 & 0.9 & 0.5 \\ \text { Lead } & 0.2 & 2 & 1 & 1 & 1 & 0.8 \\ \text { Mercury } & 0.01 & 0.5 & 0.01 & 0.01 & 0.0095 & 0.01 \\ \text { Nickel } & 0.61 & 5 & 1 & 1 & 1 & 0.5 \\ \text { Silver } & 0.2 & 2 & 0.5 & 0.5 & 2.2 & 0.6 \\ \text { Zinc } & 3 & 10 & 3 & 3 & 3 & 3\end{array}$




\section{3-Phase Internal Limits Comparison}

\begin{tabular}{|c|c|c|c|c|c|c|}
\hline & $\begin{array}{c}\text { Current } \\
\text { Internal Limits } \\
(\mathrm{mg} / \mathrm{l})\end{array}$ & $\begin{array}{c}\text { Phase } 1 \\
\text { Proposed } \\
\text { Internal limits } \\
(\mathrm{mg} / \mathrm{l})\end{array}$ & Phase 2 & Phase 3 & $\begin{array}{l}\text { B196 } \\
\text { Current limits } \\
\text { (mg/l) }\end{array}$ & $\begin{array}{c}\text { Haz. Waste } \\
\text { Limits } \\
(\mathrm{mg} / \mathrm{l})\end{array}$ \\
\hline Arsenic & & 3 & 3 & 10 & 0.06 & 5 \\
\hline Cadmium & 0.9 & 0.9 & 5 & 3 & 0.14 & 1 \\
\hline Chromium & 4.9 & 4.9 & 25 & 48 & 0.62 & 5 \\
\hline Copper & 10 & 24.9 & 25 & 33 & 1 & 25 \\
\hline Lead & 4.9 & 4.9 & 7 & 9 & 0.2 & 5 \\
\hline Mercury & 0.05 & 0.19 & 0.3 & 2 & 0.01 & 0.2 \\
\hline Nickel & 5 & 19 & 20 & 17 & 0.61 & 20 \\
\hline Silver & 1 & 4.9 & 6 & 9 & 0.2 & 5 \\
\hline Zinc & 15 & 65 & 65 & 65 & 3 & 250 \\
\hline
\end{tabular}




\section{REFERENCES}

Gallegos, Gretchen, et. al. 1994. Environmental Report 1994, Lawrence Livermore National Laboratory, Livermore, CA (UCRL50027-94).

Grandfield, C. H. 1989. Guidelines for Discharges to the SanitarySewer System, Lawrence Livermore National Laboratory, Livermore, CA (UCAR-10235).

Metcalf \& Eddy, Inc. Wastewater Engineering: Treatment. Disposal. and Reuse. McGraw-Hill Publishing Co. 3rd Edition. 1991.

Eisenberg, Olivieri, and Associates. Comments From Review Of LLNL Internal Memoranda Regarding Retention Tank Discharge Criteria. November 19, 1988.

EPA. Supplemental Manual On the Development and Implementation Of Local Discharge Limits Under the Pretreatment Program. May 1991.

EPA. PRELIM Version 4.0 User's Guide. Documentation For the EPA Computer Program For Development Of Local Discharge Limitations Under the Pretreatment Program. May 1991. 
.. 


\title{
TRANSPORT IN POROUS MEDIA*
}

\author{
Eric Dickenson \\ University of California at Davis \\ Lawrence Livermore National Laboratory \\ Livermore, Califormia 94550
}

\begin{abstract}
May 8, 1996
Prepare in partial fulfillment of the requirements of the Science and Engineering Research Semester under the direction of Dr. Mehdi Rashidi, Research Mentor, in the Lawrence Livermore National Laboratory.

* This research was supported in part by an appointment to the U.S. Department of Energy Science and Engineering Research Semester (hereinafter called SERS) program administrated by LLNL under Contract W-7405-Eng-48 with Lawrence Livermore National Laboratory
\end{abstract}




\title{
TRANSPORT IN POROUS MEDIA
}

\author{
Eric Dickenson
}

Environmental Programs Directorate

\begin{abstract}
A novel nonintrusive fluorescence imaging technique is used to study microscopic transport within porous media. The system consists of a column packed with heterogeneous-transparent particles and a refractive index-matched aqueous fluid seeded with fluorescent tracer particles or an organic dye. The flow through the column is illuminated by a planar sheet of laser beam and details of flow and transport through the porous regions can be observed microscopically and qualitative and quantitative transport information can be obtained. Various geometric, flow, and concentration quantities can be determined over a three dimensional volume within the column. The quantities include local and volumetrically averaged porosities, velocity and concentration fields, microscopic and volumetrically averaged dispersive fluxes and the dispersion coefficient. The qualitative and quantitative results will provide a better understanding for modeling of transport in porous media.
\end{abstract}




\section{TABLE OF CONTENTS}

Page

I. Introduction ........................................................................... 1

II. Experimental Methods ..................................................................... 2

Table 1. .Experimental Conditions .................................................... 2

Figure 1. Experimental Setup .............................................................. 3

Figure 2. Fluorescence Imaging Configuration ...................................... 4

Figure 3. Intensities of dye concentrations

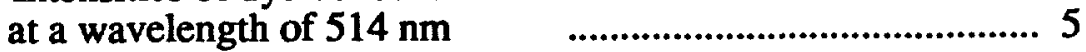

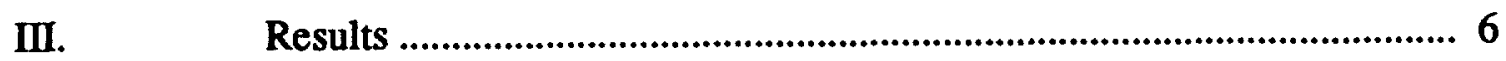

IV. Discussion .................................................................................. 6

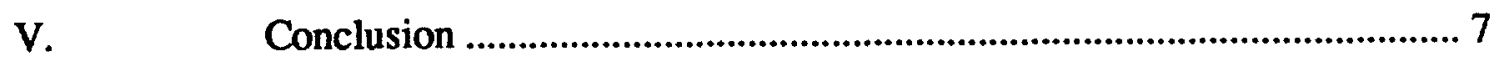

VI. References ................................................................................ 8 


\section{INTRODUCTION}

The study of transport in porous media is of great importance in numerous industrial, environmental, and biological processes. The study has potential applications in series of chemical engineering processes, soil physics and contamination problems, petroleum recovery techniques, and biomedical systems.

This research is a combined theoretical and experimental measurement effort in order to improve our understanding of chemical transport in soils and other porous media. There have been past experimental attempts to measure macroscopic porous-medium properties (Schwartz and Smith, 1953; Harleman and Rumer, 1965; Han et. al., 1985). However, little research has been devoted to experimental observation and characterization of processes within the pore spaces themselves. Microscopic experimental observations of flow and transport behavior in natural, three-dimensional systems, are essentially nonexistent because of the difficulty of obtaining detailed dynamic measurements within the pore spaces of a real medium in a nonintrusive fashion. However, this project involves three-dimensional microscopic measurements of flow and chemical transport performed in a nonintrusive manner.

Both DOE and LLNL are interested in obtaining predictive information regarding the subsurface movement of contaminants and their treatment via bioremediation. Thus, the fundamental goal of the project is to investigate the microscale processes that govern contaminant/chemical transport in porous media to provide the basis for improved modeling of contaminant transport and treatment in natural porous systems. 


\section{EXPERIMENTAL METHODS}

Experiments are performed in a clear polymethylmethacrylate (PMMA) plastic column of a width and length of $3.0 \mathrm{~cm}$ and a height of $7.0 \mathrm{~cm}$. The column is filled with heterogeneous-transparent crystals of diameter approximately $0.15 \mathrm{~cm}$. The system is saturated with an aqueous solution containing either fluorescent-latex microshperes for velocity experiments or fluorescent-organic dye for concentration experiments. Here the fluorescent microspheres and dye are the contaminants.

Before the initiation of experiments, the refractive index for the aqueous solution and crystals are matched at approximately 1.3900 at $20.0^{\circ} \mathrm{C}$ and a wavelength of 514.5 $\mathrm{nm}$ using a refractometer and temperature control. The column is maintained at this constant temperature by being immersed in a circulating-constant-temperature bath as shown in Figure 1. The refractive index matching of the liquid and crystals allows the porous medium to be free from any scattering and refraction at the solid-liquid interfaces. As shown in Figure 1, the column is saturated with the aqueous solution using a syringe pump at a steady volumetric flow rate. The aqueous solution flows from the bottom to top of the column and the effluent flows to a tank (See Figure $1 \& 2$ ). The experiment entails performing six runs at different volumetric flow rates as shown in Table 1. Also, Table 1 lists the temperature, kinematic viscosity, crystal diameter and porosity for the six different runs.

Table 1. Experimental Conditions

\begin{tabular}{|cccccc|}
\hline Runs & $\begin{array}{c}\text { Temperature } \\
\left({ }^{\circ} \mathrm{C}\right)\end{array}$ & $\begin{array}{c}\text { Kinematic } \\
\text { Viscosity } \\
\left(\mathrm{cm}^{2} / \mathrm{s}\right)\end{array}$ & $\begin{array}{c}\text { Crystal } \\
\text { Diameter } \\
\equiv(\mathrm{cm})\end{array}$ & $\begin{array}{c}\text { Porosity } \\
\cong\end{array}$ & $\begin{array}{c}\text { Volumetric } \\
\text { Flow Rate } \\
\left(\mathrm{cm}^{3} / \mathrm{s}\right)\end{array}$ \\
\hline 1 & 20.0 & 0.0381 & 0.15 & 0.4 & 0.00095 \\
2 & 20.0 & 0.0381 & 0.15 & 0.4 & 0.00955 \\
3 & 20.0 & 0.0381 & 0.15 & 0.4 & 0.0955 \\
4 & 20.0 & 0.0381 & 0.15 & 0.4 & 0.192 \\
5 & 20.0 & 0.0381 & 0.15 & 0.4 & 0.382 \\
6 & 20.0 & 0.0381 & 0.15 & 0.4 & 0.765 \\
\hline
\end{tabular}




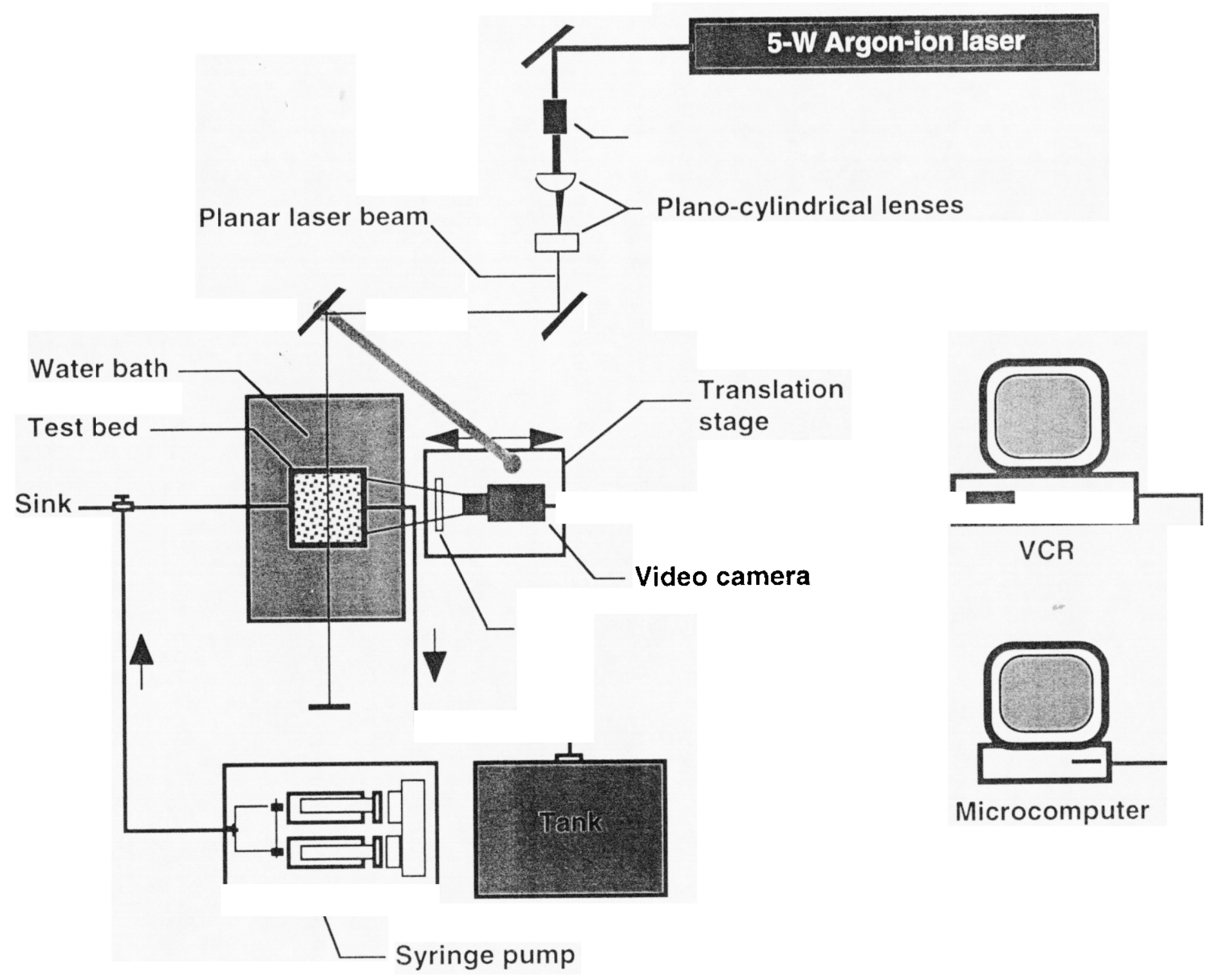

Figure 1. 


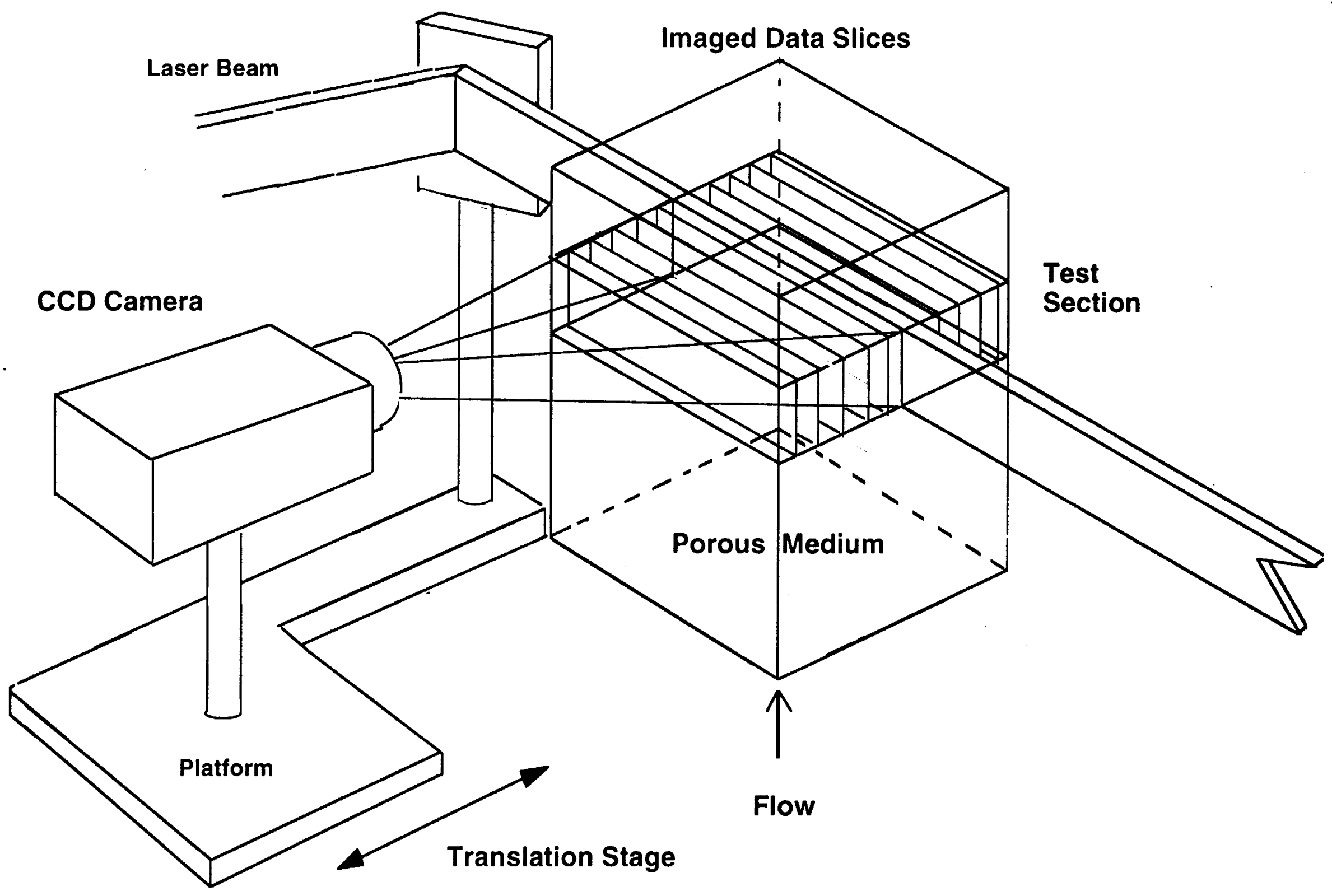

Figure 2. Flourescence Imaging Configuration 
During the experimental runs, planar sections of the observation are illuminated by a coherent Argon-ion laser beam (see Figure 2), operated at $475 \mathrm{~nm}$ for velocity measurements and $488 \mathrm{~nm}$ for concentration measurements. When illumination occurs, the fluorescent microspheres or dye fluoresces. A CCD camera records fluorescence images at 60 parallel-vertical cross-sections by sweeping back and forth across the column using a translation stage as shown in Figure 2. Approximately ten seconds is required to sweep through all 60 planes within the column. Measurements for each sweep are collected into aggregate blocks of data and used to approximate a snapshot of the system at a single time. At a given time up to 300,000 data points are taken.

The pore-velocity distribution is measured by tracking the motion of the seeded fluorescent microshperes on the 60 parallel vertical cross-sections. Following the velocimetry measurement, the fluorescent-organic dye is introduced and concentration distributions are measured. The dye fluoresces at different intensities and this intensity is correlated with the dye concentration using a fluorimeter. Differing dye concentraions are excited at $488 \mathrm{~nm}$ and the emission peaks around $514 \mathrm{~nm}$. Intensities are measured at this wavelength. Figure 3 shows a linear relationship of the fluorescent-dye intensity and dye concentrations between $0-25 \times 10^{-6} \mathrm{M}$. The dye emission peaks at around $514 \mathrm{~nm}$ when it fluoresces, so a band pass filter is used on the video camera to pass a narrow range of $514.5 \pm 5 \mathrm{~nm}$.

Figure 3. Intensities of dye concentrations at a wavelength of $514 \mathrm{~nm}$.

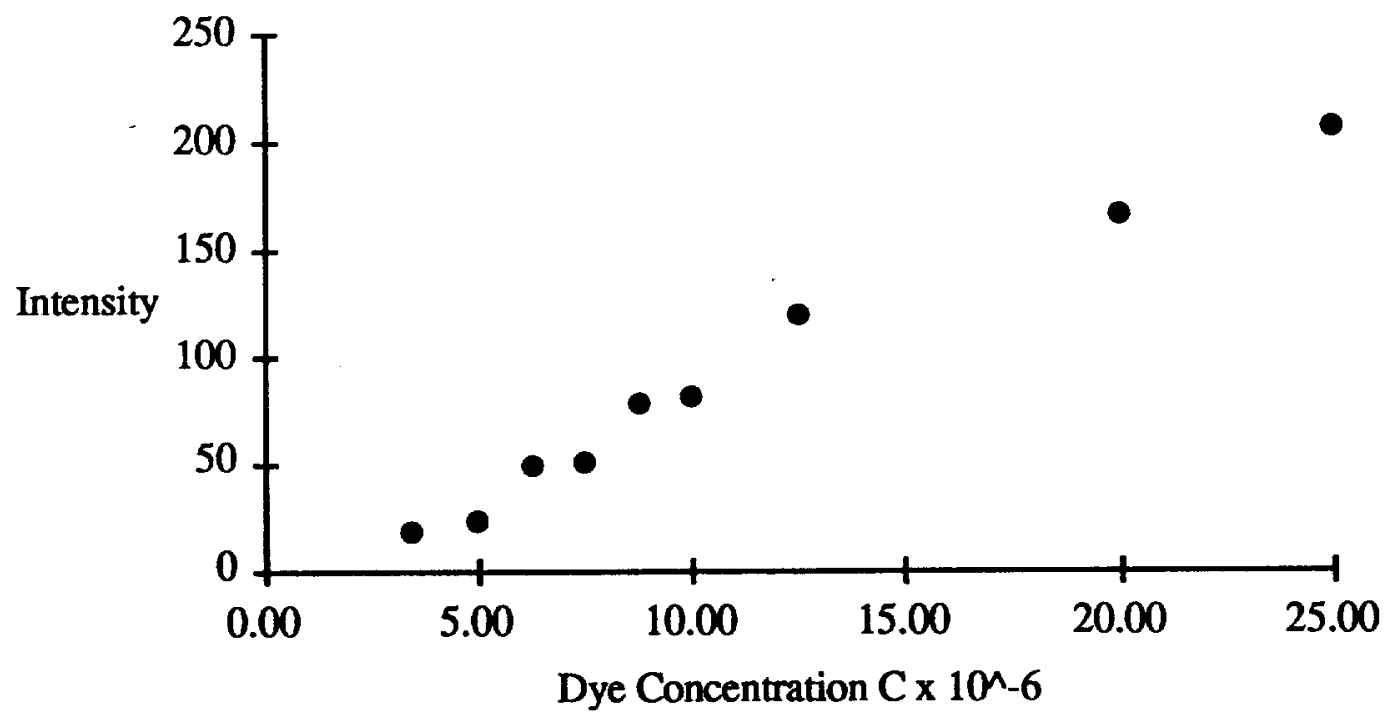


As shown in Figure 1, experimental images are recorded through the video camera by a computer controlled VCR in Hi-8 format. The velocity and concentration measurements are analyzed using image analysis programs developed in OPTMAS (BioScan) software.

\section{RESULTS}

The results of the experimental runs are in the process of being analyzed. The measured velocity and concentration fields will provide various geometry and transport quantities, such as volumetrically averaged porosities, microscopic and volumetrically averaged dispersive fluxes and the dispersion coefficient.

\section{DISCUSSION}

The liquid used in these experiments is an aqueous solution with sugar, however when the experimental project initiated the proposed liquid to be used differed. The initial liquid consisted of an aqueous solution with trifluoroethanol $\left(\mathrm{CF}_{3} \mathrm{CH}_{2} \mathrm{OH}\right)$. However, this solution causes polymethylmethacrylate (PMMA) to melt within ten minutes when in contact. Since the column is made of PMMA, the column would melt too if the trifluoroethanol was introduced to the system. The present sugar-aqueous solution does not negatively effect the PMMA column.

Since the liquid medium is water and sugar, the solution is viable for bacteria. Therefore, the measurement techniques used in the experiment will be applied to bacteria transport and this will provide new insight in bioremediation processes. 


\title{
CONCLUSION
}

\begin{abstract}
A novel nonintrusive fluorescence imaging technique is used to study microscopic transport within the porous media. Experiments were performed to measure velocity and concentration distributions. These results are being analyzed in order to evaluate volumetrically averaged porosities, velocity and concentration fields, microscopic and volumetrically averaged dispersive fluxes and the dispersion coefficient. This information will lead the next generation of transport models and make better predictions of chemical movement and biological treatment of contaminants in the subsurface.
\end{abstract}




\section{REFERENCES}

Han, N.W., Bhakta, J. and Carbonell R. G., 1985, Longitudinal and lateral dispersion in packed beds: Effect of column length and particle size distribution. AIChEJ. 31, 277-288.

Harleman, D. R. E. and Rumer, R. R., 1963, Longitudinal and lateral dispersion in an isotropic porous medium. J. Fluid Mech. 16, 385-394.

Rashidi, M., Peurrung, L., Tompson, A., and Kulp, T., 1994, Experimental Analysis of Pore-Scale Flow and Transport in Porous Media. Water Resources Research Journal..

Schwartz, C. E. and Smith, J. M. 1953, Flow distribution in packed beds. Ind. Eng. Chem. 45, 1209-1218.

Whitaker, S., 1986, Flow in porous media I: A theoretical derivation of Darcy's law. Transport in Porous Media 1, 3-25. 



\section{Chris Dillon}

\section{Greg Lennon, Tom Slezak}

$$
\text { L-452 }
$$

Biology \& Biotechnology

Research Program

\section{L.L.N.L. Human Genome Project}

DNA Fragment Location Program 
is Introduction

Abstract

Methods

Steps to finding a mapped clone

Examples of BLAST matches

it Results

Conclusions

Bibliography

Acknowledgments 


\section{Introduction}

Many biologists have specialized in different areas of genetics. Some have specialized in sequencing the DNA, some have worked on trying to find out what it does, and some it's physical structure. The biologists who sequence only expressed DNA that comes from mRNA don't know, without doing an experiment, where the DNA belongs on the chromosomes. For many labs, including LLNL, the job of integrating information that is already in the databases about these pieces of DNA was tedious. The challenge was to make a computer program that did most of the work automatically. Could such a program be written to accomplish these tasks without the biologist having to work at it? Also, could this program accomplish this within a reasonable amount of time? 


\section{Abstract}

The Human Genome Project is an international effort to find and characterize all genes in humans including those that encode the basis for all hereditary diseases. Part of characterizing genes is determining which chromosome they are on. The purpose of my project was to develop a program to find out if a gene has already been mapped to a chromosome. So, the first thing I did was gather a pool of mapped genes from various sources. Then, I developed a program to help biologists find other fragments of DNA that might overlap with one of the mapped genes. I found that many genes could be mapped without having to do an actual experiment through the use of the computer. I concluded from this that before actual experiments are preformed to determine which chromosome a gene is on, the gene should be tested by my program to determine if it needs to be mapped. 


\section{Methods}

To find out if a strand of DNA has been mapped or not there are three steps. They are:

1. Gather all sequences like the one you wish to know about.

2. Compare all the sequences you get against a list of mapped sequences.

3. View the sequence of interest to see if the mapped sequence is really the same as the sequence of interest.

This view is a greatly simplified view of what my program does. Exactly how each step is done is explained later in this paper. 


\section{Step 1- Find all sequences like the one of interest}

Lists of similar sequences are stored in a database called dbEST

(database of Expressed Sequence Tags).1 This database is accessed by my program with SQL queries through Sybase Perl. However, this database only holds matches for a particular sequence to those sequences entered before it. We knew this, so we designed the program to first find all references to the sequence of interest and then lookup what the relationships are between sequences. Also, in our program, the user can specify a score cut off, so that some of the sequences can be eliminated from the search. This saves time because the user doesn't have to look through so many sequences and our program doesn't have to sort through as many. 
Step 2 - Compare what the results with available mapping data

Part of my research project was finding mapping data and converting it all into a standard form. This was tricky because each site has a different format. A condensed form of the data is stored here at

L.L.N.L. The data that is stored here is just enough information to point the user back to the source from which the information came and tell which chromosome it is on. Information was obtained from: dbEST (database of Expressed Sequence Tags) 1 MIT/Whitehead Institute 2 Stanford University 3 the RH (radiation hybridization) database 4 GDB (Genome database) s

The name of each related sequence from step 1 is compared against the list of mapped sequences. If the name matches then the program continues with step 3 . 


\section{Step 3 - View the sequences}

If one of the sequences makes it through steps 1 and 2 , then the sequence must be viewed by the user so that they can determine for themselves if it is a true match or not. The program BLASTN $\sigma$ is used to align the sequences. Experienced users can tell the difference between a true match and a non-match. This is especially advantageous because the user and not the computer makes the final decision. For example, the next page is an example of what we would consider to be a "true match." The page after is an example of what we would consider to be "similar but not the same" type sequence. 


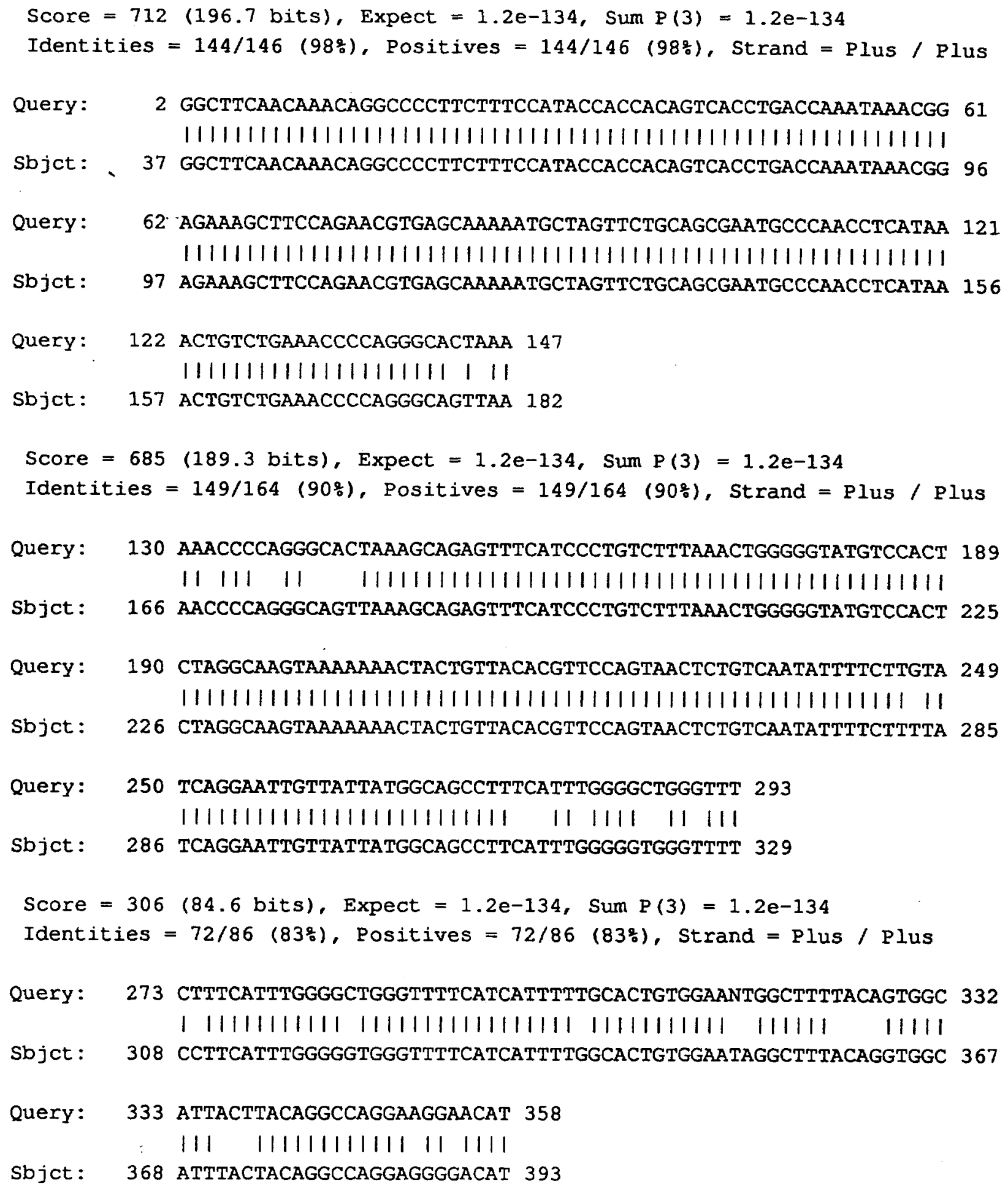




\section{Results}

Christa Prange was one of the people who tested our program. Through the use of our program, she was able to map over 50 of the 244 clones that would not have otherwise been mapped. I also found that the program works more quickly than using BLAST at N.C.B.I.? In my tests, I found that it works approximately 5 times faster. Of the approximately 400,000 sequences in the database, about 30,000 have been mapped directly by different groups. At the current success rate of about $20 \%, 80,000$ clones could be mapped by using our program. However, we found that the program can't be used when the dbEST 1 database is down. We also found the program finds most but not all related sequences depending on what you consider related. 


\section{Conclusions}

It is feasible to make such a program that can help the user to interpolate more mapping information. It can also be done in a reasonable amount of time. From this I concluded that our program should be used before mapping experiments are done and before doing the search for mapping information by hand. However, the program can definitely be improved to find more sequences thus increasing the chances of finding a match. I also think that the amount of time it takes for the program to run, could be reduced. (currently about 2 minutes) I also would recommend that use of the dbEST database be limited or cut out completely because of the amount of time the database is unaccessible. Also, we have no control over their database and can't control changes that they wish to make. So, maybe in the future we should mirror part of their data for our own use. 


\section{Bibliography}

1. see http://www.ncbi.nlm.nih.gov/dbEST/index.html for details

2. M.I.T./Whitehead

1)Dietrich, W.F. et. al. A genetic map of the mouse with 4,006 simple sequence length polymorphisms. (1994) Nature Genetics 7:220-245.

2)Copeland, N.G., D.J. Gilbert, N.A. Jenkins, J.H. Nadeau, J.T. Eppig, L.J. Maltais, J.C. Miller, W.F. Dietrich, R.G. Steen, S.E.

Lincoln, A. Weaver, D.C. Joyce, M. Merchant, M. Wessel, H. Katz, L.D. Stein, M.P. Reeve, M.J. Daly, R.D. Dredge,

A. Marquis, N. Goodman, E.S. Lander (1993) Genome Maps IV. Science 262:67.

3)Supplemented by additional markers in: Whitehead Institute/MIT Center for Genome Research, Genetic Map of the Mouse, Database Release 10, April 28, 1995.

3. see http://shgc-www.stanford.edu/rh/cdna.html for information

4. see http://www.ebi.ac.uk/srs/srsc?-info+RHDB for more information

5. see http://gdbwww.gdb.org/gdb/gdbtop.html for more information

6. see http://www.ncbi.nlm.nih.gov/BLAST/blast_help.html for more information

7. see http://www.ncbi.nlm.nih.gov/BLAST/ for more information 


\section{Acknowledgments}

Greg Lennon

Tom Slezak

Mark Wagner

Christa Prange

Joanna Albala

Kim Lieuallen

I also would like to thank the SERS program for providing the funding to make this project possible. 


\title{
Teaching Mathematics and Science using an Interactive, Web-Based Traffic Simulation*
}

\author{
Kimberly J. Drake \\ Montclair State University

\section{Lawrence Livermore National Laboratory Livermore, CA 94550}

May 10, 1996

Prepared in partial fulfillment of the requirements of the Science and Engineering Research Semester under the direction of Brian Lindow, Research Mentor, in the Lawrence Livermore National Laboratory.

*This research was supported in part by an appointment to the U.S. Department of Energy Science and Engineering Research Semester (hereinafter called SERS) program administered by LLNL under Contract W-7405-Eng-48 with Lawrence Livermore National Laboratory. 


\title{
Teaching Science Using an Interactive, Web-based Traffic Simulation
}

\begin{abstract}
The purpose of this project is to determine an internet-based method to teach mathematics and science. After evaluating possible options, we decided to develop an interactive, traffic simulation which will be accessible from the World Wide Web. At this time, a basic simulation is available along with supporting educational Web pages. In the future, a more enhanced version of the simulation will be available.
\end{abstract}




\section{Table of Contents}

Introduction. ....................................................

Methods............................................................... 5

Conclusion.................................................... 10

Bibliography................................................. 11

Appendix A................................................... 12

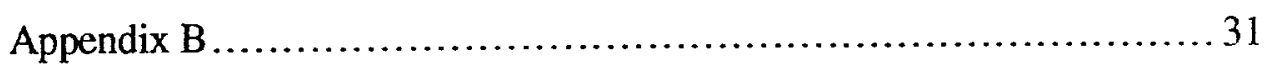




\section{Introduction}

In 1994, Chabot Observatory and Science Center was awarded a California Research and education Network (CalREN) grant to establish SMARTNet. SMARTNet is an interactive network between eight Oakland, CA community centers. SMARTNet's objective is to establish a tele-computing based infra-structure that can be used to enhance and further science education.

SMARTNet brings together schools and community centers, reaching students both in and out of the classroom. The objective of this project is to provide SMARTNet with an internet-based activity which is both educational and fun. The activity should be relevant to every student who accesses it.

An interactive, Web-based traffic simulation is an activity which is relevant to virtually every student. Students will be able to access a series of World Wide Web pages containing the simulation and supporting documents. The students will be able to set parameters and submit the simulation. A program will return pictures and data to the student detailing the simulation that the student submitted. There will be pages describing how the simulation works and the mathematical models used. 


\section{Methods}

The simulation and the CGI are written in C. Brian Lindow wrote the frame work for the simulation. I filled in the model and enhancements.

The simulation clicks through a time interval until it reaches the total number of seconds given by the user. At each iteration of time, it calculates the car's acceleration, velocity, and position. It uses several If-Then statement to decide how to calculate the new acceleration. It uses a car-following model to calculate acceleration if the car is just trailing behind the car ahead. (See appendix A for more information)

The simulation is written in $\mathrm{C}$, as is the CGI. The World Wide Web pages which comprise the user interface with the simulation are written in HTML. (See appendix B) They ask the user to give values for a number of parameters. The parameters are as follows:

\section{- Number of Cars}

Number of cars initially placed on the road. The simulation will place the number given or less, according to how many car s will fit without causing immediate collision.

\section{- Ending Time in Seconds}

Total length of time for the simulation to run. Time is measured in seconds.

\section{- Time Interval}

The simulation works by calculating positions, velocities and accelerations at different times. Time interval is the amount of time between these calculations. Give a time interval measured in seconds.

\section{- Road Length}

The length of the road in meters.

\section{- Desired Speed}

Speed that the cars will try to acquire and maintain during the simulation. Desired speed is measured in meters per second $(\mathrm{m} / \mathrm{s})$. 
- $\quad$ Current Speed

Speed of cars when they first enter the simulation. Current speed is measured in meters per second $(\mathrm{m} / \mathrm{s})$.

- Range of Speed

Interval used to randomize velocities.

- Weather

There are three choices of weather conditions: Rain, Fog, Sunny. Weather conditions are used together with Driving Style to determine braking acceleration and safe distance between cars.

- Driving Style

There are three choices of driving style: Insane, Normal, Cautious. Driving Style is used together with Weather Conditions to determine braking acceleration and safe distance between cars.

- The Picture

Users are asked to give the width and height of the resulting graph in pixels.

- The Cars

Users are asked to give the width and height of the individual cars in pixels.

The cars are a linked list of "car" structures. Each structure contains the following fields of information:

- CarNumber

A unique integer the car receives when its first added to the list. 
- Active

A Boolean variable which is initially set to 1 and is eventually set to 0 when the car's position is greater than the length of road the user sets.

- Deccel

A Boolean variable which is used to set the colors in the output map. After the car's new velocity is calculated, the old velocity is compared to the new velocity. If the old velocity is greater than the new velocity, deccel is set to one and the car will be printed red on the map.

- Accel

A Boolean variable which is used to set the colors in the output map. After the car's new velocity is calculated, the old velocity is compared to the new velocity. If the old velocity is less than the new velocity, accel is set to one and the car will be printed green on the map.

\section{- InAccident}

InAccident is a Boolean flag. If InAccident is set of 1 the car no longer changes position. Also, if InAccident is set to 1 , the car is printed in yellow on the map.

- DesireSpeed

Before the simulation is run the user is asked to provide a desired speed. At this point, all of the cars will have the same desired speed. However, future versions of the simulation will allow for different cars to have different desired speeds.

- CurrSpeed

Users are prompted for a current speed before running the simulation. Using that as an initial value, current speed is recalculated with each iteration of time.

- SpeedRange

Speed range is the value used to randomize calculated speeds. After a speed is calculated, it is randomized such that

CurrSpeed-SpeedRange $<$ New Current Speed $<$ CurrSpeed + SpeedRange 


\section{- DriveStyle}

Users are asked to provide a driving style from the choices: Insane, Normal, and Cautious. Each choice then effect accelerations used in the simulation.

\section{- DistSpeed}

DistSpeed is the speed used to determine if a car is too close to the car in front of it.

DistSpeed is set according to user-provided weather conditions and driving style.

\section{- BrakingAccel}

Braking Accel is a constant used to determine the negative acceleration of a car which is braking. BrakingAccel is set according to user-provided weather conditions and driving style.

\section{- ToTheFloor}

ToTheFloor is used to determine the positive acceleration of a car which is increasing in speed. It is set according to the user-provided weather and driving style.

\section{- Pos}

Pos is used to contain the car's position at the given interval of time.

At this time, the simulation's output is a map plotting each cars position at the end of each interval of time. The plot reflects the acceleration of the car by the color of the pixels used to plot the car. If the car's acceleration is positive during the interval of time, then the car is plotted in green. It the car's acceleration is negative during the interval of time, then the car is plotted in red. If the car's acceleration is constant, the car is plotted in black. Finally, if collision has occurred, the car is plotted in yellow. 


\section{Conclusions}

At this time the simulation is still a work in progress. While it does simulate traffic in an accurate manner, it is boring. It will not hold students' attention for any length of time. As such, it will be an ineffective teaching tool. It is also missing much of the educational WWW pages which will make it effective. However, it has the potential to be an a valuable tool both in and out of the classroom.

Much still needs to be done. First, some sort of scale must be included on the output map. It is difficult to understand as it is.

Second, more parameters should be added to the simulation. This will make it more interesting and stimulating for the students. It will also make the simulation more realistic.

Third, more educational resources need to be added. Pages explaining the interaction between position, velocity, and acceleration need to be added. All of the educational pages should include thought provoking questions. Also, other internet resources for both students and teachers need to be included. In addition, there will be a page of links to other interactive science and math activities.

Finally, at this time, cars are treated as particles. The simulation needs to be modified so that cars are treated as objects with length. 


\section{Bibliography}

Haverman, Richard. Mathematical Models. Prentice-Hall, Inc. Englwood Cliffs, NJ. 1977.

Hsu, Jende. Car Following on a Single Lane No-Passing Highway: A Simulation Model.

Modeling and Simulation. Instrument Society of America, 1974. Volume 5, part 2, 773-779. 
Figure 2. Rutherford's model of the atom (1911)

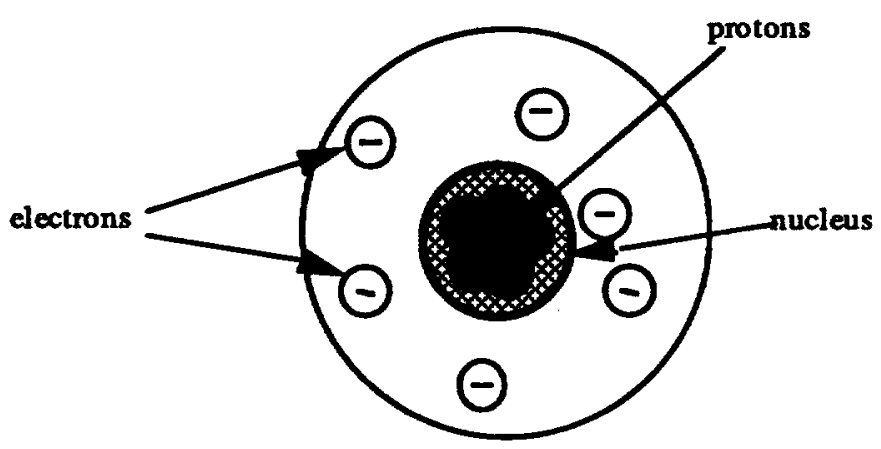

One of the most significant discoveries of modern physics was of the neutron $^{2}$ by James Chadwick in February 1932. Although the existence of the neutron was predicted earlier its discovery was delayed because of the difficulty in detecting a particle without charge. Frederic Joliot and Irene Curie-Joliot, daughter of Marie Curie, reported that "radiation from beryllium excited by alpha particles could eject protons from paraffin wax" (Boorse, 340). Chadwick, however, was the first to explain this effect as due to this neutral particle. ${ }^{3}$ He proposed that the radiation consisted of particles, with no charge (and a mass equal to that of protons). His discovery lead to the field of nuclear physics, which lead to the development of fission research and the atomic bomb.

Figure 3. Bohr's Planetary Model of the Atom (1936)

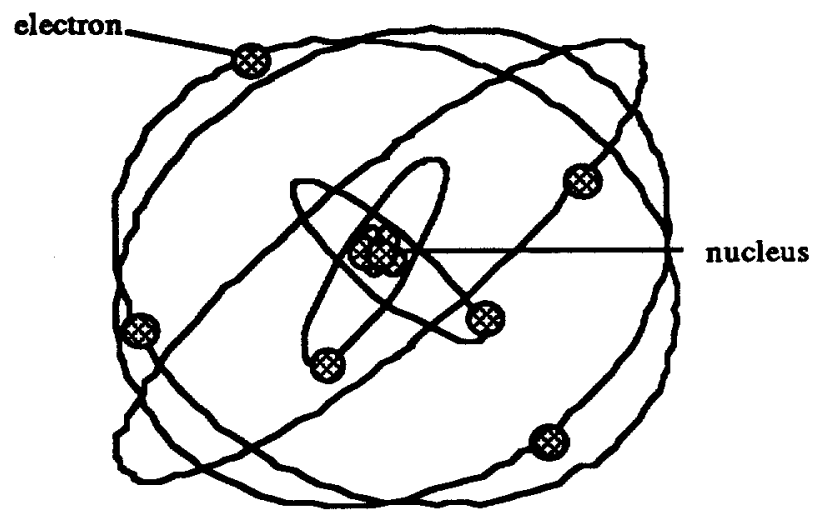

Six years after Chadwick's experiments revealed the neutron, Otto Hahn and Fritz Strassman in Germany split the uranium atom on December 19, 1938. However, Hahn did not know what to make of his experimental results, so he consulted his good friend and colleague Lise Meitner, who had left Germany and was now living in Sweden. On Christmas Eve 1938, Meitner, on vacation with her nephew, Otto Frisch, had a brilliant idea about Hahn's experiments bombarding uranium with neutrons, which resulted in the detection of barium and other isotopes. ${ }^{4}$ 
Using Bohr's liquid drop model of the atom ${ }^{5}$ and Einstein's $E=m c^{2}$ formula, Meitner developed the idea of nuclear fission. She postulated that, under the right conditions, fission occurs when the atom (mother particle) absorbs a neutron and splits into two smaller atoms (daughter particles) and releasing additional neutrons. These additional neutrons are absorbed by other uranium nuclei, which in turn, each split into two particles and the process repeats itself over and over again--a chain reaction.

Figure 4. Nuclear fission

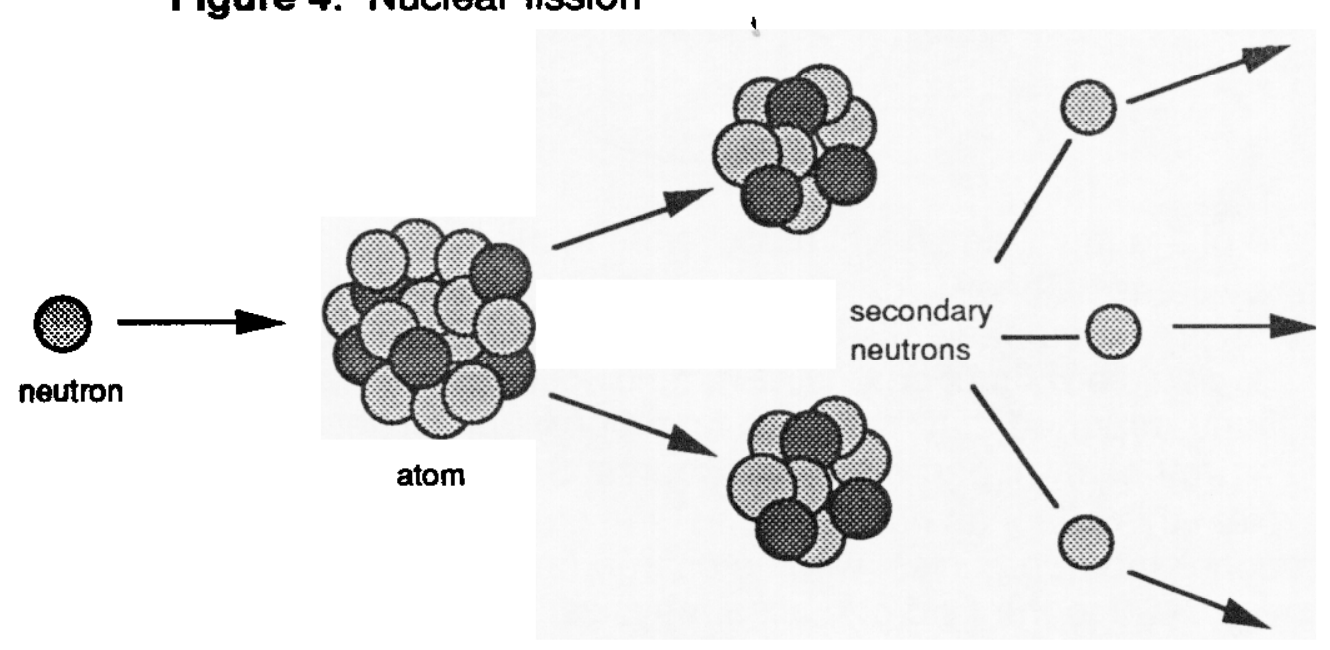

Nuclear Fission

One thing that was puzzling to them is why doesn't this chain reaction occur in nature since the earth contains uranium? They speculated that the reason may be that not enough secondary neutrons are emitted thus preventing a sustained natural chain reaction; or, perhaps only certain isotopes of natural uranium, which is mostly $238 \mathrm{U}$, fission; or, both reasons may be valid.

\section{WWII (1939 - 1945): Making the Bomb}

Once nuclear fission was confirmed by Frisch's mathematical calculations and the news released, scientists all over the world were scrambling to conduct their own experiments. Governments of most of the industrialized nations contributed in some form to the research and development of harnessing atomic energy. By the middle of World War II in 1942, the governments in Japan, Germany, the Soviet Union, and France were independently funding atomic research. When American scientists did not respond to an article in the Russian Physical Review (June 1940), the Soviet Union was convinced that the Americans knew about nuclear fission and were secretly working on a project. 
U.S. officials were hesitant at first to keep nuclear research secret in order to beat the Germans, who were most likely conducting similar research with the intention of building an atomic bomb. Louis Sziland, a Hungarian physicist who immigrated to the U.S. to escape Hitler's persecution of the Jews, warned President Roosevelt via a letter from Albert Einstein in July 1939 of the deadly potential of an atomic bomb and the enormous consequences if it were to get into the hands of the Germans. Through the persuasion of a mutual party, Alexander Sachs ${ }^{6}$, Roosevelt agreed and initiated government support of atomic bomb research and development by agreeing to contribute $\$ 6,000$ to fund the research on October 11,1939 .

The Advisory Committee on Uranium had its first meeting on October 21, 1939. However, progress was slow as White House officials did not feel the matter to be urgent. Roosevelt held the report from the Uranium Committee, which focused on using nuclear energy as a source of power for submarines, without acting on it well into 1940. Growing impatient, Szilard persuaded Einstein ${ }^{7}$ to write another letter to Roosevelt to be delivered again by Sachs. Roosevelt asked for another report, so the Uranium Committee met again on April 27. Finally, Columbia University was paid $\$ 6,000$ to purchase graphite, necessary for Enrico Fermi to conduct a uraniumgraphite experiment to prove that a chain reaction was possible.

By 1941, the National Academy of Science (NAS), directed by Arthur H. Compton, was involved in the decision-making process. Compton submitted a report to Roosevelt, recommending that the U.S. government commit resources to the research and development of an atomic bomb. The report was backed up by information received on the British and German independent atomic research. Roosevelt agreed and his decision came one day before the U.S. entered WWII with the bombing of Pearl Harbor by the Japanese on December 7, 1941.

With the appointment of General Leslie Richard Groves (of the Army Corps of Engineers) as director, the Manhattan Project was made official in September 1942. Groves' initial headquarters was in Manhattan, New York (hence the name Manhattan Project). He was unhappy about the numerous locations where atomic research was being conducted, in particular, Fermi at Chicago and Lawrence at UC Berkeley so Groves directed a few of his men to select a new central site for the project. It was Berkeley physicist, Robert Oppenheimer, who recommended Los Alamos, New Mexico. The location was perfect: remote, high above neighboring towns allowing for tight security, and a mild climate. The land was purchased in November 1942 and plans were made for the move. By then, Oppenheimer had been appointed civilian director of Los Alamos. It was his responsibility to recruit the talented scientists needed to design an atomic bomb.

On March 15, 1943, the first group of scientists and their families arrived at the site, which was to be their home for the duration of the war. These men either uprooted their families or left them for an extended period of time and relocated to a desert community $\mathbf{4 0}$ miles from the nearest town. In addition, they were aware that upon moving to Los Alamos visiting relatives was out of the question as no one was allowed to leave for personal reasons. Enrico Fermi was the only one allowed to leave for personal reasons when his wife was hospitalized for cancer in a nearby town. 
During the spring of 1945, the war was coming to an end. The Germans surrendered to the U.S. and its allies on May 7, 1945. After the war in Europe was over the U.S. discovered that the Germans were not as advanced in atomic research as once suspected. With the European theater restored to peace, the U.S. turned its attention to the Pacific theater, to Japan.

Meanwhile, at Los Alamos, scientists had successfully created the first atomic bomb. A test of the bomb, the Trinity test, was conducted at 5:29:45 in the morning on July 16, 1945 sixty miles northwest of an uninhabited area near Alamogordo, New Mexico, 210 miles south of Los Alamos. The amount of energy released from the fission reaction was far beyond that possible with conventional weapons. It was estimated that 18 kilotons of energy (equivalent to $36,000,000$ pound of TNT) was released in the Trinity test.

After the success at Alamogordo, government officials, military planners, and scientists decided on the location of the targets in Japan: among those selected was Hiroshima. ${ }^{8}$ The targets were selected: (1) because these cities had not be exposed to any previous bombing raids, thus making it easier to observe and make quantitative measurements; (2) no U.S. prisoners of war were known to be held there; and (3) President Truman, like his predecessor, wanted to end the war as soon as possible and was convinced that the Japanese would immediately surrender. After the Enola Gay B-29 bomber dropped its payload, Little Boy, onto Hiroshima on August 6, the Japanese refused to surrender and could not believe that one bomb could cause so much death and destruction. Wanting to give the impression of an unlimited atomic bomb arsenal, Truman authorized the release of a second atomic bomb, the last to be used as a weapon of war. Nagasaki was targeted just three days later with a bomb of a different design called Fat Man. Japan surrendered to the U.S. unconditionally on August 15, 1945. 


\section{Part II: Nuclear Weapon Development Post-WWII}

\section{Cold War (1946 - 1991)}

The conclusion of WWII did not end the research in atomic/nuclear physics. The U.S. was proud of the accomplishments achieved during the Manhattan Project and ending the war with the bombing of Hiroshima and Nagasaki. After WWII the U.S. was the most powerful country in the world. It held the secrets to constructing the most destructive weapon man had ever conceived. In July 1946, the U.S. began nuclear testing on the island of Bikini Atoll in the Pacific Ocean, marking the beginning of a 20 year testing program. European allies were not far behind. In August 1949, the Soviet Union detonated its first atomic bomb. The Cold War between the U.S. and U.S.S.R. was underway.

Dissatisfied with the progress of hydrogen bomb research at Los Alamos, Edward Teller decided to establish his own research facility. In July 1952, construction began on the Radiation Laboratory in Livermore, California under the direction of Teller and Lawrence. The first hydrogen bomb was tested on November 1,1952 on the uninhabited island of Eniwetok. Mike, as the bomb was called, evaporated the island and the shock wave from the blast was detected in the basement seismograph lab at the California Institute of Technology. The estimated yield of the bomb was equivalent to 10 megatons (equivalent to $20,000,000,000$ pounds of TNT).

The race to build bigger and more destructive bombs was at full throttle between the U.S. and the Soviet Union. The arms race led to the development of a series of weapons by the U.S. and the Soviet Union including both tactical and strategic weapons and weapons used for either offensive or defensive purposes. The intercontinental ballistic missile was developed in the early fifties carrying at first one and eventually multiple nuclear warheads. In January 1954, the first nuclear submarine was launched. Soon long range missiles were able to be launched from sea. Weapons to counter other weapons included anti-ballistic, anti-aircraft, antiship, anti-submarine missiles and depth bombs. In addition to atomic bombs dropped by aircraft, tactical atomic weapons were developed and included artillery shells, short-range missiles, and even atomic land mines. The stockpiles of atomic weapons grew among the nuclear powers. The number of U.S. and the Soviet atomic weapons had grown to over sixty thousand at their peak. Finally, arms control agreements, starting in the early seventies, began to cut these arsenals down.

The closest the U.S. ever came to fighting in a nuclear war was during the Cuban missile crisis in 1962. CIA intelligence discovered the Soviet Union was installing nuclear missiles on the island of Cuba, one of its communist allies. In response, President John F. Kennedy called for the removal of the missiles or Cuba would be attacked. U.S. troops were sent to Miami, Florida and a naval blockade was established surrounding the island. Nuclear missiles were put on alert . The stalemate between Kennedy and Nikita S. Krushchev lasted for one week until the latter agreed to U.S. terms. 
By the mid-1970s, the Soviet Union had surpassed the U.S. in the number of nuclear weapons stockpiled. Fearing the spread of nuclear weapons and their eventual use on a global scale, the United Nations (U.N.) decided that an agreement must be enacted between the nations of the world (including those that have nuclear weapons and those that do not have nuclear weapons). The Nonproliferation Treaty (NPT) originally entered into force in 1970 and was renewed indefinitely by all but a few nations in 1995.

\section{Post-Cold War (1991 - present)}

The U.S. produced the fissile core for its last nuclear weapon in 1989 when construction was stopped at the Rocky Flats Plant in Colorado. Today, the U.S. does not perform research, develop new nuclear weapons, or even produce fissile materials. However, Russia continues to produce weapons and fissile material even after the break up of the Soviet Union. The nuclear weapons complex within the Soviet Union has become so expansive that many Russian citizens are dependent on these nuclear weapons facilities for employment and electrical energy.

Since the initiation of the original NPT, most parties have complied to the agreement. Some nations that didn't sign the treaty developed weapons or are trying to develop weapons. These include South Africa, India, Israel, and Pakistan. South Africa has since disbanded its program and destroyed its weapons. Still others who signed the treaty blatantly violate the agreement. Iraq and North Korea have intentions to develop nuclear weapons. Although the U.S., Russia, and the U.K. have stopped testing atomic weapons, China continues to test. On June 13, 1995, France resumed nuclear weapons testing at Mururoa Atoll in the South Pacific, but also recently promised to stop further testing.

Since the break up of the Soviet Union in 1991, three new nuclear weapon states were formed but have since agreed to eliminate their weapons. Weapongrade material has been smuggled out of Russia and sold on the black market. It has become extremely difficult for the five nuclear weapon states (United States, Russia, China, France, and United Kingdom) and the U.N. to keep track of all manufacturing of weapon grade materials and nuclear testing. The U.S. and Russia have resumed negotiations of START II (Strategic Arms Reduction Treaty) in 1995. This particular treaty if entered into force would severely limit the number of nuclear warheads to 3,200 for each country.

Although the Cold War is over, the U.S. and the rest of the world still face threat of nuclear aggression by terrorist groups or rogue countries that possess nuclear weapons. The immediate future does not foresee the complete elimination of nuclear weapons. The best means of avoiding a nuclear conflict is assuring that present nuclear weapons are secure and we limit the spread of the technology to develop them. 
References

Boorse, Henry A., Lloyd Motz, and Jefferson Hane Weaver. The Atomic Scientists: A Biographical History. New York: Wiley, 1989.

Clinton Administration Reduces Intemational Nuclear Threat. Press Release from U.S. Department of Energy, Feb. 1, 1996.

Closing the Circle on the Splitting of the Atom. U.S. Department of Energy, Jan. 1995.

Cochran, Thomas B., et al. Nuclear Weapons Data Book, Volume II: U.S. Nuclear Warhead Production. Cambridge: Ballinger, 1987.

Glasstone, Samuel. Sourcebook on Atomic Energy. Princeton: Nostrand Co., 1967.

Glasstone, Samuel and Philip J. Dolan. The Effects of Nuclear Weapons, 3rd ed. U.S. Department of Defense and U.S. Department of Energy, 1977.

Hansen, Chuck. U.S. Nuclear Weapons: The Secret History. New York: Orion, 1988.

Herron, J. Dudley, et. al. Chemistry. Lexington, MA: D. C. Heath, 1993.

Jungk, Robert. Brighter Than A Thounsand Suns. London: Golancz, 1958.

Marriott, John. International Weapon Developments, 4th ed. New York:

Pergamon, 1980.

Mayers, Teena. Understanding Nuclear Weapons and Arms Control. Arlington, VA: Arms Control Research, 1983.

Nuclear Proliferation Factbook. Congressional Research Senvice, 99th Congress. Washington, D.C.: U.S. Printing Office, 1985.

Rhodes, Richard. The Making of the Atomic Bomb. New York: Simon and Schuster, 1986.

Thomas, Dianne Stine. WWII: Time-Life Books History of the Second World War. New York: Princeton-Hall, 1989.

Zitzewitz, Paul W., et. al. Physics: Principles and Problems. New York: Glencoe, 1995. 


\section{Endnotes}

${ }^{\circ}$ One year later, Pierre was killed in a terrible street accident, leaving Marie to care for their two daughters and replace him as chair of physics at the Sorbonne.

'Rutherford postulated that the electrons moved at high speeds around the nucleus. However, this goes against the laws of physics of that time--orbiting electrons should generate energy in the form of light, thus, releasing energy and collapsing into the nucleus.

${ }^{2}$ Besides the electron and proton, the photon had already been discovered by this time. The positron, a positive electron, was discovered by Carl D. Anderson on August 2, 1932.

${ }^{3}$ Until then, scientists were performing particle bombardment experiments in which a sample, like radium, was bombarded with protons. The resulting radiation could not be explained assuming that it consisted of high-energy photons.

4At the time, Otto Frisch was residing in Copenhagen, Denmark where he was studying at Niels Bohr's institute.

${ }^{5}$ Bohr's postulated that electrons moved in fixed orbits around the nucleus. He assumed that electrons in a stable orbit around the nucleus did not radiate energy.

${ }^{8} \mathrm{Dr}$. Alexander Sachs, a biologist and national economist, was vice-president of the Leman Corporation in New York and an acquaintance of President Franklin D. Roosevelt.

${ }^{7}$ Although Einstein never participated in the Manhattan Project, he felt it his duty to wam the President of the great possibility of an atomic bomb attack by the Germans. After the war, Einstein publicly admitted he had regrets about the letters written to Roosevelt. He felt guilty about the mass destruction and loss of life in Japan.

?Three targets were selected: Kyoto, Hiroshima, and Niigata. Hiroshima was chosen because it had the largest area untouched by air bombings, thus, more data could be obtained from the aftermath. Just weeks before the bombing, U.S. government officials had several choices in deciding whether or not to bomb Hiroshima: (1) don't drop the bomb and tell Japan of its existence; (2) test the bomb in an uninhabited area near Japan; or, (3) give warning of the bombing, then drop the bomb; or, (4) no waming and drop the bomb. They chose the later. According to Rhodes, many of Japanese citizens were manufacturing aircraft parts in their homes ( Rhodes p649). 


\title{
Progress Toward the Synthesis of a Cleavable Linker Molecule: Dimethyl bis[2-[2-[2-[2-ethoxy]ethoxy] ethoxy]ethoxy] silane dibiotinylate*
}

\author{
Christine Liberatore \\ Eastern Illinois University \\ Lawrence Livermore National Laboratory \\ Livermore, California 94550

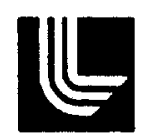

May 10, 1996

\footnotetext{
Prepare in partial fulfillment of the requirements of the Science and Engineering Research Semester under the direction of Dr. Ken Bogen, Research Mentor, in the Lawrence Livermore National Laboratory.

*This research was supported in part by an appointment to the U.S. Department of Energy Science and Engineering Research Semester (hereinafter called SERS) program administered by LLNL under Contract W-7405-Eng-48 with Lawrence Livermore National Laboratory.
} 


\section{Introduction}

The goal of this project was to develop the synthesis of a linker molecule that can be easily cleaved under mild aqueous conditions. The importance of this linker is described in a paper by Ken Bogen. The synthesis consists of two basic steps in which an intermediate is first made and isolated, followed by an esterification reaction to yield the final product. Results from my research this semester have shown that the first step is successful; however, we have not yet proven that the second step works. This paper will outline the details of the proposed synthesis, the results and tests pertaining to the synthesis, and a future plan of action.

\section{Methods}

Step 1: Synthesis of Dimethyl bis[2-[2-[2-[2-ethoxy]ethoxy]ethoxy] ethoxy] silane diol

\section{Step 1}
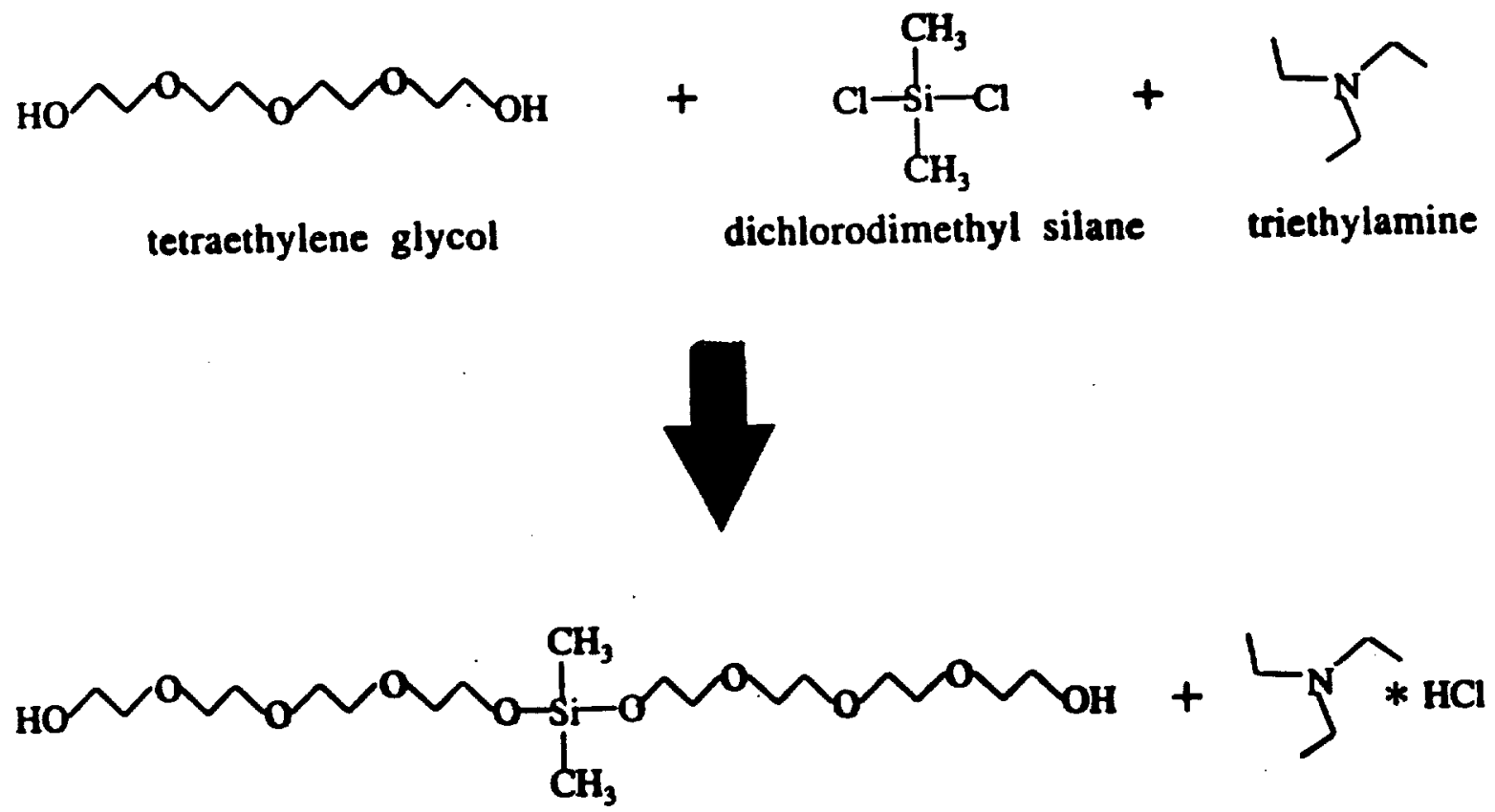


\section{Appendix A: The simulations code}

(Significant amounts of the following code were written by Brian Lindow. Modifications were made by Kimberly Drake)

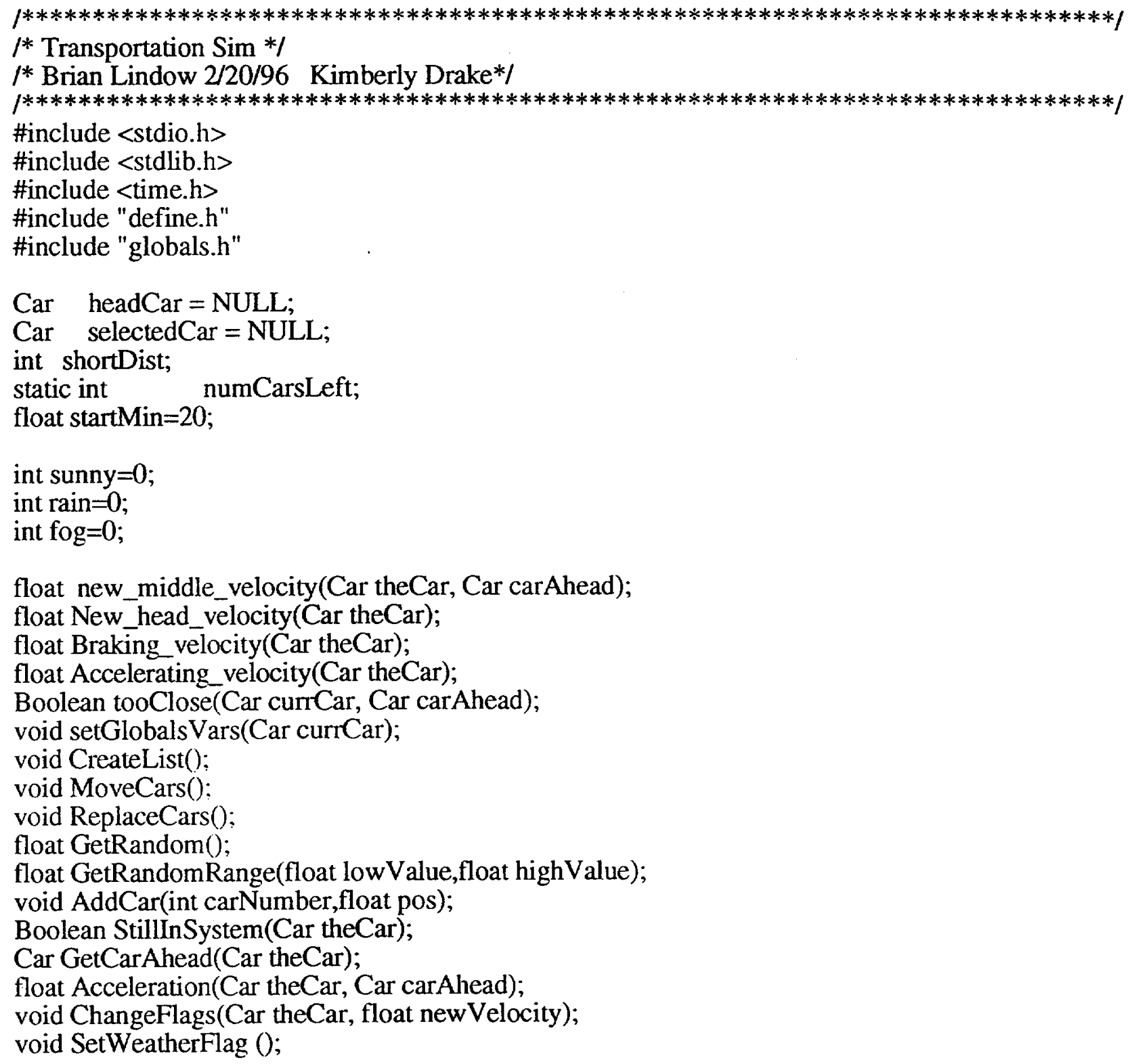




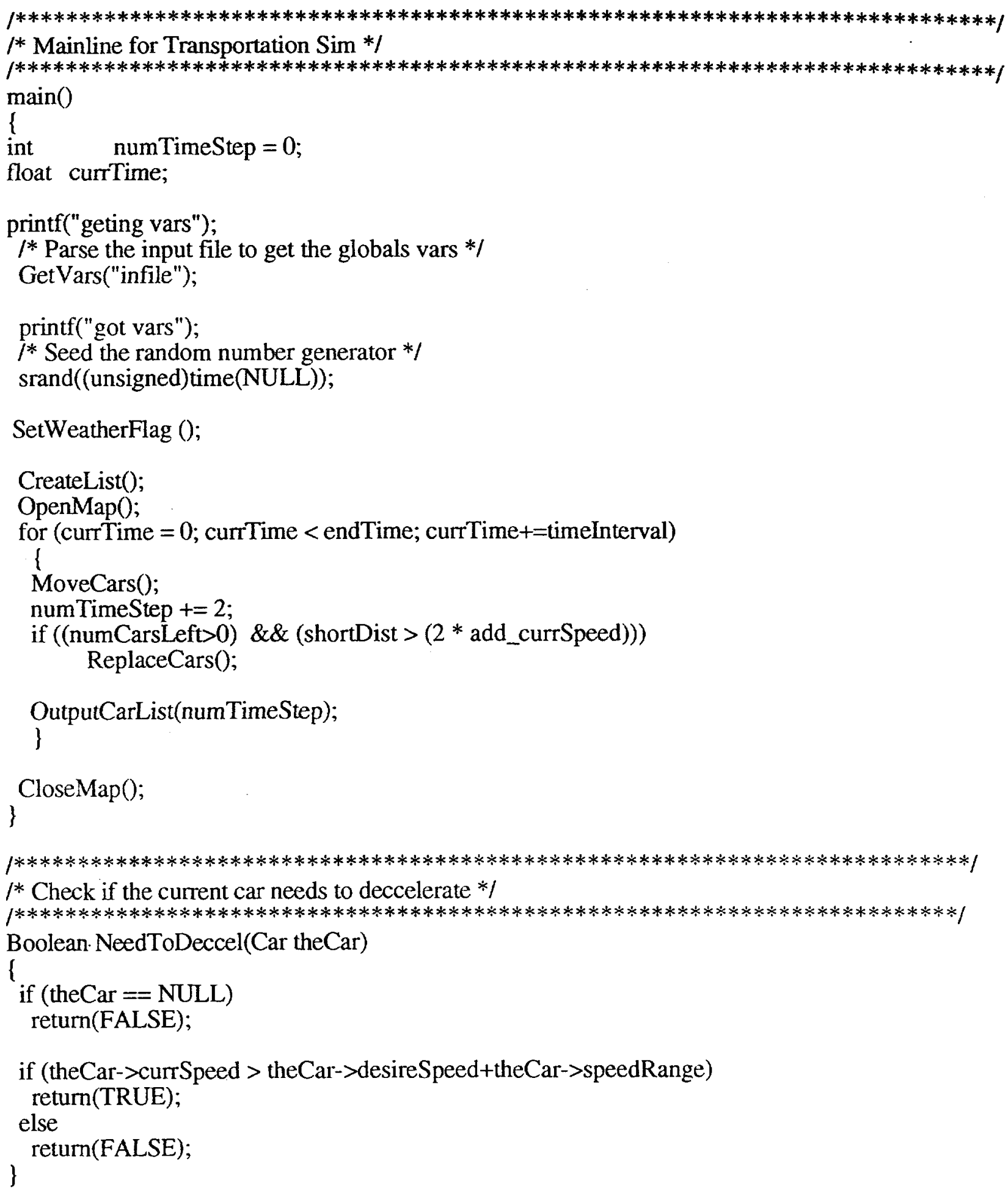




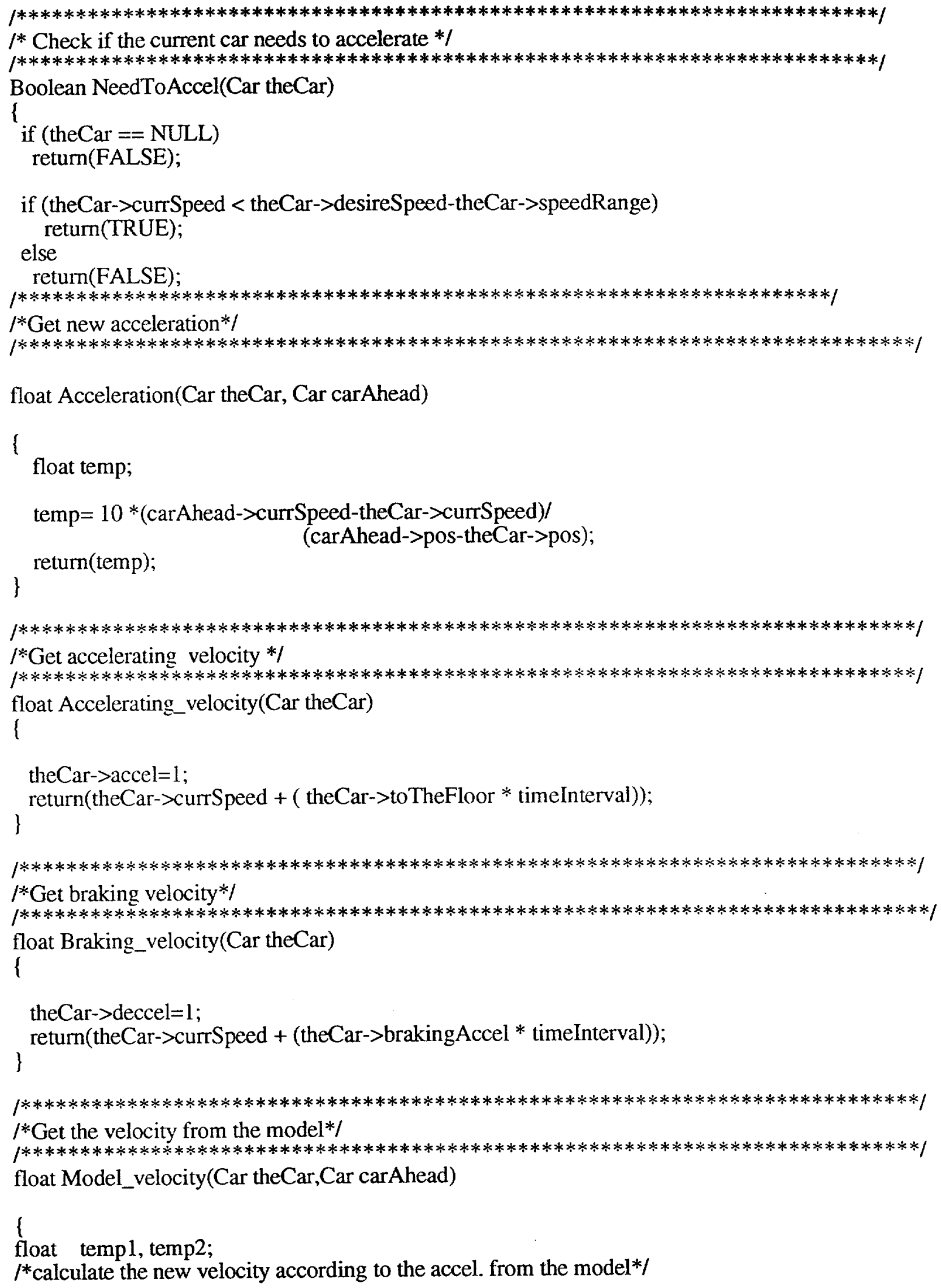


temp $1=$ theCar->currSpeed $+($ Acceleration $($ theCar, carAhead $) *$ timeInterval $)$;

I*Randomize the new speed*I

temp $2=$ (GetRandomRange(temp1-theCar->speedRange, temp1+theCar->speedRange));

\section{/*set color flags*I}

ChangeFlags(theCar, temp2);

return(temp2);

\}

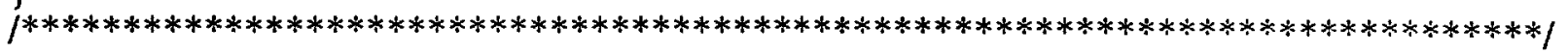

$1 *$ set weather flag */

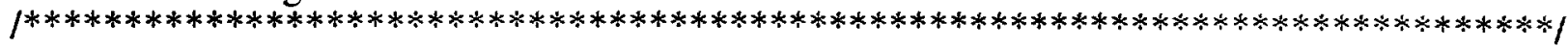

void SetWeatherFlag ()

\{

if $(($ strcmp(weather, "Sunny")) $==0$ )

\{

sunny $=1$;

fog $=$ rain $=0$;

\}

else

\{

if ((strcmp(weather, "Fog")) $==0)$

$\{$ fog $=1$

sunny=rain $=0$;

\}

else

$\{$ rain $=1$;

\}

sunny $=$ fog $=0$;

)

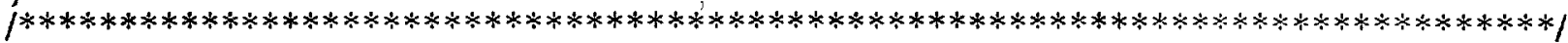

$/ *$ set flags according to change in velocity*/

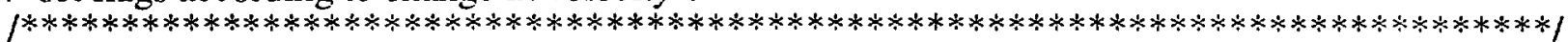

void ChangeFlags(Car theCar, float newVelocity)

\{float temp;

temp $=$ newVelocity - theCar- $>$ currSpeed;

if(temp $>0$ )

theCar->accel $=1$;

else

if (temp <0)

theCar->deccel $=1 ;\}$

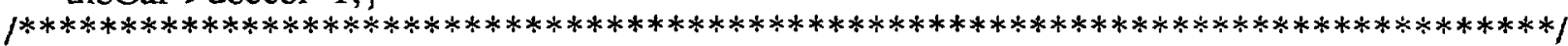

$1 *$ Get new velocity for head car*l

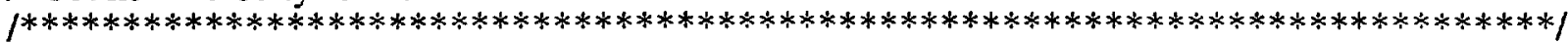

float New_head_velocity(Car theCar)

(float temp;

if (NeedToAccel(theCar)) $\quad l^{*}$ Is the car going too slow? */ else return(Accelerating_velocity(theCar)); $\left.\quad\right|^{*}$ then speed up */ 


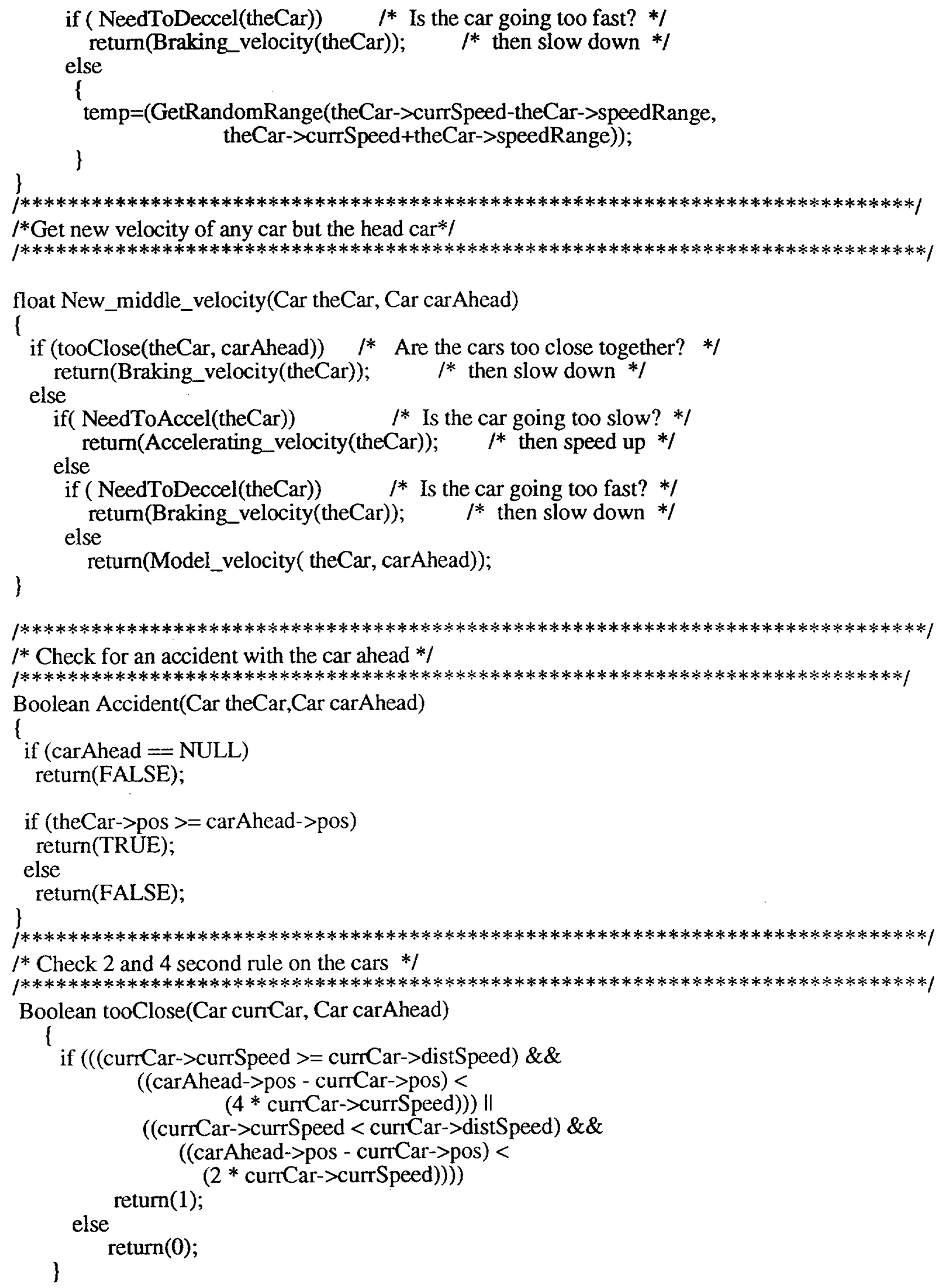




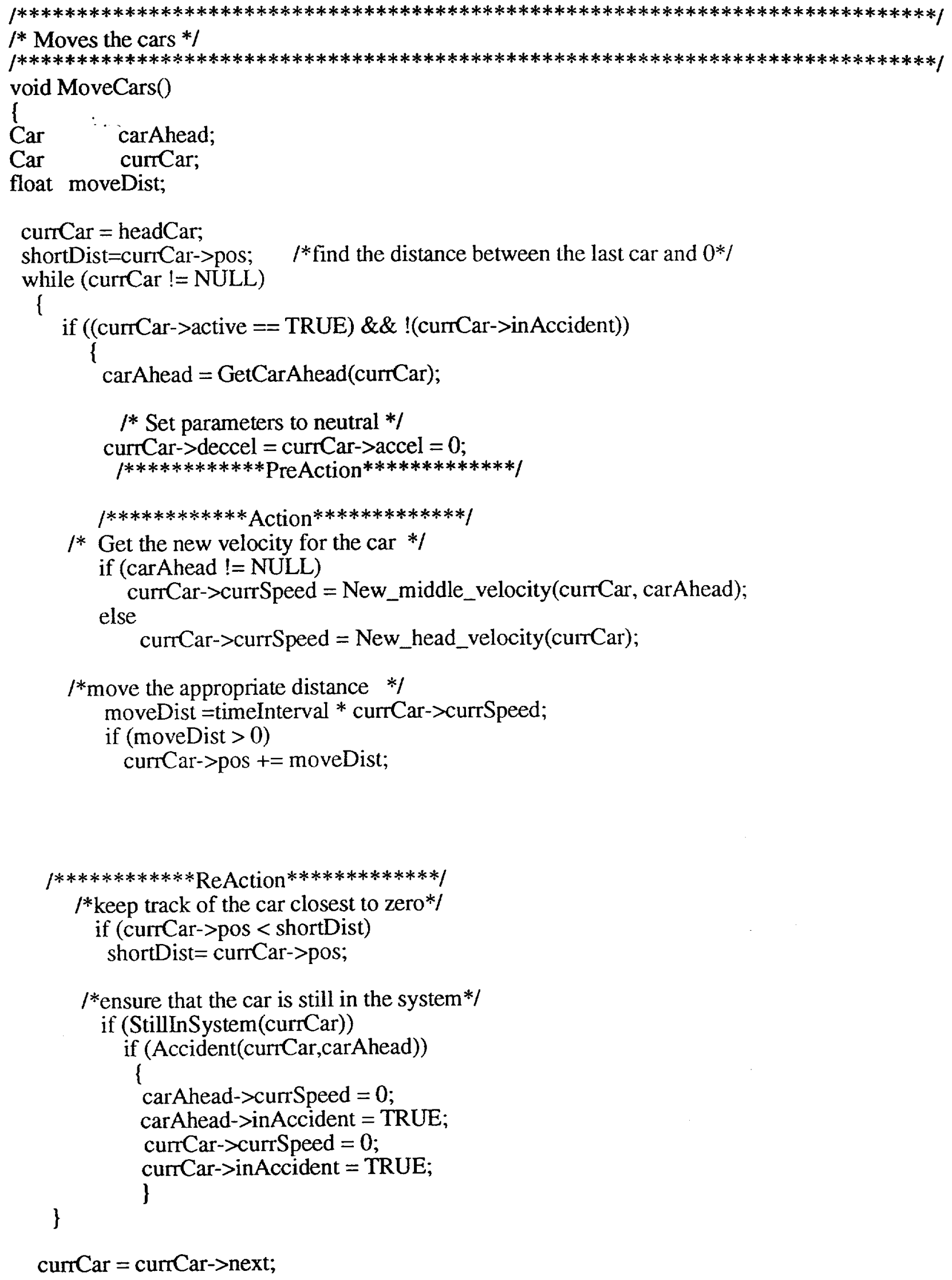

$1 *$ Get the new velocity for the car $* 1$

if (carAhead $!=$ NULL)

currCar->currSpeed $=$ New_middle_velocity $($ currCar, carAhead); else

currCar->currSpeed $=$ New_head_velocity $($ currCar $)$;

$1 *$ move the appropriate distance $* /$

moveDist $=$ timeInterval $*$ currCar- $>$ currSpeed;

if (moveDist >0)

currCar $->$ pos $+=$ moveDist; 


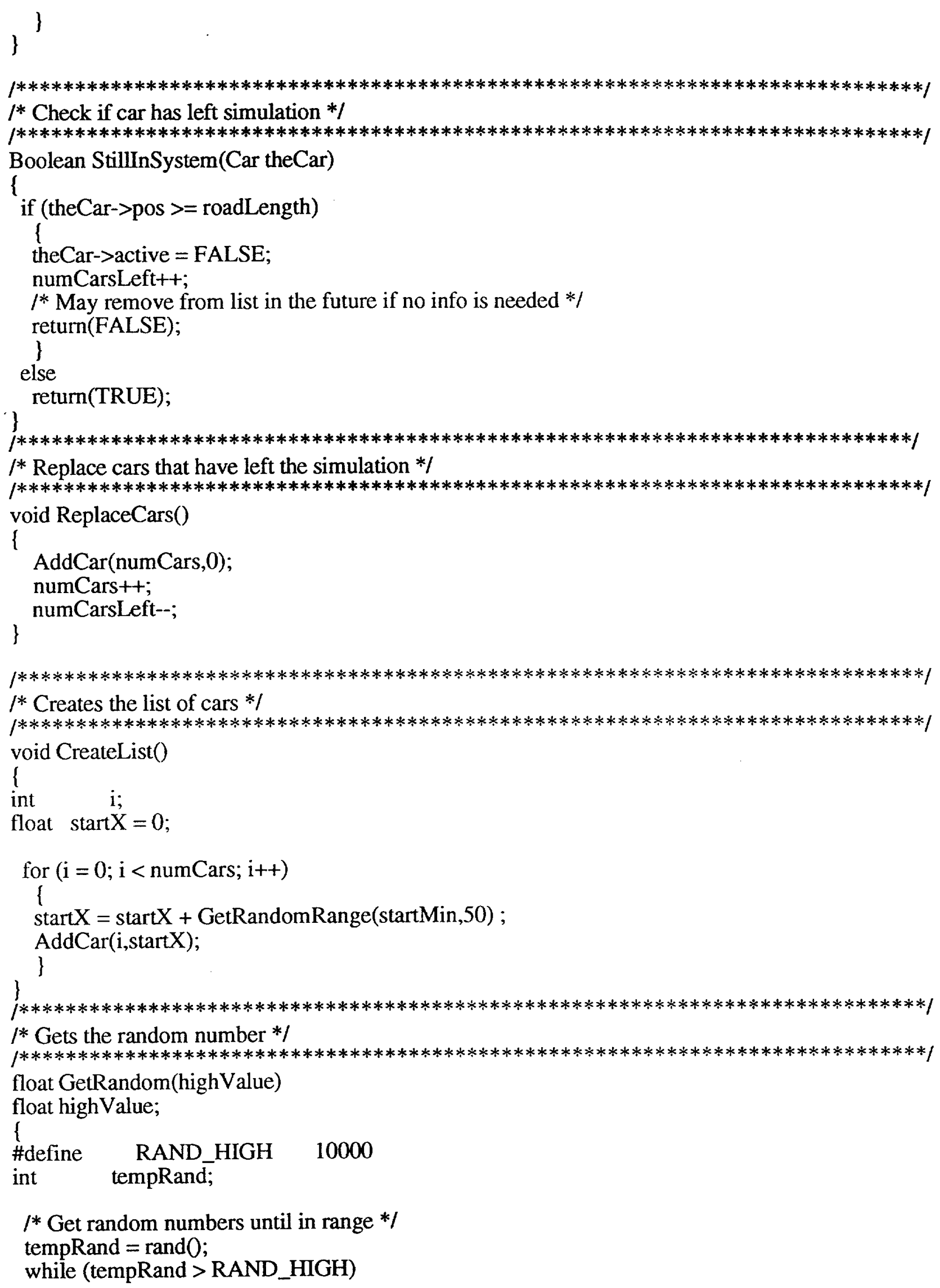

/* Get random numbers until in range */ tempRand $=$ rand 0 ; while (tempRand > RAND_HIGH) 


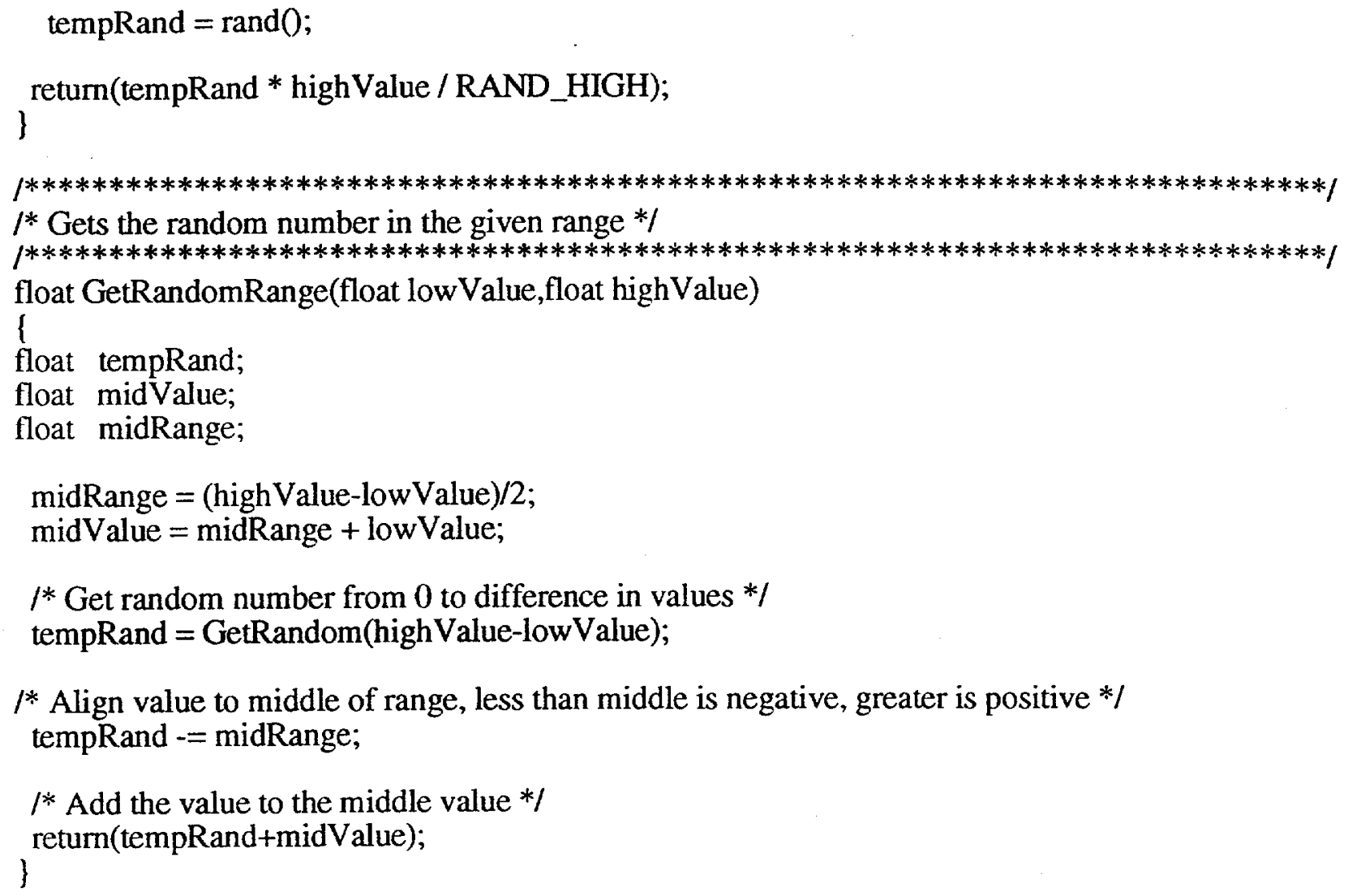

/* Align value to middle of range, less than middle is negative, greater is positive */ tempRand -= midRange;

$I^{*}$ Add the value to the middle value */ return(tempRand+midValue); 
\#include $<$ stdio.h $>$

\section{Carlist.c}

\#include <stdlib.h>

\#include "define.h"

\#include "globals.h"

extern Car headCar;

extern Car selectedCar;

extern int sunny, rain, fog;

void AddCarlmage(int xmarker,int ymarker,int color);

void SetGlobalsVars(Car currCar);

void SetKindOfDriver(Car curCar);

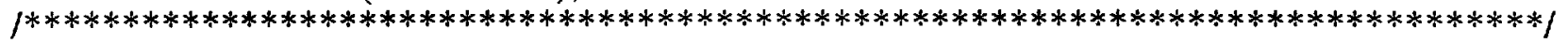

$1 *$ Handles the adding of an atom $* 1$

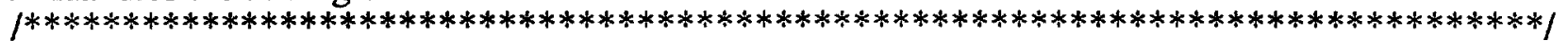

void AddCar(int carNumber,float pos)

I

Car backCar = NULL;

Car $\quad$ currCar = NULL;

if (headCar != NULL)

\{

backCar = headCar;

while (backCar->next ! = NULL)

backCar = backCar->next;

\}

currCar $=($ Car $)$ malloc $($ sizeof $($ struct CarStruct $))$;

if $($ currCar $==$ NULL)

\{

printf("Out of memory in AddCarln");

exit(1);

\}

if (backCar != NULL)

backCar->next = currCar;

if (headCar $==$ NULL)

headCar = currCar;

currCar $->$ carNumber $=$ carNumber;

currCar->active $=$ TRUE;

currCar $->$ deccel $=0$;

currCar->accel $=0$;

currCar->desireSpeed $=$ add_desireSpeed $; * \mathrm{~m} / \mathrm{s} * /$

currCar- $>$ currSpeed $=$ add_currSpeed; $/ * \mathrm{~m} / \mathrm{s} * 1$

currCar->speedRange $=$ add_speedRange; $/ * \mathrm{~m} / \mathrm{s} *$ /

SetKindOfDriver(currCar);

SetGlobalsVars(currCar);

currCar->pos = pos;

currCar->inAccident $=$ FALSE; 


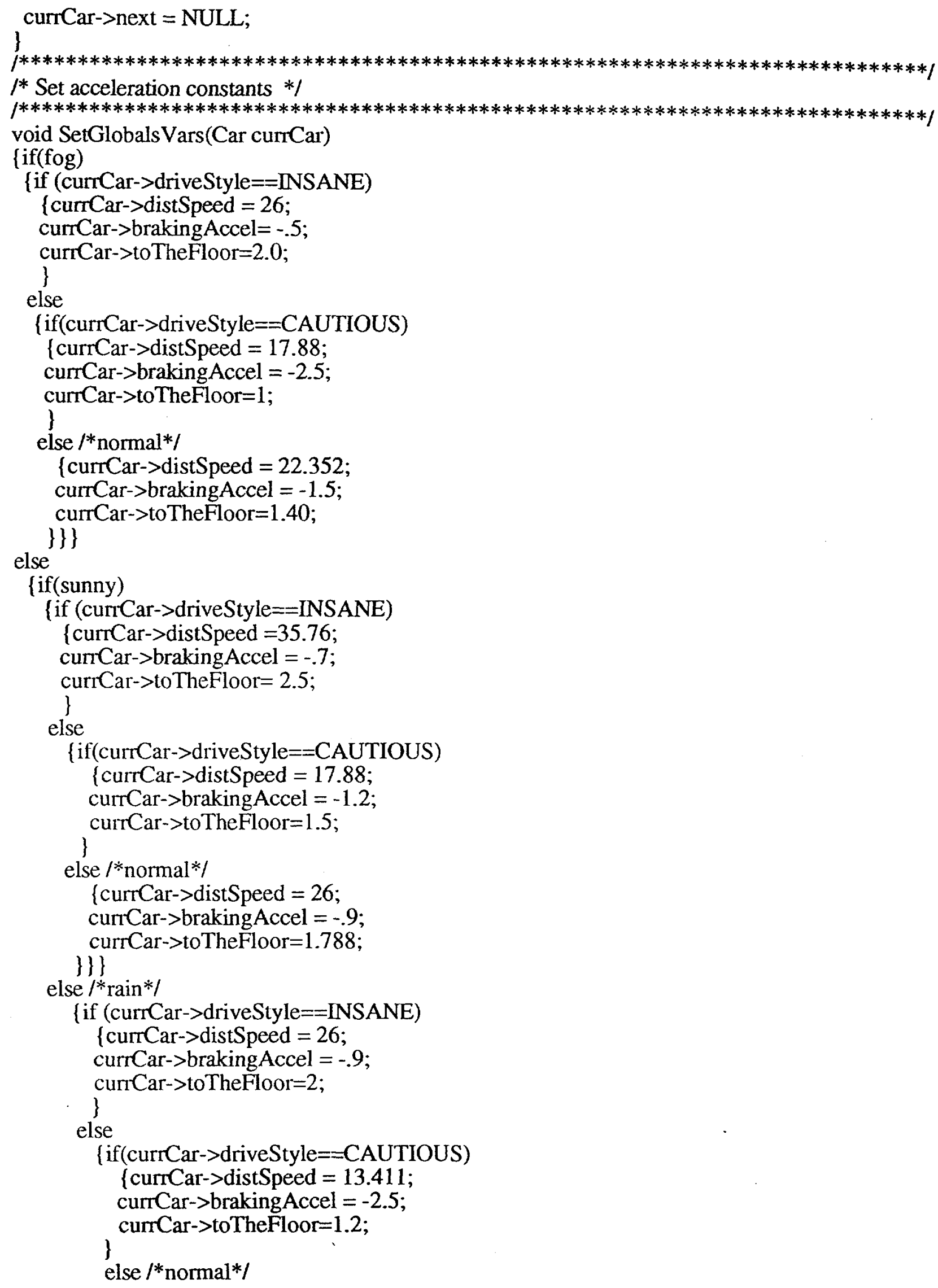


$\{$ currCar->distSpeed $=17.88$

currCar->brakingAccel $=-.9$;

currCar->toTheFloor $=1.5$;

$$
\text { \}\}\}\}\} }
$$

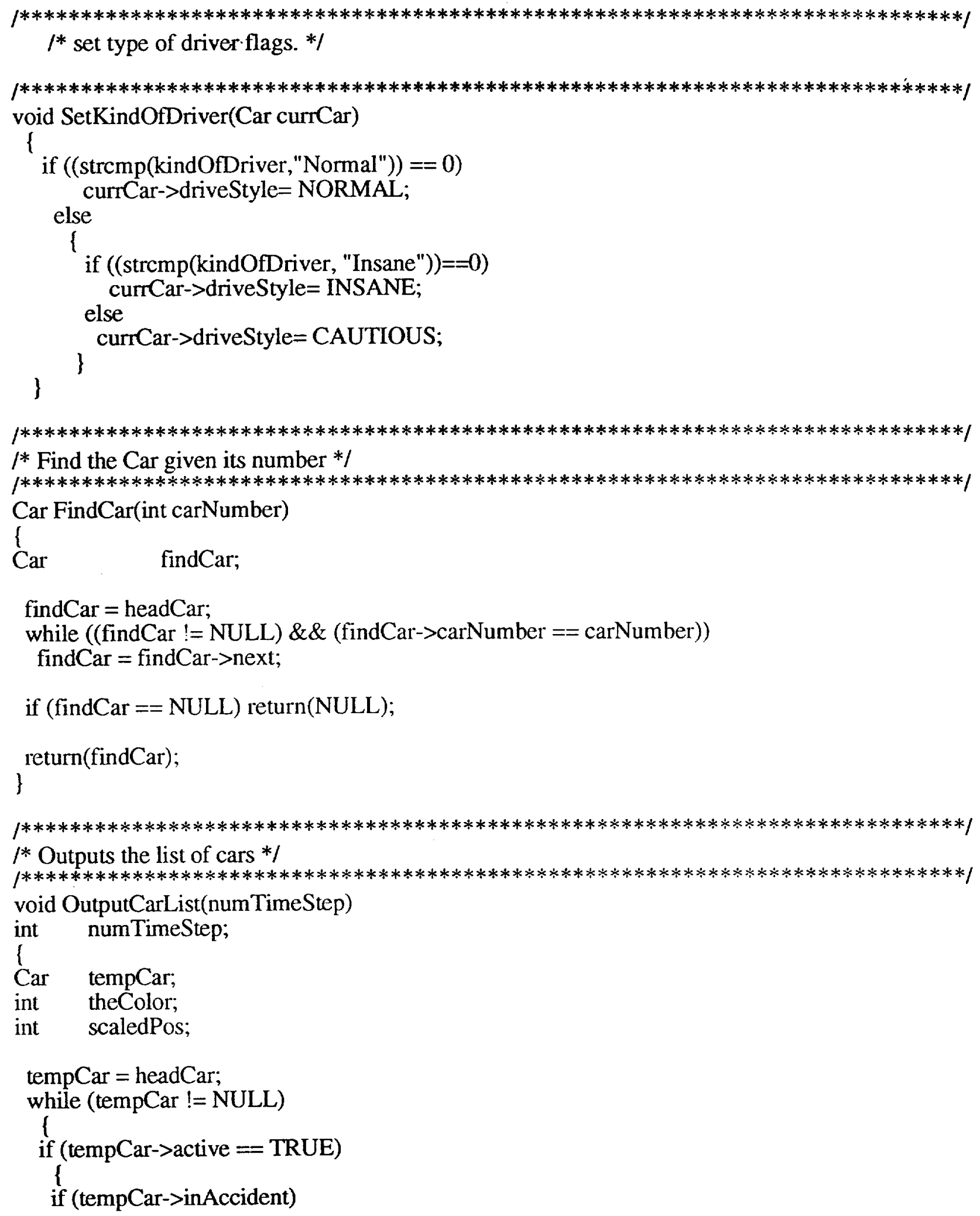




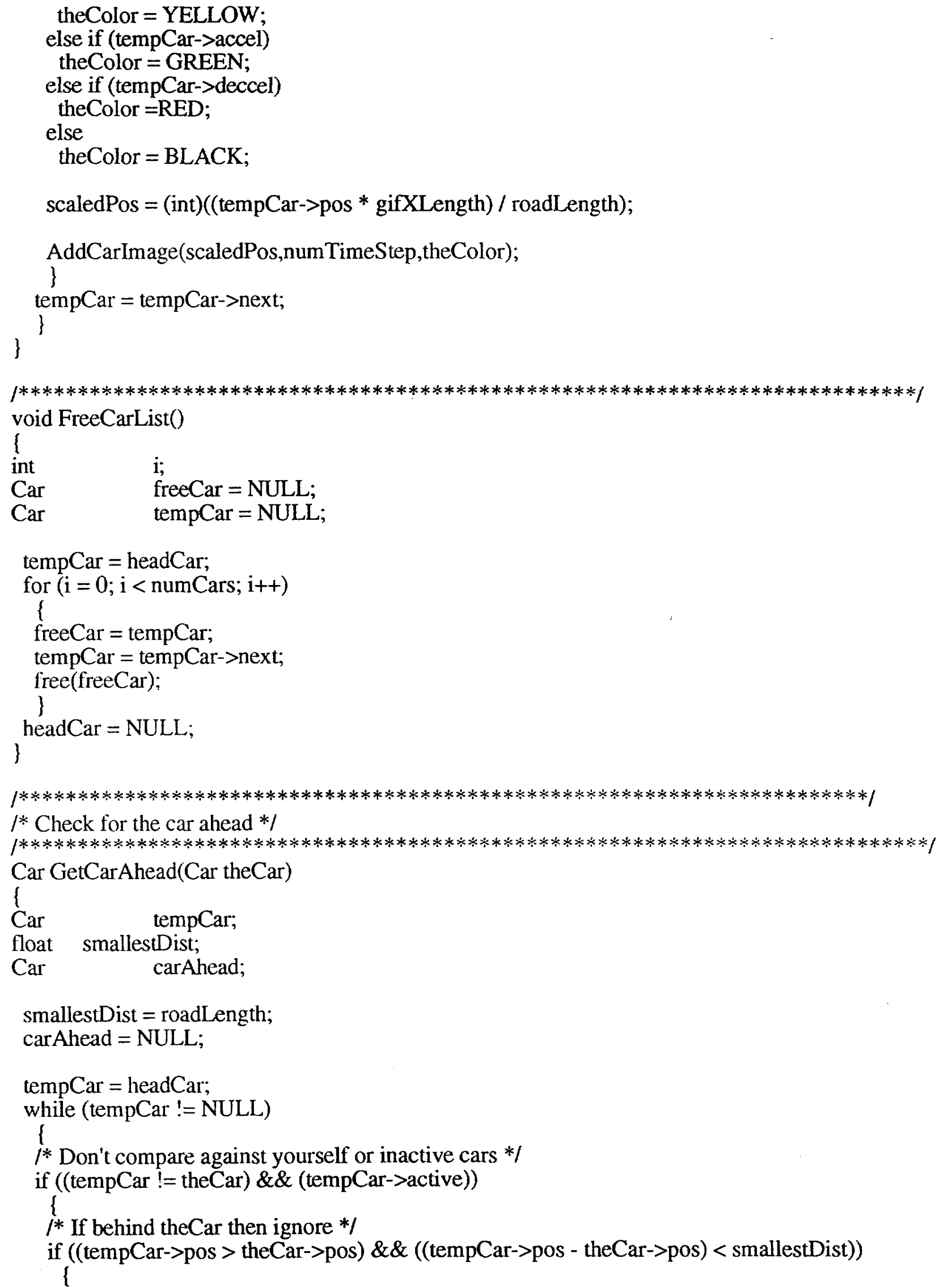




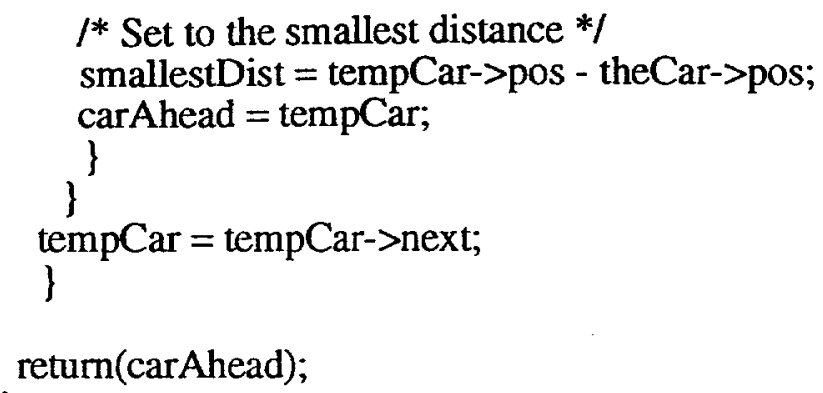




\section{Define.h}

\#define LINELENGTH 80

\#define EOS 10 '

typedef unsigned char Boolean;

\#ifndef TRUE

\#define TRUE 1

\#define FALSE

0

\#endif

/*Define constants for driving styles for easy reading*/

\#define INSANE 1

\#define CAUTIOUS 2

\#define NORMAL 3

/* Define colors in the Apple System Palette *

\#define RED 35

\#defineGREEN 5

\#define YELLOW 225

\#define BLACK 255

/* Car structure *I

typedef struct CarStruct

I

int carNumber;

Boolean active;

int deccel;

int accel;

float desireSpeed;

float currSpeed;

float speedRange;

float distSpeed;

float brakingAccel;

float toTheFloor;

int driveStyle;

Boolean inAccident;

float pos;

struct CarStruct *next;

\} *Car; 


\section{Globals.h}

$\begin{array}{lc}\text { /* Global variables defined in io.c } * / \\ \text { extern int } & \text { numCars; } \\ \text { extern float } & \text { endTime; } \\ \text { extern float } & \text { timeInterval; } \\ \text { extern float } & \text { roadLength; } \\ \text { extern float } & \text { add_desireSpeed; } \\ \text { extern float } & \text { add_currSpeed; } \\ \text { extern float } & \text { add_speedRange; } \\ \text { extern int rain; } & \\ \text { extern int } & \text { sunny; } \\ \text { extern int fog; } & \\ \text { extern char } & \text { weather[6]; } \\ \text { extern char } & \text { kindOfDriver[9]; } \\ & \\ \text { extern char } & \text { outfile[50]; } \\ \text { extern char } & \text { sourcefile[50]; } \\ \text { extern char } & \text { palfile[50]; } \\ \text { extern int } & \text { gifXLength; } \\ \text { extern int } & \text { gifYLength; } \\ \text { extern int } & \text { markerXSize; } \\ \text { extern int } & \text { markerYSize; }\end{array}$




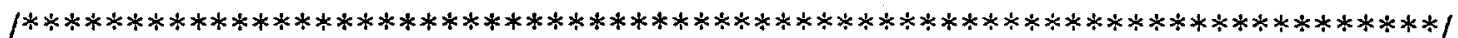

$1 *$ Get variables from the input file $* 1$

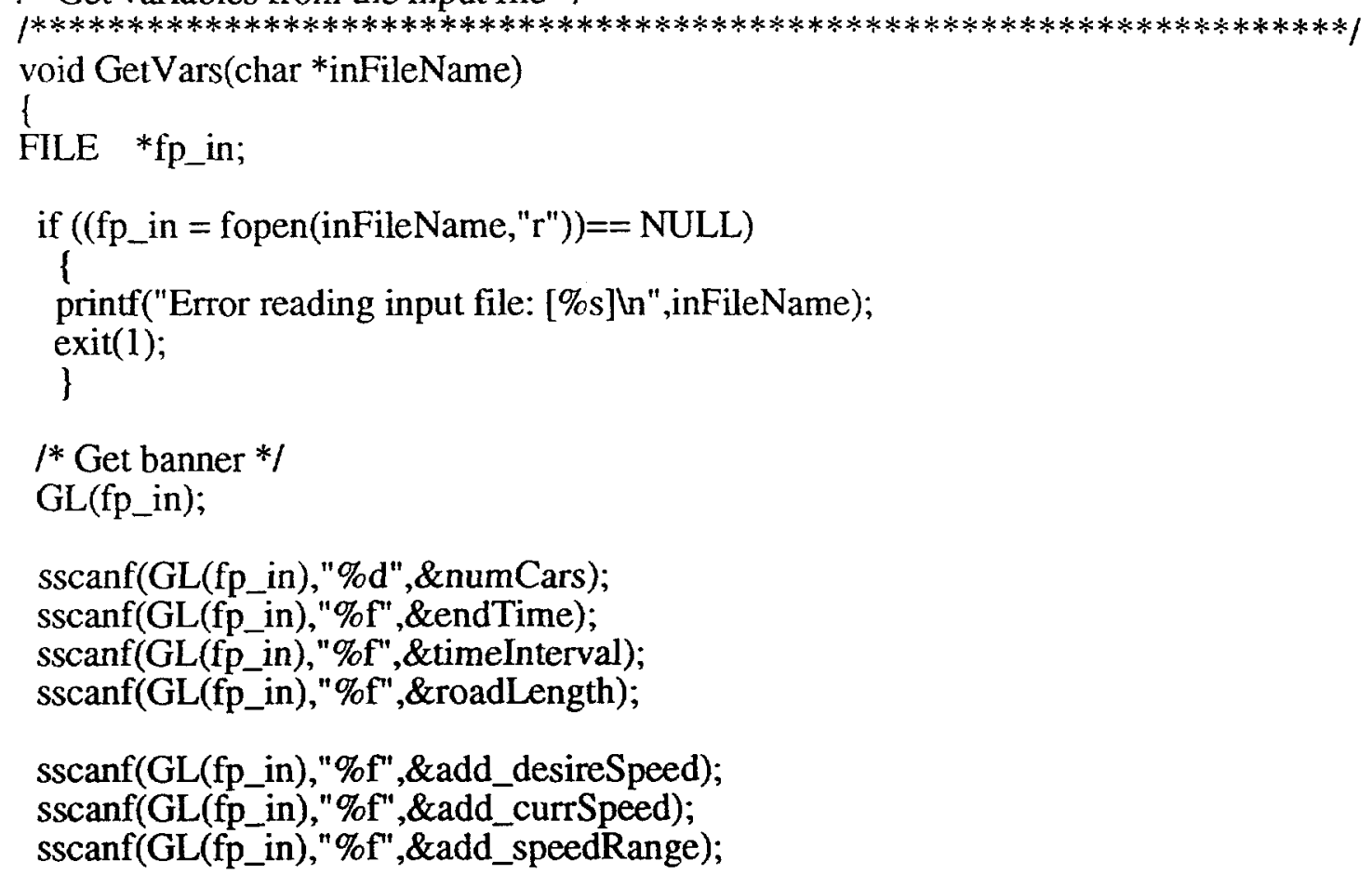


/**************************************************************************/

$l^{*}$ Gets the next line from the input file $* l$

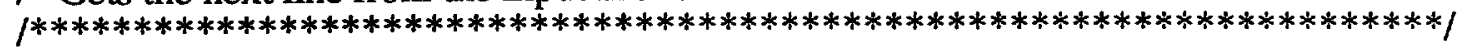
char *GL(FILE *fp)

l fgets(tempString, 80,fp); return(tempString); \} 


\section{Trans_CGI.c}

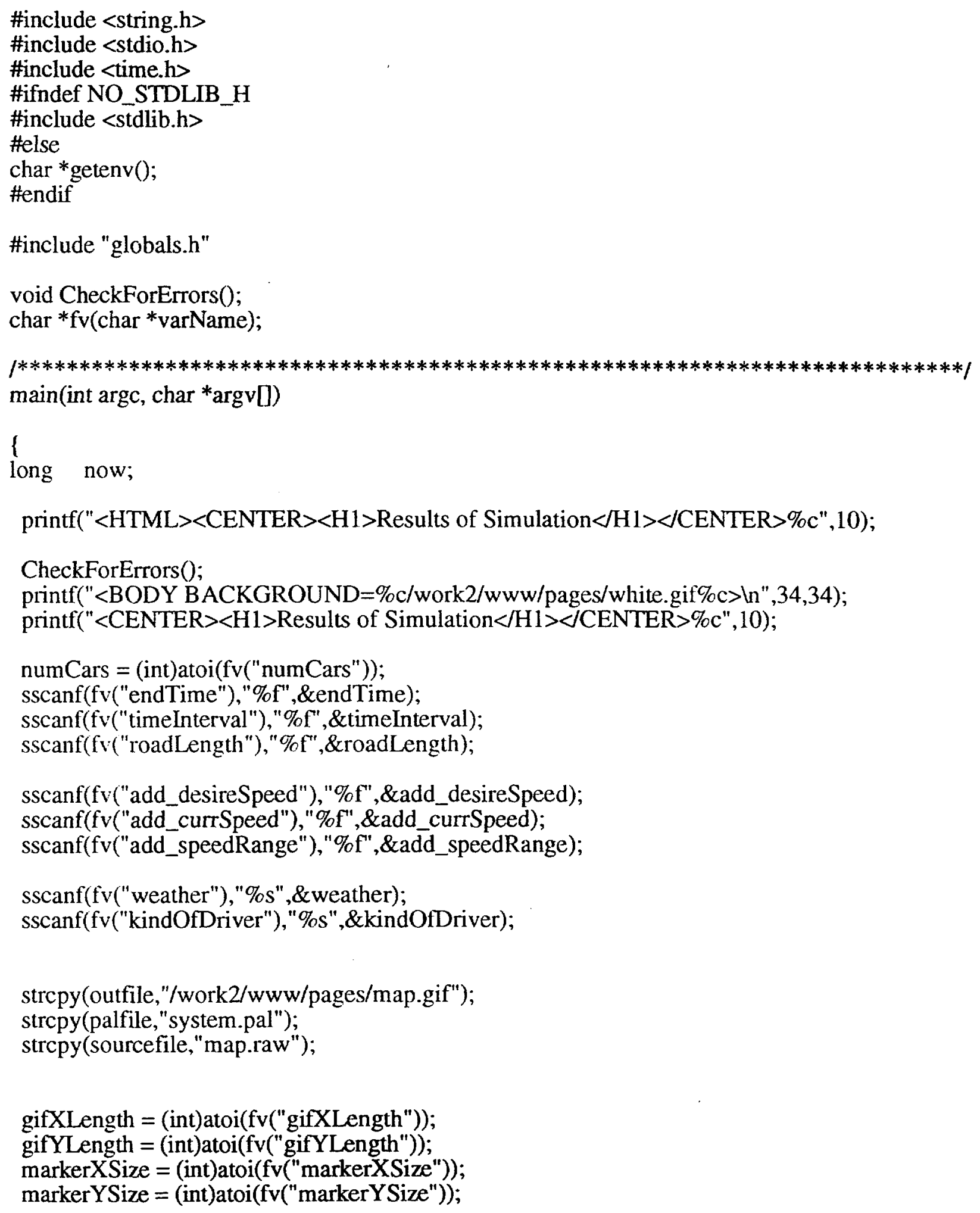




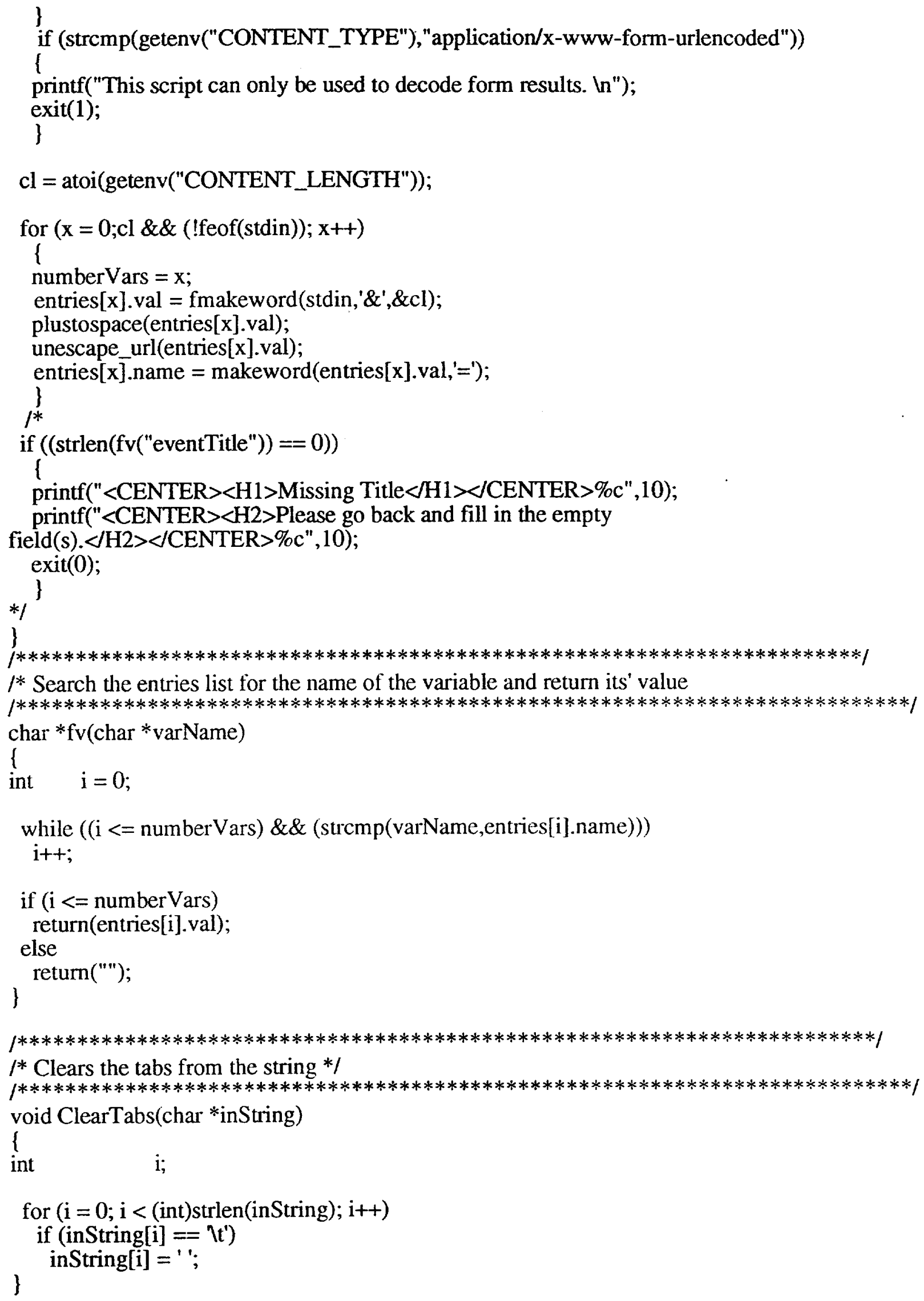




\section{Appendix B: Select HTML Files}

\section{Introduction.html}

$<$ html>

$<$ title>

Traffic Simulation

$<$ title>

<body background="metro.feature1a1.gif">

$<$ center $>$

$<$ b $><$ img src $=$ "welcome.gif " $><$ center $>$

$<\mathrm{hl}><$ center $>$ to the Freeway $<\mathrm{hl}>$

$<\mathrm{b}>$ A Transportation Simulation $<$ b $>$

$<\mathrm{H} 4>$

Joint project with $<$ A HREF="http://www.llnl.gov" $>$ LLNL $</ A>$ 's

$<$ A HREF="http://ep.llnl.gov" $>$ Education Program $\langle/ \mathrm{A}\rangle\langle\mathrm{BR}\rangle$

and <a href="http://www.COSC.gov/SMARTNET.HTML">SMARTNet $<$ a $><B R>$

$</ \mathrm{H} 4>$

$<$ a href="addressing.html" $>$ Addressing $<$ a $>$ the $<$ a href="http://www.nctm.org" $>$ National

Council of Teachers of Mathematics $</ a>(N C T M)<A$

href="http://www.enc.org/online/NCTM/280dtoc1.html" $>$ Curriculum Standards $</$ A $>$ for School

Mathematics $<\mathrm{p}>$

Imagine you are looking through a window at a stretch of freeway. You can only see one particlular section of the road. Cars come into your vision, pass by you, and then go on their way. What we have created is an opportunity to take a closer look at those cars and the math and science that determine their motion. $\langle\mathrm{p}>$

$<$ CENTER $>$

$<\mathrm{h} 2>$ What is there to do here? $<\mathrm{br}></ \mathrm{h} 2>$

$<$ h4 $>$ You could...<br $>$

...skip everything else and $<$ a href $=$ "transnew.html" $>$ jump to the simulation. $<$ a $><$ a

href="presentation $7 . h t m l "><$ img src="cara.gif" $></ a><b r>$

...look at some $<$ a href $=$ "previous.html" $>$ previous simulations. $<$ a $><$ br $>$

...learn $<$ a href $=$ "purpose.html" $>$ why $<$ a $>$ we created these pages. $<$ br $>$

...learn $<$ a href $=$ "whoAreWe.html" $>$ who $<$ /a $>$ we are. $<$ br $>$

...learn $<$ a href $=$ "details.html" $>$ how $<$ la $>$ we made this simulation. $<$ br $>$

$<\mathrm{p}>$

You could also... $<$ br $>$

...learn about $<$ a href $=$ "conversion.html" $>$ converting $</$ a $>$ from one unit of measure to another $<b r>$

...learn about the relationship between position, velocity, acceleration. <br $>$

...learn about $<$ a href $=$ "modeling.html" $>$ simulations and mathematical models $</ a\rangle .<b r\rangle$

...learn about the $<$ a href="carfollowing.html" $>$ car-following model $<$ /a $>$ used in this simulation. $</ \mathrm{h} 4>$

$<\mathrm{h} 2><\mathrm{a}$ href $=$ "foreducators.html" $>$ Resources for Educators $</ \mathrm{a}></ \mathrm{h} 2>$

$<\mathrm{h} 2>$ Why teach science and math by modeling traffic? $<\mathrm{h} 2><\mathrm{p}>$ 
$<$ ul $><$ li $>$ Traffic involves a spectrum of physics and mathematics. Algebra, geometry, probability and calculus can be found between the bumpers of a traveling car.

$<\mathrm{p}\rangle$

$<$ li > Learning takes place when information is presented in an interesting, meaningful way. The motion of a single car and the traffic of a freeway are systems which lend themselves to teaching and are a relevant to virtually every person's life.

$<$ ul $>$

$<$ a href $=$ "presentation6.html" $>$ Way back $<$ a $>$

$<$ body $>$

$<$ html $>$

\section{TransNew.html}

<BODY BACKGROUND="metro.featurela1.gif ">

$<$ TITLE $>$ Transportation Variables Form $<$ TTTLE $>$

$<$ CENTER $><\mathrm{H} 1>$ Transportation Variables Form $</ \mathrm{H} 1></$ CENTER $>$

Please fill in the following information. Press the Run button when finished. $<\mathrm{BR}>$

$<\mathrm{HR}>$

$<$ FORM METHOD="POST" ACTION="http://edprog.llnl.gov/cgi-bin/trans_cgi">

$<$ CENTER $>$

$<\mathrm{B}>$ Simulation Parameters: $<\mathrm{B}><\mathrm{P}>$

$<$ CENTER $>$

$<\mathrm{B}>$ Number of Cars $</ \mathrm{B}><\mathrm{BR}>$

How many cars are on your freeway? The simulation will initially place the number you give or less cars on the road. It will only place as many as will fit without having collision occur before time $=1 .<\mathrm{BR}>$

$<$ CENTER $>$

Number of Cars: $<$ INPUT INPUT SIZE $=5$ NAME="numCars" VALUE $=" 10 "><P>$ $<$ CENTER $>$

$<\mathrm{B}>$ Ending Time in Seconds $</ \mathrm{B}><\mathrm{BR}>$

How long do you want to watch? Give the total length of time for the simulation to run. Time should be measured in seconds. $\langle\mathrm{BR}\rangle$

$<$ CENTER $>$

Ending Time in seconds: $<$ INPUT INPUT SIZE $=10$ NAME="endTime" VALUE $=" 80.0 "><P>$

$<$ CENTER $>$

$<\mathrm{B}>$ Time Interval $<\mathrm{B}><\mathrm{BR}>$

The simulation works by calculating positions, velocities and accelerations at different times. Time interval is the amount of time between these calculations. Give a time interval measured in

seconds. $<$ BR $>$

$<$ CENTER $>$

Time interval in seconds: $<$ INPUT INPUT SIZE $=10$ NAME $="$ timeInterval" VALUE $=" 1.0^{\prime \prime}><P>$ $</$ CENTER $>$

$<\mathrm{B}>$ Road Length $<\mathrm{B}\rangle\langle\mathrm{BR}\rangle$

You are viewing only one section of the freeway. How long is it? Give the length of the road in meters.<BR>

$<$ CENTER $>$

Road Length in meters: $<$ INPUT INPUT SIZE $=10$ NAME $=$ "roadLength" VALUE="400.0" $><P>$ $<$ CENTER $>$ 
$<\mathrm{B}>$ Desired Speed $<\mathrm{B}><\mathrm{BR}>$

As people drive, they usually have a desired speed in mind. Sometimes things prevent them from driving at that speed, i.e. a traffic jam, an accident. Give the desired speed for your cars. Desired speed is measured in meters per second $(\mathrm{m} / \mathrm{s})$. Do you need to $<\mathrm{a}$

href $=$ "conversion.html" $>$ convert $</ a>$ your desired speed from miles per hour to meters per second? $\langle\mathrm{BR}>$

$\langle$ CENTER $>$

Desired speed in $\mathrm{m} / \mathrm{s}:<\mathrm{INPUT}$ INPUT SIZE=10 NAME="add_desireSpeed"

VALUE $=" 2.7 "><\mathrm{P}\rangle$

$<$ CENTER $>$

$<\mathrm{B}>$ Current Speed $<\mathrm{B}><\mathrm{BR}>$

The cars on your freeway could be moving when they come into view. What is their speed when you first see them? Give current speed in meters per second; don't forget to $<a$

href="conversion.html" $>$ convert $<$ a $>$ if you need to. $<\mathrm{BR}>$

$<$ CENTER>

Current speed in $\mathrm{m} / \mathrm{s}:<$ INPUT INPUT SIZE=10 NAME="add_currSpeed" VALUE="2.7" $><\mathrm{P}>$ $<$ CENTER $>$

$<\mathrm{B}>$ Range of Speed $<\mathrm{B}>$

$<\mathrm{BR}>\mathrm{Few}$ drivers maintain the exact same speed for any length of time. Speed is constantly being adjusted up or down a little. Choose a range of speed you would like your cars to stay within.

This range is measured in meters per second.<BR $>$

$<$ CENTER>

Range of speed in m/s: <INPUT INPUT SIZE=10 NAME="add_speedRange"

VALUE $=" 0.5 "><\mathrm{P}>$

$<$ CENTER $>$

$<\mathrm{B}>$ Weather $<$ B $><\mathrm{BR}>$

The weather conditions effect cars traveling on the freeway. What are the weather conditions for your freeway? What kind of weather are you driving in? $<B R>$

$<$ CENTER $>$ Weather (Choose one):

$<$ SELECT NAME = "weather" $>$

$<$ OPTION SELECTED > Sunny

$<$ OPTION $>$ Fog

$<$ OPTION $>$ Rain

$<$ SELECT $><$ P $>$

$</$ CENTER $>$

$<\mathrm{B}>$ Driving Style $<\mathrm{B}><\mathrm{BR}>$

Every one has their own driving style. Some people cruise casually while other are constantly slamming on the breaks or zooming along inches from the other cars. What kind of driving style do your cars have? $<\mathrm{BR}>$

$<$ CENTER>

What kind of driver are you?

$<$ SELECT NAME="kindOfDriver">

$<$ OPTION S ELECTED> Normal

$<$ OPTION $>$ Insane

$<$ OPTION $>$ Cautious

$<$ Select $><\mathrm{P}\rangle$

$<$ CENTER $>$

$<\mathrm{B}>$ The Picture $<\mathrm{B}><\mathrm{BR}>$

How big do you want your picture to be? Choose dimensions for your picture. The dimensions will be measured in pixels. (Pixels are really small.) $<B R>$

$<$ CENTER $>$ 
Width of output in pixels: <INPUT INPUT SIZE=10 NAME="gifXLength"

VALUE $=" 400 ">\langle B R>$

Height of output in pixels: <INPUT INPUT SIZE=10 NAME="gifYLength"

VALUE $=" 200 ">\langle\mathrm{P}\rangle$

$<$ CENTER>

$<\mathrm{B}>$ The Cars $<\mathrm{B}><\mathrm{BR}>$

The cars will be displayed as dots of pixels. What is the dimension of your car in pixels? $<\mathrm{BR}>$ $<$ CENTER $>$

Width of car in pixels: $<$ INPUT INPUT SIZE=10 NAME="markerXSize" VALUE="3" $><B R>$ Heigth of car in pixels: <INPUT INPUT SIZE=10 NAME="markerYSize" VALUE="2" $><P>$

It is time to run your simulation. Click the button below. $<\mathrm{BR}>$

$\langle\mathrm{P}\rangle\langle$ Input type=submit VALUE=" Run the simulation " $><\mathrm{P}\rangle$

<INPUT TYPE="reset" VALUE="Clear and reset">

$<$ FORM $>$

$<\mathrm{P}><\mathrm{P}><$ FONT $>$ For more information contact Brian Lindow, lindow@llnl.gov $<\mathrm{BR}>$

$<$ A HREF="http://www.llnl.gov/disclaimer.html">

$<$ IMG BORDER=0 align=middle src="http://www.llnl.gov/lnl/GIFs/lll.gif">

LLNL Disclaimers $<$ A $><$ BR $>$ UCRL-MI-122360 $<$ BR $>$

$<$ CENTER $><$ img src="1_hand.gif" alt="Picture of hand pointing back" $><\mathrm{A}$ href $=$ "

introducion.html " $>$ Back to the introduction $</ \mathrm{A}><$ address $>$ Kimberly J. Drake $<$ br $>$

Drake5@1lnl.gov $<$ address $>$

\section{CarFollowing.html}

$<$ html $><$ head $><$ title $>$ car-following $<$ title $><$ head $>$

$<$ body background=" metro.feature la l.gif" $>$

$<$ hl $><$ center $>$ Car-Following?...What? $</$ center $><$ h $1><$ P $>$

The simulation uses several different methods to determine the new acceleration when calculating the new velocity of a car. It asks the questions...

$<\mathrm{ul}\rangle$

$<$ LI $>$ Is the current car about to crash into the car in front of it?

$<$ LI $>$ Is the car moving too fast?

$<$ LI $>$ Is the car moving too slow?

$<\mathrm{LI}>$ Is the car moving at its desired speed.

$\langle\mathrm{ul}\rangle$

If the car is moving within the given speed range of its desired speed then its new acceleration is calculated according to a car-following model. $<\mathrm{p}>$

$<$ img src ="carbar.jpeg" $><\mathrm{P}>$

$<b>$ Car-following $<$ b $>$ is based on the idea that any car following another car on a single lane road will drive according to the car ahead of it. $\langle\mathrm{P}\rangle$ For example, if the first car slows down then the second car slows down. If the first car stops, then the second car stops. If the first car speeds up then the second car has the opportunity to speed up. $<\mathrm{P}>$

This car-following model uses $\mathrm{a}<\mathrm{b}>$ scaled ratio $<$ b $>$ of the $<\mathrm{b}>$ relative velocities $<\mathrm{b}>$ of the cars to the $\langle\mathrm{b}>$ relative positions $<\mathrm{b}>$ of the cars to calculate the new $\langle\mathrm{b}>$ accelration $<$ b $>$ of the second car. $<$ br $>$

$<$ h3 $>$ Relative Position $</$ h3 $>$

Relative position refers to the cars' locations relative to each other. We don't care where the cars are in space, what we care about is the $\langle\mathrm{b}>$ distance $\langle/ \mathrm{b}>$ between them. We calculate the cars' 
relative positions by subtracting the position of the second car from the position of the first car, in other words, $<\mathrm{p}><$ center $>$ Relative Position = Position Car $1-$ Position Car $2</$ center $><\mathrm{p}\rangle$ $<$ h3 $>$ Relative Velocity $</ \mathrm{h} 3><$ br $>$

Relative velocity refers to the cars velocities relative to each other. We don't care how fast they cars are moving. We care about the $\langle\mathrm{b}>$ difference $</ \mathrm{b}\rangle$ in their speeds. To find relative velocity, we subtract the velocity of the second car from the velocity of the first. In other words, $<p>$

$<$ center $>$ Relative Velocity = Velocity Car 1 - Velocity Car $2<$ center $><$ br $>$

$<\mathrm{h} 3>$ Scaled Ratio $<\mathrm{h} 3>$

The model we used puts the relative velocity over the relative position. Think about that for a moment. In algebra, it would look like: $\langle$ br $>$

$<$ center $><$ img src $=$ "modell.jpeg" $><$ center $><$ br $>$

Most of the time the ratio is small. (Why is that?) The ratio is scaled by multiplying it by 10 . The final equation looks like this: $\langle\mathrm{p}>$

$<$ center $><$ img src $=$ "model2.jpeg" $><\mathrm{p}></$ center $>$

$<$ center $>$ <img src $=$ "carbar2.jpeg" $><\mathrm{P}></$ center $>$

$<\mathrm{H} 2>$ Things to Do and Think About $</ \mathrm{h} 2>$

$<\mathrm{ol}>$

$<$ li $>$ Find the acceleration of car number 2 using the model and the data below. $<$ br $>$

$<\mathrm{ul}>$

$<$ LI $>$ Velocity $1=25 \mathrm{~m} / \mathrm{s}$

$<$ LI $>$ Velocity $2=24 \mathrm{~m} / \mathrm{s}$

$<$ LI $>$ Position $1=15 \mathrm{~m}$

$<$ LI $>$ Position $2=10 \mathrm{~m}$

$<$ ul $>$

$<\mathrm{LI}>$ What would cause the acceleration of car 2 to be really big? Give an example.

$<$ LI $>$ What would cause it to be really small? Give an example.

$<\mathrm{LI}>$ What would cause the acceleration to be negative? Positive?

$<\mathrm{OL}>$

$<$ <img src="eye_bar.gif " alt="Just a cute little bar. You didn't miss anything!">

$<$ img $\mathrm{src}=$ " 1 hand.gif" alt $=$ "Picture of hand pointing back" $><\mathrm{A}$ href=" introducion.html " $>$ Back to the introduction $</ \mathrm{A}><$ address $>$ Kimberly J. Drake $<\mathrm{br}>$

Drake5@ llnl.gov $<$ /address $>$ 


\title{
Development of $59 \mathrm{Ni}$ Accelerator Mass Spectrometry Capability and Progress Towards Biomedical Tracer Applications. *
}

\author{
aMy Dunlop \\ Harvard University \\ Lawrence Livermore National Laboratory \\ Livermore, California 94550 \\ 9 May 1995
}

Prepared in partial fulfillment of the requirements of the Science and Engineering Reserach Semester under the direction of Jeff McAninch, Research Mentor, in the Lawrence Livermore National Laboratory.

*This research was supported in part by an appointment to the U.S. Department of Energy Science and Engineering Reserach Semester program administered by LLNL under Contract W-7405-Eng-48 with Lawrence Livermore National Laboratory. 


\begin{abstract}
Expanding on previous $63 \mathrm{Ni}$ accelerator mass spectrometry (AMS) research, we have developed AMS capability to detect ${ }^{59} \mathrm{Ni}$ with a detection limit of $\pm 3 \times 10^{8}$ atoms. Included in our methods are a nickel purification step involving the generation of nickel carbonyl and quantification of nickel atoms with an $x$-ray detector. . At present, we are developing protocols for the quantitative extraction of nickel from the tissues of rats that have been dosed with known amounts of $59 \mathrm{Ni}$.
\end{abstract}

\title{
Introduction.
}

Exposure to elevated levels of nickel can lead to cancer and other adverse health conditions. Nickel is also a catalyst in certain biochemical pathways. Accelerator mass spectrometry (AMS) is an ultrasensitive measurement technique for detecting rare, long-lived radioisotopes. ${ }^{1}$

Because ${ }^{59} \mathrm{Ni}$ is rare in nature, has a long half-life $\left(t_{1 / 2} \sim 100000 \mathrm{y}\right)$ and is able to be separated from its cobalt isobar, ${ }^{59} \mathrm{Ni}$ AMS coupled with $\mathrm{x}$-ray detection of nickel atoms ${ }^{2}$ is a possible alternative to the current nickel tracing method, liquid scintillation counting $(\mathrm{LSC})$ of $63 \mathrm{Ni}\left(\mathrm{t}_{1 / 2}=\right.$ 100y). 59Ni AMS would be a preferred alternative for the purpose of biomedical tracing, because the low activity levels ( 1000 less activity than $63 \mathrm{Ni}$ LSC) and sensitivity of measurements imply the ability to examine the effects of lower, more biologically relevant doses of nickel without significant exposure to radioactivity.

The preparation of samples is an important part of Ni AMS. Figure I describes the Ni AMS process and instrumentation. In the end, an x-ray detector is used to count the number of atoms making it through the system. Though we calibrate the instrument for ${ }^{59} \mathrm{Ni}, \mathrm{x}$-rays are measured from both ${ }^{59} \mathrm{Ni}$ and its stable isobar, ${ }^{59} \mathrm{Co}$. Because these spectra overlap, subtraction of the $\mathrm{Co}$ tail from the Ni peak gives the corrected measure of the number of $\mathrm{Ni}$ atoms detected. The subtraction will work only if the Co level in the sample are not extremely higher than the amount of $\mathrm{Ni}$. For this reason, we purify the nickel using a $\mathrm{Ni}(\mathrm{CO}) 4$ process adapted from a method described by Sturgeon, et al. 3 
We have successfully measured the $59 \mathrm{Ni} / \mathrm{Ni}$ ratios of prepared standard solutions with AMS. We have begun dosing animals with both ${ }^{59} \mathrm{Ni}$ and ${ }^{63} \mathrm{Ni}$ in order that comparative measurements of $\mathrm{Ni}$ can be made between ${ }^{59} \mathrm{Ni} \mathrm{AMS},{ }^{63} \mathrm{Ni}$ and ${ }^{63} \mathrm{Ni}$ LSC. Eventually, experiments will be conducted without ${ }^{63} \mathrm{Ni}$ in the dosing solution, reducing the activity of the solution by a factor of about 1000 .

The methods we use for preparing ${ }^{59}$ Ni AMS samples, the results from our AMS detection of ${ }^{59} \mathrm{Ni} / \mathrm{Ni}$ standards and the work we have done concerning the measurement of ${ }^{59} \mathrm{Ni}$ in biological material are discussed in this paper.

\section{Experimental.}

Dilutions. A ${ }^{59} \mathrm{Ni} / \mathrm{Ni}$ standard series was prepared by successive diltuion of a calibrated ${ }^{59} \mathrm{Ni}$ solution in a $1000 \mu \mathrm{g} / \mathrm{ml}$ standard Ni solution used for atomic absorption measurements (VWR). The standard series contains ${ }^{59} \mathrm{Ni} / \mathrm{Ni}$ ratios of $1 \times 10^{-5}, 1 \times 10^{-7}, 1 \times 10^{-8}, 1 \times 10^{-9}$ and $1 \times 10^{-10}$.

AMS sample preparation. Sample preparation was identical for nat $\mathrm{Ni},{ }^{59} \mathrm{Ni}$ and ${ }^{63} \mathrm{Ni}$ measurements. Figure 2 shows the apparatus used for sample preparation. $1 \mathrm{mg} \mathrm{Ni}$ ( $1 \mathrm{mg} \mathrm{Ni} /$ $1 \mathrm{ml} \mathrm{WWR} \mathrm{Ni} \mathrm{Atomic} \mathrm{Absorption} \mathrm{standard} \mathrm{solution)} \mathrm{was} \mathrm{added} \mathrm{with} 9 \mathrm{ml}$ deionized water in a polystyrene test tube. Concentrated $\mathrm{NH}_{4} \mathrm{OH}$ was added dropwise until the $\mathrm{pH}$ of the solution reached 10. The Ni solution was then transferred to the reactor vessel and a carrier gas flow started $(\mathrm{He}, 80 \mathrm{ml} / \mathrm{min})$. After 1 minute, an $80 \mathrm{ml} / \mathrm{min} \mathrm{CO}$ gas flow was added. Following saturation of the nickel solution with CO molecules (approximately 1 minute), $5 \mathrm{ml} 0.5 \%(\mathrm{~m} / \mathrm{v})$ $\mathrm{NaBH}_{4}$ ( $\mathrm{pH}$ raised to 10 with the addition of concentrated $\mathrm{NH}_{4} \mathrm{OH}$ ) was added dropwise $\left(1 \mathrm{ml} / \mathrm{min}\right.$ ) into the reactor vessel to reduce the $\mathrm{Nill}$ to $\mathrm{Ni}^{0}$ for reaction with the $\mathrm{CO}$. After $\mathrm{Ni}(\mathrm{CO})_{4}$ formation, the $\mathrm{CO}$ flow was stopped and the $\mathrm{He}$ flow reduced to $40 \mathrm{ml} / \mathrm{min}$. At this stage, the $\mathrm{Ni}(\mathrm{CO})_{4}$ was condensed on glass beads in a glass trap submersed in a liquid $\mathrm{N}_{2}$ bath. The beads were treated with dimethyldichlorosilane ( $10 \%$ in methanol) to prevent 
chemical bonding of the $\mathrm{Ni}(\mathrm{CO})_{4}$. After inserting the glass pipette end of the apparatus into the sample holder and allowing it to reach approximately $200^{\circ} \mathrm{C}$, the liquid $\mathrm{N}_{2}$ bath was removed. At this time, $\mathrm{Ni}(\mathrm{CO})_{4}$ vaporized and was carried by the He flow into the sample holder where the heat decomposed it to metallic nickel.

\section{Characterization of dosing solutions.}

Total Ni. Total Ni concentrations were measured spectrophotometrically. Aliquots of up to $100 \mu \mathrm{g}$ of Ni was complexed with $1 \mathrm{ml} 5 \%$ DMG in ethanol, $2 \mathrm{ml}$ bromine water and $5 \mathrm{ml}$ concentrated $\mathrm{NH}_{4} \mathrm{OH}$, diluted to $50 \mathrm{ml}$ with deionized water and read at $445 \mathrm{~nm}$.

${ }^{63} \mathrm{Ni}$ activity. $63 \mathrm{Ni}$ activity of the dosing solution was measured with LSC .

Animal experiment protocol- in development. $200 \mathrm{~g}$ female Wistar rats are gavaged with $500 \mu \mathrm{L}$ doses of either $59,63 \mathrm{NiCl}_{2}$ in $0.3 \mathrm{M} \mathrm{NaCl}$ solution $\left(1 \mu \mathrm{Ci} 63 \mathrm{Ni} / 500 \mu \mathrm{L}, 1 \mathrm{nCi}{ }^{59} \mathrm{Ni} / 500 \mu \mathrm{L} ; \sim 200 \mu \mathrm{g}\right.$ total $\mathrm{Ni}$ ) or ${ }^{\text {nat }} \mathrm{NiCl}_{2}$ in $0.3 \mathrm{MNaCl}$ solution $(200 \mu \mathrm{g} \mathrm{Ni})$. Each animal is housed in metabolism cages and given free access to regular feed and water. After 24 hours, the animals are sacrificed and the collected samples (spleen, kidney, heart, lungs, brain, blood, urine, feces) are stored in scintillation vials in a commercial freezer.

Tissue Processing- in development. We have experimented with different methods of decomposing the organic matter. Wet ashing of the 1 gram tissue samples with $5 \mathrm{ml}$ of concentrated $\mathrm{HNO}_{3}, 10 \% \mathrm{H}_{2} \mathrm{O}_{2}$ or combinations of the two reagents resulted in $70 \%$ recovery of the $1 \mathrm{mg} \mathrm{Ni}$ that was added to the tissue. A dry ashing technique in which the tissue sample was heated in a tube furnace with a low $\mathrm{O}_{2}$ flow resulted in a $35 \%$ loss of $\mathrm{Ni}$. At present, we are developing microwave digestion techniques. Microwave digestion appears to be optimum process because the closed system provides high temperatures and pressures insuring complete digestion of organic material without the loss of sample. 


\section{Results.}

${ }^{59} \mathrm{Ni}$ AMS results using orepared standards and blanks.

Samples were prepared from the standard dilution series. Figure 3 contains data from the blanks $\left({ }^{59} \mathrm{Ni} / \mathrm{Ni}=0\right)$ measured on a single day. Measured ${ }^{59} \mathrm{Ni} / \mathrm{Ni}$ ratios for the $1 \times 10^{-10}$, $1 \times 10^{-9}$ and $1 \times 10^{-8}$ standards are shown in Figure 4.

\section{Iracer results.}

One experiment with four animals has been conducted. ${ }^{63} \mathrm{Ni}$ liquid scintillation counting of partially digested tissues indicates that $10 \%$ of the $\mathrm{NiCl}_{2}$ dosing solution was in the urine of the animals. Further LSC and AMS analyses of these samples will be made after tissue digestion protocols have been fully developed.

\section{Discussion.}

Discussion of Ni AMS results.

Measuring a non-zero ${ }^{59} \mathrm{Ni} / \mathrm{Ni}$ for the blanks suggests that when we calibrate the AMS instrumentation, we are not filtering out all of the stable nickel $\left({ }^{58} \mathrm{Ni}\right)$. We are still working on ways to prevent the stable nickel from getting through and being measured at the $\mathrm{x}$-ray detector.

Data from the $10^{-10}, 10^{-9}$ and $10^{-8}$ measurements indicate that we are getting better than $10 \%$ reproducibility. With the elimination of stable nickel being measured by the $x$-ray detector, experience in tuning the instrumentation to measure for ${ }^{59} \mathrm{Ni}$ and changing a component in the x-ray detector, we expect an improvement in the precision and accuracy of our measurements.

\section{Discussion of tracer results.}

Data from the tracer experiments can be compared to data reported by Borg-Neczak, et al 4 in which $200 \mathrm{~g}$ rats were dosed with $20 \mu \mathrm{Ci}$ of ${ }^{63} \mathrm{NiCl}_{2}$ solution. Borg-Neczak, et al reported that after 24 hours, a significant amount of ${ }^{63} \mathrm{Ni}$ was found in the kidney. With LSC of partially 
digested tissue, we found no ${ }^{63} \mathrm{Ni}$ in the kidneys. There are two possible explanations for our observation. The first possibility is that because the dosing solution was approximately $6 \mathrm{M}$ $\mathrm{NaCl}$, the majority of the tracer was not absorbed but instead excreted in the urine of the rat. $63 \mathrm{Ni}$ was found in the urine but the total $1 \mu \mathrm{Ci}$ dose was not detected. We have modified the dosing solution to be $0.3 \mathrm{M} \mathrm{NaCl}$. The second possibility is that incomplete digestion of tissues and body fluids results in an interference and less than $100 \%$ efficiency in the counting of decays by LSC. We have experimented with wet acid and dry ashing techniques to decompose the organic material and facilitate the extraction of nickel from tissues. We found that nickel was lost during these procedures. As mentioned, we are presently developing microwave digestion techniques.

\section{Conclusions.}

Our experiments suggest that wa can measure ${ }^{59} \mathrm{Ni} / \mathrm{Ni}$ ratios as low as $1 \times 10^{-10}$ with a detection limit of $\pm 3 \times 10^{8}$ atoms. We use projectile $x$-rays to detect nickel and cobalt atoms and by subtracting the cobalt tail from the nickel peak, we quantify the number of the nickel atoms getting through the system. Purification of the nickel solution with nickel carbonyl generation is needed to insure that cobalt levels do not severely exceed the amount of nickel in the sample.

Our progress towards the biomedical tracer application of $59 \mathrm{Ni}$ AMS continues. It is obvious that we need digestion methods that will provide complete recovery of nickel from the tissues and body fluids. Experiments using ${ }^{59} \mathrm{Ni}$ AMS to measure the uptake of nickel in layers of the skin are a possibility.

\section{References.}

1 J.E. McAninch, G.S. Bench, S.P.H.T. Freeman, M.L. Roberts, J.R. Southon, J.S. Vogel, I.D. Proctor. Nuclear Instruments and Methods 99, 541 (1995).

2 J.S. Vogel, K.W. Turtletaub, R. Finkel, D.E. Nelson. Analytical Chemistry 67,353 (1995).

3 R.E.Sturgeon, S.N. Willie, S.S. Berman. J. Analytical Atomic Spec., 4, 443 (1989).

4 K.Borg-Neczak, H. Tjalve. Archives of Toxicology, 68, 450 (1994). 


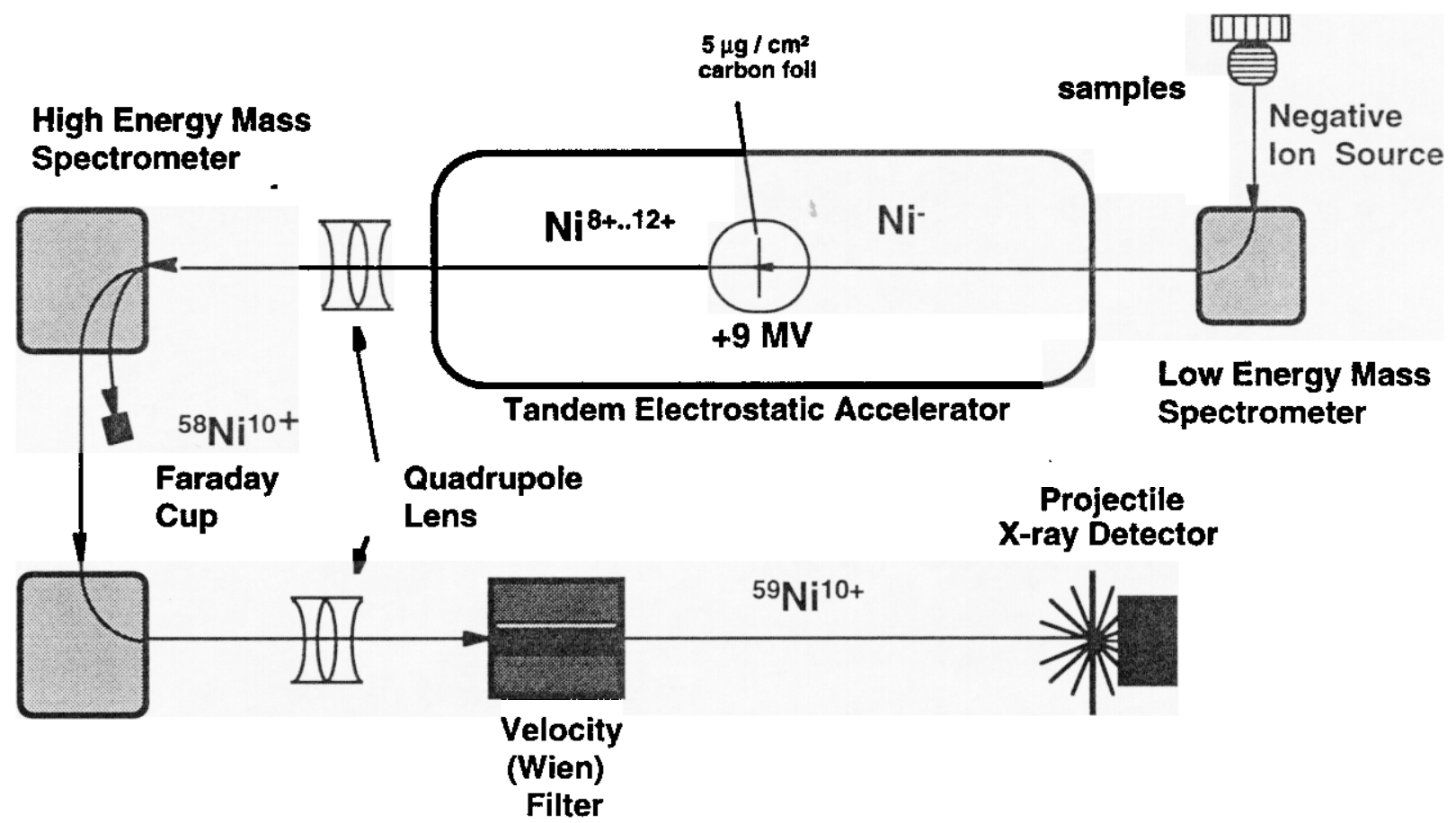

Figure 1.

AMS and Projectile X-ray Detector.

Negative nickel ions get sputtered out the sample and filtered through various low energy filters before proceeding through the accelerator. The accelerator is a tandem electrostatic accelerator; It strips the nickel particle of electrons. After passing through the accelerator, the nickel is positively charged and at a high energy. The stable nickel $\left({ }^{58} \mathrm{Ni}\right)$ is measured in a Faraday cup and the rest of the nickel beam gets filtered with various electromagnetic devices. The devices are calibrated to allow only ${ }^{59} \mathrm{Ni}$ in the $10+$ charge state to make it through to the $\mathrm{x}$ ray detector. The $\mathrm{X}$-ray detector measures the number of atoms making it through the system. $\mathrm{X}$-rays energies are characteristic for an element. Both nickel and cobalt $\mathrm{X}$-rays are measured. Because these spectra overlap, subtraction of the cobalt tail from the nickel peak gives the corrected measure of the number of nickel atoms detected. 


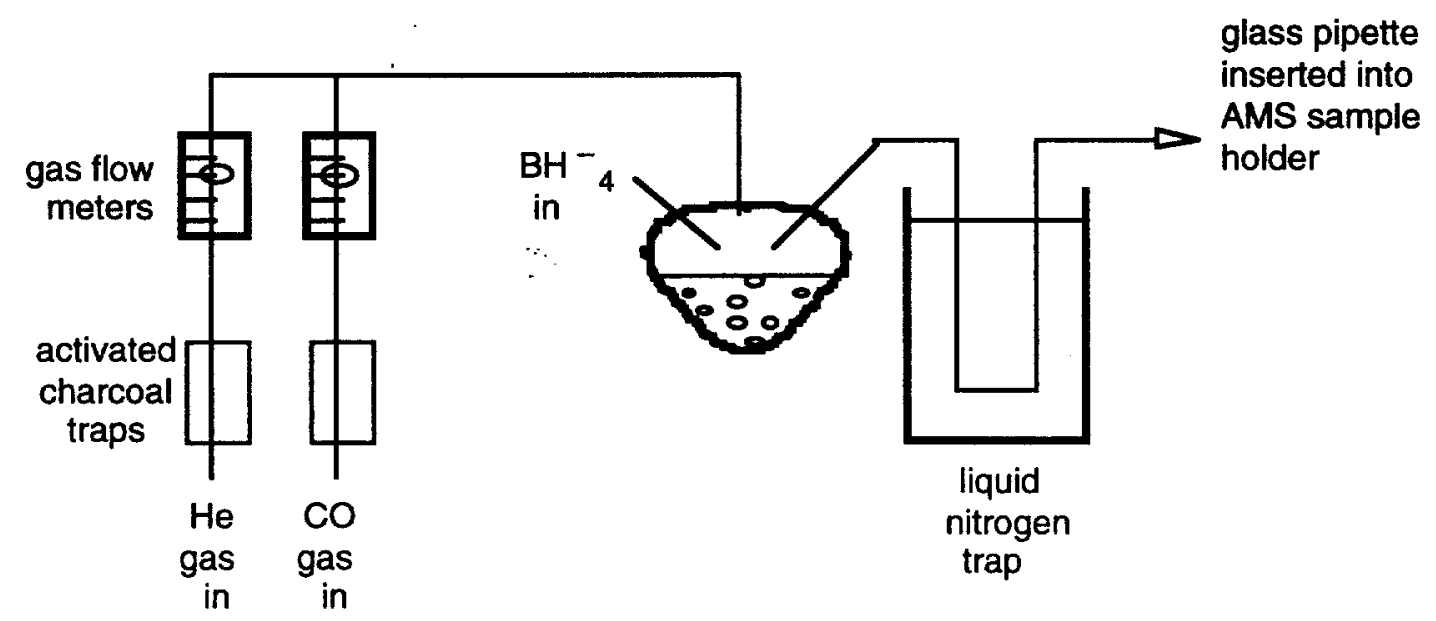

$\mathrm{Nill}^{\prime} \rightarrow \mathrm{Ni}^{0} \rightarrow \mathrm{Ni}(\mathrm{CO})_{4} \rightarrow$ metallic $\mathrm{Ni}$

Figure 2.

Nickel Purification Process: $\mathrm{Ni}(\mathrm{CO})_{4}$ Generation.

Adapted from Sturgeon, et al., J. Anal. Atomic Spec. 4, 443 (1989), this process is used to remove cobalt from the nickel solution before it gets put into the sample holder as metallic nickel. This process works because nickel forms a tetracarbonyl preferentially over the other metal of concern, cobalt.

The reactor vessel is a standard heart shaped glass beaker with three female openings. The " $U$ " shaped tube has an inner diameter of $1 \mathrm{~cm}$ and contains silanized glass beads. The arrow leading to the AMS sample holder in the diagram is a glass pipette with its outer diameter altered so it can be inserted into the sample holder for decomposition of $\mathrm{Ni}(\mathrm{CO})_{4}$. 


\section{Figure 3.}

\section{AMS Measurement of Blanks $\left({ }^{50} \mathrm{Ni} / \mathrm{Ni}=0\right)$}

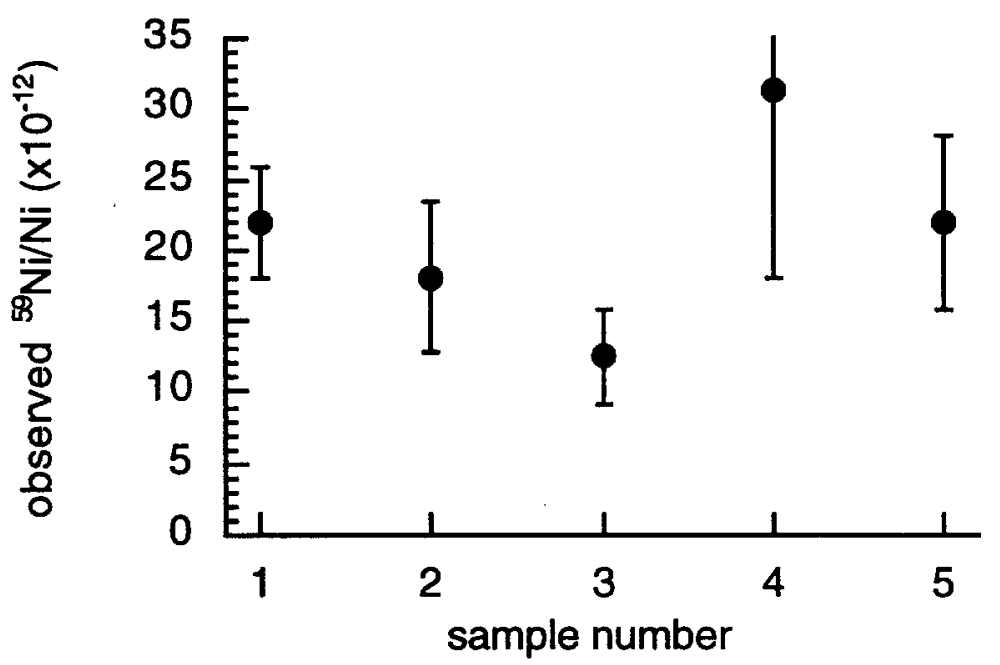

Figure 3 shows the ${ }^{59} \mathrm{Ni} / \mathrm{Ni}$ values measured from the blanks $\left({ }^{59} \mathrm{Ni} / \mathrm{Ni}=0\right)$. The nonzero values suggest we could be measuring stable $\mathrm{Ni}$ at the $\mathrm{x}$-ray detector.

Figure 4.

AMS Measurements of $10^{-10}, 10^{-9}, 10^{-8}{ }^{59} \mathrm{Ni} / \mathrm{Ni}$.

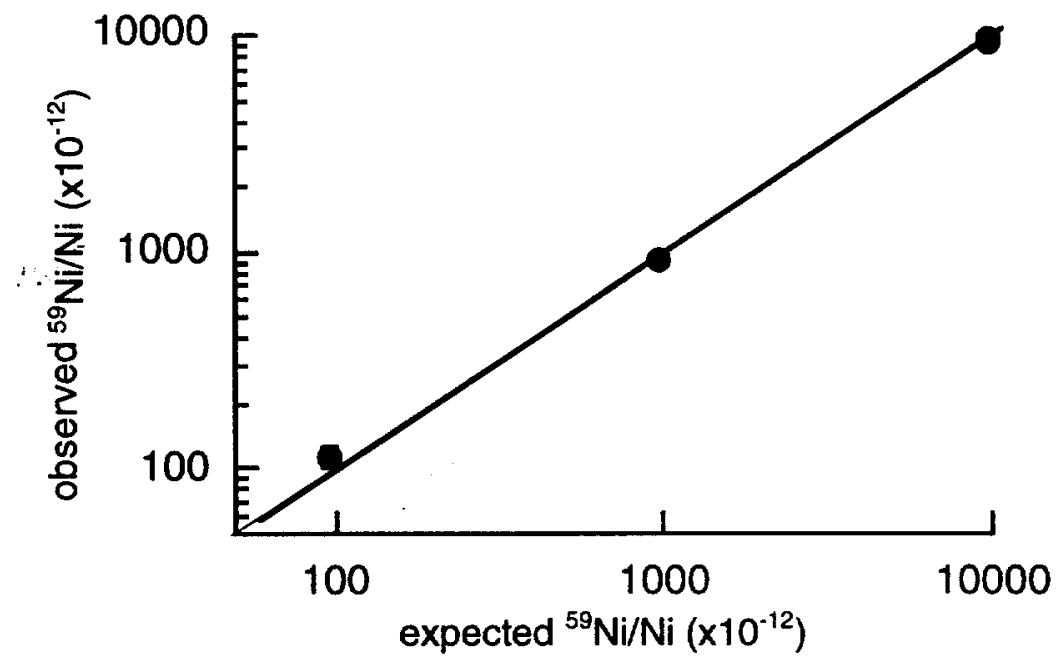

Figure 4 contains the ${ }^{59} \mathrm{Ni} / \mathrm{Ni}$ measurements from the prepared standards. The observed values come within $10 \%$ of the expected values. 


\title{
Inverse Kinematic Program for a Robotic Manipulator
}

\author{
Xerxes T. Helms \\ California State University, Sacramento \\ School of Engineering and Computer Science \\ Department of Electrical and Electronic Engineering \\ Lawrence Livermore National Laboratory \\ Livermore, California 94550
}

May 8, 1996

Prepared in partial fulfillment of the requirements of the Science and Engineering Research Semester under the direction of Shin-Yee Lu Research Mentor, in the Lawrence Livermore National Laboratory.

* This research was supported in part by an appointment to the U.S. Department of Energy Science and Engineering Research Semester (hereinafter called SERS) program administered by LLNL under Contract W-7405-Eng-48 with Lawrence Livermore National Laboratory. 


\section{Abstract}

- The objective of this project is to develop a program that can solve the inverse kinematic equations for a high precision redundant robotic manipulator system.

- The program uses plane geometry along with vector mathematics to solve the manipulator's equations.

- The program will calculate the joint angles for a wide range of manipulator positions and produce a graphical representation of the manipulator's position.

- Using the program with its specifically developed manipulator any operator can analyze the manipulator's position and develop specialized maneuvers. 
Introduction

The program developed for this project was designed to solve the inverse kinematic equations for a redundant high-precision robotic manipulator. This program is necessary to provide the operator with a way to create precise movement for the manipulator. The program uses both plane geometry and vector mathematics to create the inverse kinematic equations for the manipulator. Finally, it was tested using trial and error along with the process of feeding the output into a forward kinematic program and comparing the end effector's position.

Robotic analysis has many terms specific to its field such as end effector, frame of reference, kinematic decoupling, translation matrix, rotation matrix, and direction vector. An end effector is any device that interacts with the physical environment. Generally an end effector will be a set of fingers or gripper, however it can also be a welding torch or another type of tool. A frame of reference is a coordinate system that has been assigned to a joint in the manipulator. Each joint has a specific frame of reference that will be used to develop the describing kinematic equations. Kinematic decoupling describes the situation where a portion of the manipulator is unrelated to another portion kinematically. If a system can be decoupled it will be easier for the engineer to create the describing kinematic equations because the equations will involve fewer variables. A translation matrix is a four by four matrix that results from moving from one frame of reference to another, see figure 1. 


$$
\begin{aligned}
& -C 5-S 50 \\
& \text { Rotation matrix: } \quad-S 5 \quad C 5 \quad 0 \quad \text { Direction vector: } \quad \begin{array}{lllll}
0 & L 4 & 04
\end{array} \\
& \begin{array}{lll}
0 & 0 & 1
\end{array} \\
& \mathrm{~T}_{45}=\begin{array}{cccc}
-C 5 & -S 5 & 0 & 0 \\
-S 5 & C 5 & 0 & L 4 \\
0 & 0 & 1 & 04 \\
0 & 0 & 0 & 1
\end{array}
\end{aligned}
$$

Figure 1: Matrix definitions.

The rotation matrix represents the rotation of the fifth frame of reference about the fourth frame. The direction vector represents a vector pointing from the origin of frame four to the origin of frame five. The final matrix, $T_{45}$, is a translation matrix.

Robotic manipulators are created from links that are connected by joints to form open kinematic chains. To completely describe the chain of motion an engineer must create two sets of equations. First the chain is described using forward kinematic equations. These equations use angles to describe the position and orientation of the end effector. The second method of describing the motion is through the inverse kinematic equations. This set of equations finds the joint variables in terms of the end effector position and orientation. It is generally much more difficult to create the inverse kinematic equations than the forward kinematic equations.

The reason for developing this program was two fold. The program will get the most use by providing a way to create precision motion. When integrated with the motion control software that controls the manipulator the program will allow the operator to generate a smooth path for the arm to follow. Without the assistance of this program the current software is unable to make the simplest movement, that of a straight line. It is imperative that the manipulator be capable of moving in a straight line for insertion and extraction purposes. The program has the 
secondary function of allowing further analysis of the physical manipulator. It will allow the characteristics of the manipulator's motors to be described with equations. It is able to do this because it is an ideal model for the manipulator and any deviation between the manipulator and the program results from the motor's characteristics. The program will also allow the verification of the accuracy of the manipulator.

The robotic manipulator is a high-precision redundant arm, see figure 2 . This manipulator is capable of 10 micron accuracy over at least an $\mathbf{8}$ hour period. This is achieved through direct drive motors with high precision gear heads. The manufacturer, Harmonic Drive, produced the gear heads that have a gear ratio of 100:1. This high reduction ratio combined with the basic design of the gear head reduces backlash to the minimum. The manipulator is considered redundant because it has seven degrees of freedom, instead of the six that are typical of a robotic manipulator. Six degrees of freedom is the minimum number required to reach any point in space with any orientation. The seventh degree of freedom in this manipulator allows the user to set one variable before the program solves for the joint variables, see figure 3 .

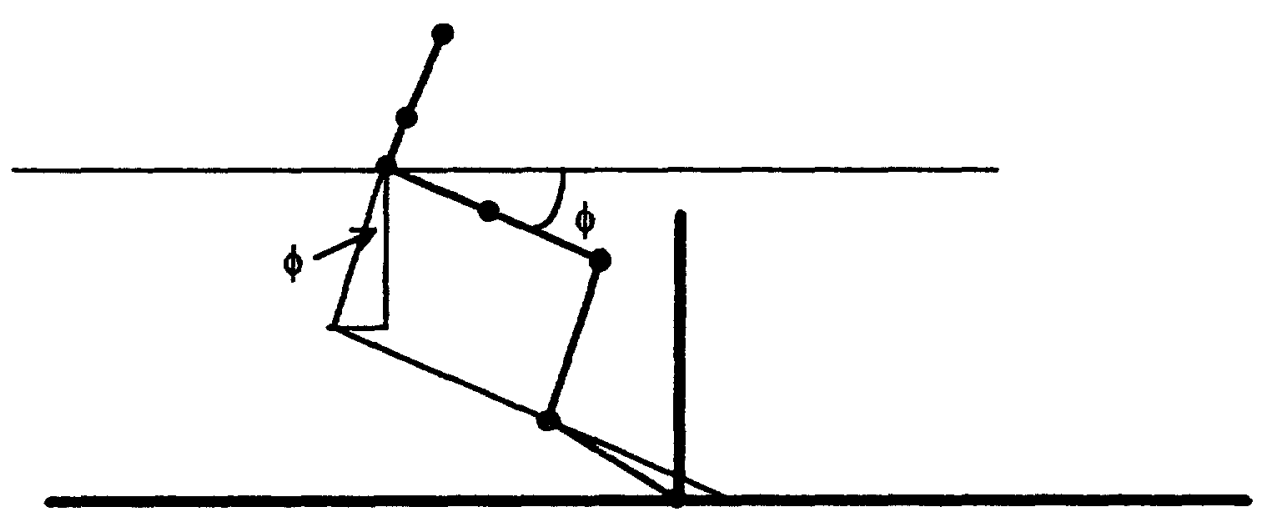

Figure 3: Free variable set by user represents angle of intersection of vertical plane and and horizontal plane. 


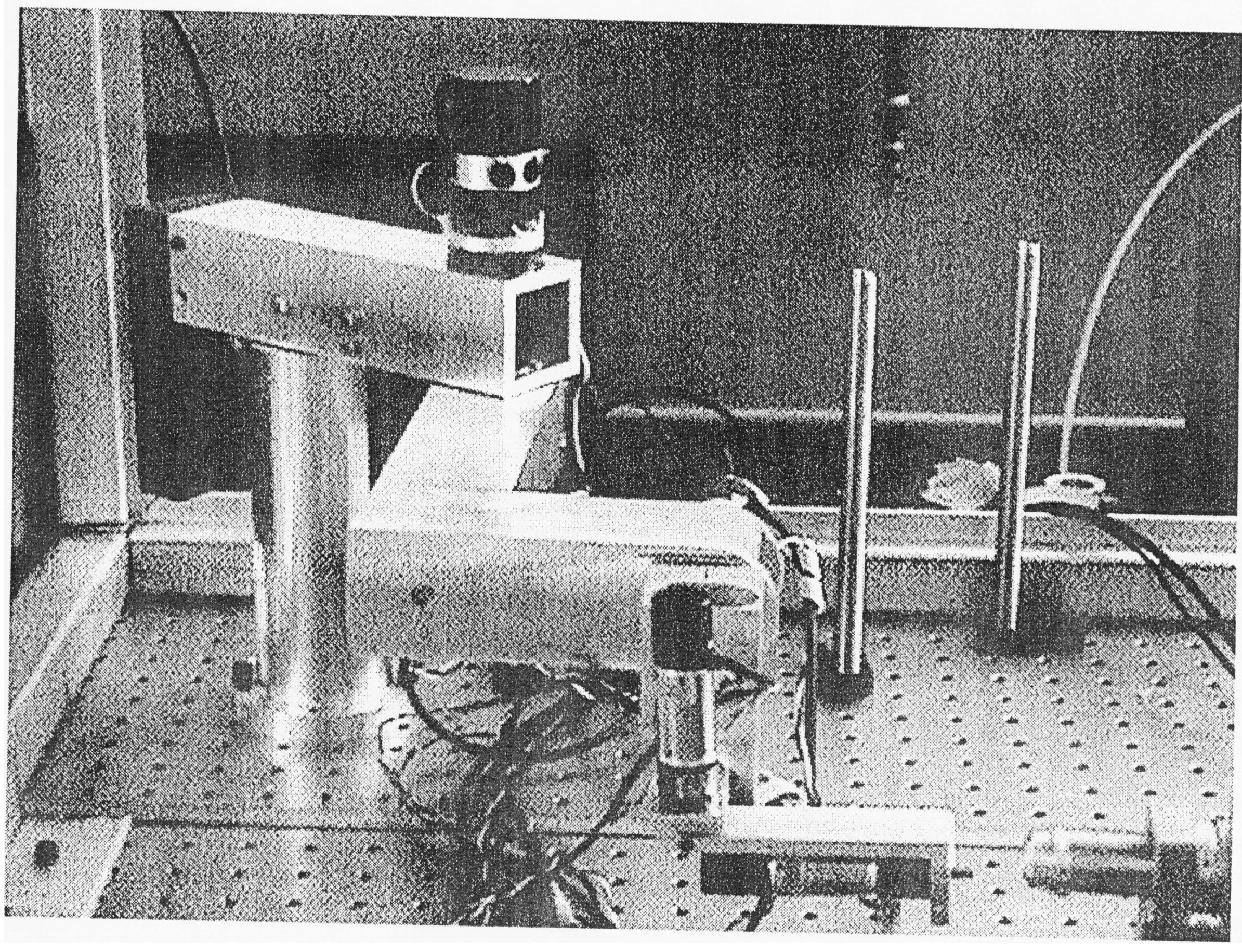

Figure 2: Robotic Manipulator. 


\section{Methods}

There are two methods to create inverse kinematic equations. The first compares elements of forward kinematic matrices to solve the joint variables. This method compares the orientation matrix of the end effector with the matrix resulting from the multiplication of the translation matrices. If this method is used to solve all the joint variables at once, the engineer will end up with $\mathrm{n}$ simultaneous equations to solve, where $\mathrm{n}$ is the number of joints in the manipulator, usually six. This method is typically unsatisfactory because the equations are too difficult to solve, however it will work for situations where $\mathrm{n}$ is small, such as creating the equations for the manipulator's wrist.

The second method, and the method used for this program, is graphical and uses the manipulator's design to create the inverse kinematic equations. The graphical method uses plane geometry to solve the joint variables in terms of the position and orientation of the end effector. The inverse kinematic equations take advantage of the fact that the phase variable is set before any calculations are made. The program also takes advantage of the fact that link one and link two describe a horizontal plane while link three and link four describe an intersecting vertical plane, see figure 4. Links' three and four are decoupled from links' one and two as a result of these two planes of action. This means that the law of cosine can describe theta one and two, and it can describe theta three and four without involving theta one and two in the equations.

The redundant aspect of this manipulator was the most difficult to deal with from the programming aspect. Fortunately, most of the groundwork was done by a previous CO-OP student from another University. However, her work was for a previous incarnation of the manipulator, one that had four links, and she had written the program for a limited range of 
motion. Her approach to the problem was graphical in nature and relied heavily on plane geometry to determine the solution for the manipulator. The user would choose the angle of intersection of the planes, the phase angle, and the end point coordinates. The program then solved the equation of the intersection line in the $x-y$ plane using the line equation, $y=m x+b$. Next it used several other equations to solve the first joint variable. Once the first angle was found the program solved the second angle by finding the coordinates of the joint positions and using the law of cosine formula. The third and fourth joint variables were found in a similar manner.

The changes necessary to update the program for the newest version of the arm included adding three more degrees of freedom, updating the link sizes and offset changes, and expanding the workspace. These three modifications were made in two stages. The first stage included

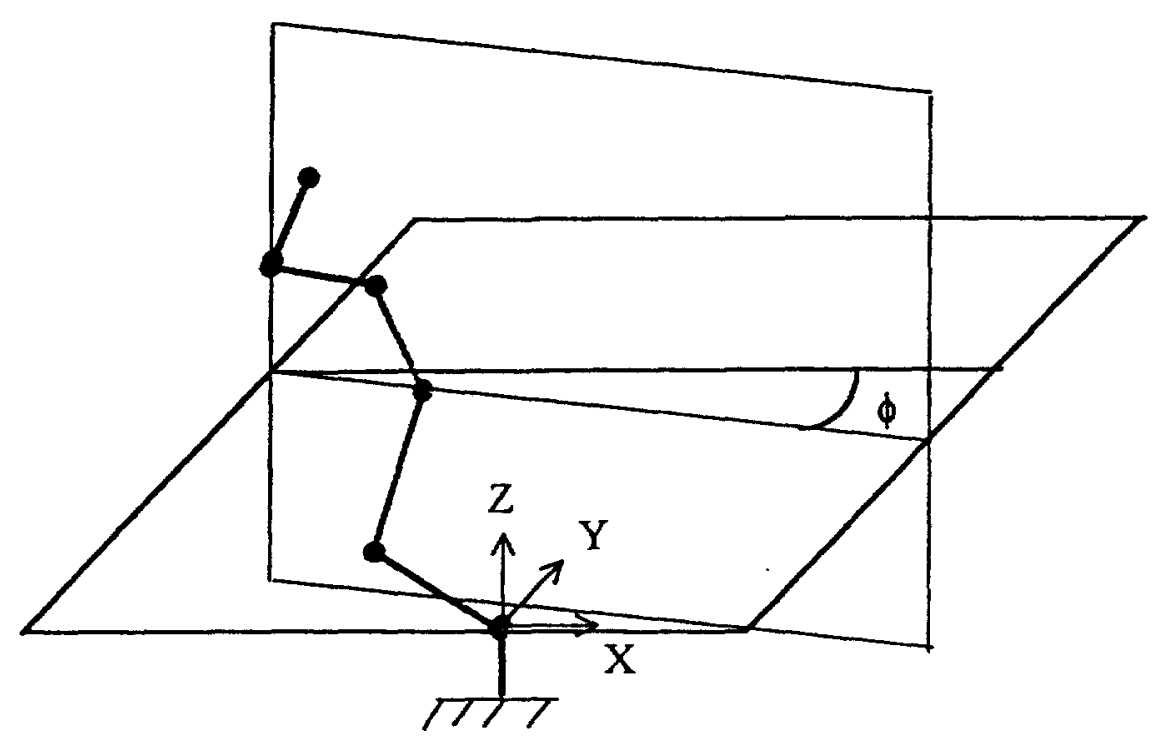

Figure 4: Links 3 and 4 represent a vertical plane intersecting the horizontal plane created by links 1 and 2 .

updating of the link lengths and offsets along with the expansion of the workspace. It was found 
that the original program failed for many different configurations. Most of these configurations were singularity points, that is a position in space where only one solution for the manipulator is possible. The rest of the configurations failed because they were outside the program's workspace. The program was modified to take care of the singularities by separating the calculations in to three groups:

1) a phase angle of zero degrees;

2) a phase angle of 90 degrees;

3) any other phase angle up to 180 degrees.

The first two groups were able to capitalize on the manipulator's geometry to make many of the calculations simple. The third group relied on the original program to make the necessary calculations. The workspace was expanded to include the entire frontal volume of the manipulator. The program also implemented a checking mechanism to verify the input position was within the arm's workspace. In addition to the above modifications the program was rendered capable of displaying a line plot of the manipulator's position in the $x-y$ plane and a view of the link 3-4 plane to aid with general analysis, see figure 5 .

The second stage of modification dealt with the three additional degrees of freedom for the wrist portion of the manipulator. The modification started with the addition of the final frame's orientation, relative to the base of the robot, as additional user input. Given that information, the program was able to use vector math to calculate the joint locations to the fifth reference frame where the updated version of the original program takes over. The difficulties for this stage were caused by the use of the dot product to determine the joint angle. This method only gives positive answers. To determine the sign of the angle the cross product was 

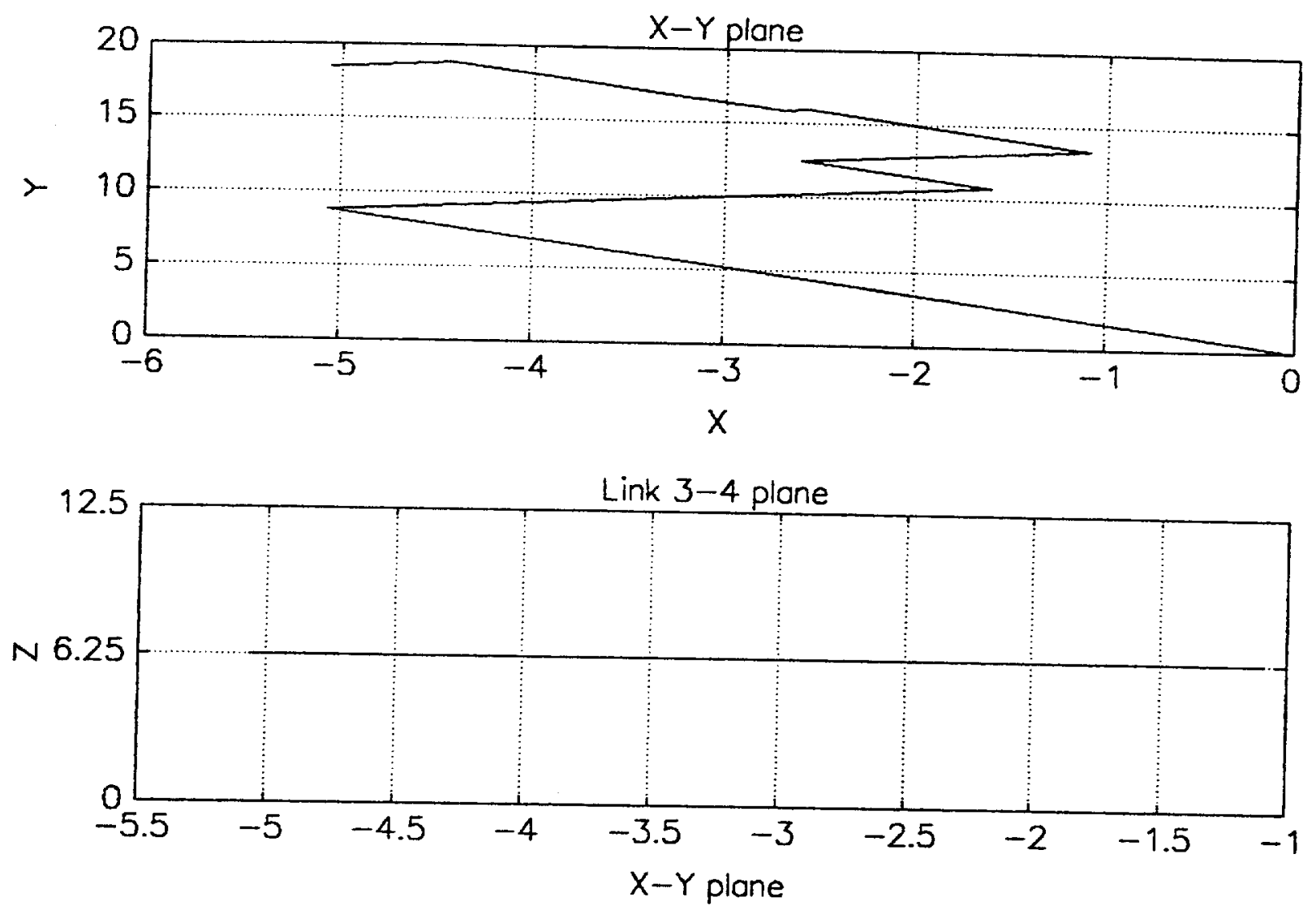

Figure 5: Matlab printout of program output. 
used to find the third reference vector for the frame. The dot product was used again to get a reference angle to determine which direction the next frame had rotated. The sign of the angle was based on information from the second dot product.

To test the program two methods were used. The first was an ongoing process of trial and error. A configuration was entered into the program and the results were analyzed to see if the output matched the expected output. Since the configuration was determined prior to using the program any deviation in output was an indication of an error in the program. These errors were then isolated and corrected. The result of this type of testing was a robust program with few errors. One problem with this method however, is that only a limited number of configurations can be tested. Thus there is the possibility that some unique configurations will create situations where the program will fail.

To attempt to correct the problem's associated with the trial and error portion of the testing a second method was used. The program was run normally but when finished it would send the calculated angles to a forward kinematic function. The function would use the given angles to calculate the endpoint. This endpoint was sent back to the main program and compared to the original endpoint entered by the user. Using this method any configuration could be entered without prior calculation. The output determined if there were any errors in the program. Unfortunately, to ensure the forward kinematic function was working properly the trial and error method was used. This process was still under testing when this paper was written. No random configurations were entered for testing.

In conclusion the development of this program was the next step needed to allow this manipulator to go onto the market. The equations written for this program use graphical 
methods to solve the joint variables of the manipulator in terms of the end effector position and orientation. The equations were tested using trial and error along with comparing the program's input to the output of a forward kinematic program. This program will be used to create precision movement, such as moving in a straight line. It will also be used to further analyze the physical manipulator. 


\section{Acknowledgments}

The author would like to thank the Department of Energy's Science and Engineering Research Semester Coordinator at Lawrence Livermore National Laboratory, Barry Goldman, for making this research semester possible. Special thanks to K. David Young for assistance with the technicalities of robotic analysis, Shin-yee $\mathrm{Lu}$, for allowing me the opportunity to work under her supervision, and to all the people who are part of the Intelligent Mechatronics Laboratory. 
Bibliography

1) Spong,M.W., Robot Dynamics and Control, John Wiley and Sons, NY, 1989.

2) Lewis, F.L. et al., Control of Robot Manipulators, MaCmillan Publishing Company, NY, 1993.

3) Paul, R., Robot Manipulators: Mathematics, programming, and control, The MIT Press, MA, 1981. 


\title{
Lantz E. Johnson \\ Kent State University
}

Lawrence Livermore National Laboratory

Science and Engineering Research Semester

Chemistry and Material Science Department

\author{
Mentor: Dr. Alison Chaiken
}

Temperature Dependence of NiO/NiFe Bilayer for Giant Magnetoresistance Spin-Valves

08 May 1996 
TABLE OF CONTENTS

TITLE PAGE

ABSTRACT

INTRODUCTION

WHAT A GMR SPIN-VALVE IS

THE IBS SYSTEM

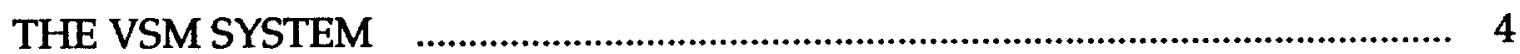

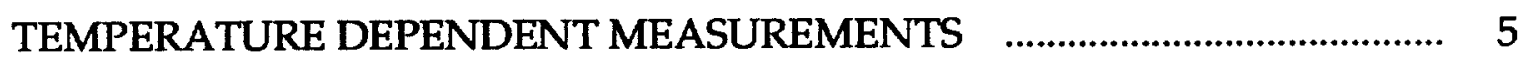

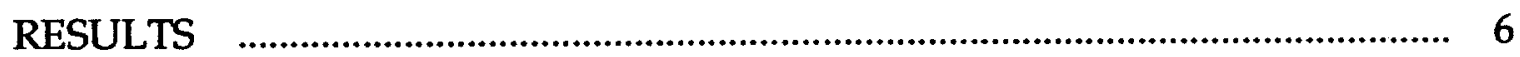

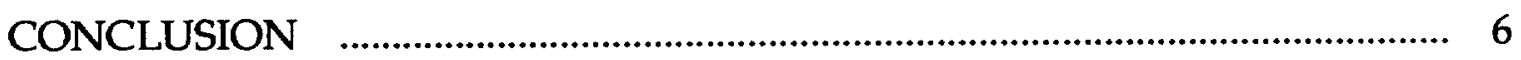

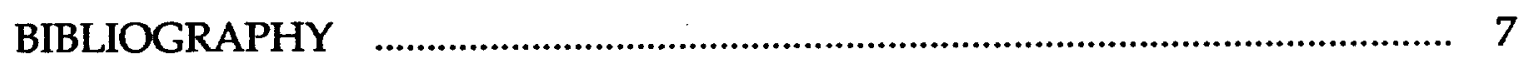

\section{LIST OF FIGURES}

GMR SPIN-VALVE

IBS SYSTEM

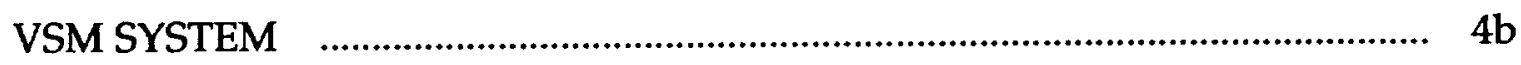

VSM / W OVEN

EXCHANGE COUPLING

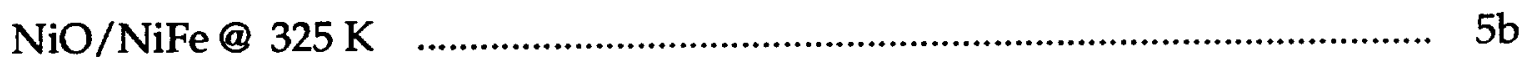

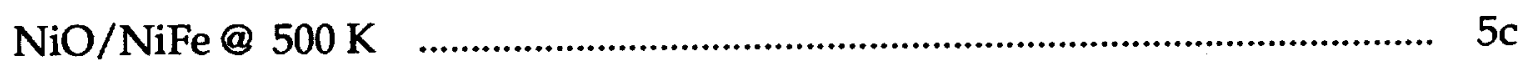

Hc \& He vs. T 


\title{
TEMPERATURE DEPENDENCE OF NiO/NiFe BILAYER FOR GIANT MAGNETORESISTANCE SPIN-VALVES
}

\author{
Lantz E. Johnson \\ Kent State University \\ Lawrence Livermore National Laboratory \\ Livermore, California 94550
}

08 May 1996

Prepared in partial fulfillment of the requirements of the Science and Engineering Research Semester under the direction of Dr. Alison Chaiken, Research Mentor, in the Lawrence Livermore National Laboratory.

* This research was supported in part by an appointment to the U.S. Department of Energy Science and Engineering Research Semester (hereinafter called SERS) program administered by LLNL under Contract W-7405-Eng-48 with Lawrence Livermore National Laboratory.

If this paper is to be published, a copyright disclaimer must also appear on the cover sheet as follows:

By acceptance of this article, the publisher of recipient acknowledges the U.S. Government's right to retain a nonexclusive, royalty-free license in and to any copyright covering this article. 


\title{
Lantz E. Johnson
}

\section{Temperature Dependence of NiO/NiFe Bilaver for Giant Magnetoresistance Spin-Valves}

\begin{abstract}
:
The temperature dependence of $\mathrm{NiO} / \mathrm{NiFe}$ thin film bilayers is investigated. The films are grown by direct ion beam sputtering (IBS) of a $\mathrm{NiO}$ and NiFe target and magnetic measurements are made with a vibrating sample magnetometer (VSM) equipped with a high temperature oven. The results of these measurements show the exchange field (He) decreasing approximately linearly with increasing temperature dropping close to zero near the Neél transition temperature for $\mathrm{NiO}$ which is $520 \mathrm{~K}$. This linear relationship occurs over the temperature range of a typical operating read head device and therefore needs to be considered when using this material for a GMR spin-valve read head devise. The coercivity (Hc) also decreases with increasing temperature but at a slower rate reaching the typical room temperature value of a free NiFe layer ( 1 - $2 \mathrm{Oe}$ ). The coercivity is less than the exchange field value over the temperature range of a typical operating read head device indicating this material to be stable for use as a GMR spin-valve read head device.
\end{abstract}




\section{INTRODUCTION:}

The interest in studying giant magnetoresistance (GMR) spin-valves comes from the ever increasing desire to store more information on disks. Information is represented in the form of magnetic bits and therefore to store more information more bits are needed on the disk. One way to do this is to make the bits smaller, however there is a problem associated in doing this. By decreasing the bit size the amount of magnetic material per bit is also decreased resulting in a corresponding reduction in detected magnetic signal. A more sensitive read head is therefore required to detect this ever weakening signal. Giant magnetoresistive materials promise to increase sensitivities in read head devices by orders of magnitude over today's standard thin-film inductive read heads.

This paper discusses initially some background on what a GMR spinvalve basically is, the ion beam sputtering system used to grow magnetic thin films, and the vibrating sample magnetometer used to perform magnetic measurements on the samples. The temperature dependent measurements made on $\mathrm{NiO} / \mathrm{NiFe}$ bilayers are then discussed along with the results of these measurements and what was learned from them.

WHAT A GMR SPIN-VALVE IS:

A giant magnetoresistance spin-valve is a multilayer thin film device. In a simplistic model it consists basically of four layers (fig. 1). The bottom antiferromagnetic $\mathrm{NiO}$ layer interacts with the ferromagnetic NiFe layer on top of it through exchange coupling. This causes the magnetic moment of the NiFe to be pinned in one direction. The top ferromagnetic NiFe layer's magnetic moment is allowed to move freely and independently of the bottom NiFe layer. When the device senses a bit the top layer's moment moves so that the two ferromagnetic layer's moments are either parallel or antiparallel to one another. Due to the different spin condition of the conduction electrons that is associated with the parallel and antiparallel orientations of the two ferromagnetic layer's magnetic moments there is a different value of electrical resistance associated with these two orientation. This difference in electrical resistance is a giant change and hence the name giant magnetoresistance spin-valve. 
m
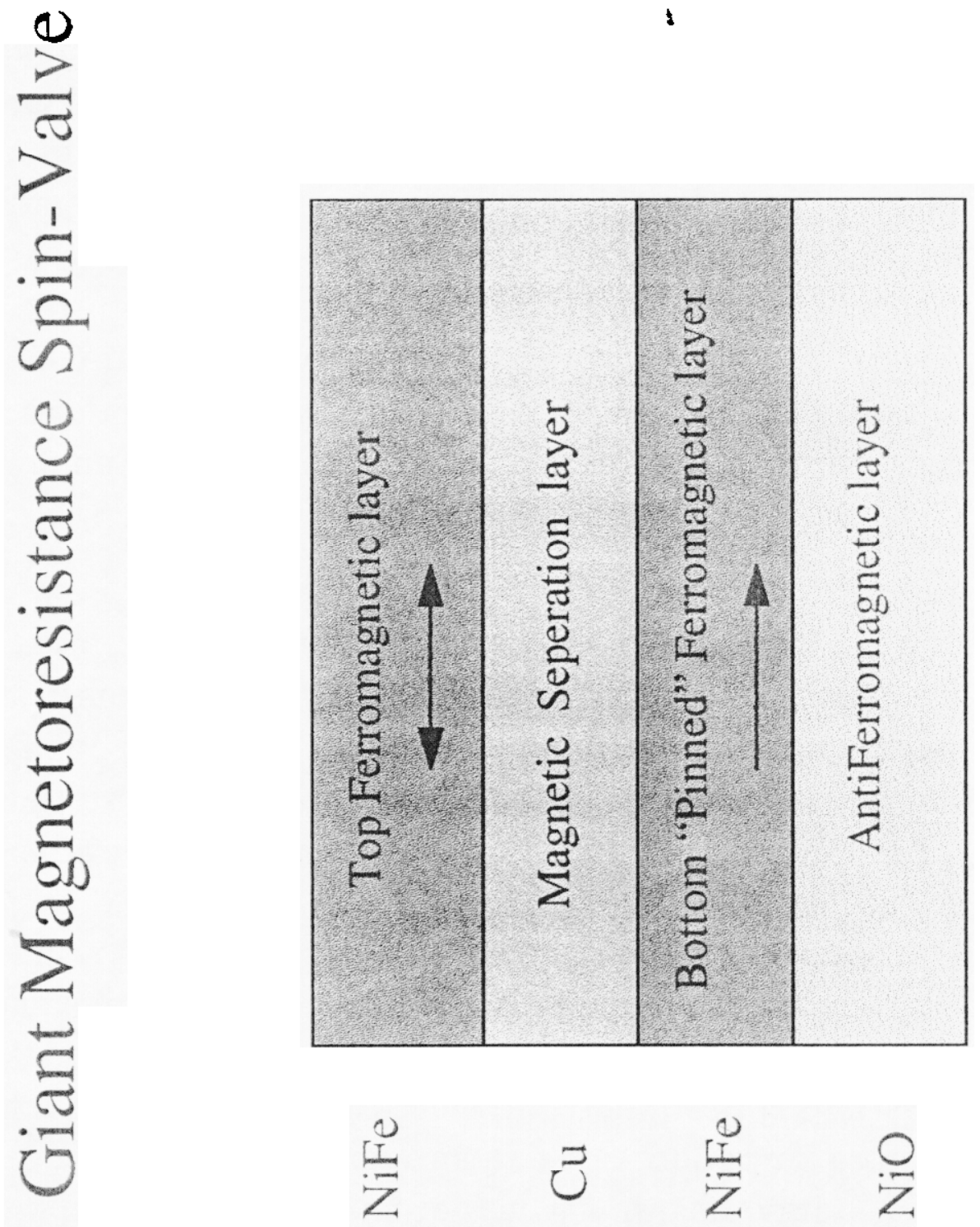

$\frac{O}{E}$ 


\section{THE IBS SYSTEM:}

The ion beam sputtering system (fig. 2) used to grow our magnetic thin films employs a tungsten filament inside the ion gun to produce electrons which then ionize Ar gas supplied to the gun at a partial pressure of $2.8 \mathrm{x}$ $10^{-4}$. These Ar ions are accelerated striking the targets which causes material to be ejected from the targets and accumulate on the substrates $25 \mathrm{~cm}$ above through a small hole in the cooling tray. The cooling tray is cooled by liquid nitrogen to improve the vacuum of the deposition chamber. The substrate's temperature ,approximately $60^{\circ} \mathrm{c}$, is controlled by a heater and the thickness of the deposition is monitored by a quartz crystal oscillator with deposition rate being $0.1 \AA / \mathrm{sec}$. The whole system can be controlled from a computer to automate it.

\section{THE VSM SYSTEM:}

The magnetic properties of our samples are measured via the vibrating sample magnetometer (fig. 3,4 ) which is equipped with a high temperature oven for making temperature dependent measurements. An electromagnet at the base of the instrument that can reach field strengths up to 6000 Oe is used to apply an external field to the sample. The change in magnetization of the sample is then measured as the applied field is varied. This change in magnetization is measured through the principle of induction and is accomplished in the vsm by attaching our sample to the end of a long rod which is then vibrated up and down inside of $\mathrm{x}$ and $\mathrm{y}$ direction coils located between the poles of the electromagnet. The resulting signal is translated to read out on a computer as the hysteresis of the magnetic thin film. 


\section{Ion Beam Sputtering System}

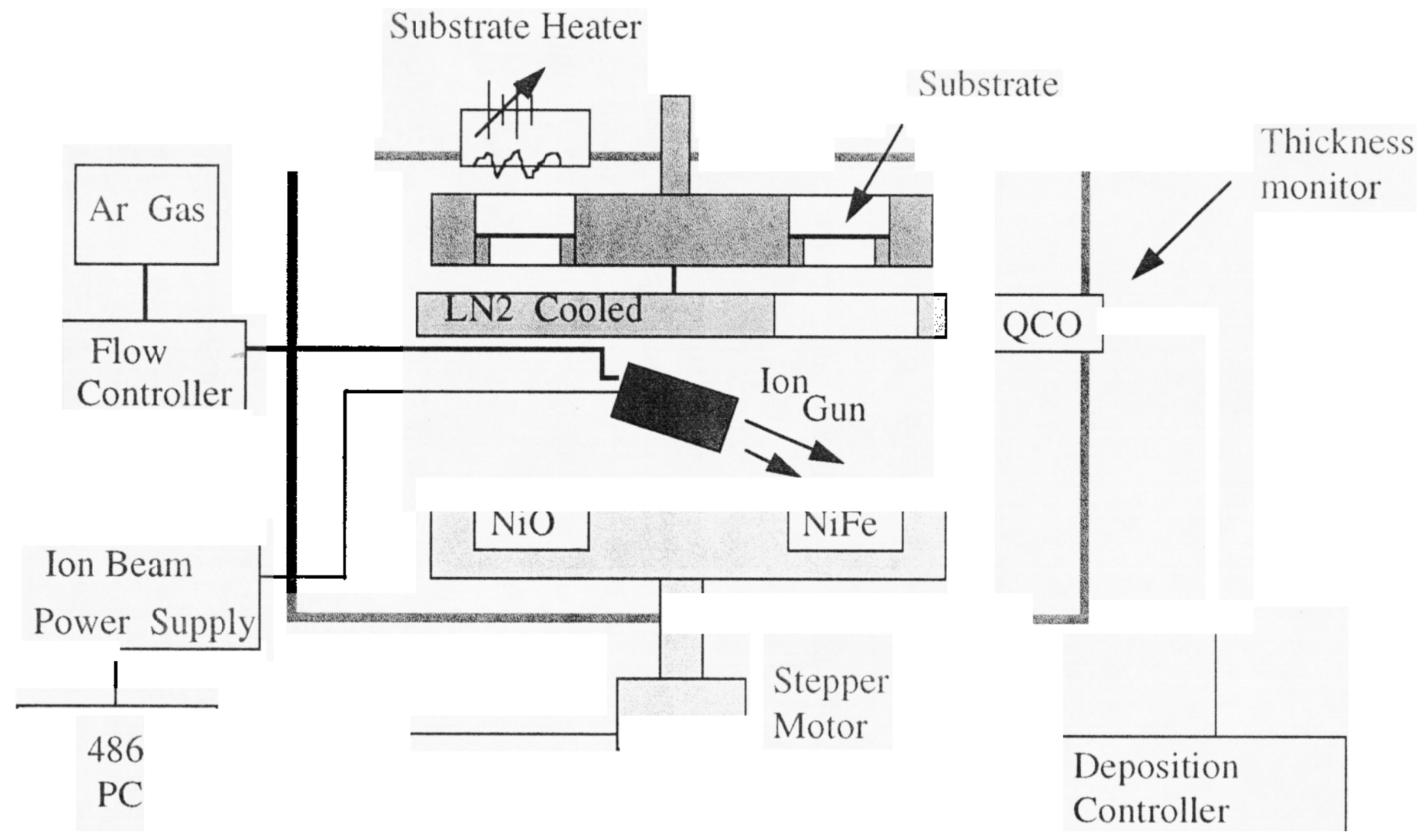

(FIG. 2) 
Vibrating Sample Magnetometer

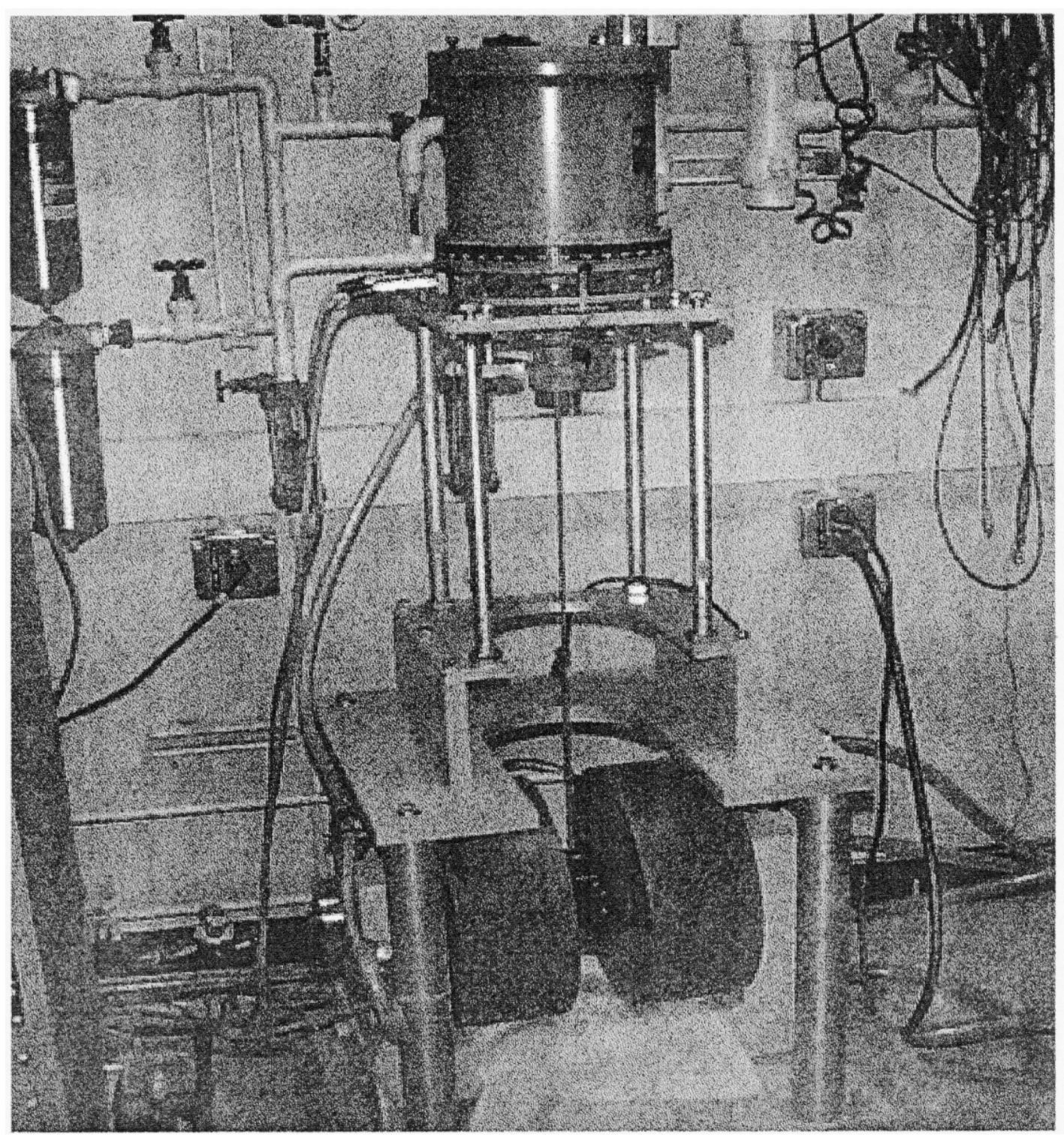

(FIG. 3) 
VSM with high temperature oven

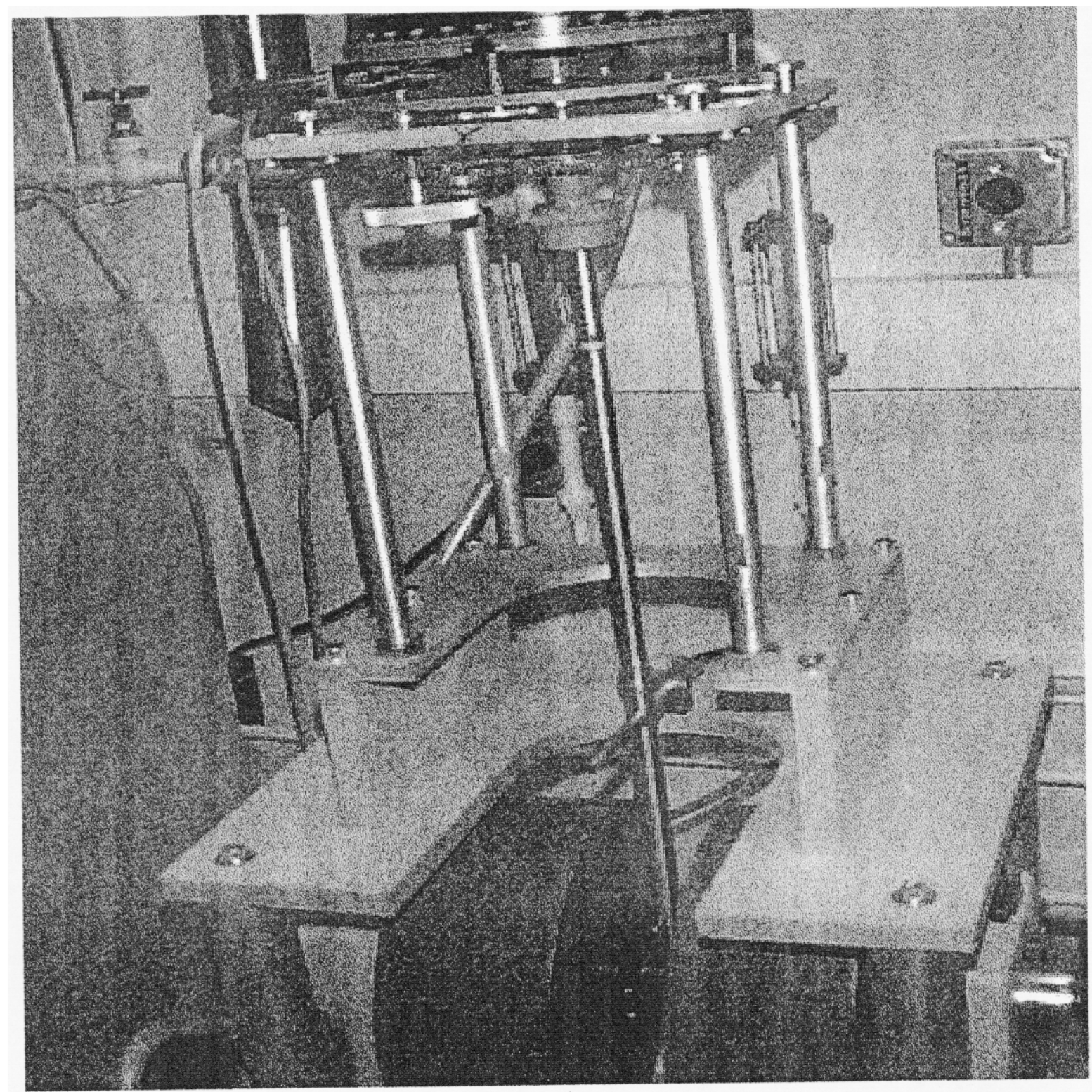

(FIG. 4) 


\section{TEMPERATURE DEPENDENT MEASUREMENTS:}

The sample investigated for temperature dependence was a bilayer consisting of a $500 \AA$ layer of $\mathrm{NiO}$ and a $50 \AA$ layer of NiFe on top of that where the $\mathrm{NiO}$ is the antiferromagnetic material and the $\mathrm{NiFe}$ is the ferromagnetic material. The ferromagnet typically wants all of its moments aligned in the same direction whereas the antiferromagnet prefers to have its moments aligned antiparallel to one another. So when the two layers are in contact there is an interaction between the two layers at the surface interface called exchange coupling. For a simplistic model the antiparallel alignment of the NiO causes the moments at the surface interface of the NiFe to align in a particular direction and since the NiFe wants all of its moments aligned in the same direction this results in the overall net magnetic moment of the NiFe being pinned in one direction. This effect is seen as a shift in the hysteresis loop of the NiFe and is referred to as the exchange field (He). There is also an increase in the coercivity $(\mathrm{Hc})$ of the NiFe due to the exchange coupling between the two layers (fig. 5).

The temperature dependent data of the bilayer at $325 \mathrm{~K}$ (fig. 6) shows this exchange field shift and an increased value of coercivity from the typical value for NiFe of $1-2$ Oe. As the temperature is increased to 500 $\mathrm{K}$ both of these affects are diminished (fig. 7). The hysteresis loop is now centered around the origin and the coercivity is clearly reduced. This occurs as the temperature approaches the Neél transition temperature for $\mathrm{NiO}(520 \mathrm{~K})$. So as this temperature is approached the antiferromagnet loses its antiferromagnetic properties and no longer interacts with the NiFe via exchange coupling. Therefore the hysteresis of the sample at the higher temperature shows the magnetic characteristics typical of a free layer of NiFe. 
Exchange Coupling between Ferromagnet / Antiferromagnet Bilayer Elements.
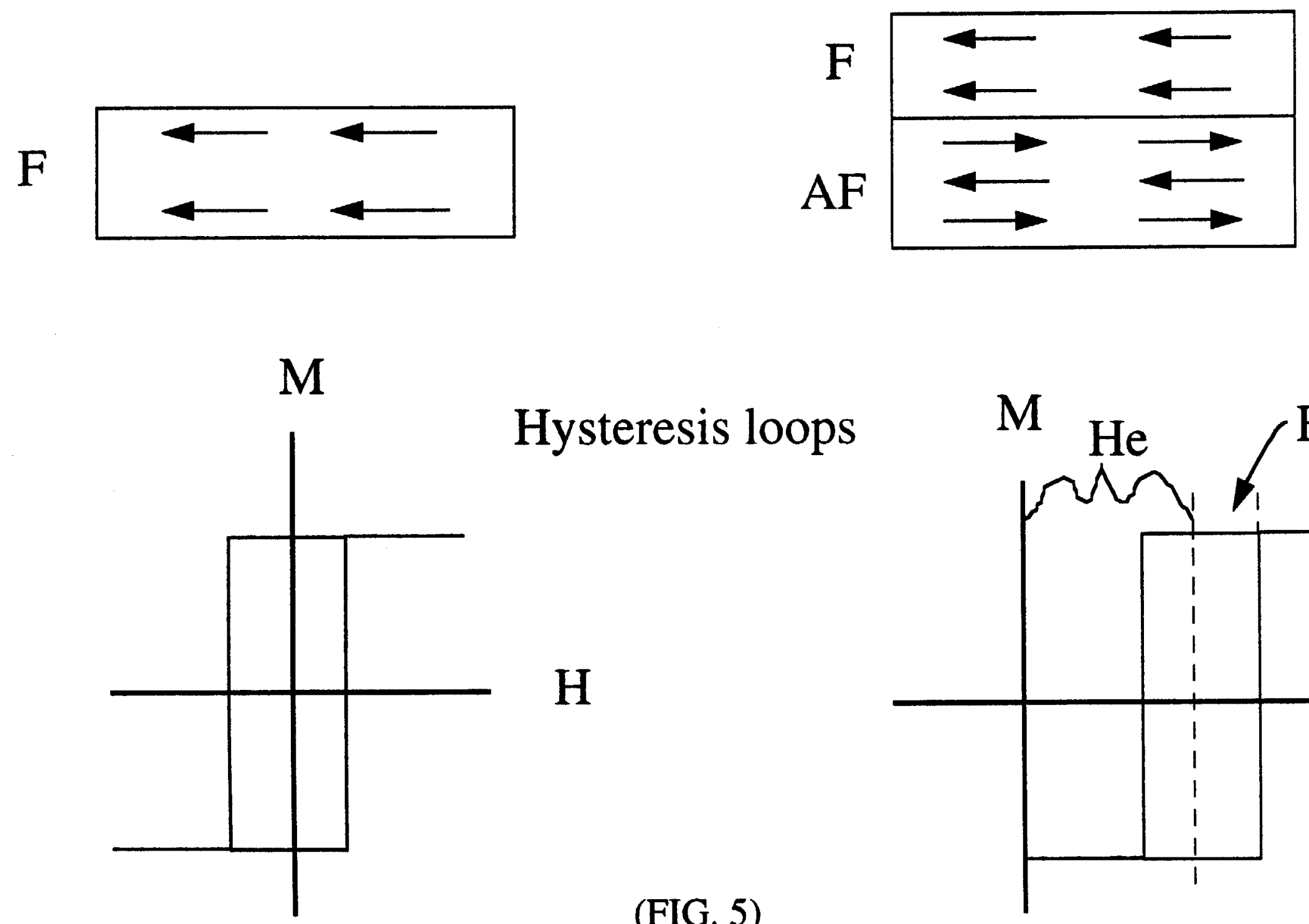

Hysteresis loops

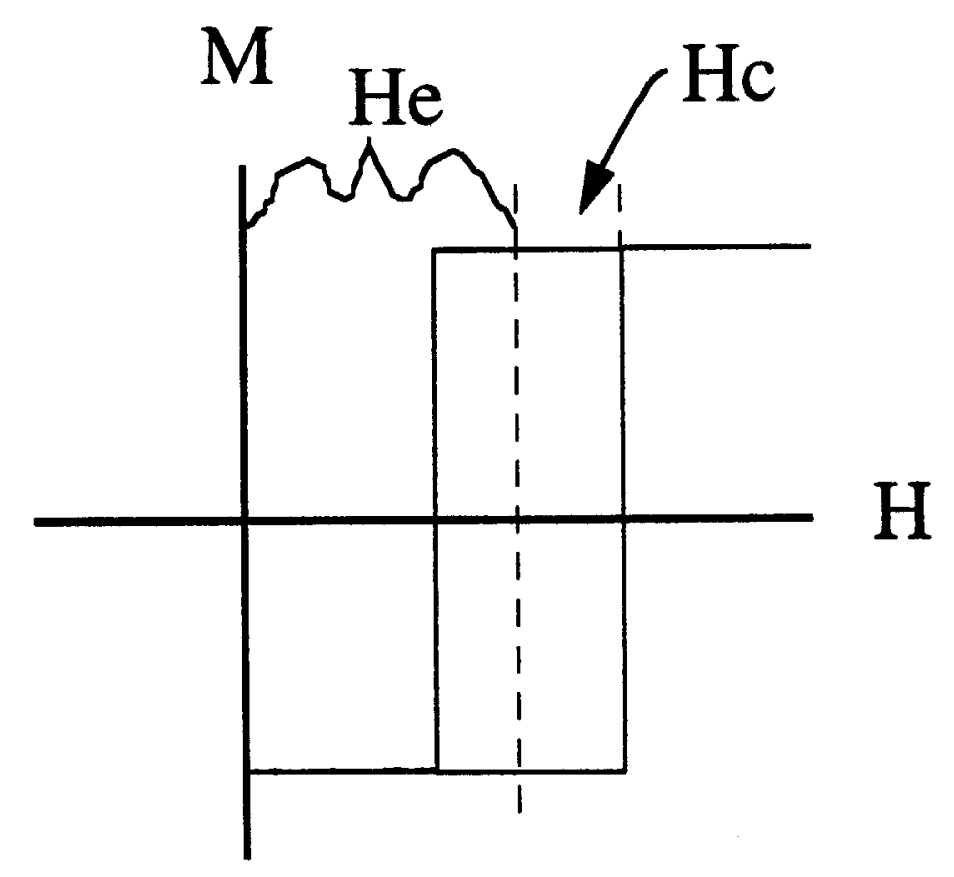

(FIG. 5) 


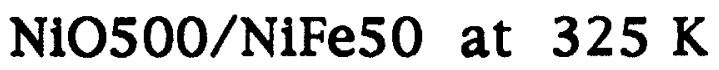

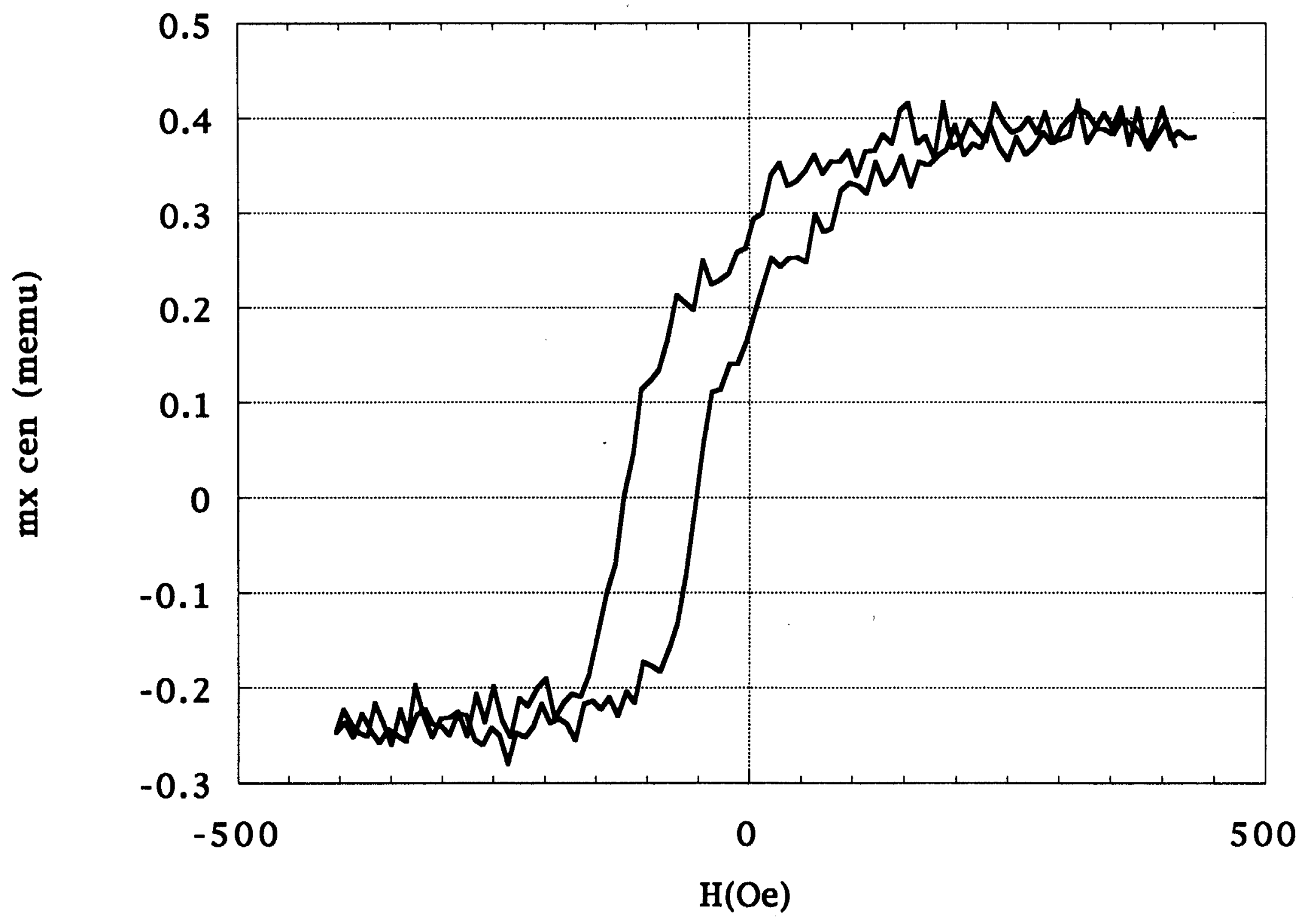

(FIG. 6) 
$\mathrm{NiO500/NiFe50}$ at $500 \mathrm{~K}$

$5 c$

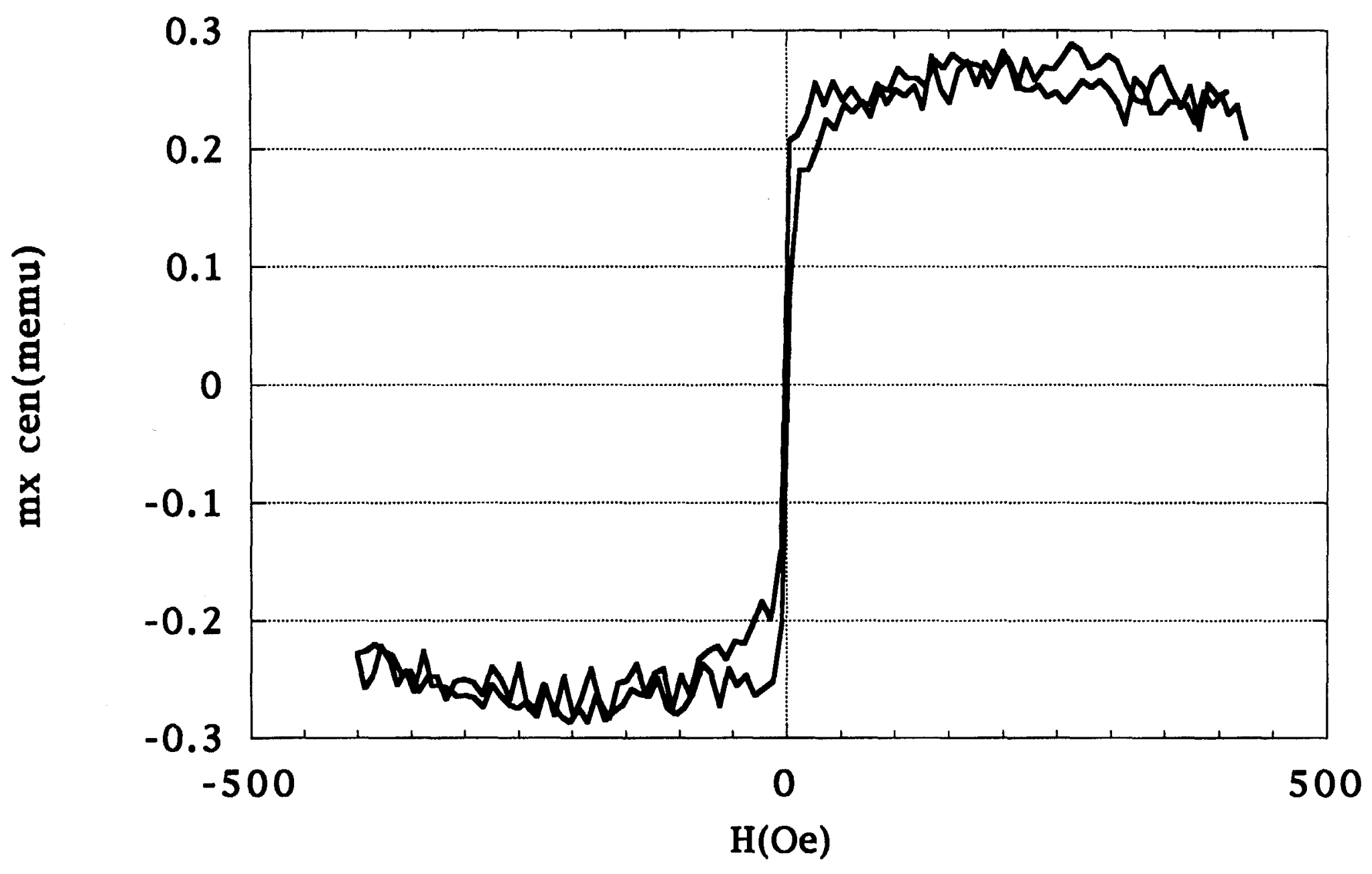

(FIG. 7) 


\section{RESULTS:}

The results of these measurements is a graph (fig. 8) which shows the He decreasing approximately linearly with increasing temperature reaching approximately zero near the transition temperature of NiO. This linear decrease occurs over the temperature range of a typical operating read head device. The coercivity also decrease with increasing temperature but at a slower rate reaching the typical value of $\mathrm{NiFe} \mathrm{(1} \mathrm{-} 2$ Oe) near the transition temperature of $\mathrm{NiO}$. Over the temperature range of a typical operating read head device the coercivity is less than the exchange field value indicating that this material would be stable for use in a read head device. If this were not the case the two ferromagnetic layers in a GMR spin-valve device would have their magnetic properties operating over the same field range meaning that their moments would not move independently of one another. This would reduce the GMR effect or possibly the device would not work at all.

\section{CONCLUSION:}

In concluding this paper: $\mathrm{NiO} / \mathrm{NiFe}$ bilayers were grown using direct ion beam sputtering of $\mathrm{NiO}$ and NiFe targets. Their magnetic properties (i.e. $\mathrm{Hc}$ and $\mathrm{He}$ ) were measured with respect to temperature in a VSM equipped with a high temperature oven. An exchange field shift and an increase in coercivity was observed due to the exchange coupling of the antiferromagnet $(\mathrm{NiO}$ ) with the ferromagnet (NiFe). The change in these properties with increasing temperature shows an approximate linear decreasing $\mathrm{He}$ for increasing temperature over the temperature range of a typical operating read head device. Also the value of Hc over this temperature range in comparison to He indicates this material to be stable for use in a GMR spin-valve device. It is clear that this temperature dependent information is an important factor to be incorporated into any future design of a GMR spin-valve read head device using this material. 
$\mathrm{Hc}$ and He vs $\mathrm{T}$ (NiO500/NiFe50)

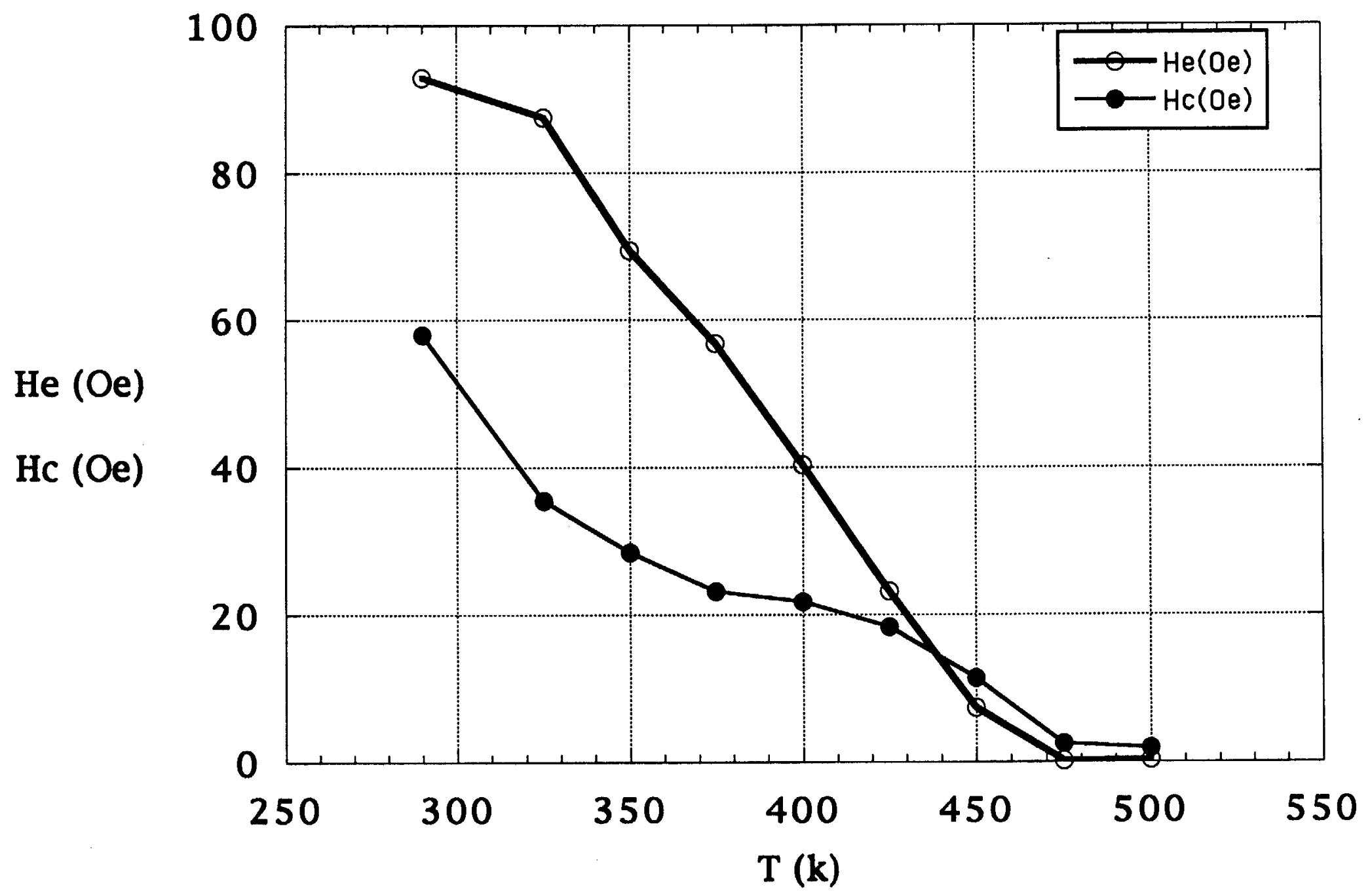

(FIG. 8) 


\section{BIBLIOGRAPHY}

I. Alison Chaiken, Richard P. Michel, Young K. Kim and Lantz E. Johnson, "NiO Exchange Bias Layers Grown by Direct Ion Beam Sputtering of a Nickel Oxide Target".

II. Katherine Derbyshire tech. ed, and Ed Korczynski ed., "Giant magnetoresistance for tomorrow's hard drives" Solid State Technology, Sept. 1995. 


\title{
A Summary of the Development of Nuclear Weapons
}

\author{
by \\ Stacy F. Lewis \\ Spelman College \\ Atlanta, Georgia
}

May 10, 1996

Edited by

Franklin D. Barish

Defense \& Nuclear Technology Directorate

Lawrence Livermore National Laboratory 


\begin{abstract}
This paper discusses the development of nuclear weapons and will be used by secondary school teachers as part of an interdisciplinary curriculum on nuclear weapon nonproliferation. This curriculum is being developed by the LLNL Education Program's Global Security Project. It is being designed to be used in high schools at the eleventh and twelfth grade level. It will expose students to scientific, mathematical, technological, and historical issues using nuclear proliferation as a complex real-world problem that necessitates knowledge in these subject areas. The material presented in this paper provides background information for teachers and will be used in lesson plans contained within the curriculum.
\end{abstract}

The material gathered for this paper came from several dozen references and is divided into two major sections:

(1) pre-WWII (1895 - 1939) and WWII (1939 -1945)

(2) post-WWII (1945 - present)

From the research conducted, it was determined that the development of nuclear weapons was most strongly influenced by :

- historical events and political confrontation

- technological advancements

- economic issues

Upon reading this paper, the teacher will have a clearer understanding of the development of nuclear weapons which will aid in instructing students on nuclear nonproliferation. 


\subsection{Requirements}

The following list is a detailed view of the requirements for the problem:

- Controls \& presentations with graphical interfaces

- Connect to an Oracle 7 (DEC/OSF UNIX) server

- Target applications accessible through desktop computers

- Functionality \& features specified by mentor(s) \& community of users (employees)

- Consistency with previous versions, especially the current version

See Figure 3.

Figure 3 presents and encapsulates a simplified view of the problem and requirements defined so far. The requirements will be clearer after the background is covered.

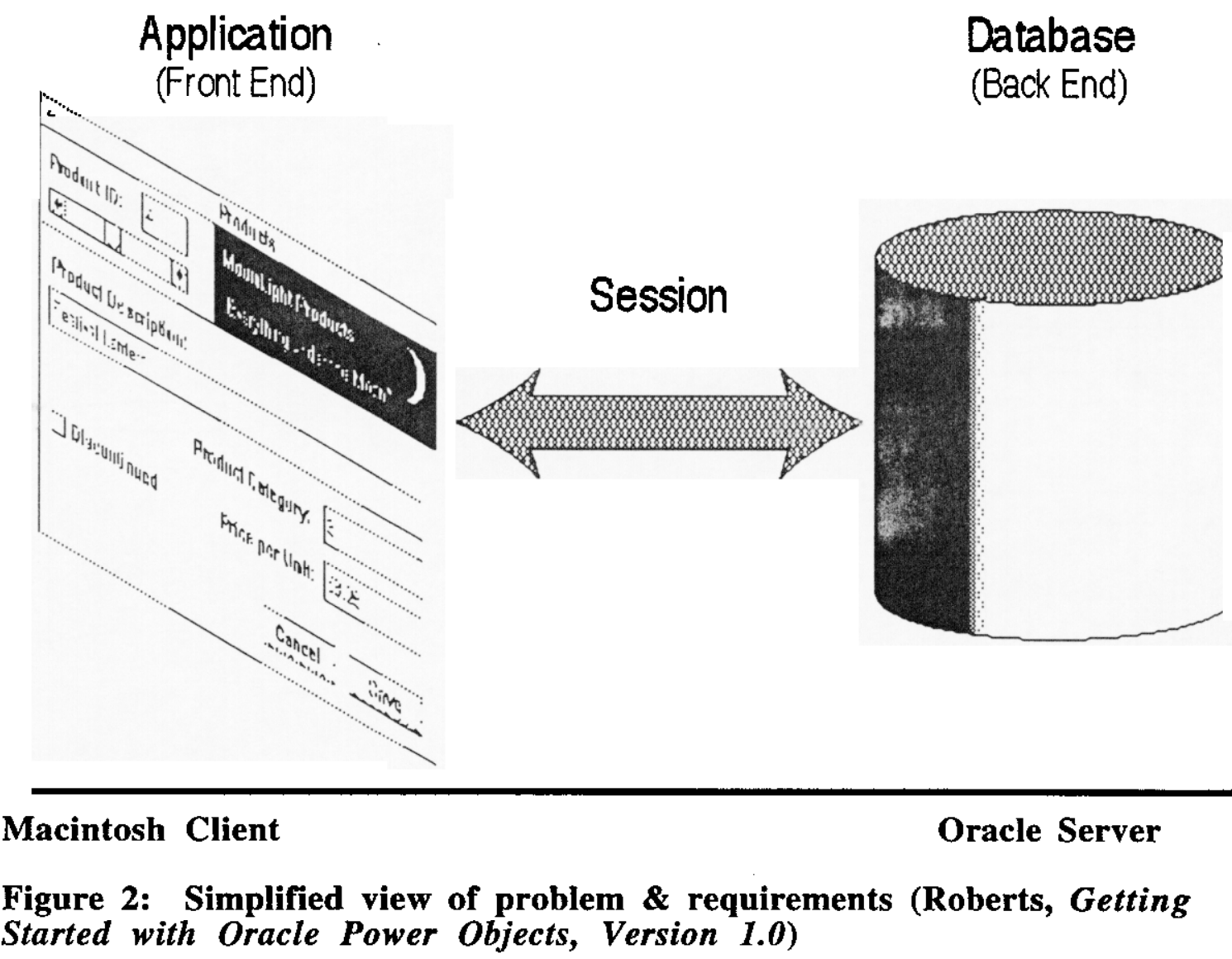




\section{Background}

\subsection{System Components}

The basic underlying components of ESIS are

[1] Data server (which holds the data and manages access to the data)

[2] Background processes, scripts, and executable programs w/ embedded SQL

(which monitor the state of the database and perform major tasks and processing)

[3] Forms (which provide 'filtered' views and front ends for component \# 2)

See Figure 3. The main focus of my project is on component \# 3: the front end of ESIS. My main objective is to provide an interface to ESIS that is easier to use than the current one.

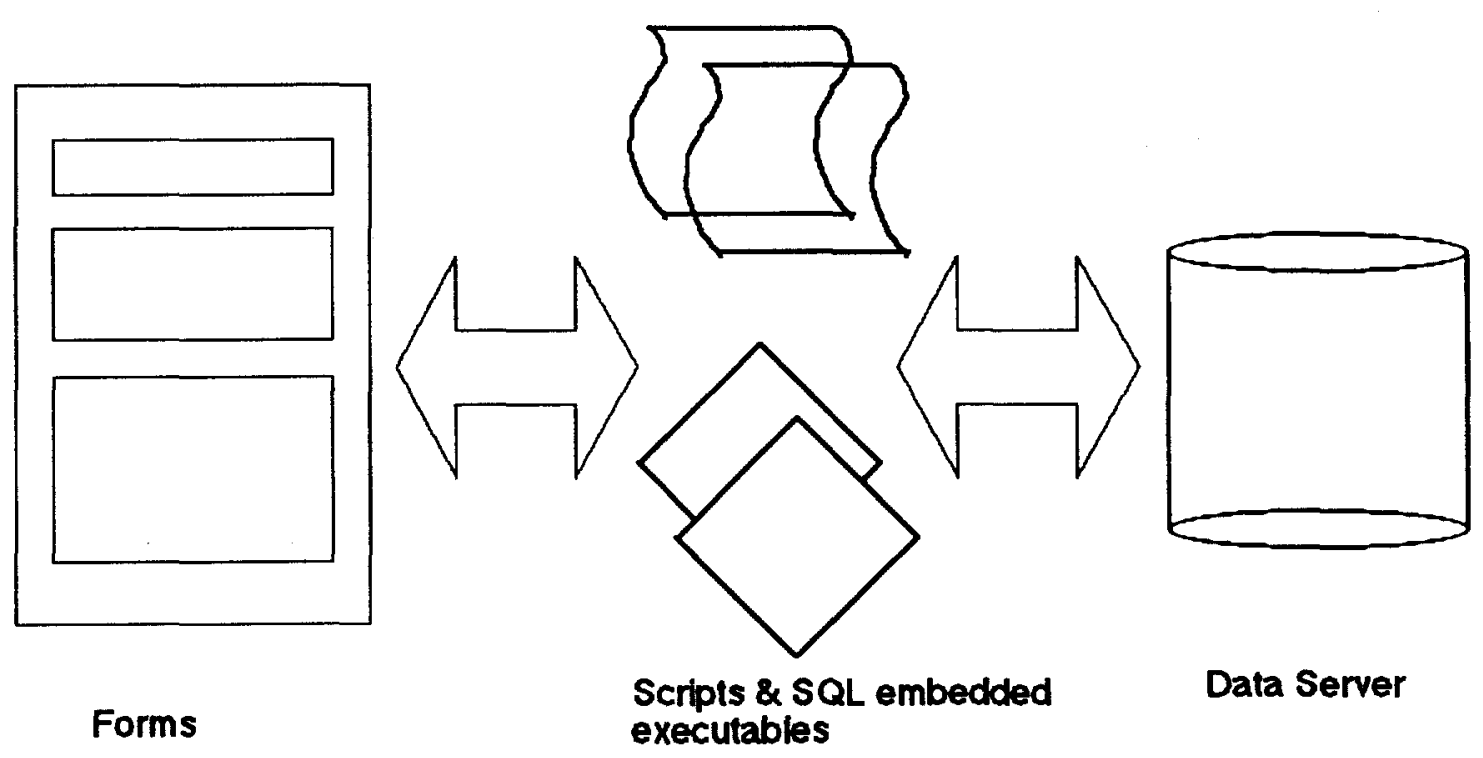

Figure 3: Basic components of ESIS 


\subsection{Business Procedures}

I In this sub-section, the business procedures -- i.e. what a typical employee

performs -- will be described. Here are the events that occur:

- Jobs and work orders are created (because an electronic component or part needs repair or service)

- Employees perform the job or work order

- Employees log hours spent performing specific work orders

- Accounts are charged for labor performed

- Employees are paid accordingly

- Work orders are completed

To log these activities, an employee must enter the data in the following electronic form: time card application. See Figure 4.

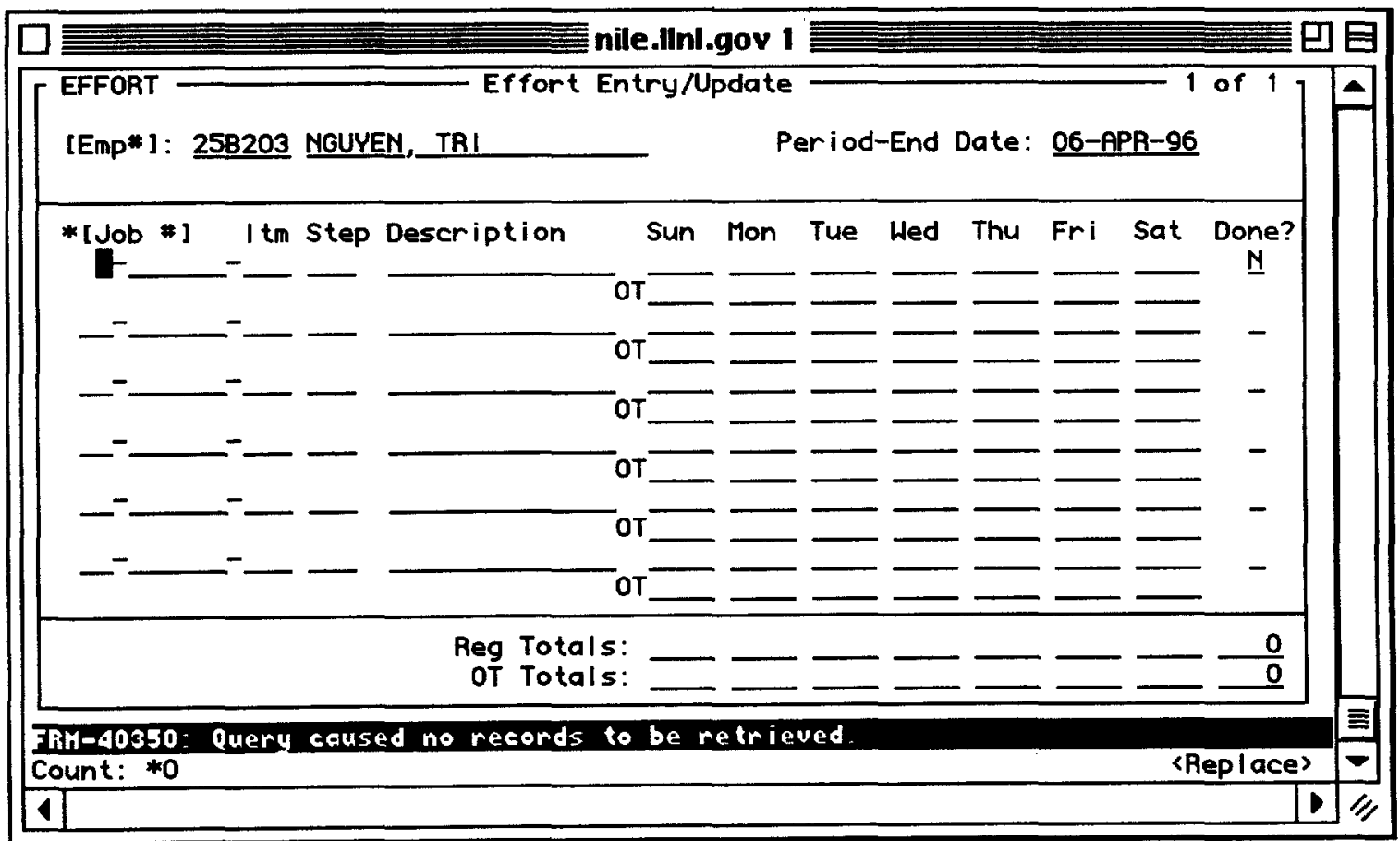

Figure 4: Time Card form with character-based interface 
The current electronic form is character-based. Evidently, the character-based presentation is not intuitive and not easy to use. To enter time, employees must navigate through fields and forms using the keyboard. Thus, employees delay time entry and logging their work. As a result, records are not updated and maintained properly.

Thus, the database would be corrupted with invalid data. 


\section{Conversion Process}

\subsection{Before Conversion}

The current interface to ESIS is a set of character-based (CB) forms. To access ESIS, a user would 'telnet' into a server machine. The user would be presented with screens as in Figures $1 \& 4$. In order to perform the tasks and transactions, the user must use the keyboard, esp. tabs, to navigate between forms and between text displays and to enter values into fields.

Figure $5 \& 6$ presents a sample of source code used to generate character-based forms like Figures $1 \& 4$. The codes for triggers and screen items associated with one form are contained in one text file. Evidently, maintenance and updates of the characterbased interfaces are difficult and tedious. 


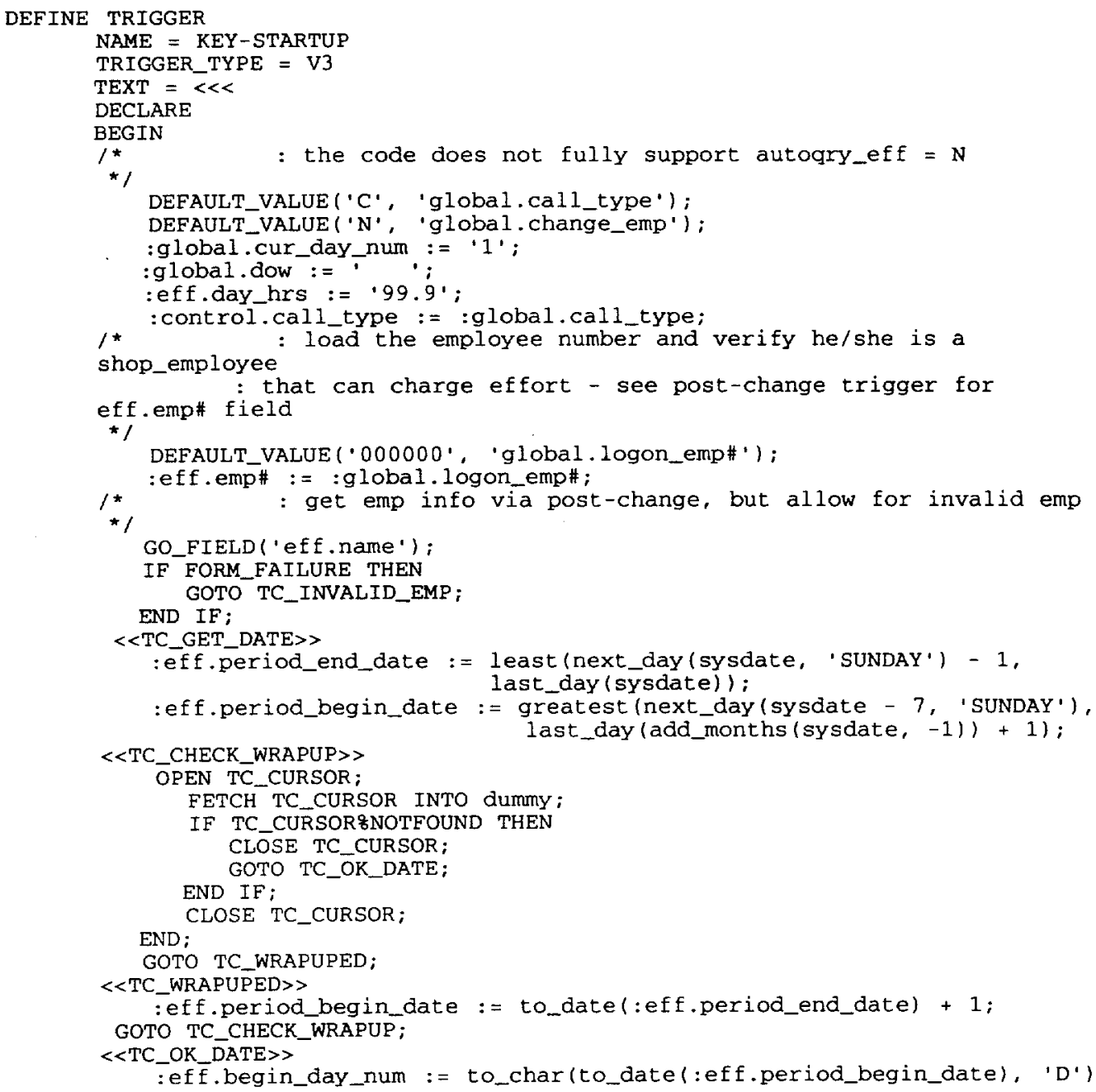

\section{Figure 5: Sample code -- trigger definition of character-based form, in particular Time Card}


The first step of the synthesis constructs the backbone of the linker molecule. It is based on methods described in the literature on organosilicon chemistry (Chu et al., 1984; Rubinsztajn et al., 1989; Patai and Rappoport, 1989). Due to the importance that no water be present in this reaction, all glassware used in this experiment was heated it to $120 \mathrm{C}$ and then cooled in an anhydrous environment. The three neck round bottom reaction flask was purged with argon gas. The necks were then fitted with rubber septa, including one with a thermometer.

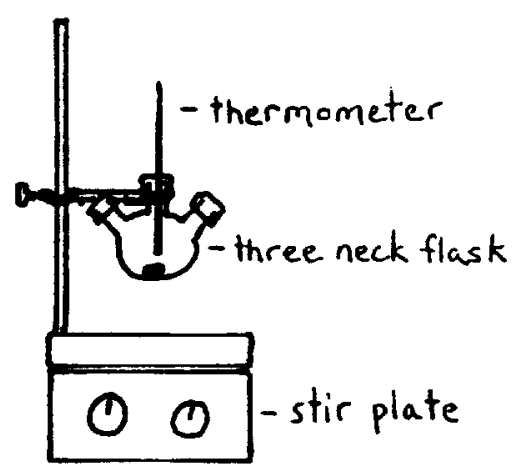

1. The apparatus was charged with exactly $0.059797 \mathrm{~mol}$ (11.6143 g) of tetraethylene glycol (TEG, Sigma Chemical Corp.) which had been stored over a $5 \dot{A}$ molecular sieve.

2. In a separate Erlenmeyer flask, $0.0724 \mathrm{~mol}(10.064 \mathrm{~mL})$ of triethylamine (Sigma Chemical Corp.) and $0.0099 \mathrm{~mol}(1.206 \mathrm{~g})$ of 4-dimethylaminopyridine (Sigma Chemical Corp.) were mixed with a spin bar.

3. Upon dissolution of the 4-dimethylaminopyridine, $222.8 \mathrm{~mL}$ of toluene (Sigma Chemical Corp.) (making a 10\% solution) and 1.21 $\mathrm{mL}$ of hexamethylphosphoramide (Sigma Chemical Corp.), both stored over a $5 \mathrm{~A}$ molecular sieve, were added to the Erlenmeyer 
make a transparent solution. This solution was then added to the round bottom flask containing the TEG.

4. Using a TEG:Si ratio of $2: 1.1,0.03289 \mathrm{~mol}(4.2459 \mathrm{~mL})$ of dichlorodimethyl silane (DDS, Sigma Chemical Corp.), was aspirated into a $5 \mathrm{~mL}$ syringe through a Teflon ${ }^{\mathrm{TM}}$ catheter. The catheter was inserted through a hole in the rubber septa on the round bottom flask and, during vigorous stirring, the DDS was slowly injected into the flask below the level of the solution.

5. Observations: Immediately, a white precipitate of triethylamine hydrochloride appeared.

6. The injection was continued at a rate that was slow enough to maintain a flask temperature below 40C. After all the DDS was added, the flask was purged with argon gas, sealed, and the mixture was allowed to stir for at least 4 hours.

7. After 4 hours had passed, the mixture was vacuum filtered through Whatman glass microfibre filters (GF/F) to remove all the triethylamine hydrochloride. The weight of the triethylamine hydrochloride after drying, amounted to $9.878 \mathrm{~g}$ (** mol). This corresponds to an apparent yield of $109 \%$.

8. The filtrate was roto-evaporated at a temperature no greater than $100 \mathrm{C}$ to leave a viscous liquid containing the crude dimethyl bis[2-[2-[2-[2-ethoxy]ethoxy] ethoxy]ethoxy] silane diol (hereinafter called the intermediate). 
Step 2: Esterification of d-Biotin to the Intermediate<smiles>CC(C)OCCOCCOCCOCCO</smiles>
intermediate

bioiin

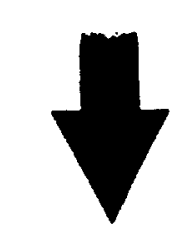

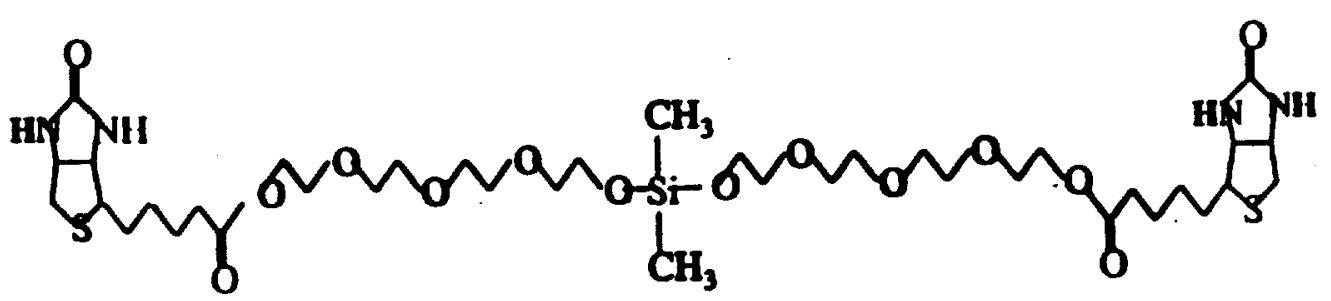
dimethyl bis $[2-[2-[2-[2-e t h o x y]$ ethoxy $]$ ethoxy]ethoxy] silane dibiotinylate

The room-temperature esterification method of Hassner and Alexanian (1978) was used as follows to attempt to esterify d-biotin to the intermediate diol. Again, all glassware used in this experiment was dried in an oven as previously described. The type and amount of solvent used in this reaction varied from reaction to reaction. Also the amounts of reagents shown are based on $1.000 \mathrm{~g}$ of d-Biotin.

1. In a $50 \mathrm{~mL}$ round-bottom flask $1 \mathrm{gram}(0.00409 \mathrm{~mol})$ of d-biotin (Sigma Chemical Corp.) was dissolved in the solvent using a magnetic stir bar. 
2. To this mixture, $0.0551 \mathrm{~g}(0.00037 \mathrm{~mol})$ 4-pyrrolidinopyridine, $0.8444 \mathrm{~g}$ (0.00409 mol) dicyclohexylcarbodiimide (DCC), and $0.8272 \mathrm{~g}(0.00186 \mathrm{~mol})$ of dimethyl bis [2-[2-[2-[2ethoxy]ethoxy]ethoxy] ethoxy] silane diol were added. The mixture was set to stir overnight. The outcome of the reaction varied depending on the solvent.

\section{Results}

The first step was successful in that it resulted in the expected triethylamine hydrochloride precipitate at approximately $109 \%$ yield. The second step esterification reaction had varied results. Refer to the following table for the details of the esterification reaction:

Table 1: Esterification Reaction Observations.

* The following solvents do not dissolve one or more of the reagents in the 2 nd step:

\begin{tabular}{lll} 
Date & Solvent & Amount \\
\hline $3 / 19,4 / 11$ & methylene chloride & $20.00 \mathrm{~mL}$ \\
$4 / 18$ & ether & $25.00 \mathrm{~mL}$ \\
$4 / 4$ & intermediate & $0.8444 \mathrm{~g}$ \\
$4 / 10$ & toluene & $20.00 \mathrm{~mL}$ \\
$4 / 12$ & dimethyl formamide & $5.00 \mathrm{~mL}$ \\
$4 / 10$ & acetone & $20.00 \mathrm{~mL}$
\end{tabular}

the following solvents dissolve the reagents, but do not yield the desired precipitous product:

$\begin{array}{lll}4 / 12 & \text { dimethyl sulfoxide } & 20.00 \mathrm{~mL} \\ 4 / 29 & \text { pyridine } & 130.00 \mathrm{~mL}\end{array}$

The first group of solvents did not yield a reaction due to the fact that the reagents were not dissolving in the solvents. The reagents did dissolve in the second group of solvents. The fact that the dimethyl sulfoxide and the pyridine reactions did not yield a urea precipitate can be explained by one of two things. Either the reaction did not occur or the urea is soluble in these solvents. 


\section{Conclusions}

The percent yield of the triethylamine hydrochloride in the first step is an indication that the intermediate is formed.

* The absence of a urea precipitate in the second step is an indication of one of two things:

- the product is not formed

- the precipitate is soluble in the solvent

Future efforts will be to first determine if the product is formed in the second step by making urea and testing to see if it dissolves in the pyridine. If the urea does not dissolve in the pyridine, this is shown that the esterification reaction was unsuccessful and urea was not produced. However, if the urea does dissolve in pyridine, this is an indication that it is possible that the esterification reaction did occur and our product was formed. If this is the case, then the crude product will be tested for functionality.

If it is shown that the product was not formed in the second step we will attempt to and alternate synthesis. This synthesis will reverse the order of reactions such that the TEG is biotinylated before reacting it with dichlorodimethyl silane. 


\section{References}

Chu, H.K., M.D. Johnson, and C.L. Frye. 1984. Tertiary alcoholysis of chlorosilanes via tetracoordinate silylated quaternary ammonium intermediates. J. Organometaqlic Chem. 271, 327-336.

Hassner A. and V. Alexanian. 1978. Direct room temperature esterification of carboxylic acids. Tetrahedron Letters, 46, 44754478.

Patai, S. and Z. Rappoport, eds. 1989. The Chemistry of Organic Silicon Compounds, Part 1. John Wiley \& Sons, New York, NY.

Rubinsztajn, S., M. Cypryk, and J. Chojnowski. 1989. Condensation of model linear siloxane oligomers possessing silanol and silyl chloride end groups. The mechanism of silanol silyation by a chlorosilane in the presence of neutral nucleophiles. $J$. Organometalic Chem. 367, 27-37. 


\title{
Viscoelastic Properties of Healthy Human Artery Measured in Saline Solution by AFM-Based Indentation Technique
}

\author{
E. Lilleodden*, A. Lundkvist**, W. Siekhaus*, J. Kinney* and M. Balooch* \\ * Lawrence Livermore National Laboratory, Livermore CA 94550 \\ ** University of California, Berkeley CA 94720
}

\begin{abstract}
Using an Atomic Force Microscope with an attachment for indentation, we have measured local, in vitro mechanical properties of healthy femoral artery tissue held in saline solution. The elastic modulus $(34.3 \mathrm{kPa})$ and viscoelastic response $\left(\tau_{\varepsilon}=16.9 \mathrm{~s}\right.$ and $\left.\tau_{\sigma}=29.3 \mathrm{~s}\right)$ of the unstretched, intimal vessel wall have been determined using Sneddon theory and a three element model (standard linear solid) for viscoelastic materials. The procedures necessary to employ the indenting attachment to detect elastic moduli in the $\mathrm{kPa}$ range in liquid are described.
\end{abstract}

\section{INTRODUCTION}

Coronary artery disease or atherosclerosis is the leading cause of death in the United States [1]. Percutaneous Transluminal Coronary Angioplasty (PTCA) is the most common treatment for patients suffering from atherosclerosis. Although PTCA yields a greater than $90 \%$ immediate success rate, the $30-40 \%$ restenosis rate, or re-narrowing of the coronary arteries, hinders complete success [2]. A significant portion of this restenosis rate is due to geometric remodeling of the diseased artery wall incurred during balloon inflation (e.g. medial stretching [2], plaque dissection [3] and plaque compression [2,3]). If the plaque contains a significant amount of calcium deposits, preferential regions of high stress conducive to stretching or dissection may form during balloon inflation. Eighty percent of patients recommended for PTCA exhibit calcium deposits in the plaque [4]. Hence, there is particular clinical interest in understanding the local material properties and mechanical behavior of calcified atherosclerotic deposits and healthy arterial wall.

The present work describes the technique of using the AFM-based nanoindenter to determine the mechanical properties of soft tissues held in saline solution. The static elastic modulus and viscoelastic constants of the intimal surface of a healthy human femoral artery are reported. These results will be used as a base for further studies on atherosclerotic coronary tissues.

\section{EXPERIMENTAL TECHNIQUE AND RESULTS}

The Atomic Force Microscope (small stage Nanoscope III, Digital Instruments, Santa Barbara, CA) has been modified by replacing the conventional head assembly with a transducerindenter assembly called a Micromechanical Testing Instrument (Hysitron Inc., Minneapolis, MN). Like its conventional counterpart, the instrument can image the topography of specimens by tracing the superficial contours of the sample with nano-Newton loads. In addition, the device is a force-generating and depth-sensing instrument (Nano-indenter) capable of generating loaddisplacement curves at specific locations in ambient and, with the corrections described here, liquid environments. The minimum load applied is less than $1 \mu \mathrm{N}$ and the maximum displacement measured is $35 \mu \mathrm{m}$. Indenters of varying materials (diamond, tungsten carbide, aluminum); shapes (Berkovich, spherical, flat cylindrical punch) and a range of diameters (.17 to $2.0 \mathrm{~mm}$ ) have been used for indentation of healthy human arteries.

For the experiments reported here, healthy femoral arteries supplied by the National Disease Research Interchange (NDRI) were scanned by intravascular ultrasound (Boston Scientific, Sunnyvale, CA) to ascertain that no plaque existed. The vessel was cut longitudinally, sectioned in $5 \mathrm{~mm} \times 5 \mathrm{~mm}$ regions and placed unstretched in a .9\% saline bath on the AFM stage.

The basic components of the Micromechanical Testing Instrument are shown in Figure 1. The force recorded and displayed by the instrument is defined as, 
$F_{\text {display }}=A \varepsilon_{0} \frac{v^{2}}{d_{0}^{2}}$

where $A$ is the plate area, $V$ is the voltage applied between the drive plate and pickup electrode, $\varepsilon_{0}$ is the dielectric constant and $\mathrm{d}_{0}$ is the spacing between the pickup electrode and drive plate when no weight (such as the indenter) is attached and no voltage is applied. The vender specifies $d_{0}$ as $120 \mu \mathrm{m}$. In many material science applications, equation (1) adequately represents the actual force applied to the material under investigation.

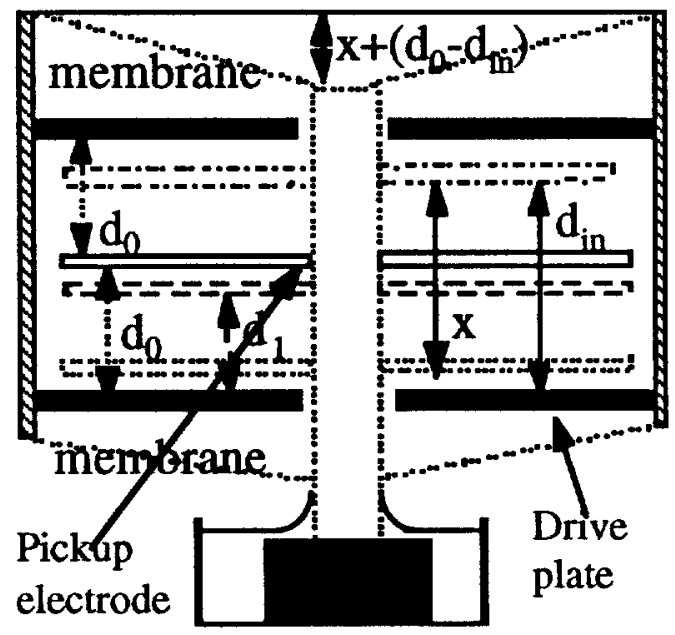

Figure 1. Schematic of Micromechanical Testing Instrument with parameters needed to determine the actual force applied to soft materials in saline solution. The combination of pickup electrode and drive plates is used to both generate a force, $F_{\text {display, }}$ and to measure displacement, $\mathbf{x}$.

For soft materials, however, the displacements could be large even for small applied voltages. In this case, the change in the plate spacing deviates substantially from that described by equation 1 , and the force displayed does not equal the force actually applied to the sample. Additionally, the stiffness of the membrane, the weight of the indenter itself and the force exerted on the indenter's shaft as a result of surface tension should be included to accurately describe the applied force in liquid. The actual force applied to the sample is

$F_{a}=F_{\text {display }}\left[\frac{d_{0}}{d_{\text {in }}-x}\right]^{2}-K_{s}\left(x+d_{0}-d_{\text {in }}\right)+F_{\text {s.t. }}+M g$

where $x$ is the displacement of the pickup electrode from its initial position $d_{i n}, K_{s}$ is the spring constant of the membrane (ignoring possible nonlinearity), $\mathrm{Mg}$ is the weight of the indenter and $F_{\text {s.t. }}$ is the force due to surface tension. When a preload is applied to the sample, the force due to the initial displacement is

Preload $=K_{s}\left(d_{\text {in }}-d_{1}^{\text {liq }}\right)$

In the absence of an initial preload, the initial spacing, $d_{i n}$, is replaced by 
$\mathbf{d}_{1}^{\text {liq }}=\mathbf{d}_{1}^{\text {air }}-\frac{F_{\text {s.t. }}}{\mathbf{K}_{s}}$

where $d_{1}{ }^{\text {air }}$ is the spacing between the pickup electrode and drive plate in air due to the weight of the attached indenter (Figure 1). $\mathrm{K}_{\mathrm{s}}$ and $\mathrm{d}_{1}{ }^{\text {air }}$ are determined by making a best fit to displayed force versus distance curves of several calibration runs done in air without a load applied to the sample. The results are shown in Figure 2a along with the deviation of experimental points from the optimum fit. The experiments were repeated in liquid with no load applied to the sample to obtain $\mathrm{F}_{\text {s.t. }}$. The result of the curve fit and its deviation from data points in liquid is shown in Figure 2b.

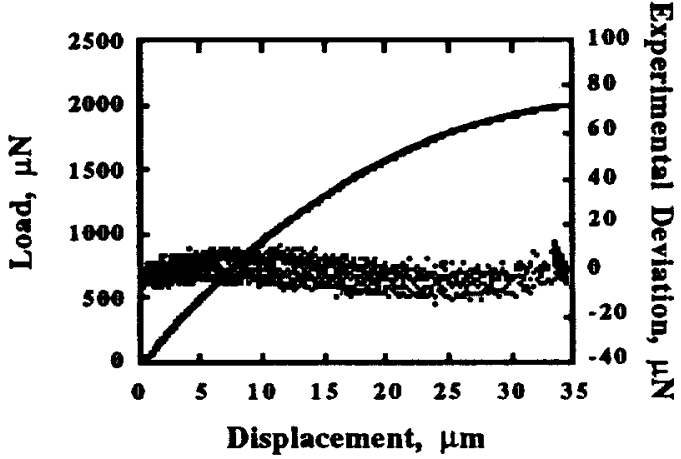

$2 a$

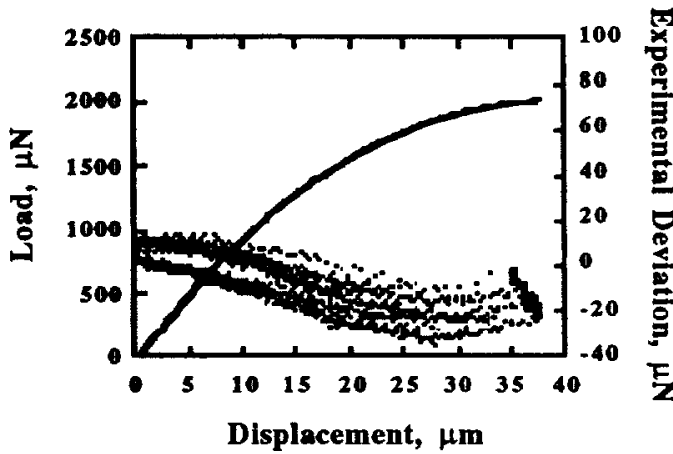

$2 b$

Figure 2. Displayed force vs. displacement with no load applied to the sample, and the deviation of experimental results from the optimum fit to force vs. displacement: a) in air b) in saline solution.

Since healthy human arteries are known to have high compliance, this study used a flat circular punch consisting of a thin aluminum disk $2 \mathrm{~mm}$ in diameter, attached to a stem of $.5 \mathrm{~mm}$ diameter (to minimize surface tension forces) held by the mechanical testing instrument. The "sine wave" appearance of the deviation of experimental results from the optimum fit to curves of force vs. displacement evident in both air and liquid calibration runs in Figure 2 can be eliminated when a nonlinear third-power term is added, as suggested by Timoshenko [5], to describe the force vs. displacement of the membrane. The load accuracy of the measurements is $\pm 10 \mu \mathrm{N}$ in air and $\pm 20 \mu \mathrm{N}$ in saline solution, and by almost a factor of two better when a nonlinear spring is considered.

The data were analyzed using a simple three element model (standard linear solid [6])--a spring $\left(\mu_{0}\right)$ placed in parallel with a Maxwell spring $\left(\mu_{1}\right)$-damper $\left(\eta_{1}\right)$ system. The behavior of this model is characterized by three parameters:

$\tau_{\varepsilon}=\frac{\eta_{1}}{\mu_{1}}, \quad \tau_{\sigma}=\frac{\eta_{1}}{\mu_{0}}\left(1+\frac{\mu_{0}}{\mu_{1}}\right) \quad$ and $\quad E_{R}=\frac{\mu_{0}}{2 \mathrm{r}}$

where $\tau_{\varepsilon}$ is the relaxation time for constant strain, $\tau_{\sigma}$ is the relaxation time for constant stress, $r$ is the indenter radius and $E_{R}$ is the relaxed elastic modulus of the system as time approaches infinity, based on contact stiffness and Sneddon indentation theory $[7,8]$. The creep response [6], $c(t)$, of this system to a constant force of magnitude unity, $u(t)$, is described as

$c(t)=\frac{1}{\mu_{0}}\left[1-\left(1-\frac{\tau_{\varepsilon}}{\tau_{\sigma}}\right) e^{-t / \tau_{0}}\right] u(t)$ 
The actual force and displacement vs. time for a healthy artery are shown in Figure 3 . The instrument was programmed to generate a trapezoidal "display-force" vs. time. Applying the corrections described in equations (2) - (4) provided the time-dependent actual applied force shown in Figure 3. A second order polynomial was fit to the loading portion of the actual applied force vs. time curve and this fitted analytic function, $F(t)$, was integrated using the Boltzmann superposition principle for creep response given an arbitrary load profile [6]. This results in an analytic displacement function, $X(t)$, containing three undetermined model parameters $\mu_{0}, \tau_{\varepsilon}$ and $\tau_{0}$.

$$
\mathbf{X}(\mathbf{t})=\frac{1}{\mu_{0}}\left[\left.\mathbf{F}(\mathbf{t})\right|_{0} ^{\mathbf{t}}-\left(1-\frac{\tau_{\varepsilon}}{\tau_{\sigma}}\right) \mathrm{e}^{-t / \tau_{\sigma}} \int_{0}^{t} \mathrm{e}^{+\tau / \tau_{\sigma}} \frac{\mathbf{d F}(\tau)}{\mathbf{d} \tau} \mathrm{d} \tau\right]
$$

This function was then fit to the measured displacement during the loading-only portion of the displacement vs. time data to determine the model parameters. These parameters were then used in the Boltzmann integral on the actual applied load curve $F(t)$ to predict the displacement vs. time data, as plotted in figure 3.

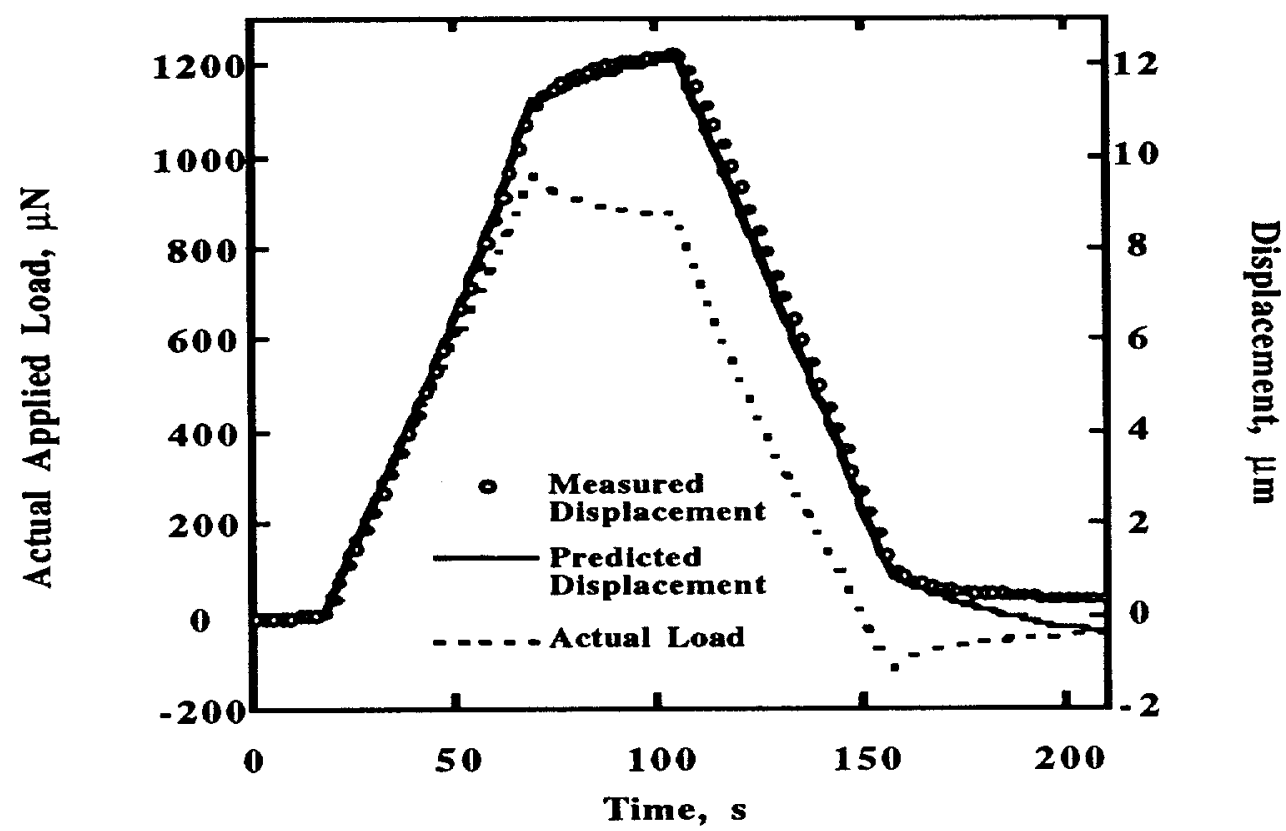

Figure 3. The actual force applied and measured displacement as a function of time for a healthy, femoral artery wall using a $2 \mathrm{~mm}$ diameter, aluminum, flat punch. The Boltzmann superposition principle was used to predict the displacement vs. time based on the Kelvin model parameters and the time-dependent loading curve.

Table I compares the results of this study with previous indentation studies. Although the elastic modulus values reported by Gow et al. $[9,10]$ were obtained with indenters smaller in diameter than used here, information about the viscoelastic response is not provided. The technique used by Lee et al. [11] used a $7 \mathrm{~mm}$ diameter indenter, and hence the results represent an average over an area mone than 10 times larger than the area used in this study. In addition to providing local measurements of the elastic modulus of healthy, femoral artery (intimal) wall, to the best of the authors' knowledge, this investigation represents the first in vitro, localized assessment of the viscoelastic response of healthy inner arterial wall. 
Table I: Comparison of Previous Indentation Studies with this Study (*)

Aortic Intimal

Surface $[9,10]$

Elastic

Modulus (kPa)

$90-152$

$\tau_{\varepsilon} \quad(\sec )$

$\tau_{\sigma}$

(sec)
Non-Fibrous Aortic

Plaque Caps [11]

$41.2 \pm 18.8$

34.3

16.9

29.3

\section{SUMMARY AND CONCLUSIONS}

The add-on transducer to the standard AFM enhanced the capability of the instrument to measure local mechanical properties in addition to topographic imaging. To further increase its capability for use on soft tissues held in aqueous solution one also needs to consider the forces applied to the indenter, such as weight and surface tension, that are usually negligible for most materials used in engineering applications. By considering these forces, the viscoelastic properties of an unstretched healthy artery wall in saline solution have been measured. To the authors' knowledge, there is no previous 3 parameter based model of the mechanical response of the intimal layer of the artery wall. The instrumentation described here is being used with smaller indenters (.175 mm diameter) to measure the static mechanical and viscoelastic response of healthy and diseased arteries over a wide range of frequencies. These investigations will provide a sound engineering basis for future balloon angioplasty studies, and will help improve the design of other clinical devices used to treat patients with coronary artery disease.

\section{ACKNOWLEDGMENTS}

This work was supported by the U.S. Department of Energy, through the LDRD program at Lawrence Livermore National Laboratory under contract No. W-7405-ENG-48.

We want to thank Dr. W. Gerberich, U. of Minnesota, Dept. of Mechanical Engineering and Material Science; Dr. P. Yock, Stanford University Medical Center for Research in Interventional Cardiology; Greg Hyde, Guidant Corp., Advanced Cardiovascular Systems; and Dr. Wayne Bonin, Hysitron Inc. for their contributions to the scientific and technical substance of this paper. 


\section{REFERENCES}

1. Heart and Stroke Facts: 1994 Statistical Supplement, American Heart Association.

2. R. Virmani, A. Farb and A.P. Burke, American Heart Journal 127: 163-179 (1994).

3. B.F. Waller, C.M. Orr, C.A. Pinkerton, J. Van Tassel, T. Peters and J.D. Slack, Journal of the American College of Cardiology 20 (3): 701-706 (1992).

4. L.A. Fitzpatrick, A. Severson, W.D. Edwards and R.T. Ingram, Journal of Clinical Investigation 94: 1597-1604 (1994).

5. S. P. Timoshenko and J. N. Goodier. Theory of Elasticity, 3rd edition. (McGraw Hill Book Company, New York, 1970).

6. Y.C. Fung, Biomechanics, 2nd ed. (Springer-Verlag New York, Inc., New York, 1993), pp.41-46.

7. I.N. Sneddon, International Journal of Engineering Science 3: 47 (1965).

8. G.M Pharr, W.C. Oliver and F.R. Brotzen, Journal of Materials Research 7 (3): 613617 (1992).

9. B.S. Gow and R.N. Vaishnav, Journal of Applied Physiology 38 (2): 344-50 (1975).

10. B.S. Gow, W.D. Castle and M.J. Legg, Journal of Biomechanics 16 (6): 451-458 (1983).

11. R.T. Lee, G. Richardson, H.M. Loree, A.J. Grodzinsky, S.A. Gharib, F.J. Schoen and N. Pandian, Arteriosclerosis and Thrombosis 12 (1): 1-5 (1992). 


\title{
Retardation and Diffusion of VOCs in Ground Water Brian Manz / Fred Hoffiman Environmental Restoration Division Lawrence Livermore National Laboratory
}

\section{May 9, 1996}

* This research was supported in part by an appointment to the U.S.

Department of Energy Science and Engineering Research Semester (SERS). This program is administrated by Lawrence Livermore National Laboratory (LLNL) under Contract W-7405-Eng-48.

\begin{abstract}
The aquifers beneath the LLNL site have been contaminated by volatile organic compounds (VOCs) since World War II. Presently, redmediation is underway to prevent the spread of the contaminants into nearby municipal wells. The current "pump and treat" method seems to have control over the plume but will require decades to completely remove the contamination.

The objective of this research is to develop a better understanding of the physical and chemical processes retarding the VOCs and impeding the cleanup. This study will determine if velocity differences of simulated cleanups affect the degree of retardation, establish effective diffusion coefficients, and establish coefficients of retardation for different VOCs and different aquifer materials.
\end{abstract}




\section{Introduction}

The aquifers beneath the LLNL site are comprised mostly of unconsolidated alluvial sediments containing very small amounts of organic carbon $(<0.1 \%)$. There has been sufficient research to suggest that in aquifers containing $>0.1 \%$ organic carbon, absorption of VOCs into the organic material is the dominant mechanism of retardation (Piwoni and Banerjee, 1989). In low organic carbon aquifers however, very little research has been conducted to determine how the VOCs are retarded.

As contaminants move through an aquifer, they are retarded by two processes. Those controlled by adsorption and those controlled by diffusion. Adsorption of VOCs onto sand grains is assumed to be instaneous and equilibrium is maintained throughout (Fetter, 1992). Diffusion of VOCs into immobile water however, is very slow with respect to the velocity of the water (Fetter, 1992). Adsorption is reversible and therefore can be remediated easily. However, diffusion is extremely difficult to remediate due to the long periods of time required to allow for diffusion out of immobile water. It is diffusion that is the controlling mechanism responsible for the long times required for ground water cleanup. Our goal is to perform a series of column and diffusion experiments in the laboratory to improve our understand of retardation and allow for new strategies of rapid ground water cleanup.

To date, we have completed two column experiments $(4 \mathrm{ml} / \mathrm{hr} \& 8 \mathrm{ml} / \mathrm{hr}$ ) and one diffusion experiment. From our first column experiments, we discovered a colloidal substance present in the effluent from the freshwater cleanup. Upon further investigation, this substance was discovered to be illite clay. We are currently running two simultaneous column experiments, one with the illite removed (Non-Illite) and one with it still present (Illite).

\section{Methods}

In both experiments, column and diffusion, a very clean ( $0 \%$ organics) fine grained sand (Oklahoma \#1) was used to mimic the aquifer conditions at the LLNL site. Both experiments were conducted using four VOCs also present in the contaminated ground water on site: Trichloroethlyene (TCE), Perchloroethylene (PCE), Carbon Tetrachloride $\left(\mathrm{CCl}_{4}\right)$, and Chloroform $\left(\mathrm{CHCl}_{3}\right)$. VOC solutions were prepared to simulate the concentration levels in the ground water on site (250 ppb). 


\section{Column Experiment (Figure I)}

In this classic experiment, a glass / teflon column was packed with Oklahoma \#1 sand and saturated with deionized water. The contaminated water, also containing chloride (dissolved as $\mathrm{NaCl}$ ) as a tracer, was then passed through the column and effluent samples were collected to establish the breakthrough curve. As soon as the effluent concentration reached the same level as the influent, the contaminated water was replaced with deionized water to simulate a ground water cleanup. Two column experiments were run at different velocities $(4 \mathrm{ml} / \mathrm{hr} \& 8 \mathrm{ml} / \mathrm{hr})$ to record any retardation differences.

\section{Packing procedure}

All columns were packed by slowly pouring $O K$ \#1 sand into a water filled column under vibration.

NOTE: The column experiments we are currently running were packed using $\mathrm{CO}_{2}$ to minimize air bubbles in the pore space. The $\mathrm{CO}_{2}$ was filled into the beaker containing the pre-weighed sand and bubbled through the DI water prior to pouring. By using this method, we replace all of the air with $\mathrm{CO}_{2}$ which is soluble in water .

\section{Procedure for Tlite removal}

Illite clay was removed from the OK \#1 through a series of flushes with .1M $\mathrm{NaCl}$ followed by ultrasound and rinsed with DI water.

\section{Diffusion Experiment (Figure II)}

In this experiment, twenty vials were filled with Oklahoma \#1 sand, saturated with DI water, and placed into the diffusion apparatus. The contaminated water was then passed over the vials at a very low velocity $(0.5 \mathrm{ml} / \mathrm{hr})$. A glass plate was placed over the apparatus to minimize volatilization. By using this method, the only way contaminants can enter the sand filled vials is by diffusion. After 13 days the vials were removed, crushed, and analyzed for VOCs.

\section{Packing Procedure (Figure III)}

Vials were packed by pouring $O K$ \#1 sand into water filled vials under vibration. All vials were vibrated for a total of six minutes 


\section{Bulk Density, Porosity and Hydraulic Conductivity}

Calculations of bulk density and porosity were made gravimetrically for the vials and columns. Hydraulic conductivity was measured through the use of a constant head Mario bottle.

Porosity $=V_{\text {pores }} / V_{\text {total }}=\mathbf{. 3 2}$

Bulk Density $=M_{\text {solids }} / V_{\text {total }}=1.77 \mathrm{~g} / \mathrm{cm}^{3}$

Hydraulic Conductivity $(K)=Q / \mathrm{QA}=4.01 \times 10^{-3} \mathrm{~cm} / \mathrm{s}$

NOTE: These calculations were performed on three separately packed columns and twenty separately packed glass vials. In each case, the values for bulk density, porosity, and hydraulic conductivity were all the same.

\section{Column Experiment (Figure I)}

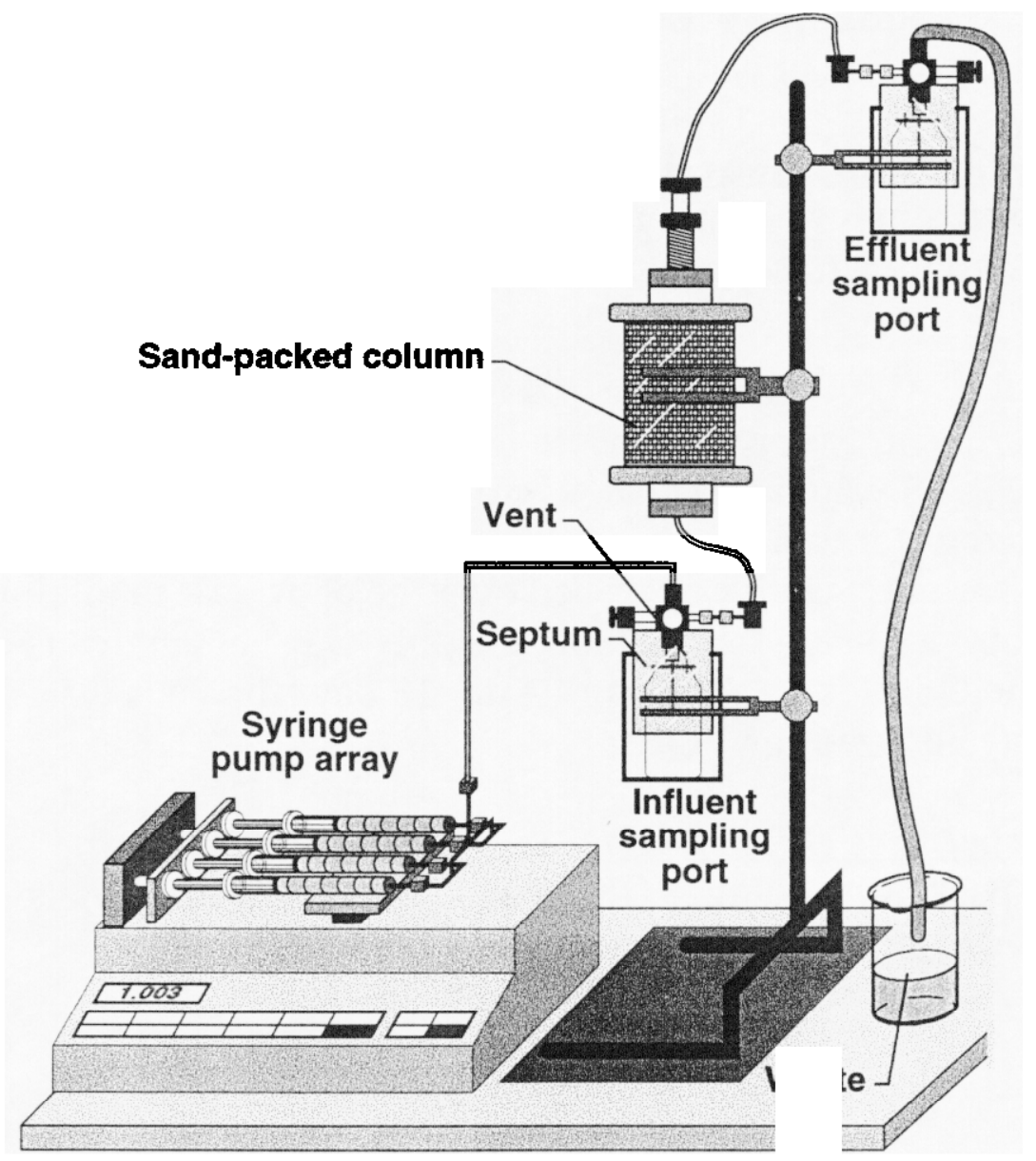




\section{Diffusion Experiment (Figure II)}

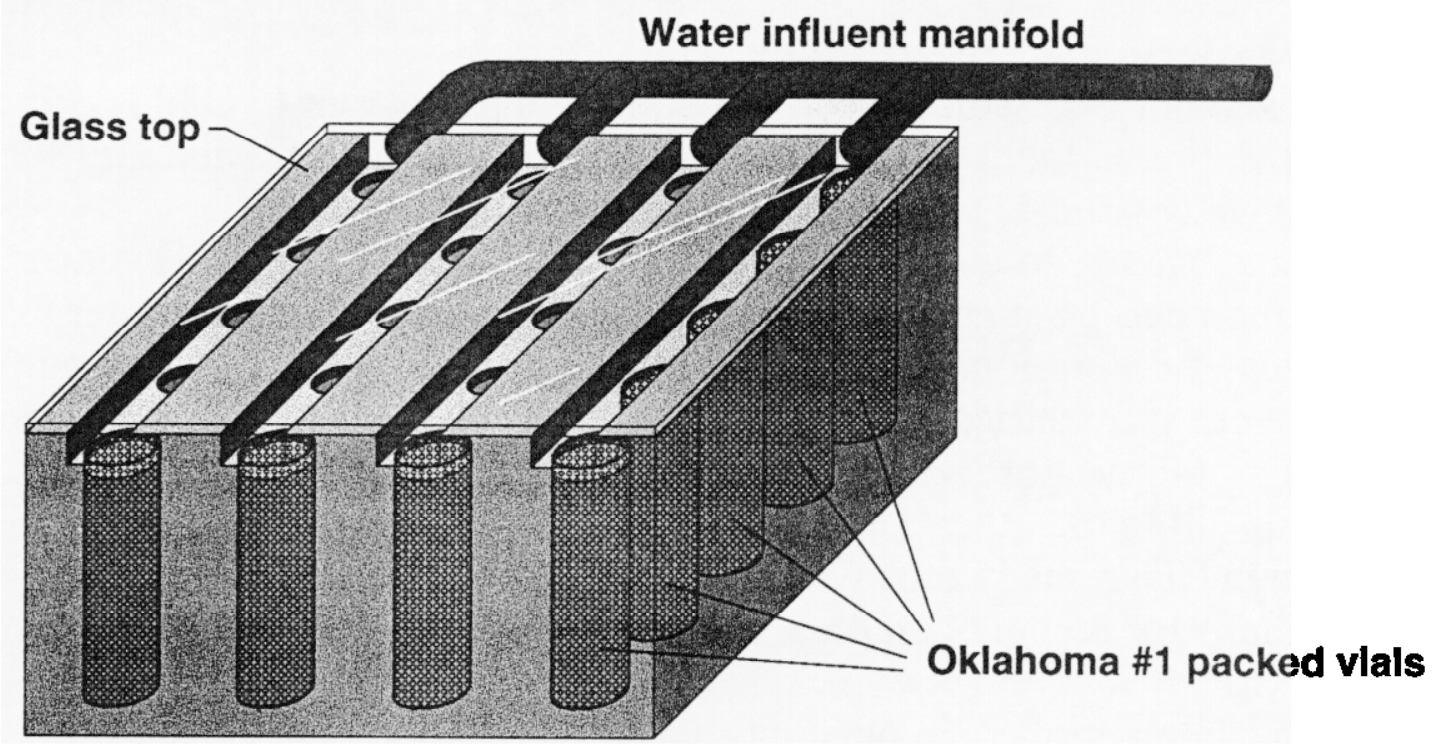

\section{Diffusion Vial Packing Assembly (Figure III)}

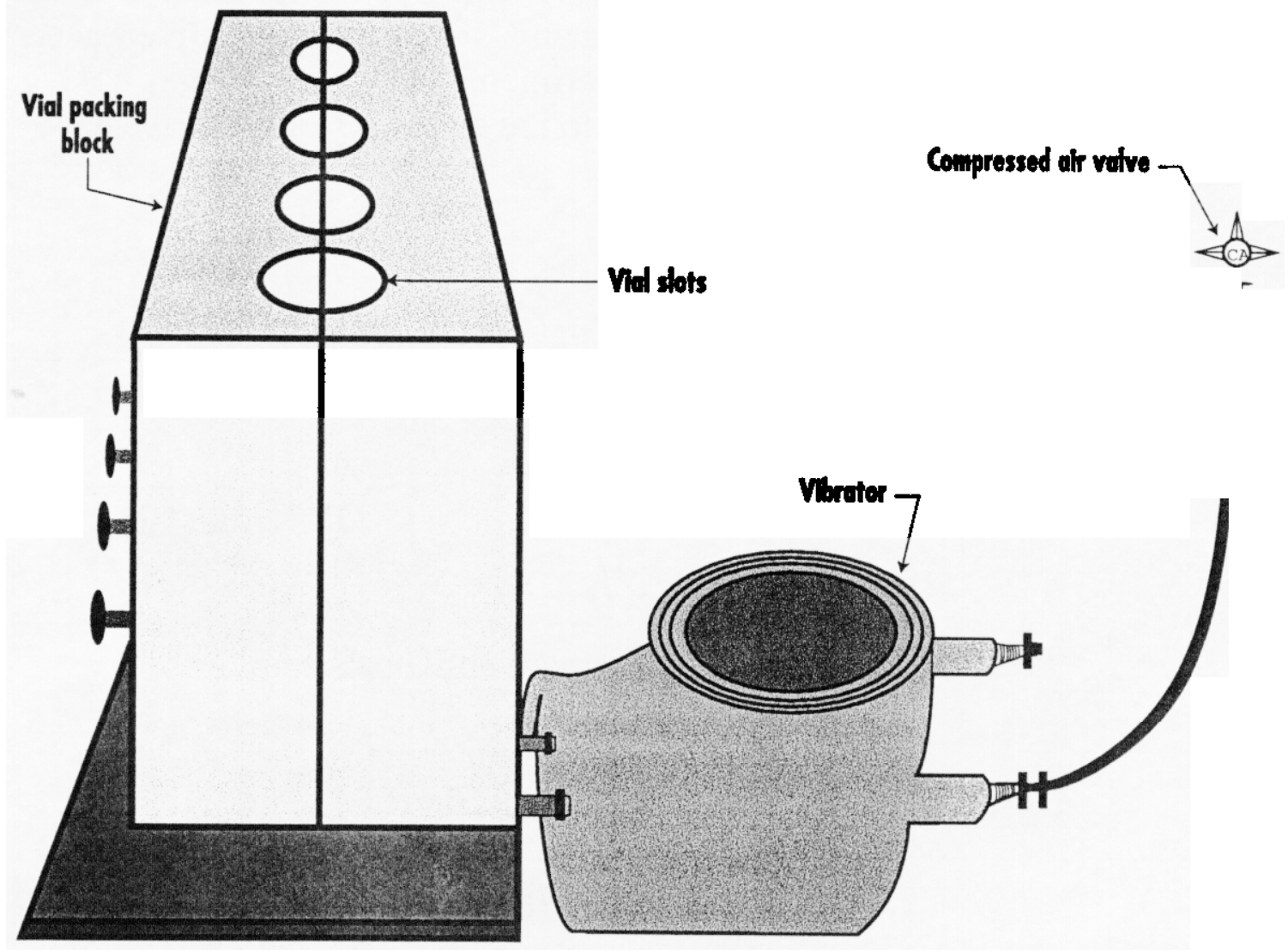




\section{Results}

Column Experiment

We analyzed our data with the computer modeling program CXTFIT, using the two-site kinetic model which takes into consideration diffusion into immobile water (Chart I). In comparing the $4 \mathrm{ml} / \mathrm{hr}$ and $8 \mathrm{ml} / \mathrm{hr}$ experiments, we observe no effect on retardation from velocity differences. From column curves, it appears that the kinetics of adsorption are faster than the kinetics of desorption. This explains why the cleanup and breakthrough curves are not symmetrical. From the $4 \mathrm{ml} / \mathrm{hr}$ cleanup curve we discovered that the long tail we observe is directly related to the kinetic effects. We do not see this tail in the other curves because we did not run the experiments long enough. To remedy this, we are currently running our Illite and NonIllite columns for 85 hours on breakthrough and $>240$ hours on cleanup.

As previously mentioned, in our first two column experiments when we began the cleanup phase with DI water we noticed a colloidal substance included in the effluent. After this was found to be illite clay, we researched and discovered that our $\mathrm{NaCl}$ tracer may be lowering the ionic strength of the solution and allowing for the illite clays to detach from the quartz grains during the cleanup phase. We have addressed this hypothesis in our current experiments by using Chloroform rather than $\mathrm{NaCl}$ as a conservative tracer. We discovered from our first column experiments that chloroform is not retarded. We are presently $>200$ hours into the cleanup phase and we have not seen any colloids.

\section{Diffusion Experiment}

Table $I$ is an example of the diffusion model used in the calculation of the tortuosity coefficient $(\Omega)$. This model utilizes the Excel Solver to derive $\Omega$ from the error function equation (Fetter,1992).

$$
\frac{C}{C o}=E R F C \frac{x}{2 \sqrt{D * t}}
$$

$$
D^{*}=D w(\Omega)
$$

where $\quad \mathrm{C}=$ Concentration at time $\mathrm{t}$ and location $\mathrm{x}$

$\mathrm{Co}=$ Initial concentration

$\mathrm{ERFC}=$ Complimentary error function

$D^{*}=$ Apparent diffusion coefficient

Dw $=$ Diffusion coefficient in water

$\Omega=$ Tortuosity coefficient 

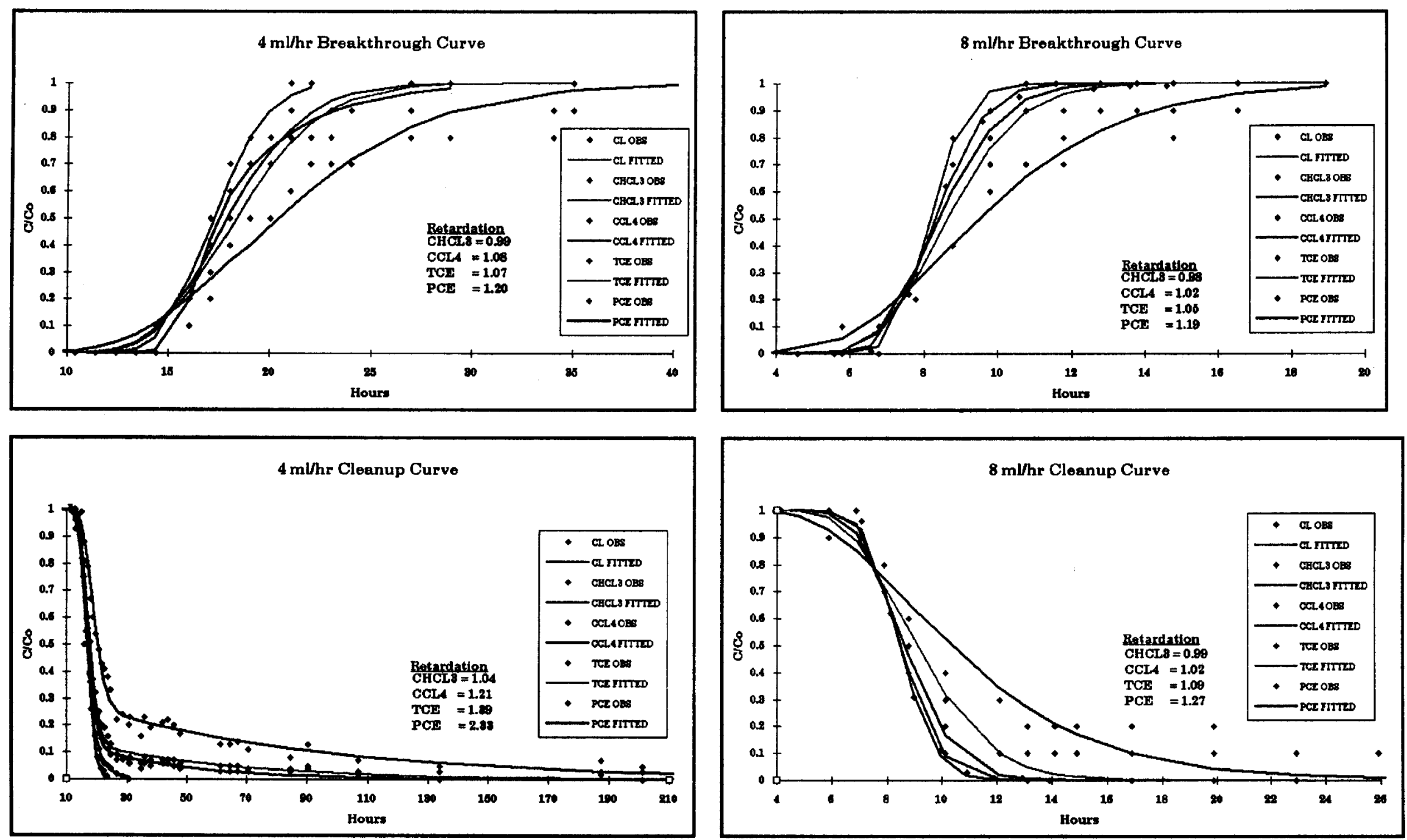

(Chart I) The fitted data was derived from a computer modeling program (CXTFIT, two site model). The obs (observed) data was collected by sampling the effluent from the column. The values for retardation were calculated using CXTFIT. 


\begin{tabular}{|c|c|c|c|c|}
\hline \multirow{2}{*}{\multicolumn{2}{|c|}{$\begin{array}{l}\text { Diffusion calculation: } \\
\mathrm{C} / \mathrm{Co}=\operatorname{erfc}\left(\mathrm{X} / 2\left(\mathrm{D}^{*} \mathrm{t}\right)^{\wedge} 0.5\right)\end{array}$}} & \multicolumn{3}{|c|}{ time, distance, and mass } \\
\hline & & \multirow[t]{2}{*}{$\mathbf{D}^{*}=\mathbf{D} w(\Omega)$} & \multirow{2}{*}{$\begin{array}{c}\text { Vial radius }(\mathrm{cm})= \\
\text { Vial area }\left(\mathrm{cm}^{\wedge} 2\right)=\end{array}$} & \multirow{2}{*}{$\begin{array}{r}0.657 \\
1.35\end{array}$} \\
\hline & & & \\
\hline$D^{*}=$ & 3.21E-06 & $\mathrm{cm}^{\wedge} 2 / \mathrm{sec}$ & Vial length $(\mathrm{cm})=$ & 4.318 \\
\hline $\mathrm{PCE} \mathrm{Dw}$ & $9.40 \mathrm{E}-06$ & $\mathrm{~cm}^{\wedge} 2 / \mathrm{sec}$ & & \\
\hline Tortuosity coeff. $(\Omega)$ & 0.341654356 & & & Time (sec) \\
\hline$t(\sec )=$ & $1,132,800$ & & $1 \mathrm{hr}=$ & 3,600 \\
\hline $\operatorname{Co}(\mathrm{ppb})=$ & 239.1 & & $1 \mathrm{day}=$ & 86,400 \\
\hline Porosity $=$ & 0.325 & & 1 week $=$ & 604,800 \\
\hline \multicolumn{2}{|c|}{ Total mass reported by the laboratory (ng) } & 226 & 1 month $=$ & $2,419,200$ \\
\hline Total mass (ng) & 226 & \multicolumn{2}{|c|}{ (Integrated over 2 vial lenghts) } & \\
\hline $\mathrm{x}(\mathrm{cm})$ & C (ug/) & $\mathrm{C}\left(\mathrm{ug} / \mathrm{cm}^{\wedge} 3\right)$ & Disk volume $\left(\mathrm{cm}^{\wedge} 3\right)$ & Mass (ug) \\
\hline 0.025 & 237.3323047 & 0.237332305 & 0.0675 & 0.016019931 \\
\hline 0.075 & 233.7975212 & 0.233797521 & 0.0675 & 0.015781333 \\
\hline 0.125 & 230.2645581 & 0.230264558 & 0.0675 & 0.015542858 \\
\hline 0.175 & 226.7346269 & 0.226734627 & 0.0675 & 0.015304587 \\
\hline 0.225 & 223.2089362 & 0.223208936 & 0.0675 & 0.015066603 \\
\hline 0.275 & 219.68869 & 0.21968869 & 0.0675 & 0.014828987 \\
\hline 0.325 & 216.1750868 & 0.216175087 & 0.0675 & 0.014591818 \\
\hline 0.375 & 212.6693181 & 0.212669318 & 0.0675 & 0.014355179 \\
\hline 0.425 & 209.1725677 & 0.209172568 & 0.0675 & 0.014119148 \\
\hline 0.475 & 205.6860099 & 0.20568601 & 0.0675 & 0.013883806 \\
\hline 0.525 & 202.2108086 & 0.202210809 & 0.0675 & 0.01364923 \\
\hline & & & $\ldots \ldots \ldots$ & \\
\hline & & & & \\
\hline
\end{tabular}

(Table I) The diffusion model utilizes the Excel Solver to derive tortuosity $(\Omega)$ from the error function equation. 
We discovered from the column experiment that chloroform is not retarded. This discovery allows for a new method of calculating retardation (R) by dividing the $\Omega$ of chloroform by the $\Omega$ of the VOC of interest.

$$
R=\Omega C_{H C L} / \Omega V O C
$$

The retardation values from the diffusion experiment coincide very well with those from the column experiments. This tells us that the diffusion apparatus is working and we can begin testing different grain sized materials.

\section{Summary}

These experiments have made significant headway into the problem of understanding retardation. From the column experiments we have discovered: differences in velocity has very little effect on retardation, however it does limit our ability to observe kinetic effects, our conservative tracer $\mathrm{NaCl}$ is disrupting the ionic strength of the solution and allowing for the illite clay to detach from the quartz grains, chloroform is not retarded and we can now use it as a tracer, and the kinetics of adsorption are faster than the kinetics of desorption. From the diffusion experiment we discovered a new method for measuring retardation, and that our retardation values from the diffusion model coincide with the CXTFIT values from the column experiments. These experiments have provided very promising results, however a great deal of research still remains before we can fully understand retardation.

\section{Continued Research}

Further research is currently underway to determine why the kinetics of adsorption occur faster than those for desorption. We are presently conducting column experiments to determine the effect of illite clay and $\mathrm{NaCl}$ on retardation. Future column and diffusion experiments will focus on measuring diffusion and retardation into different aquifer materials. 


\begin{tabular}{|c|c|c|c|c|c|}
\hline \multicolumn{6}{|c|}{ Retardation Comparisons on Oklahoma \#1 Sand } \\
\hline \multirow[t]{2}{*}{ Chemical } & $4 \mathrm{ml} / \mathrm{hr} \mathrm{BT}$ & $4 \mathrm{ml} / \mathrm{hr} \mathrm{CL}$ & $8 \mathrm{mlhr}$ BT & $8 \mathrm{ml} / \mathrm{hr} \mathrm{CL}$ & Diffusion \\
\hline & CXIFIT R & \begin{tabular}{|l|} 
CXIFIT R \\
\end{tabular} & CXIFIT R & CXIFIT R & $\mathbf{R}$ \\
\hline CHCl3 & 0.99 & 1.04 & 0.98 & 0.99 & 1.00 \\
\hline \multirow{2}{*}{$\overline{\mathrm{CCl} 4}$} & 108 & 101 & 102 & 102 & 138 \\
\hline & & & & & \\
\hline TCE & 1.07 & 1.39 & 1.05 & 1.07 & 1.04 \\
\hline \multirow[t]{2}{*}{ PCE } & 1.20 & 2.33 & 1.19 & 1.27 & 1.11 \\
\hline & & & & & \\
\hline \multirow{2}{*}{\multicolumn{3}{|c|}{$\begin{array}{l}\mathrm{BT}=\text { Breakthrough Experiment } \\
\mathrm{CL}=\text { Cleanup Experiment }\end{array}$}} & \multicolumn{3}{|c|}{ Column bulk density $=1.8$} \\
\hline & & & Column por & sity $=0.32$ & \\
\hline \multirow{2}{*}{\multicolumn{3}{|c|}{ Diffusion bulk density $=1.777$}} & & & \\
\hline \multicolumn{2}{|c|}{ Diffusion porosity $=0.325$} & & & & \\
\hline & & & & & \\
\hline
\end{tabular}

(Table II) Comparison between the CXTFIT values for retardation and the diffusion model's values for retardation. Again note the larger $R$ values for the $4 \mathrm{ml} / \mathrm{hr}$ cleanup in which kinetic effects are observed.

\section{References}

Piwoni, Marvin D. and Banerjee, Pinaki. 1989. Sorption of volatile organic solvents from aqueous solution onto subsurface solids. Journal of Contaminant Hydrology, 4:163-179

Fetter, C. W. 1992. Contaminant Hydrogeology, (Macmillan College Publishing Company, 1993) 458p. 


\title{
Evaluation of a New Model for the Prediction of Dermal Absorption
}

\author{
Susanne Meissner
}
California Polytechnic State University San Luis Obispo

\section{Lawrence Livermore National Laboratory Livermore, California 94550}

$$
\text { May 8, } 1996
$$

Prepared in the fulfillment of the requirements of the Science and Engineering Research Semester under the direction of Garrett Keating, Research Mentor, in the Lawrence Livermore National Laboratory.

* This research was supported in part by an appointment to the U.S. Department of Energy Science and Engineering Research Semester (hereinafter called SERS) program administered by LLNL under Contract W-7405-Eng- 48 with Lawrence Livermore National Laboratory.

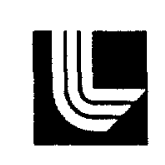




\section{Abstract}

The primary goal of this research project is to determine the uptake rate of chemicals into the skin from three environmental media, water, soil and indoor dust. The U.S. Environmental Protection Agency (EPA) has proposed a dermal uptake model that predicts higher uptake for certain chemicals than previously estimated. Our study focuses on short-term, low level exposure to water contaminants that are comparable to actual environmental exposures. In previous studies such an approach was not achievable due to the lack of sensitive equipment required for measurements on this low of a scale. With the use of Accelerator Mass Spectrometry we have the sensitivity to detect minute amounts of radiolabeled chemical in skin samples following exposures to chemicals at parts per billion $(\mu \mathrm{g} / \mathrm{L})$ concentrations. Using time points from 1 minute to $60 \mathrm{~min}$, we will produce uptake curves from which kinetic parameters can be estimated. These results will be incorporated into the existing EPA model so a more realistic approach to setting contamination standards can be reached. 


\section{Introduction}

Dermal absorption studies traditionally use a dual chambered diffusion cell in which the skin acts as a membrane through which chemical penetration is measured. The assessment of chemical diffusion consists of a series of chemical concentration measurements of the receptor fluid. The samples are analyzed with liquid scintillation counting to detect the disintegrations per minute from the radiolabled chemicals which diffuse through the skin. Due to the limit of detection of scintillation counting, the dosing concentration must be high. A permeability coefficient $\left(\mathrm{K}_{\mathrm{p} \mathrm{g} \mathrm{cm} / \mathrm{hr}}\right)$ is obtained after a constant rate of diffusion is detected. The $K_{p}$ is used to predict the amount of chemical absorbed into the skin over a given period of time. It typically takes hours to reach a steady state of diffusion. The long exposure time and elevated dosing concentrations do not represent the type of exposure relevant to current environmental hazards.

A proposed EPA model treats the skin as a two layer membrane. The stratum corneum and epidermis comprise two distinct layers above the vasculature of the dermis. This new model takes into account the $\mathrm{Kp}$ and physicochemical properties to predict the chemical's uptake from water (Cleek and Bunge, 1992; EPA, 1992; Shaum et al., 1993). Due to the variation in physical properties of the 2 skin layers, the rate at which chemicals diffuse through each layer will vary. Steady state uptake represents a combination of these two rates. The old model measures the combined rate which is relevant once the diffusion of a chemical reaches a steady state diffusion. There is a lag time from the time diffusion starts and a steady rate of diffusion is maintained. During this time the skin becomes saturated with chemical and acts as reservoir. The new model characterizes the short term exposure risks. This short-term uptake rate is more relevant to estimate dermal absorption from showering and bathing. 
Using Accelerator Mass Spectrometry (AMS) we were able to directly measure the small amount of chemical absorbed into the skin over a short period of time. The methods used in this research permit direct measurement of uptake from skin as a result of short term exposure to low concentrations of contaminant in a dosing medium. Our study will compare these measurements with the estimates of the proposed model.

\section{Materials and Methods}

Test Chemical: The test chemical used was $\left[1,2-{ }^{14} \mathrm{C}\right]$ trichloroethylene (TCE, 5.4 $\mathrm{mCi} / \mathrm{mmol}, \geq 98 \%$ purity, Sigma Chemical Co., St. Louis, MO). The radiolabled TCE was shipped in a sealed ampule which was opened into $5 \mathrm{ml}$ of methanol held in ice to yield a continuously refrigerated stock of labeled solution $(0.039 \mu \mathrm{Ci} / \mu \mathrm{l})$.

Tissue: Full-thickness breast skin was obtained from mammoplasties performed at the University of California San Francisco Medical School. The tissue was transported on ice, refrigerated, and used within $48 \mathrm{hr}$ of being removed. The tissue was rinsed with distilled water and excess fat was removed before each experiment.

Dermal Exposure Experiments: Tissue was exposed to a dilute aqueous solution of TCE $(5.0 \pm 0.85 \mu \mathrm{g} / \mathrm{L})$ at a solution temperature of $22^{\circ} \mathrm{C} \pm 2$. The dosing solution was prepared by adding $3 \mu \mathrm{l}$ of TCE stock solution to $250 \mathrm{ml}$ of water. Tissue discs were obtained from the bulk specimen by marking an area of $3.14 \mathrm{~cm}^{2}$ with a 2$\mathrm{cm}$ diameter cork bore and cutting out the area with surgical scissors. Tissue discs were placed between the two chambers of the Low Flow Diffusion Cell (LFDC, RCR Inc., Navato CA, Fig. 1) The LFDC cap was lined with foil and the threads were wrapped with Teflon tape to minimize loss of TCE; an absorbent pad and aluminum foil were placed between the base of the LFDC and tissue sample to create a tighter seal once the screw clap was secured. A spin bar $(0.8 \mathrm{~cm}$ long) was placed in the receptor 
compartment and donor solution $(3.8 \mathrm{ml})$ was pipetted into the donor chamber and the cap tightly screwed in place. The LFDC was set on a stir plate and the spin bar was elevated by placing a magnet on the exterior of the receptor compartment. The stir plate was then turned on, causing the spin bar to toggle freely. The skin was exposed for $1,5,15,30$, and $60 \mathrm{~min}$. The dosing solution concentration was monitored over the course of the experiments by transferring three $500 \mu \mathrm{l}$ samples using an automatic pipetter to liquid scintillation vials containing $15 \mathrm{ml}$ of Universol (ICN Biomedical, Inc., Irvine, $\mathrm{CA}$ ) for quantification of ${ }^{14} \mathrm{C}$ in a Tri-Carb 4530 scintillation counter (Packard Instrument Co.) Controls were exposed to distilled water.

Tissue Sampling: Samples were processed quickly because of the volatility of TCE. The skin was removed from of the cell, blotted dry and spread out on a aluminum-lined Teflon board. Three to five skin cores were taken from the tissue disc by hammering a stainless steel tube $\left(0.054 \mathrm{~cm}^{2}\right)$ through the exposed tissue. Each skin core was transferred to a quartz tube that was flash frozen in liquid nitrogen, placed on dry ice, and stored in a $-70^{\circ} \mathrm{C}$ freezer. The tissue cores had an average weight of $4.3 \pm 1.3 \mathrm{mg}$. Control tissue was handled in the same manner.

Tissue Analysis: Tissue cores were analyzed for ${ }^{14} \mathrm{C}$ content by Accelerator Mass Spectrometry (AMS), an isotope-ratio mass spectrometric method of quantifying radioisotopes independent of their decay times (Vogel and Turtletaub, 1991). AMS can detect femtomoles of ${ }^{14} \mathrm{C}\left(10^{-15}\right)$ atoms in a sample. AMS requires that for ${ }^{14} \mathrm{C}$ analysis, samples need to be converted to graphite prior to introduction into the accelerator. To do so, tubes containing the tissue samples were flame sealed and the sample was combusted in to $\mathrm{CO}_{2}$ gas. The $\mathrm{CO}_{2}$ gas was transferred under vacuum to a second quartz tube and re-solidified into graphite powder (Vogel, 1992). Carbon atoms are stripped from the graphite by ion bombardment and introduced into a particle accelerator and a ratio of ${ }^{14} \mathrm{C}$ atoms to ${ }^{13} \mathrm{C}$ atoms was determined. Total carbon in the skin samples was determined by measuring the carbon content of the skin $(\% \mathrm{C})$ with a Carlo Erba NA-1500 CNHS Analyzer (Fisons Instruments, Milan, Italy). Sample 
weights were then multiplied by this percentage. The ratio was then converted to femtomoles of ${ }^{14} \mathrm{C}-\mathrm{TCE}$ in the tissue with the following equation:

Total femtoM ${ }^{14} \mathrm{C}$ in samples =

FM $\times \frac{5.9 \times 10^{10} C^{14} \text { atoms } / g \text { carbon }}{6.02 \times 10^{23} C^{14} \text { atoms } / \text { mole } C^{14}} \times\langle$ sample wt $(g) \times \% C$ in skin $\rangle \times \frac{10^{15} \text { femtograms }}{\text { gram }}$

Statistical Analyses: The concentrations of the exposure solution used in there experiments were not exactly the same. The variation was accounted for before the comparison of data by normalizing the data to $5 \mu \mathrm{g} / \mathrm{L}$. Statistical comparisons ( $t$ tests, $p$ - values $\leq 0.05$ ) of chemical uptake were performed on the normalized data.

\section{$\underline{\text { Results and Conclusion }}$}

Carbon content cores taken from non-exposed areas of the breast tissue reviled a carbon content of $22 \%$. This was multiplied by the average weight of a tissue core $(4.3$ $\pm 1.3 \mathrm{mg}$ ) to get the weight of carbon in a sample. This was used to calculate the amount to ${ }^{14} \mathrm{C}$ in a sample. Each data set from AMS was converted to femtoM of ${ }^{14} \mathrm{C}$ TCE for the specific time points. Data from the three subjects were compared (Fig. 2). Between 0 and $5 \mathrm{~min}$ there was no significant difference in TCE uptake for the three samples. At $15 \mathrm{~min}$, subjects 2 and 3 did not show significant difference between their level of uptake. However both subjects 2 and 3 had significantly lower uptake ( $p \leq$ 0.005 ) than subject 1 . At $30 \mathrm{~min}$ all three subjects have uptake rates that are significantly different from one another $(p \leq 0.005)$. Subject 2 absorbed less TCE than subject 3 . Subject 1 absorbed the most TCE during $30 \mathrm{~min}$. At $60 \mathrm{~min}$ subjects 1 and 2 did not statistically differ from one another and their TCE absorption was significantly higher $(p \leq 0.005)$ than subject 3 . The variation between these subjects may reflect the inherent variation between the physiology of skin tissue. 
The absorption data sets from the three subjects were averaged and compared to skin absorption predicted by the traditional EPA model and the New model (Fig. 3) Experimental values were converted to $\mathrm{ng} / \mathrm{cm}^{2}$. On the whole the experimental data correlated with the old EPA predictions. Even through the experimental data fits the old EPA predictions best, that model predicts uptake as a linear increase of absorbed contaminant over time, where as the experimental data shows a leveling off of absorbed TCE over $60 \mathrm{~min}$. This leveling off indicates that the tissue is reaching its point of saturation. The new model over predicts the amount of absorption by an average factor of 6 and does not seem to accurately predict chemical absorption. 


\section{References}

Cleek, R.L., and Bunge, A.L. (1993). "A new method for estimating dermal absorption from chemical exposure. 1. General approach." Pharmaceutical Res. 10:497-506.

Schaum, J., K. Hoang, R. Kinerson, J. Moya, and R. Wang. 1994. Estimating dermal and inhalation exposure to volatile chemicals in domestic water. In: R.G.M. Wang, ed. Drinking Water Contamination and Health: Integration of Exposure Assessment, Toxicology and Risk Assessment. Marcek Dekker, Inc., New York, pp. 305-321.

US Environmental Protection Agency (EPA, 1992). Dermal exposure assessment: principles and applications. Report No. EPA/600/8-91/011B. Office of Health and Environmental Assessment, U.s. Environmental Protection Agency, Washington, DC.

Vogel, JS, and KW Turtletaub (1991) Biomolecular tracing through accelerator mass spectrometry. Trac-Trends Anal Chem 11, 142-149.

Vogel, JS (1992) Rapid production of graphite without contamination of biomedical AMS. Radiocarbon 34, 344-350. 


\section{(Fig. 1) Low Flow Diffusion Cell (LFDC)}

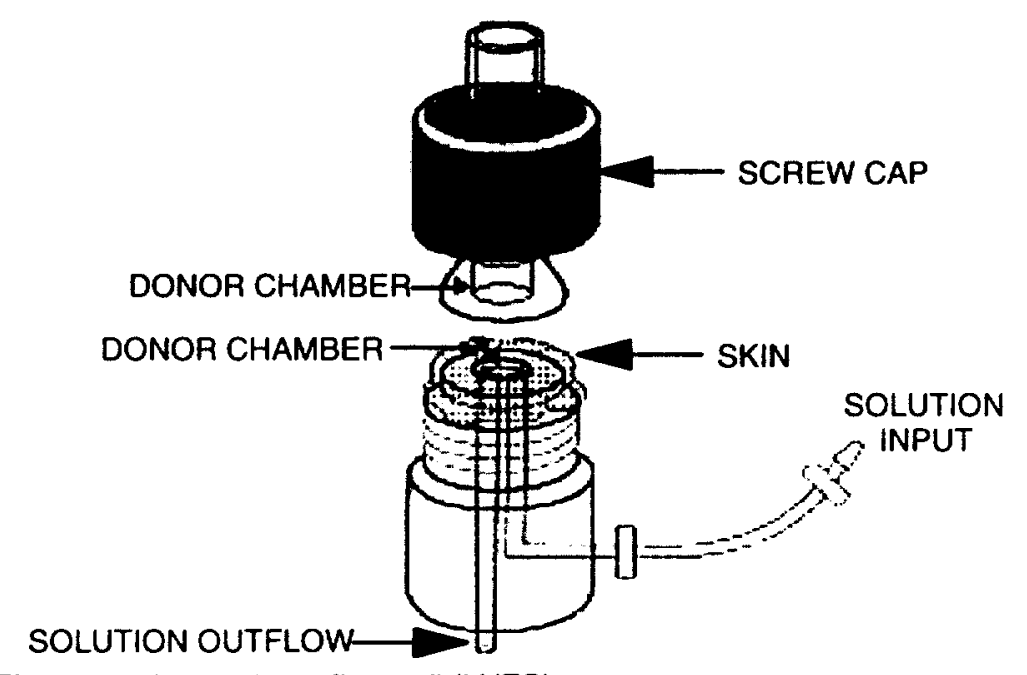

Figure 1 - Low-volume flow cell (LVFC). 


\section{(Fig. 2) Short-term, dermal uptake of TCE}

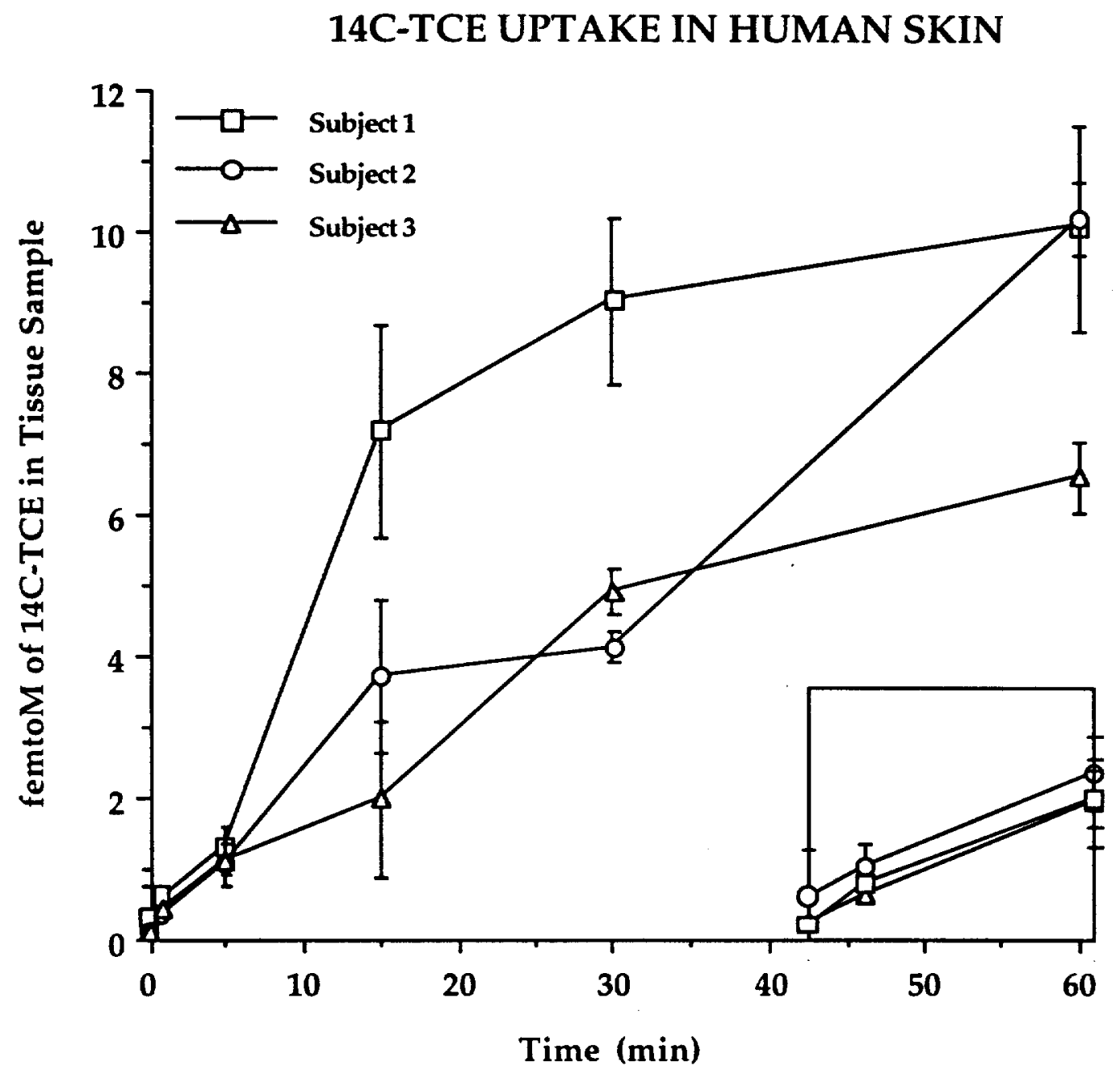




\section{(Fig. 3) Comparison of Measurements and Models}

\begin{tabular}{|c|c|c|c|}
\hline \multirow[t]{2}{*}{$\begin{array}{l}\text { Time } \\
\text { (min) }\end{array}$} & \multirow{2}{*}{$\begin{array}{c}\text { Measured Uptake } \\
\left(\mathrm{ng} / \mathrm{cm}^{2}\right) \\
(\mathrm{n}=3)\end{array}$} & \multicolumn{2}{|c|}{$\begin{array}{c}\text { Skin Absorption } \\
\text { Model }\end{array}$} \\
\hline & & New Model & EPA Model* \\
\hline 1 & $0.0022 \pm 0.0009$ & 0.0211 & 0.001333 \\
\hline 5 & $0.0052 \pm 0.0009$ & 0.0471 & 0.006667 \\
\hline 15 & $0.0187 \pm 0.0122$ & 0.0816 & 0.02 \\
\hline 30 & $0.0260 \pm 0.0123$ & 0.1154 & 0.04 \\
\hline 60 & $0.0384 \pm 0.00957$ & 0.1631 & 0.08 \\
\hline
\end{tabular}

${ }^{*}$ EPA model predictions based on TCE $K_{p}$ value of $0.016 \mathrm{~cm} / \mathrm{hr}$. 



\title{
Conversion of Character-Based Interface to Graphical Interface for ESIS *
}

\author{
Tri M. Nguyen \\ Loyola Marymount University \\ Computations \& Communications Engineering Group \\ Computer Applications, Sciences, \& Engineering Division \\ Lawrence Livermore National Laboratory \\ Livermore, California 94550
}

April 25, 1996

Prepare in partial fulfillment of the requirements of the Science and Engineering Research Semester under the direction of John Wade, mentor, in the Lawrence Livermore National Laboratory.

* This research was supported in part by an appointment to the U.S. Department of Energy's Science and Engineering Research Semester (hereinafter called SERS) program administered by LLNL under Contract W-7405-Eng-48 with Lawrence Livermore

National Laboratory.

If this paper is to be published, a copyright disclaimer must also appear on the cover sheet as follows:

By acceptance of this article, the publisher or recipient acknowledges the U.S. Government's right to retain a non-exclusive, royalty-free license in and to any copyright covering 
Apple and Macintosh are trademarks of Apple Computer, Inc. DEC and VAX are trademarks of Digital Equipment Corporation. Oracle, Oracle 7, Oracle Forms 3.0, Forms 3.0, Oracle Forms 4.5, and Forms 4.5 are trademarks of the Oracle Corporation. OSF, OSF/1, OSF/Motif, and Motif are trademarks of the Open Software Foundation, Inc. 


\title{
Conversion of Character-Based Interface to Graphical Interface for ESIS
}

\author{
Tri M. Nguyen \\ Loyola Marymount University \\ Computations \& Communications Engineering Group \\ Computer Applications Sciences \& Engineering Department \\ Lawrence Livermore National Laboratory
}

\begin{abstract}
ESIS is an information system for tracking manpower, work orders, and cost accounts for the Electronic Services and Electronic Manufacturing departments. My responsibilities for ESIS are: to fix any errors and crashes, to manage the configurations, to provide documentation about the system, to convert the character-based interface to a graphical interface, and to merge two databases. In particular, this paper will focus on the conversion of a character-based interface to a graphical interface. At the present time, only two modules of ESIS have been converted. After the current bugs and errors in other parts of ESIS have fixed, then the conversion process will continue.
\end{abstract}




\section{Table of Contents}

Abstract

1. Introduction 1

1.1 Overview 1

1.2 Problem Definition 1

1.3 Requirements 3

2. Background 4

2.1 System Components 4

2.2 Business Procedures 5

3. Conversion Process 7

3.1 Before Conversion 7

3.2 Work Involved in Conversion 10

4. Results \& Discussion 16

4.1 Accomplishments 16

4.2 Benefits \& Disadvantages 16

$\begin{array}{ll}4.3 \text { Next Steps } & 17\end{array}$ 


\section{Introduction}

\subsection{Overview}

For small companies or departments, keeping track of employees and work orders is relative easy. In these situations, paper forms and punch cards could be appropriate. However, as companies and departments grow in size -- in terms of personnel and orders -- the method of tracking just mentioned becomes daunting and time-consuming. This scenario applies to the Electronic Services and Electronic Manufacturing Departments at Lawrence Livermore National Laboratory. The tracking system of these two departments has already moved from paper forms to ESIS, an information system. However, there are some problems with the current system. The project described in this paper addresses these problems and upgrades to the current electronic forms. In particular, this paper will discuss the problems, background, methods (used to resolve the problems) and results of the project. The problems and requirements will be defined in the next two sub-sections

\subsection{Problem Definition}

The current interface to ESIS is a set of character-based (CB) forms. To access ESIS, a user would 'telnet' into a server machine. The user would be presented with screens as in Figures 1. In order to perform the tasks and transactions, the user must use the keyboard, esp. tabs, to navigate between forms and between text displays and to enter values into fields. 


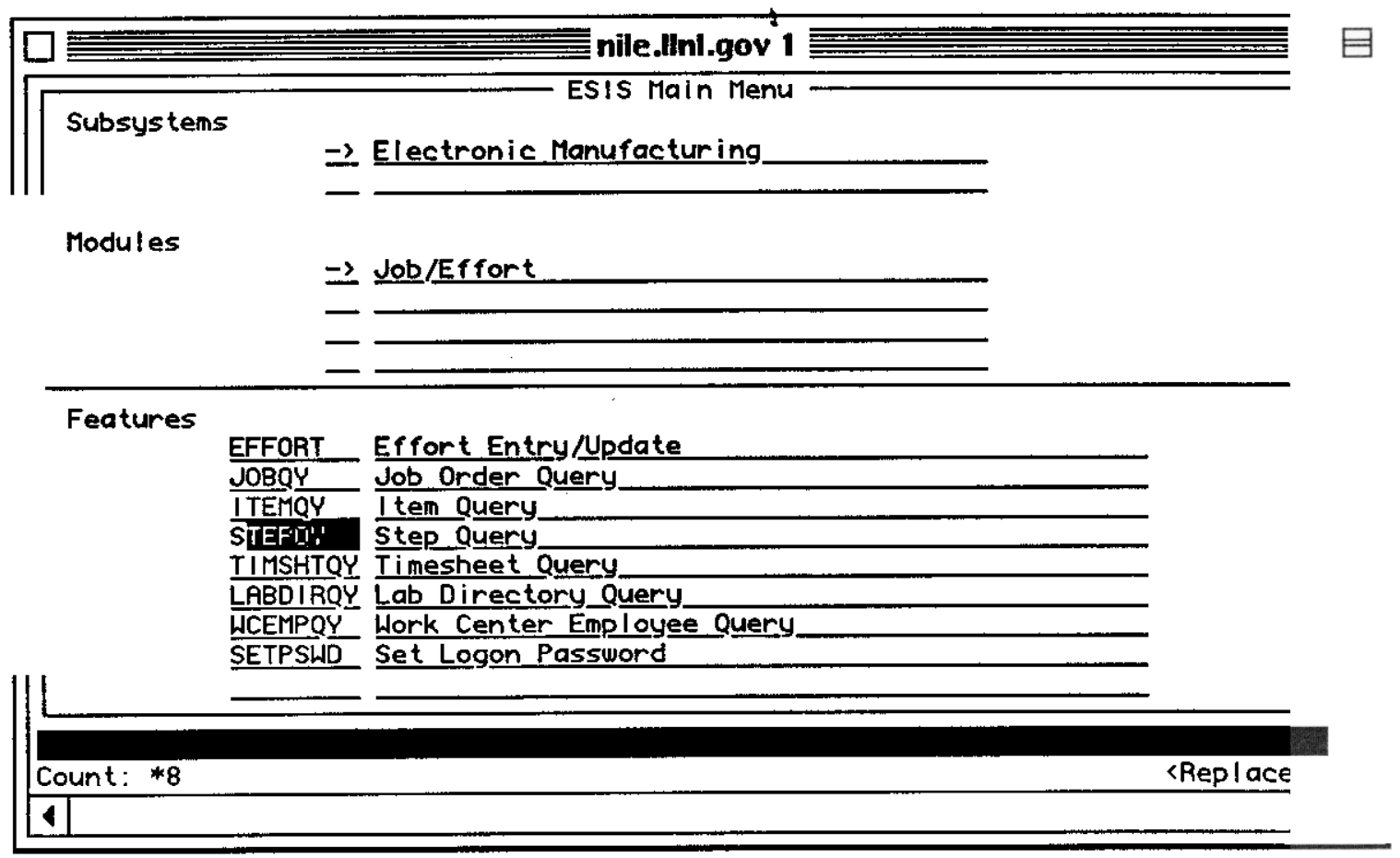

Figure 1: Menu form with character-based interface 


\title{
Testing Candidate Alloys for Resistance to Microbial Induced Corrosion
}

\author{
Angel Rivera \\ Universidad del Turabo \\ Lawrence Livermore National Laboratory \\ Livermore, California 94550
}

May 8, 1996

This paper was prepare in partial fulfillment of the Science and Engineering Research Semester under the direction of Dr. Joanne Horn Research Mentor, in the Lawrence Livermore National Laboratory.

This research was supported in part by an appointment to the U.S. Department of Energy Science and Engineering Research Semester (SERS) program administered by LLNL under Contract W-7405-ENG-48 with Lawrence Livermore National Laboratory . 


\section{Table of Contents}

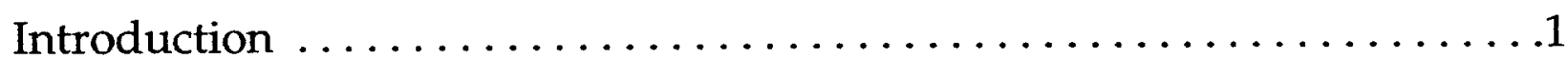

Acid Producttion Test $\ldots \ldots \ldots \ldots \ldots \ldots \ldots \ldots \ldots \ldots \ldots$

Hydrogen sulfide Production test $\ldots \ldots \ldots \ldots \ldots \ldots \ldots \ldots \ldots$

Generation of exopolisaccharides $\ldots \ldots \ldots \ldots \ldots \ldots \ldots \ldots \ldots$

Conclusion/Discussion $\ldots \ldots \ldots \ldots \ldots \ldots \ldots \ldots \ldots \ldots \ldots \ldots \ldots \ldots \ldots \ldots \ldots \ldots$

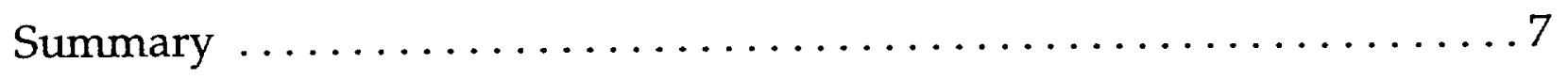

Figures $\ldots \ldots \ldots \ldots \ldots \ldots \ldots \ldots \ldots \ldots \ldots \ldots \ldots \ldots \ldots \ldots$

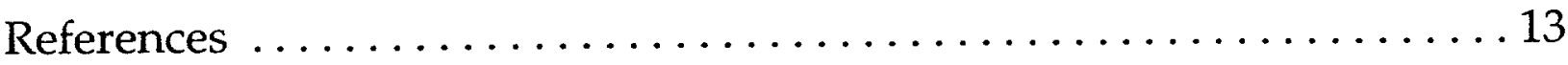




\begin{abstract}
Angel Rivera

Earth Science Department, Yucca Mountain Project
\end{abstract}

Testing Candidate Alloys for Resistance to Microbial Induced Corrosion

The growth of native and introduced bacteria in the Yucca Mountain repository site may jeopardize the integrity of geologic nuclear waste disposal facilities by directly promoting the corrosion of repository components. Therefore demands a characterization of microbial effects on the repository materials. We investigate the presence of bacteria in samples retrieved from the repository site. These were screened for microbial activities associated with Microbial Induced Corrosion (MIC):

- Acid production

- Sulfate reduction and production of hydrogen sulfide

- Generation of exopolysaccharides (biofilm "slime")

Acid production was found to be highly dependent on the presence of glucose in the midea. Howeverglucose-affected alterations of $\mathrm{pH}$ may be reduced by the presence of peptone. As a result of these activities quantification in terms of rate, extent, and correlation to associated growth rates, are being performed. These studies indicate the conditions requisite for metal corrosion by native repository bacteria. They also indicate ideal conditions for accelerated testing of candidate alloys for their resistance to MIC. 


\section{INTRODUCTION}

Bacteria and other microorganisms are the most ubiquitous life forms on earth. Bacteria resistant to long term, high-level radiation were found growing in the damage core of the failed nuclear reactor at Three Mile Island. Many types of aerobic and anaerobic microorganisms have been associated with and isolated from nuclear reactor systems at commercial power plants and from defense spent fuel storage pools. Thus, long term exposure to high-level radiation in nutrient-depleted environments does not appear to inhibit microbial growth and activity.

For licensing a high-level nuclear waste (HLW) repository to be constructed by the US Department of Energy (DOE), the Nuclear Regulatory Commission (NRC) regulation 10 CFR 60.113 requires waste packages to provide substantially complete containment of radionuclides for a minimum period of 300 to 1,000 years. The challenge then is to identify and prioritized the significant processes and events that are likely to affect the waste package performance during the radioactive decay period.

The Department of Energy is engaged in a suitability study for a potential geological repository at Yucca Mountain, Nevada, for the containment and storage of commercially generated spent fuel and defense high-level nuclear waste. There is growing recognition of the role that biotic factors can play in this repository, either directly through 
microbially induced corrosion (MIC), or indirectly by altering the chemical environment or contributing to the transport of radionuclides. This may also be applied to any waste deposition scenario, including temporary above-ground waste impoundment.

The aim of this research is to identify microbes and their associated metabolic paths that operate or are enhanced by the modified environmental conditions expected after subsurface waste emplacement and evaluate the potential biological processes that may compromise the integrity of the waste packages. 


\section{Methods}

\section{Acid Production by YM Isolates (pH Curves)}

Media -Dependent Alteration of $\mathrm{pH}$ by Specific Yucca Mountain Isolates

Yucca Mountain bacteria identified in initial visual screening (growth in $\mathrm{R} 2$ media $+0.5 \%$ glucose, together with $\mathrm{pH}$ indicators) as acid-producers, were grown in pre cultures and inoculated (1:100) into $100 \mathrm{ml}$ growth media (R2, R2 $+0.5 \%$ glucose, $\mathrm{R} 2+0.75 \%$ peptone, or $\mathrm{R} 2+0.5 \%$ glucose $+0.75 \%$ peptone, prepared using standard protocols under both aerobic and anaerobic conditions).

Inoculated aerobic cultures were incubated by shaking (200 rpm) at either 30 or 50 degrees $C$., while anaerobic cultures, contained in sealed serum bottles were incubated without shaking. $10 \mathrm{ml}$ of media (together with cells) were removed periodically from incubating cultures and the $\mathrm{pH}$ was determined using a $\mathrm{pH}$ meter.

\section{Hydrogen Sulfide Production by YM Isolates}

Yucca Mountain bacteria identified in initial visual screening (growth media composed of R2 agar (Difco), a low nutrient formulation, supplemented with $0.75 \%$ proteose peptone \#3 (Difco), and $0.05 \%$ lead acetate) were grown in pre cultures and inoculated into $100 \mathrm{ml}$ growth media $\mathrm{R} 2+0.75 \%$ peptone, using standard protocols under both aerobic and anaerobic conditions. 
Inoculated bacteria were incubated at either $30^{\circ}$ or $50^{\circ} \mathrm{C}$ and removed periodically from incubator for sulfide testing. Sulfide testing was perform running a standard curve in every test, with specifics concentration of sodium sulfite to be use as reference to determine the concentration of hydrogen sulfide produce by the isolates.

\section{Exopolisaccharide (biofilm "slime") Production}

Production of viscous polymeric sugar exported by cells (exopolisaccharides) is evidenced by glistening gelatinous material surrounding bacterial colonies. Isolates were streaked on R2 agar (Difco), a low nutrient formulation, and incubated for 3 days at either room temperature or $50^{\circ} \mathrm{C}$. 


\section{CONCLUSION}

Bacterial strains were isolated from Yucca Mountain (Nevada), a potential nuclear waste repository site. These were characterized to determine their potential for growth and metal corrosion, under a variety of conditions. Whole communities isolated from Yucca Mountain geologic samples demonstrated significant growth even in low nutrient conditions and at temperatures up to 50 degrees $C$, reaching cell densities of (greater than?) $10^{8}$ cells $/ \mathrm{ml}$ (Fig. 1). Given Yucca Mountain bacterial isolates, most probably spore-forming bacteria, were capable of surviving repeated exposure to temperatures as high as 120 degrees $C$. Thus, it may be anticipated that with minimal nutrients, at least a subset of the microbial community present at the repository site, could survive and grow under the elevated temperatures expected after nuclear waste emplacement.

Visual screening of 60 Yucca Mountain bacterial isolates were initially employed to determine which ones possessed activities associated with microbially induced corrosion. The screening program demonstrated that specific isolates were capable of producing acid (indicated by colorimetric changes in a $\mathrm{pH}$ indicator), hydrogen sulfide (indicated by transformation of lead acetate to lead sulfide), and exopolysaccharide, all activities which have been previously associated with metal corrosion (Fig 2). Isolates demonstrating these activities have been further characterized by 
measuring the extent of these activities under varying growth conditions.

Acid production has been found to be highly dependent on the presence of glucose in the media (Fig 3). However glucose-affected alteration of $\mathrm{pH}$ may be mitigated by the presence of peptone. Presently, the production of hydrogen sulfide is being similarly analyzed using colorimetric assays. Preliminary results show that hydrogen sulfide production is significant under anaerobic conditions (Fig. 4). These studies indicate the conditions requisite for metal corrosion by repository bacteria. They also indicate ideal conditions for accelerated testing of candidate alloys for their resistance to MIC.

Corrosion cells, containing candidate waste package alloys and Yucca Mountain bacteria demonstrating the highest levels of MIC-associated activities, will shortly be constructed (Fig. 5). These will be monitored using electrochemical means, to determine the resistance of candidate waste package metals to MIC under conditions anticipated in the repository after waste deposition. 


\section{Summary}

The U.S. Department of Energy is currently conducting feasibility studies to assess the long term performance of a potential geological repository at Yucca Mountain, Nevada. Microbial growth of both native and introduced bacteria in the potential repository site may jeopardize the integrity of geologic nuclear waste disposal facilities by directly promoting corrosion of repository components and waste package materials, therefore a characterization of microbial effects on the repository site at Yucca Mountain is being perform. Acid producing bacteria, hydrogen sulfide producing bacteria and exopolisaccharides producing bacteria are activities demonstrated by the isolates retrieved from the $\mathrm{YM}$ and also activities associated with microbial corrosion. Further characterization of this behavior will be study. Corrosion cells, containing candidate waste package alloys and YM bacteria demonstrating the highest levels of MIC-associated activities, are being constructed. This will be monitored using electrochemical means, to determine the resistance of candidate waste package metals to MIC under conditions anticipated in the repository after waste disposition. 


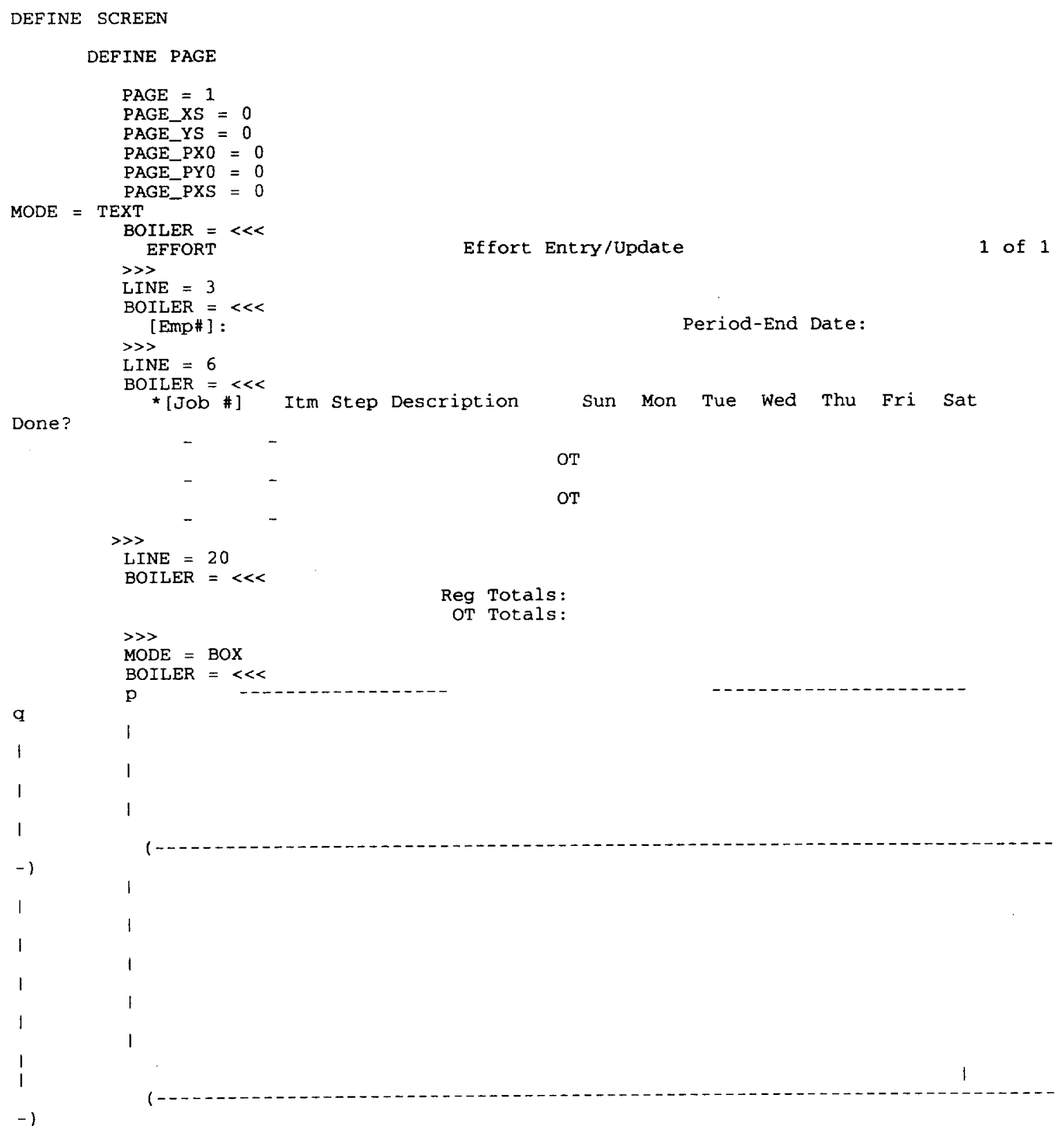

Figure 6: Sample code -- screen definition of character-based form, in particular Time Card. 


\subsection{Work Involved in Conversion}

Oracle Forms 4.5 was used to convert from character-based presentations to graphical user interfaces (GUI). See Figures $7 \& 8$ for sample converted GUI forms. Figure 6 is equivalent to Figure 1. Figure 7 is the GUI version of Figure 4.

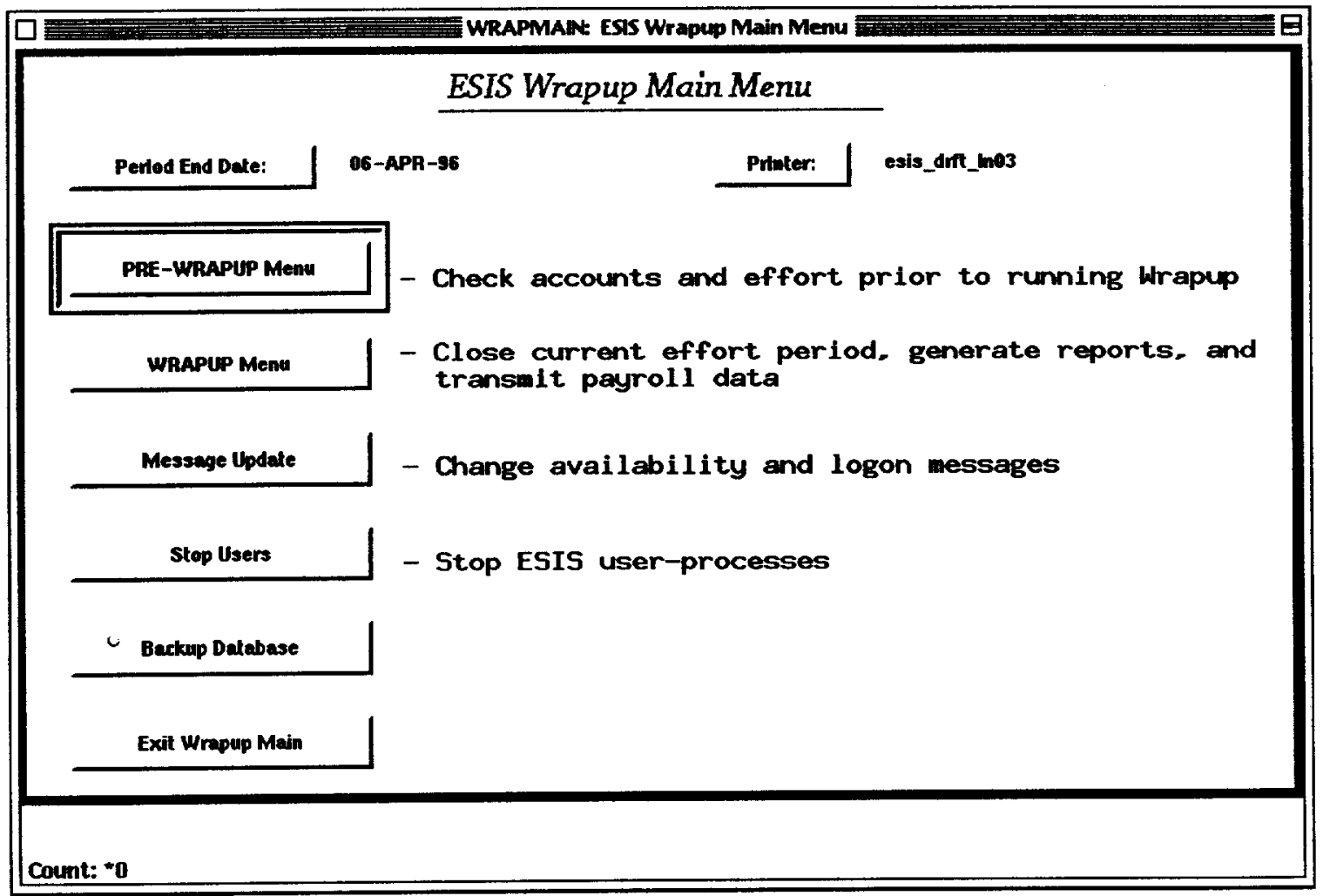

Figure 7: Converted menu system with graphical interface 


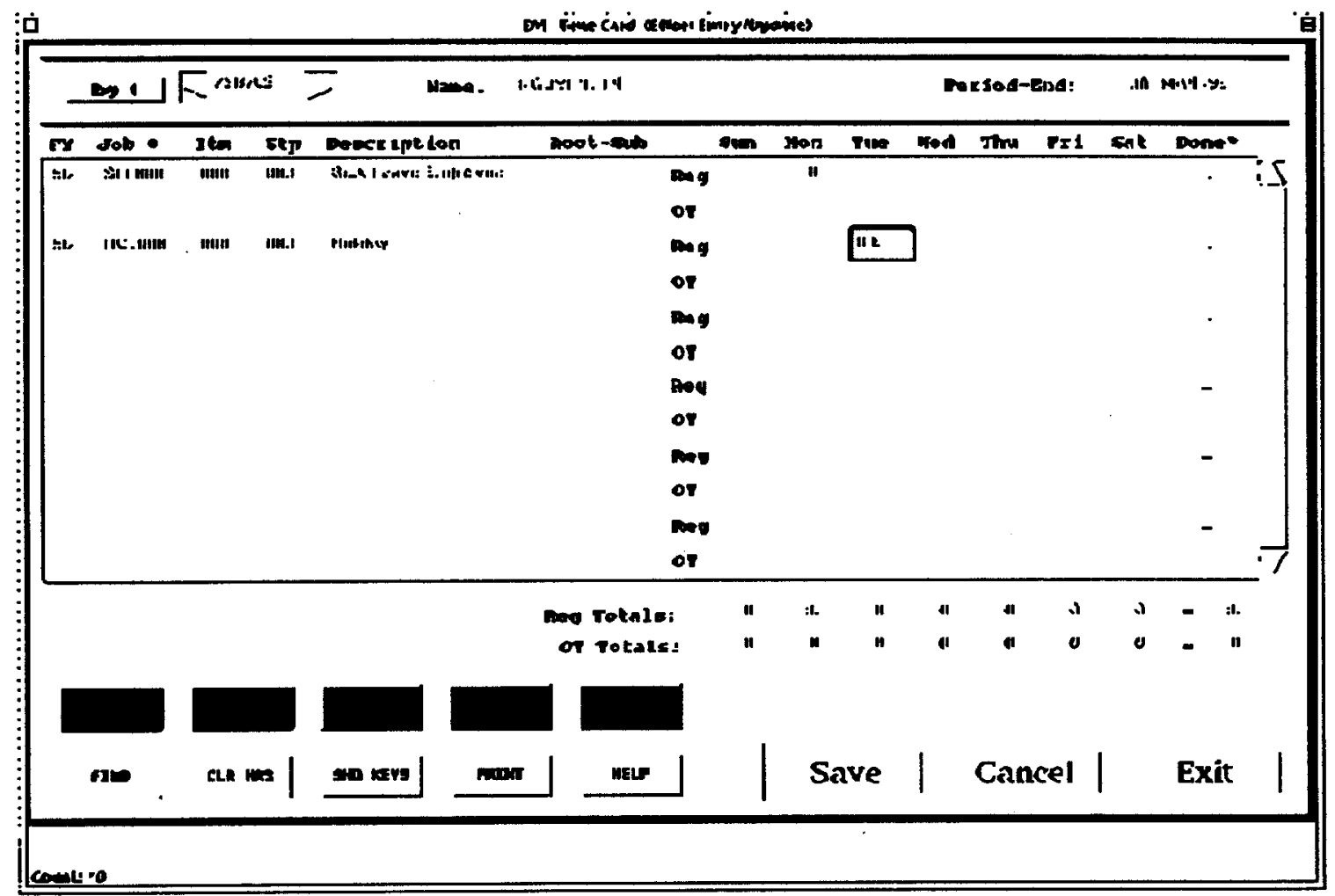

Figure 8: Converted menu system with graphical interface 
Forms 4.5 provides a generator, which translates text files such as those in Figures $5 \& 6$ to binary files such as those in Figures $9 \& 10$.

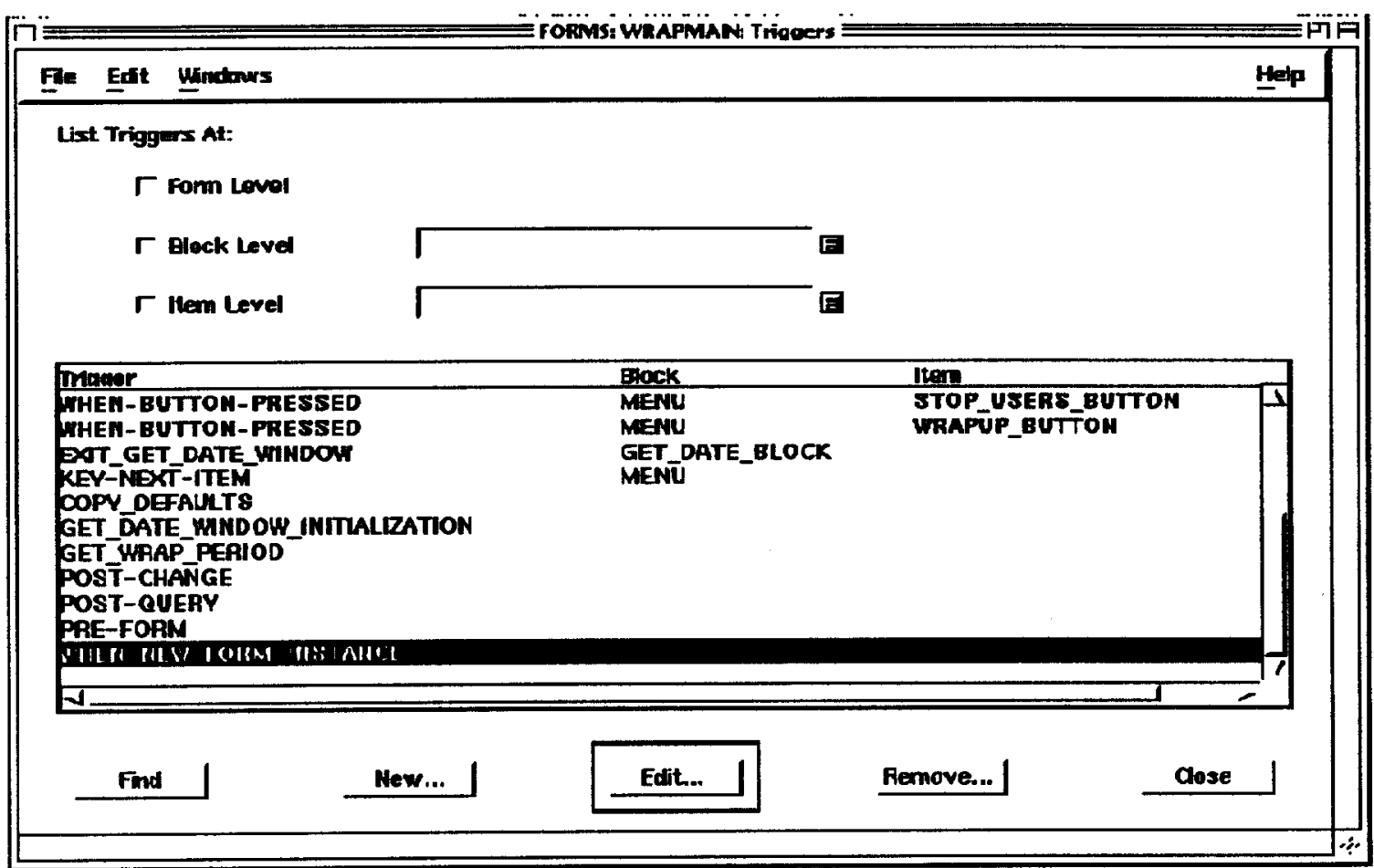

Figure 9: Converted source file of form after running the generator -triggers 


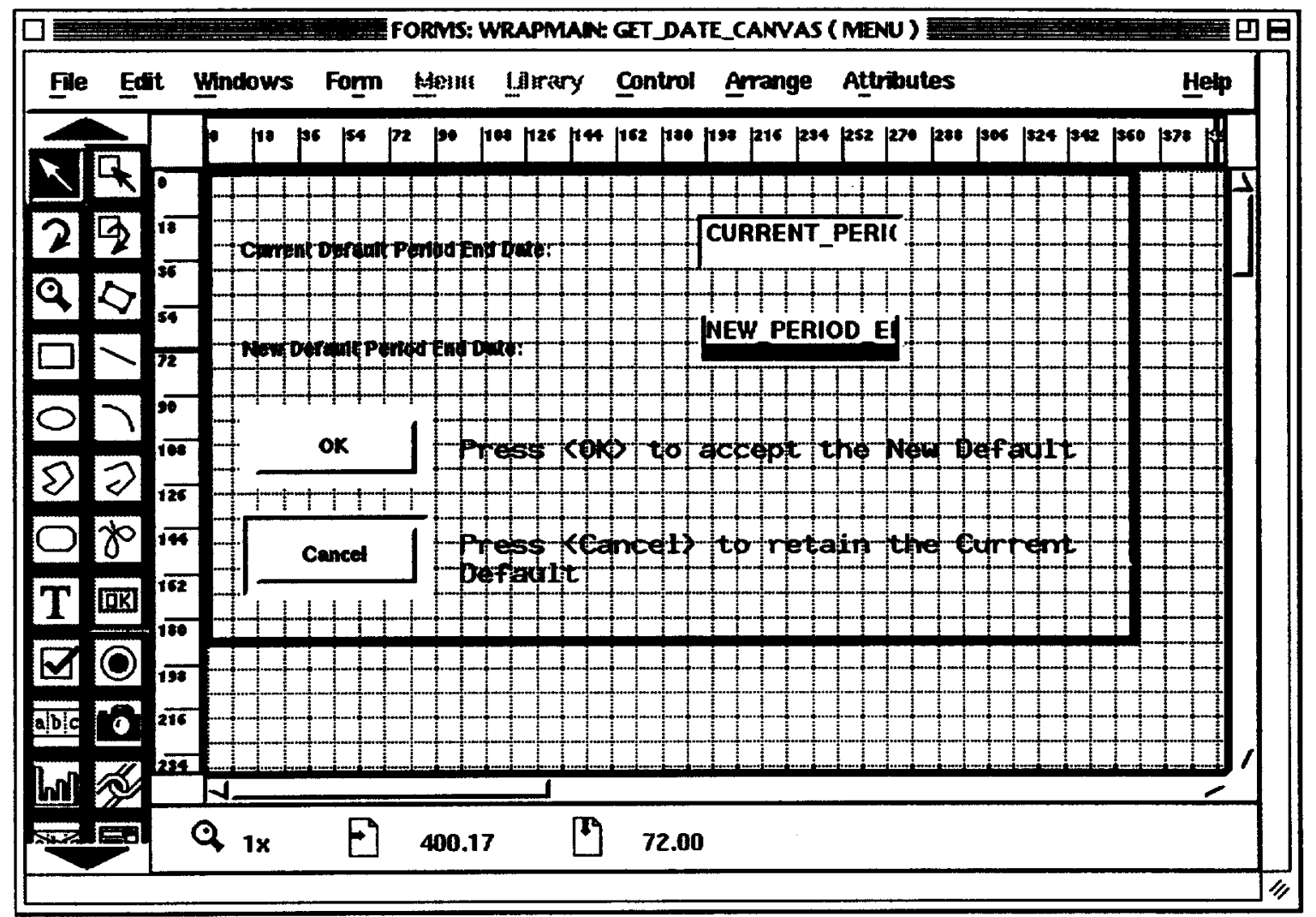

Figure 10: Converted source file of form after running the generator -canvas 
However, conversion from character interface to GUI is more involved than translation. All of the translated items and objects had to be modified. They had to be rearranged and repainted to conform to a GUI style of presentation. The conversion process also forced modifications to the underlying codes. For example, GOTOs and labels, such as those in Figure 5, were removed. The programming and coding styles were cleaned up. Calls to 'extinct' subprograms were replace with calls to valid ones. The most time-consuming change was modifying the navigational flow and processing model. For example, with a $\mathrm{CB}$ form, the programmer can severely restrict the user's navigation between fields, because the user can only use the keyboard, esp. tab, for navigation. With a GUI form, the user can navigate anywhere by clicking and focusing on any display item. See Figure 11 for an example of the modifications after the Oracle generator is run on the binary source file. 


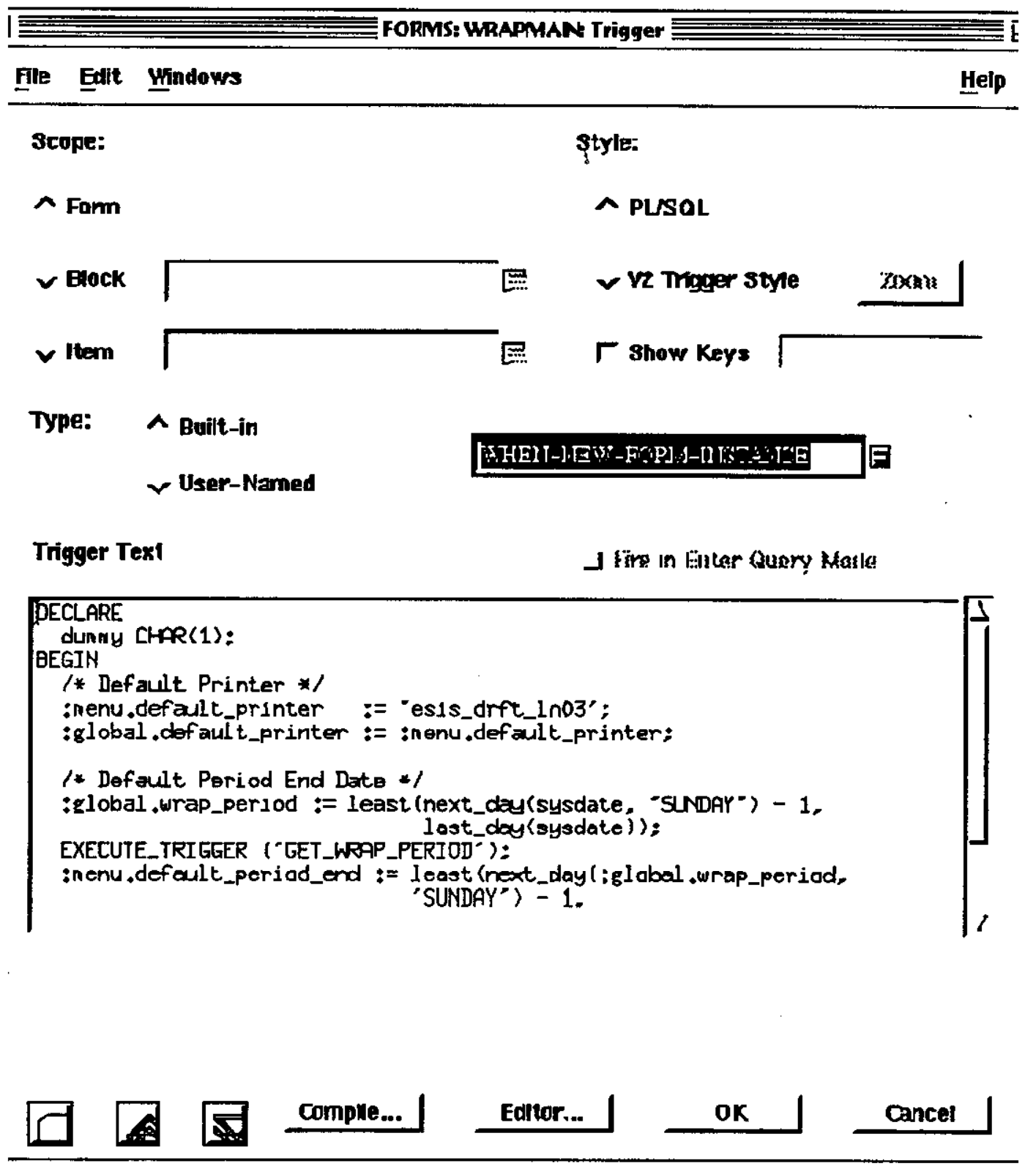

Figure 11: Modification of converted source file .- trigger; compare to figure 5 


\section{Results \& Discussion}

\subsection{Accomplishments}

After conversion, the new forms were tested on a development database and on 'live data'. For release, shell scripts were created to simulate a 'login' environment and version control, since the user no longer just 'telnets' into a server machine.

The new GUI forms are obviously easier to use than the character based ones. For example, the user can navigate between forms and between display items by clicking and focusing with the mouse. The GUI forms are easier to modify for the developers. For example, the visual attributes of a display items can be quickly changed by setting the properties.

At this point in time, only two modules of forms: Time Card and Wrapup Menu, have been converted. Conversion of the forms for job, step, and item entries and updates was start but not completed yet.

\subsection{Benefits \& Disadvantages}

The new GUI forms are easier to use and maintain. However, there are some disadvantages. To the end user, the forms are slower than the CB forms, because all of the display and windowing information are transmitted across the network. The runtime 'speed' of the forms to the end user is 'bottle-necked' by the network but not by the server machine. For the designer, triggers and attributes could distributed and embedded in many objects and display items.

The forms are maintained in one central location/server machine. The forms can be viewed by different platforms with $\mathrm{X}$ Window display managers. For example, the forms have been displayed on DEC and Sun workstations and Macintosh computers. However, the forms do not take advantage of native features on the desktop computers. The forms 
do not take advantage of the desktop computers' processors and memory by unloading the some of the processing and validations onto the desktop computers.

\subsection{Next Steps}

Currently, conversion of the character-based forms has been halted, because the customers want the errors and bugs in the scripts and report generators to be fixed before work continued. After ESIS is stabilized, then the conversion process will proceed. It seems that ESIS will not customize the forms for each target platform as discussed in the previous section. Instead, only one cross-platform front-end will be produced, and that single front-end seems to be a web page. So, probably within one or two years, the frontend of ESIS will be accessible through a web browser. 


\section{Bibliography}

Roberts, Christopher Getting Started with Oracle Power Objects, Version 1.0 Redwood City, California:Oracle Corporation 1995 p. 27 \& 37 


\title{
Developing a Finite Element Model for the Carpo-metacarpal Joint of the Thumb
}

\author{
By: \\ Jonathan Pearlman \\ Northern Arizona University department of Mechanical Engineering \\ Flagstaff Arizona \\ e-mail: pearlman@redhook.llnl.gov \\ Mentor: \\ Karin Hollerbach \\ Institute for Scientific Computing Research \\ Lawerence Livermore National Laboratory
}

June 6, 1996 


\section{Abstract:}

Developing a finite element model of the carpo-metacarpal (CMC) joint of the thumb provides essential information for designing anatomically correct and reliable prosthetic joint implants. Using CT scan data of a cadaver hand, a finite element model (FEM) was developed of the Metacarpal and Trapezium bones. This model, with anatomically correct orientation and physiological loading, will educate biomechanics researchers in the kinetics and kinematics of the joint and aid in developing a more reliable joint implant.

\section{Introduction:}

Osteoarthritis can plague many joints in the body, debilitating the person with severe pain. Osteoarthritis most commonly affects the thumb CMC joint; extreme forces on the articular surfaces of the metacarpal and trapezium bones can cause severe pain. Giurintano et. al.[1] have calculated these forces to be between six and twenty-four times the applied load depending on the grasp used. Implants must be designed to handle frequent loads of this degree for a sustained amount of time in normal, daily grasping activities, with little or no wear. In osteoarthritis, the joint surfaces wear, and a joint replacement operation, where a prostheric joint implant is inserted, is frequently required. Anatomically correct design and orientation of the im- plant in surgery will preserve normal movement and limit these stresses. Failures such as dislocations, high rates of material wear, and implant cement failures very frequently lead to additional operations. To date, CMC joint implants have had low success rates for a variety of reasons including implant orientation problems and inadequate design. Correcting these problems depends both on the design and the surgeon's ability to install the implant correctly.

Developing a computational model of the CMC joint allows for a computer aided design approach rather than lengthy and painful laboratory and clinical trials. By applying physiological loading boundary conditions to the bone meshes, finite element analysis (FEA) results reveal articular surface stresses needed in choosing implant geometry and material models with the least wear. Testing implants with the identical loading provides failure mode analysis helpful in implant design and comparison. FEA results have proven to be consistent with clinical trials when done on existing prosthetic implants, providing confident information of the validity of this method[2].

\section{Methods:}

A high resolution CT scan of a female cadaver hand was taken on site by NonDestructive Engineering (NDE) at Lawerence Livermore National Laboratory 
(LLNL.) With an isotropic resolution of 150 microns bone surfaces were well defined, and there were some outlines of tendons. Automatic segmentation and 2-D manual correction were performed using VISU, a program developed by Pierre-Louis Bossart at NDE. VISU develops boundaries for a given threshold range of each image. Final output are binary images for each "slice" of the data set which are triangulated and separated using the Dicer code, which implements the Marching Cubes algorithm[3]. These resulting surfaces (one for each bone) are specified in the off format which is readable by many visualization packages including Geomview. OFF files are then converted into point coordinate and connectivity files for import into the mesh generator.

The volumetric finite element mesh of the thumb metacarpal and trapezium were developed using TrueGrid ${ }^{T M}$ (XYZ Scientific Applications, Inc, Livermore, CA), a hexahedral finite element mesh generator capable of batch and interactive modes. Hexahedral elements, although more time consuming to produce, were chosen because of their increased precision over tetrahedral elements. TrueGrid ${ }^{T M}$ is able to read in the surface files and, through a long process of interactive commands, a mesh is generated and projected to the bone surface. Commands are then saved into an ascii file for batch processing. Boundary conditions were applied in TrueGrid ${ }^{T M}$ and NIKE3D, a nonlinear, implicit, three-dimensional finite element code developed at LLNL was used for the analysis.

\section{Metacarpal Mesh Generation:}

The metacarpal bone was read into TrueGrid ${ }^{T M}$, translated to the origin and rotated to align the long axis of the bone with the $y$ axis. Mesh generation is easier at the global origin, but final analysis is done in the original bone orientation to preserve physiological positioning. Curves were drawn on the two articular surfaces of the bone; one around the head separating the articular surface from the shaft, and others crossing palmer-dorsal and ulnar-radial directions. These curves capture articular surface definition and are used in mesh projection prior to final projection to the surface(See Figure 1.)

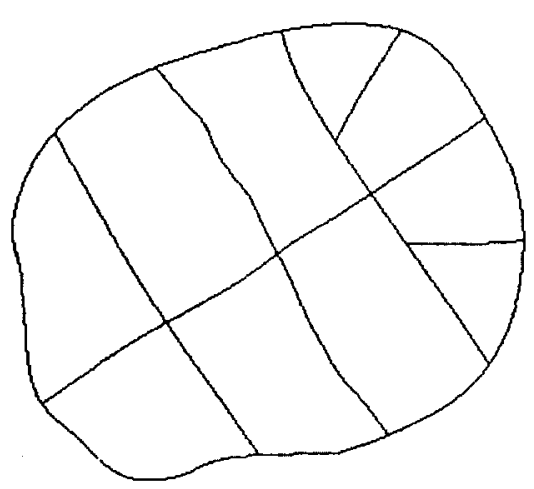

Figure 1: Articular surface curves (proximal)

The "butterfly" or "iron cross" technique, where the corners of the block 
are deleted and the mesh is stretched into a near cylindircal shape(See Figure 2,) was used to approximate the bone shape. This method helps prevent elements from having large angles (near 180 degrees) which can occur when projecting a cube onto a cylindrical shape. The mesh was then
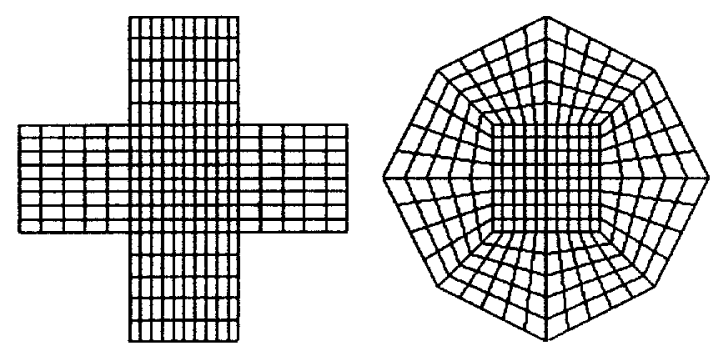

Figure 2: Cross section of Butterfly/Iron Cross technique

systematically expanded and projected to surface curves. Starting at the proximal end, the last set of elements were scaled, rotated, and translated near the surface before projection to the curves. The subsequent layers of elements were scaled and translated to intermediate positions down the shaft of the metacarpal. The distal end was meshed similar to the proximal with a final stage of curve projection to capture the articular surface. After final surface projection of the mesh, equipotential relaxation algorithms were applied on each face.

\section{Trapezium Mesh Generation:} The trapezium bone articulates with three bones in normal hand movement: the thumb metacarpal, trapezoid, and scaphoid. In this model attention was paid primarily to the surface articulating with the metacarpal, leaving a rectangular block to represent the rest of the bone. In initial stages of modeling the thumb CMC joint it is unnecessary and time consuming to model the whole trapezium; however, in future analysis runs it will be fully modeled. The initial steps in the meshing process were similar to the metacarpal: the surface definition was imported and translated to the origin and the desired articular surface was rotated parallel to the $\mathrm{xz}$ plane. Surface curves were drawn, the primary one around the perimeter of the asymmetrical saddle shaped surface and more crossing the saddle. The block was developed and the iron cross technique was applied to prevent abnormal elements. Nodes were then projected to the defined curves to approximate the surface. Final surface projection and equipotential relaxation produced a smooth, anatomically accurate model-for analyzing.

\section{Finite Element Analysis:}

Boundary conditions were applied in TrueGrid $^{T M}$ for two static situations. Both simulations used an axial (along the axis of the bone) displacement of the metacarpal with the trapezium fully constrained. In the first case the metacarpal was modeled as a rigid body and the trapezium as a soft polyethylene material. The second case was run with 
both bones non-rigid polyethylene. In each case NIKE3D converged and stress was apparent throughout the trapezium mesh.

\section{Conclusions}

Beginning with a cadaver hand, a model was developed which represents anatomical shape and positioning of the thumb CMC joint. As an initial step of computational modeling, this work shows the possibility of simulating accurate biomechanic systems for computer analysis. This model, with the inclusion of ligaments and physiological loads acquired from code written by Dave Guirintano (Gillis W. Long Hansen's Disease Center, Carville, Louisiana,) will offer surgeons and biomechanic researchers a look into the stress of this joint. Efforts are also being made to complete this model and expand it to include the whole hand.

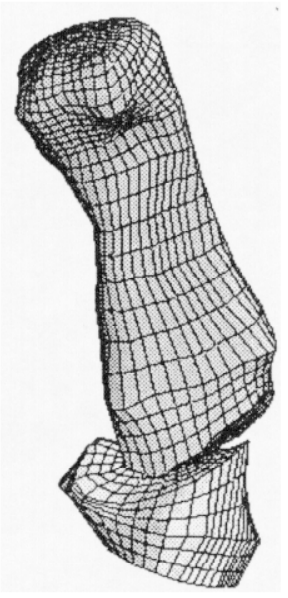

Figure 3: Complete Finite Element Model 


\section{References}

[1] A. Hollister D. Giurintano. A virtual five-link model of the thumb. 1994.

[2] Cheri Nielsen. Construction computational modesl for thumb carpometacarpal joint implants. SERS Research papers, 1995.

[3] W.E. Lorensen and H.E. Cline. Marching Cubes: a high resolution surface extraction algorithm. Computer Graphics, 21(3):163-169, 1987. 
Fig. 1

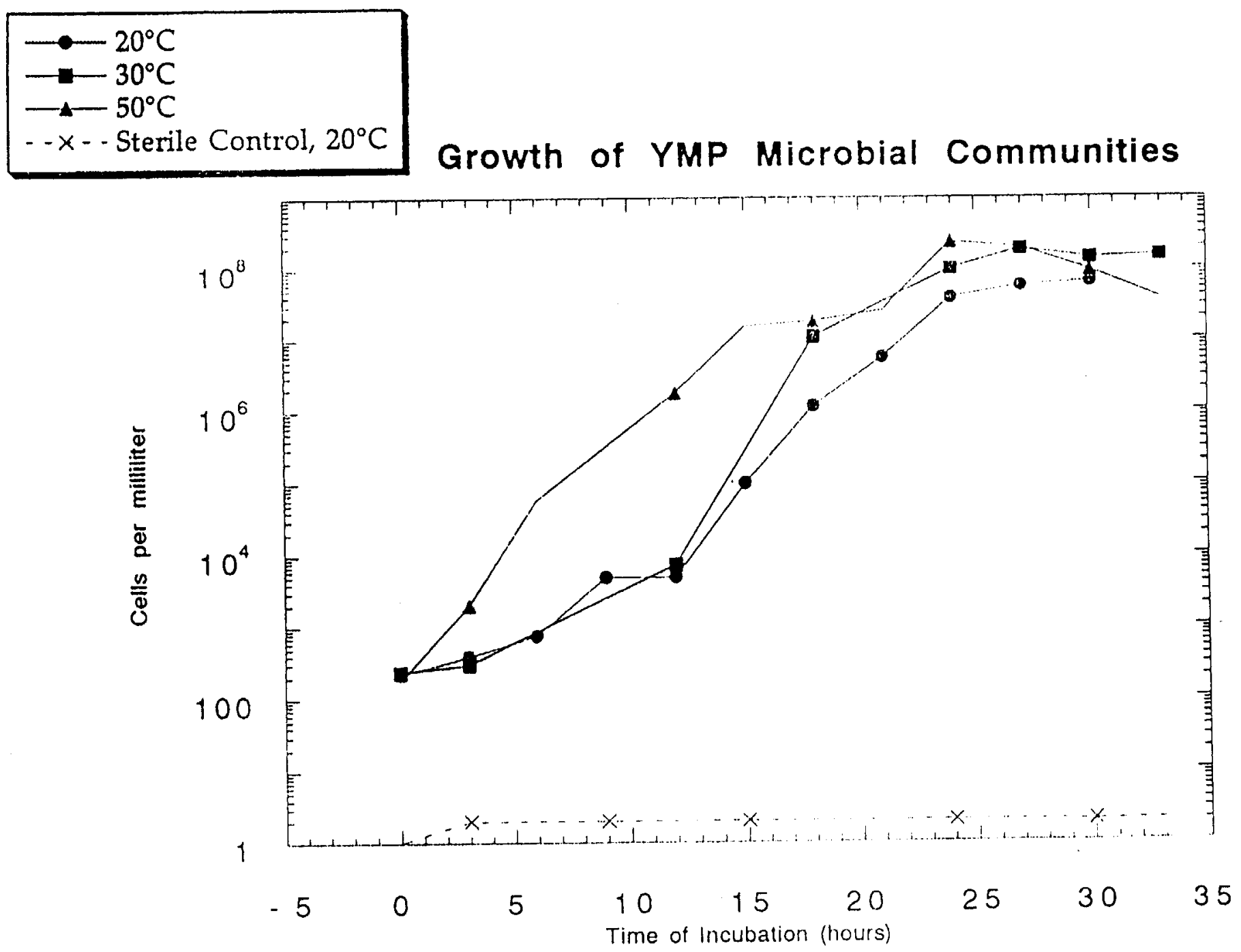




\section{Microbria contributions to metal corrosion.}

Fig. 2

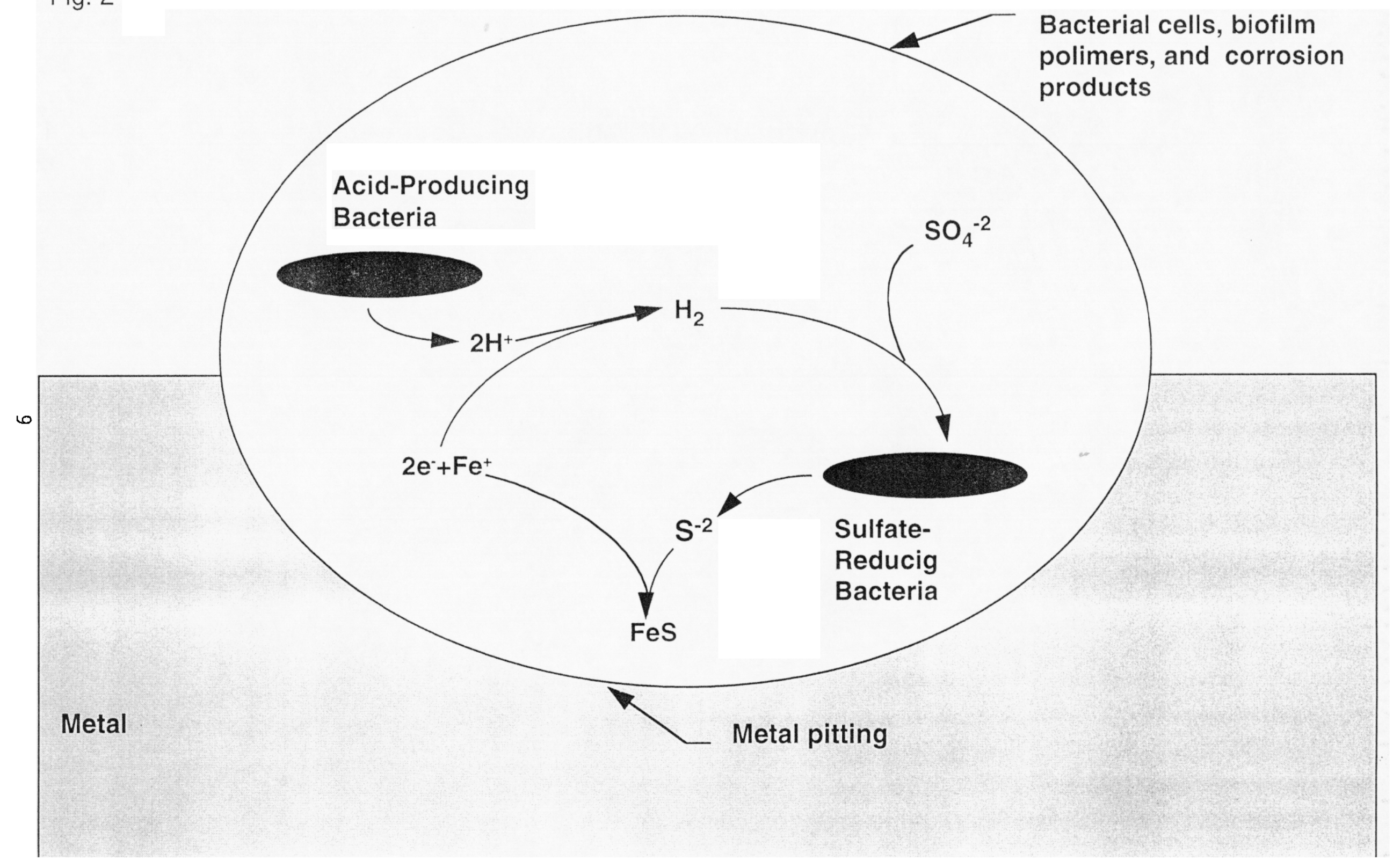


Fig. 3

STRING LB-71h-RT-13: Effect of Growth and Media on Alteration of $\mathrm{pH}$

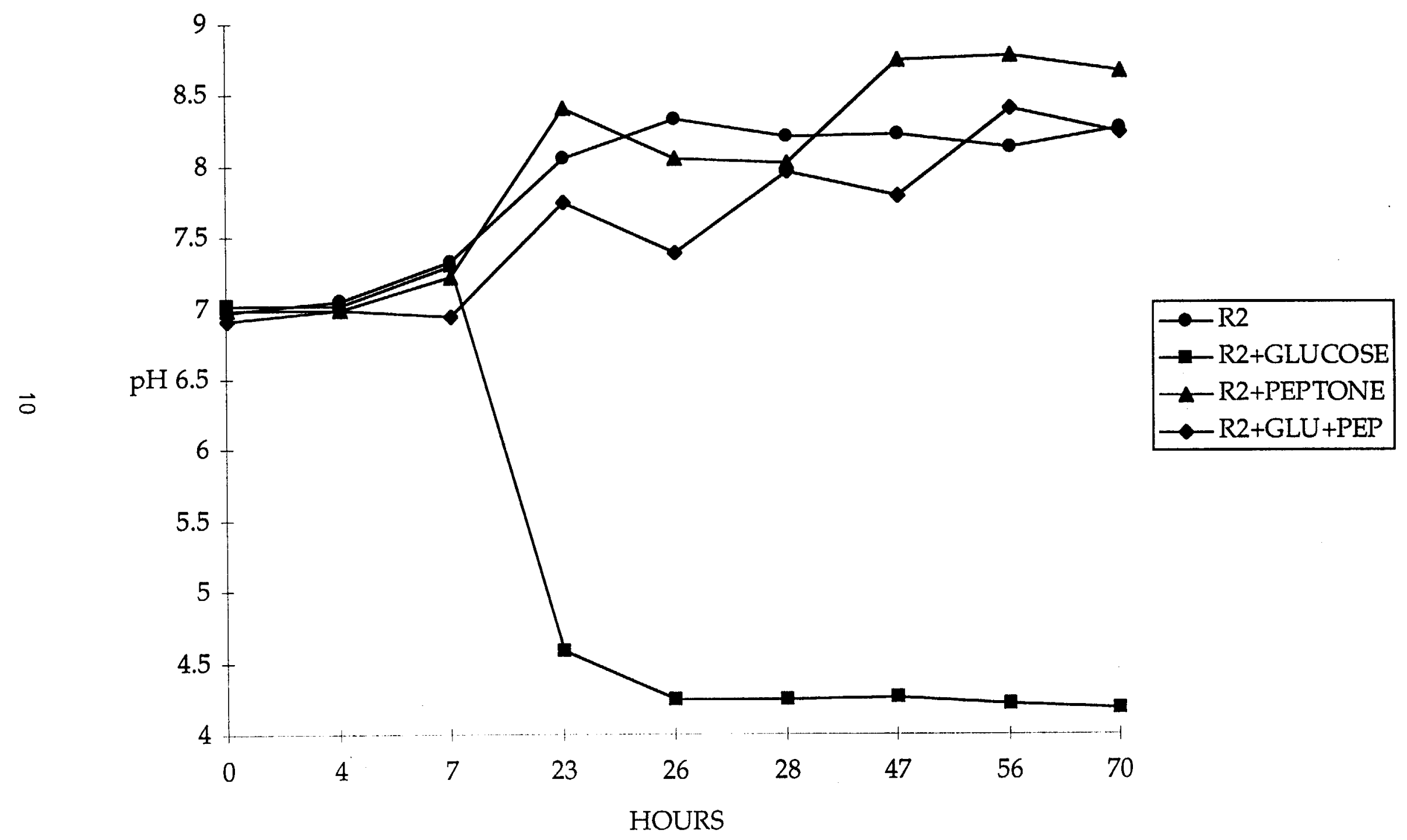


Fig. 4

Sulfide Generation by YMP Isolates

During Growth in $\mathrm{R} 2+$ pefione under Anaerobic Conditions

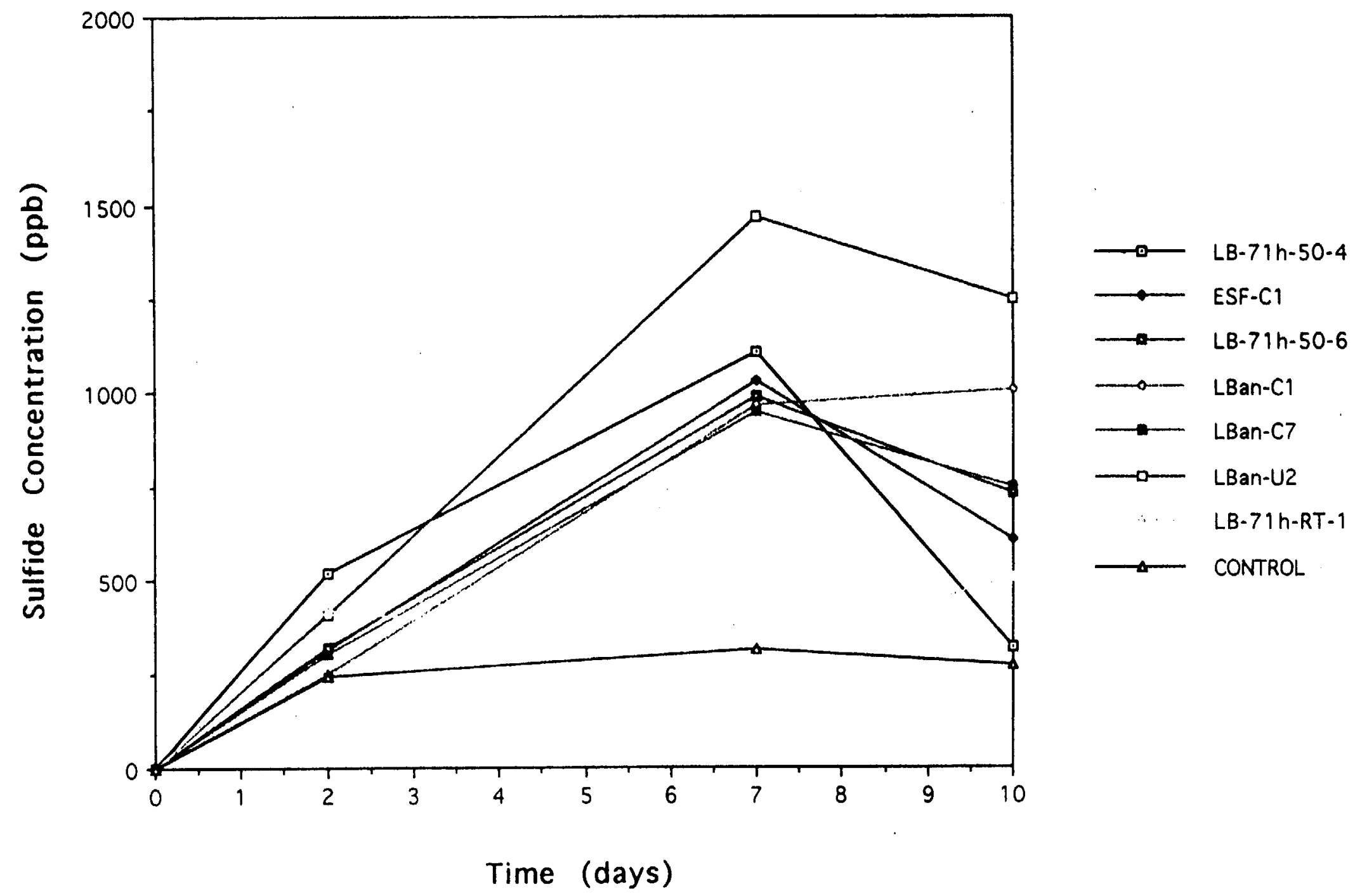


Fig. 5

\section{Electrochemical Cell for Measuring MIC Corrosion Rates}

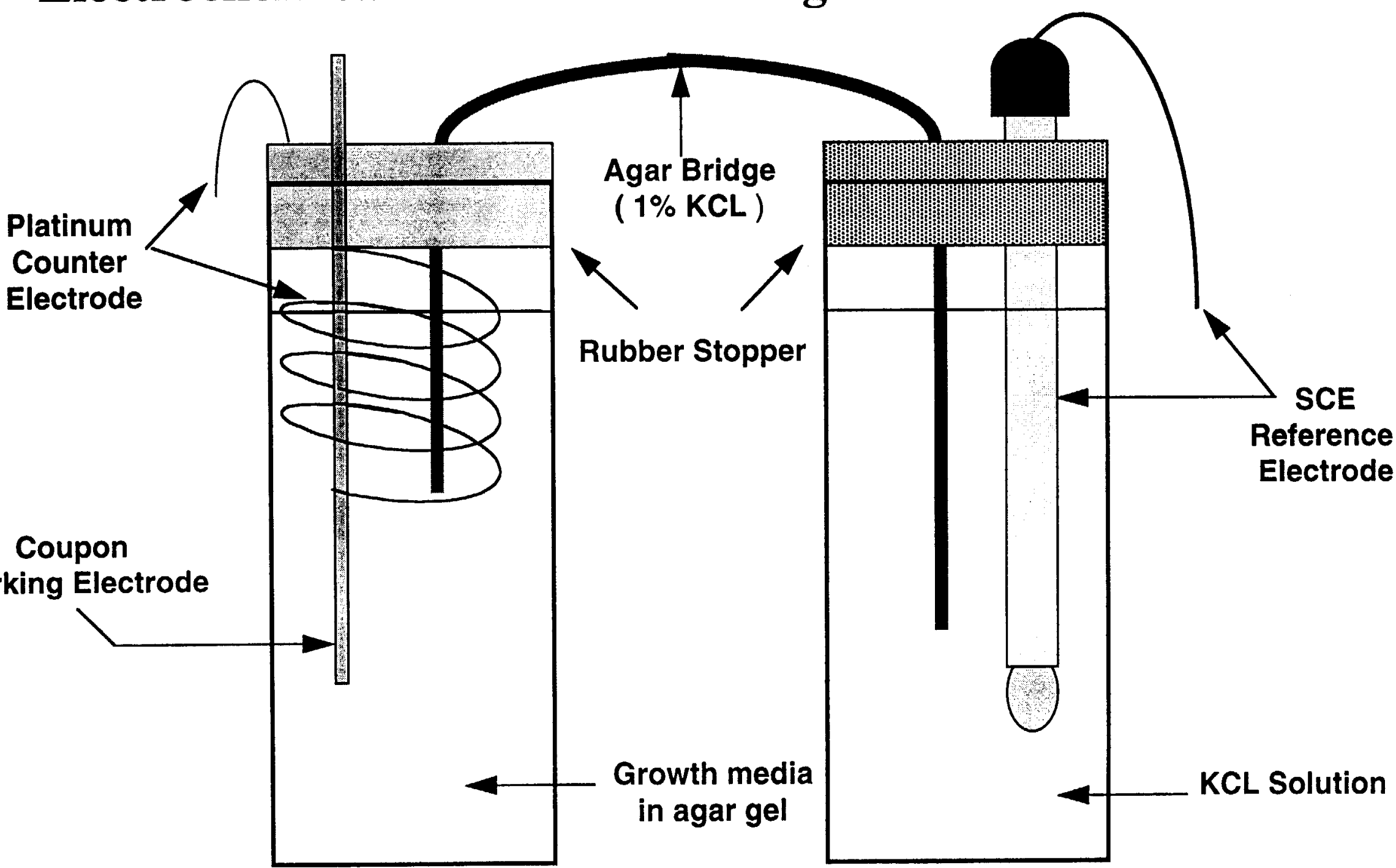




\section{References}

1. Geesey, G. and Cragonilo, G.A. (1993) A Review of the Potential for Microbially Influenced Corrosion of High-Level Nuclear Waste Containers. Nuclear Regulatory Commission, Center for Nuclear Waste Regulatory Analyses, Report No. CNWRA 93 - 014 Southwest Research Inst. , San Antonio, TX.

2. Horn, J.M. (1996) High Level Radioactive Waste Management Conference, Las Vegas, Nevada, April - 29 - 1996.

3. Horn, J. M. and Meike, A. (!995) Microbial activity at Yucca Mountain. Lawrence Livermore Laboratory Report UCRL-ID-122256.

4. Horn, J.M. and Meike A. (1996) A Program to Assess Microbial Impacts on Nuclear Waste Containment. Lawrence Livermore Report UCRL- JC122732.

5. Jones, D. A. and Penny, A.J. (1995) Microbiologically Influenced Corrosion. Status Report. 


\title{
Isolation of full-length cDNA's using the New System GeneTrapper
}

\author{
James D. Sparks \\ Grambling State University
}

\section{Lawrence Livermore National Laboratory \\ Livermore, CA 94550}

$$
\text { May 10, } 1996
$$

Prepared in partial fulfillment of the requirements of the Science and Research Semester under the direction of Wufang Fan, Greg Lennon research mentors in Lawrence Livermore National Lab 


\section{Abstract \\ Isolation of Full-length cDNA's using the new system GeneTrapper}

An exon was identified by genomic sequencing from the human chromosome 19 q13.1 loci. Sequence analysis showed that it is homologous to rat neurocan.(Rauch et al., 1992: 1995) Northern analysis demonstrated that the gene is expressed only in brain tissue. The size of the gene is approximately $6.8 \mathrm{~kb}$ in length. We designed two primers based on the sequence data from the cDNA fragment. These Primers were used with the GeneTrapper protocol to isolate a full-length cDNA from a human brain cDNA library.

The results showed that the trapped cDNA's contained about 5\% of the clones, which were shown to be positive by PCR for the cDNA insert, when using the neurocan primers. Mini prep and restriction digest showed the longest insert to be $5.1 \mathrm{~kb}$. Currently, work is progressing as new primers are being designed from the most recent 5' sequence. These new primers will be used in new rounds of GeneTrapper experiments.

Compared to conventional methods the GeneTrapper protocol is fast, powerful, compatible, and safe in the isolation of full-length cDNA's. 


\section{Introduction}

The study of the human genome is a fifteen year coordinated effort by many scientist around the world. This scientific endeavor touches upon many disciplines other than biology and genetics. The overall goal of the project is to generate a high resolution map of the 80 to 100,000 genes in the genome, showing there exact location and sequence.

Our group under the Lawrence Livermore National Lab Human Genome Center is a cDNA identification team. The goals of this group are to isolate, map, and sequence all the genes on chromosome 19. Our previous work has shown that we can easily isolate cDNA's from certain genomic regions using hybridization selection. These isolated cDNA's are usually in the form of fragments. The difference being fragments verses full-length cDNA is what our group is exploring. To isolate full-length cDNA's, the routine method used was filter- 


\section{me trom}

\section{Wethods}

'

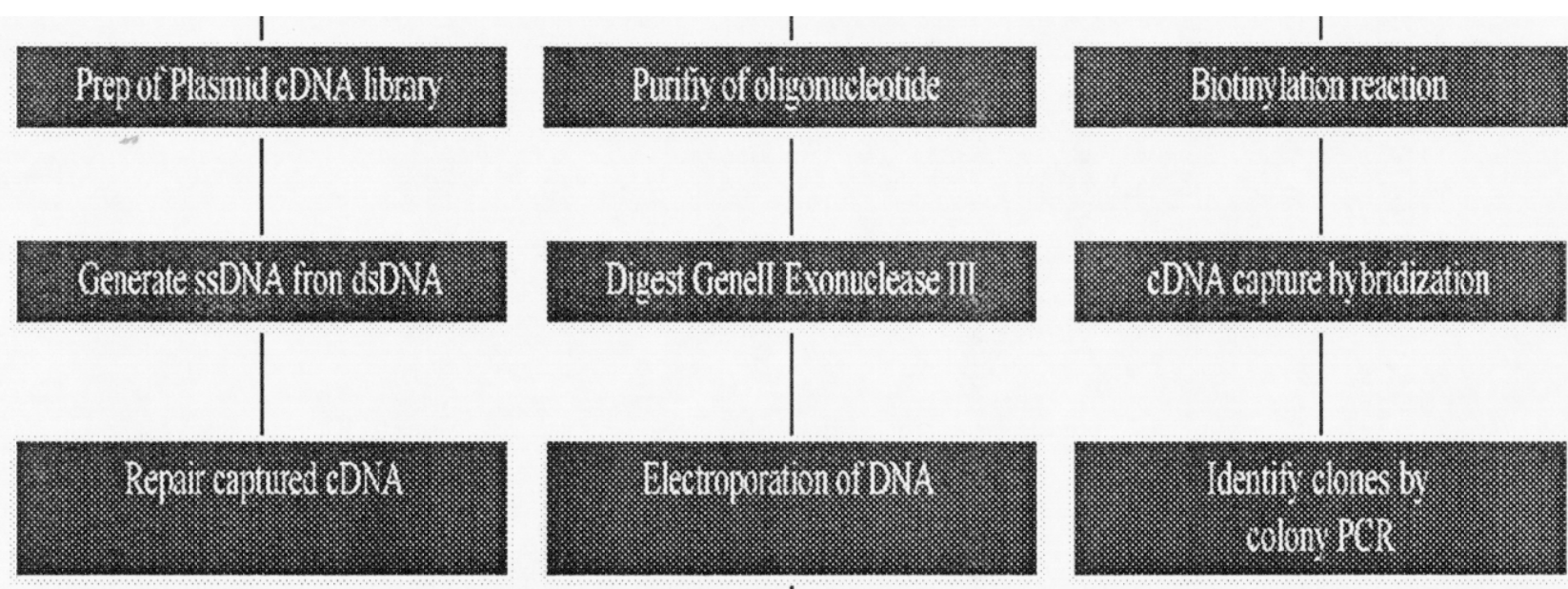

Wentifi done by colon livondization 
lifting. Normally this methods is slow, time consuming and nonproductive.

Recently, GeneTrapper, a new method was introduced to isolate full-length the full-length cDNA's. Knowing that many laboratories have tried this technique and failed, we adapted the method with some modification and used it to isolate human neurocan cDNA on chromosome 19. The results will show that GeneTrapper can be used successfully to isolate full-length cDNA's.

\section{Experimental Procedures: GeneTrapper}

The most popular methods of identifying cDNA clones generally are not as efficient as the GeneTrapper technique. One method such as in situ hybridization only generates clones at $10^{6}$, and it may take weeks, months or years for generation of the desired clones.

GeneTrapper (GIBCO-BRL) is a fast, powerful technique that allows for the capture of clones in days as compared to conventional methods. This system is so powerful it allows the user to generate select clones to $10^{12}$. This provides a better rate for full-length cDNA retrieval. Not only is GeneTrapper fast, it's flexible. We used it on a brain cDNA library(GibcoBRL), but it works well with most phagemid 
libraries as well. Most importantly, GeneTrapper uses no radioactive substances unlike in-situ hybridization.

\section{Target cDNA}

A exon homologous to rat neurocan (Rauch et al.,1992) was located on the $\mathrm{q}$ arm of chromosome 19 in the 13.1 loci. (See Attachment) Through genomic sequencing we designed two primers for the neurocan sequence. The primers were subjected to oligonucleotidal purification using the protocols of GeneTrapper. This aspect of the protocol includes assemblage of a $12 \%$ acrylamide gel consisting of $8 \mathrm{M}$ urea, $1 \mathrm{x}$ TBE buffer, TEMED, and ammonium persulfate. The primers were electrophoresed using a vertical gel box for $21 / 2$ hours 180 volts. Upon excision from the gel, the oligos were washed with $70 \%$ ethanol and dried at room temperature by vacuum centrifugation. A mass spectrophotometer set at an optical density $\left(O D_{260}\right)$ was done to determine concentration. Concentration of primer was recorded well above $0.5_{\mathrm{m} /} / \mathrm{ml}$ minimum. 


\section{Location of Neurocan cDNA}

- Homologous to mouse neurocan.

- Target exon located by genomic sequencing to chromosome 19p13.1 locus.

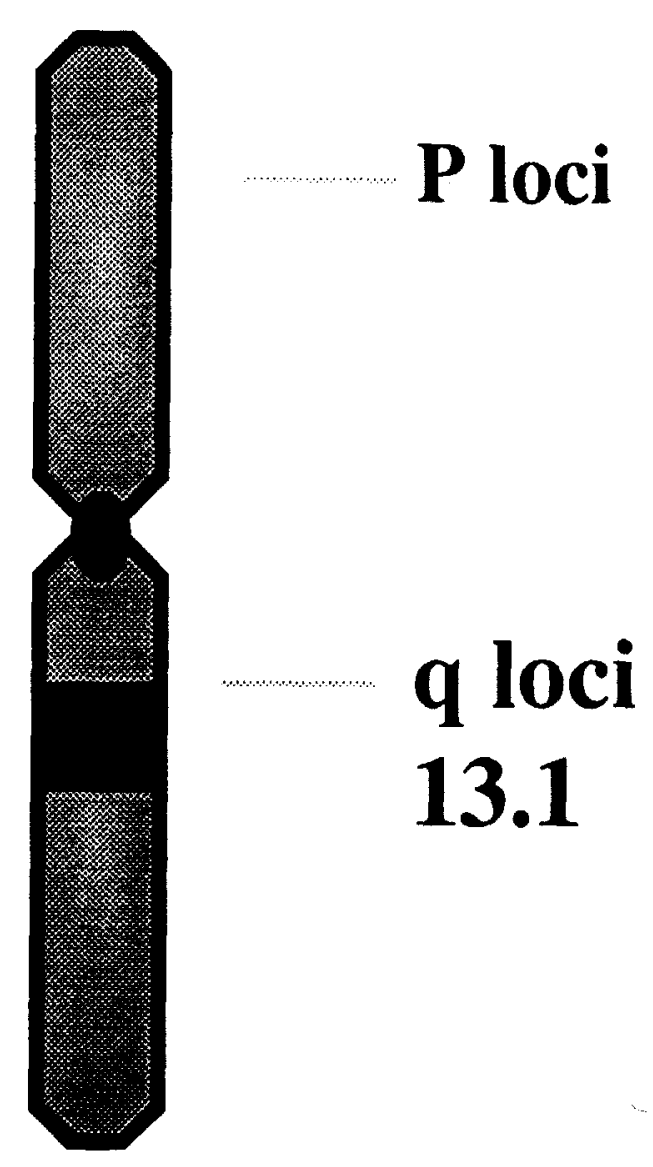




\section{Biotinylation of oligonucleotides}

In order to isolate a full-length cDNA we must be able to

remove the target inserts complimentary to the cDNA we want. By attaching a magnetically attractive substance called Biotin

dCTP(Gibco BRL) to our neurocan primers $3^{\prime}$ end using an enzyme called Terminal deoxynucleotidal Transferase (TdT), we can link our primers to the target DNA strand. Using a $16 \%$ acrylamide gel to determine extent of biotin labeling we can see that the Neu F1 R1 F2/R1 primers were labeled successfully.( See figure 2)

Neurocan - 1984 Oligos:

Neu-F1: 5' CGG CAG TCC TTC TCG GCA TCT T 3' (325-346 bp)

Neu-R1: 5' TTC TGG GAG GAG GTG GCA AGT T 3' (728-707bp)

Optimal annealing temperature : $60.20 \mathrm{C}$

PCR size: 404 bp

Neu - F2: 5' TGG GGA GGC AAA GGC AGA CAA A 3' (452-473bp)

Neu - R2: 5' TTC TGG GAG GAG GTG GCA AGT G 3' (728-707bp)

Optimal annealing temperature: 59.3 OC

PCR size: 277bp

Figure 2: Two neurocan primers used in biotin labeling. 


\section{Generation of ssDNA from dsDNA}

Another important procedure involves generating ssDNA from a dsDNA targets. The use of two enzymes are needed to generate our single strand of DNA. DNA supercoils that were generated from the brain library(Gibco BRL) in plasmid preparation were subjected to Gene II and Exonuclease III. These two enzymes when used in sequential order will reduce the dsDNA coil to a single strand of DNA. Gene II is a site specific endonuclease that binds and nicks the viral strand of the DNA supercoil. (see figure \#3) After nicking Exo III is used to digest the nicked strand from the $3^{\prime}$ end, leaving a covalently closed ssDNA strand. This is evident by the two bands shown in lane four of figure \#3. Now the ssDNA is ready for hybridization to the biotinylated neurocan primers.

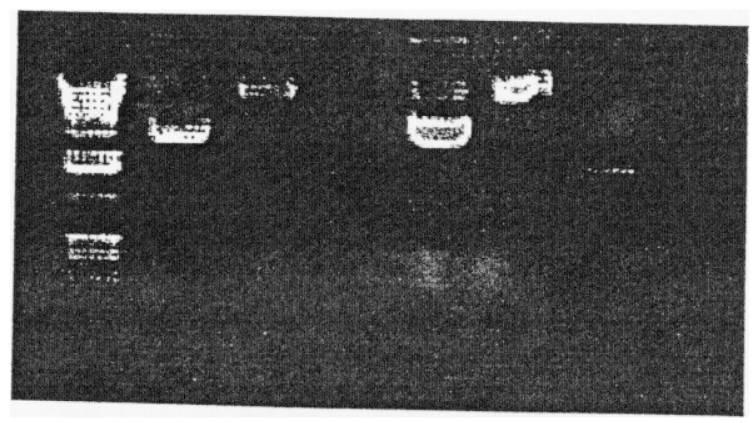

Figure \#3 Gene II Exo III digest 


\section{cDNA Capture by Hybridization}

The ssDNA from the brain library (Gibco BRL) that was generated by Gene II/Exo III is hybridizes to neurocan labeled oligos in a solution of $4 \mathrm{x}$ hybridization buffer(20mMTris-HCL (ph 8.0$), 1 \mathrm{mM}$ EDTA) This is done by denaturing the DNA at $95^{\circ} \mathrm{C}$, chilling on ice for $1 \mathrm{~min}$ and adding $1_{\mathrm{m} 1}$ (20ng) of biotinylated oligonucleotides. The reaction was placed at $37^{\circ} \mathrm{C}$ for 1 hour. The next step involved removal of labeled oligo-ssDNA hybrids.

\section{Isolation of Biotin-oligos and target ssDNA}

\section{Streptavidin Beads}

After hybridization, removal of the biotinylated oligos and target ssDNA is in order. To remove the target DNA the hybridized oligos and ssDNA are capture on magnetic beads coated with streptavidin. A magnetic attraction between biotin and the streptavidin allow this to take place. The beads will remove the target DNA from any non-hybridized ssDNA. The magnetic beads are then washed several times in a $1 x$ elution buffer(Gibco BRL) to remove the hybrids from magnetic beads. The results are pure 
target ssDNA clones that can be repaired and amplified using either the same primers or different oligos of choice

\section{Convert Isolated ssDNA to dsDNA}

To repair the ssDNA we prepared two mixtures. A repair mixture which uses; $10 \mathrm{x}$ repair buffer 1.5 ul $95^{\circ} \mathrm{C} 1 \mathrm{~min}$ $10 \mathrm{mM}$ dNTPs $\quad .5 \mathrm{ul}$

Taq Repair Enz $\quad .5 \mathrm{ul}$

$\mathrm{H}_{2} \mathrm{O} \quad 12.5 \mathrm{ul}$

A DNA primer mixture was prepared in conjunction with the repair mix. It consisted of; $\mathrm{H}_{2} \mathrm{O} \quad 7.5 \mathrm{ul} 70^{\circ} \mathrm{C} 1 \mathrm{~min}$ $10 \mathrm{x}$ buffer $1.5 \mathrm{ul}$ Captured DNA 5 ul Oligos (unbiotin) $50 \mathrm{ng}$

Each solution was incubated at the required temperature. After which, the repair mixture was added to the DNA mixture in aliquots of $15 \mathrm{ul}$ and allowed to stand at $70^{\circ} \mathrm{C}$ for $15 \mathrm{~min}$. This will allow the primer extension to occur. The repaired DNA was precipitated with $70 \%$ ethanol and stored in $10 \mathrm{ul}$ of TE.

\section{Transformation of Target DNA to Escherichia coli}

Upon repair of DNA from cDNA library (Gibco BRL) and neurocan designed primes introduction into a host for replication was needed. Only by placing in a host can the target clone be amplified. 
To transformed the DNA we used DH12S competent cells from Gibco BRL. 40 ul of transformable cells were used in conjunction with $2 \mathrm{ul}$ of repaired DNA. the mixture was placed in a Bio-Rad GenePulser and electroporated at $1.8 \mathrm{kv}$ at $200 \mathrm{ohm}$ for two seconds.

After electroporation $1 \mathrm{ml}$ of S.O.C. medium was added to the cells. The DH12S cells were incubated on a shaker at $37^{\circ} \mathrm{C}$ for $1 \mathrm{hr}$. Luria Broth plates $\left(950 \mathrm{ml} \mathrm{H}_{2} \mathrm{o}, 10 \mathrm{~g}\right.$ bacto-tryptone, $5 \mathrm{~g}$ bacto-yeast extract, $10 \mathrm{~g} \mathrm{NaCL}, 100 \mathrm{mg} / \mathrm{ml}$ ampicillin) were prepared earlier. Samples of the transformed cells were added to each plate in $100 \mathrm{ml}, 200 \mathrm{ml}$, and $400 \mathrm{ml}$ amounts. Each plate was labeled according to each primer that was designed, (Neu F1/R1, Neu F1/R2) and place in $37^{\circ} \mathrm{C}$ overnight.

\section{PCR, Mini-prep and Restriction digest}

We removed the LB plate plates containing the target clones from the incubator and proceeded to test for positive clones by randomly choosing clones from the plates and performing a Polymerase Chain Reaction (PCR). After PCR the DNA was run on a $2 \%$ agarose gel to determine the percentage of positive clones. The results were $78 \%$ positive for clones of neurocan inserts.

Positive clones were spotted to fresh LB plates and numbered accordingly. These plates were grown overnight and a PCR was done 
using the Neurocan primers to amplify the DNA. For every 100 clones used in the PCR process, we got 5 or 6 positive clones of interest. A sample of the positive was taken directly from the plate and inoculated to a new test tube containing $4 \mathrm{ml}$ of Terrific Broth. These tubes were incubated overnight on shaker at $37^{\circ} \mathrm{C}$. Using a Promega DNA purification kit, the cells containing our neurocan insert were lysed and the DNA removed.

A 20 ul restriction digest was performed on the purified DNA to determine the insert length. For the digest we used 13 ul $\mathrm{H}_{2} \mathrm{O}, 1$ ul NOT I, 1 ul SAL I, 2 ul $10 \mathrm{H}$ Buffer(Boehringer), 3 ul DNA. The mixture was allowed to incubate for 1 hour. A $1 \%$ agarose gel (Gibco BRL) was ran using a $1 \mathrm{~kb}$ ladder as a measurement. The results showed a Neurocan insert of $5.1 \mathrm{~kb}$. (see figure 4)

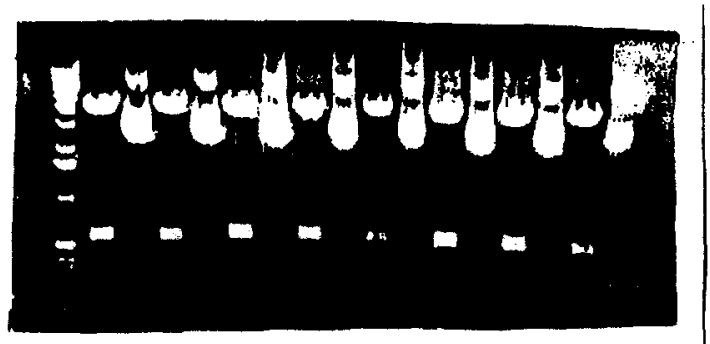

Figure 4. $1 \%$ Agarose gel showing the neurocan $5.1 \mathrm{~kb}$ insert. Lane 1 is $1 \mathrm{~kb}$ ladder

\section{Discussion of Results}


A $5.1 \mathrm{~kb}$ full-length cDNA of human neurocan was isolated using the GeneTrapper system. This isolation was not without problems. In attempts to verify the 5.1 insert many secondary experiments revealed different insert sizes for the same gene. The $5.1 \mathrm{~kb}$ full-length cDNA was transferred to $\mathrm{DH} \propto 5$ cells from DH12S cells. These cell were incubated at $30 \mathrm{OC}$, lysed and subjected to restriction digest using NOT1/SAL1 . The results showed that the gene was indeed a $5.1 \mathrm{~kb}$ insert. However, we cannot understand why recombination occurred in the DH12S cells. When the cells were grown at $370 \mathrm{C}$, they were not very dense and there was very little DNA present in the DH12S cells. One explanation is that he gene may be toxic to its host.

Isolation of this full-length cDNA has proven that GeneTrapper is an efficient method to work with. The results that we achieved in two weeks could have taken months or years using conventional methods. Currently, new primers have been designed from the most recent 5' end of the neurocan cDNA for new rounds of GeneTrapper experiments.

\section{S u m m a r y}




\section{Su m mary}

A human neurocan gene of $6.8 \mathrm{~kb}$ was isolate on chromosome 19 q13.1. Nothern analysis revealed that the tissue expression is limited to brain tissue. We designed two primers from genomic mRNA to be used in a new system called GeneTrapper. Our goal was to isolate a full-length cDNA that is complimentary to the human neurocan gene. GeneTrapper provide us with neccessary clone to isolate $5.1 \mathrm{~kb}$ of the $6.8 \mathrm{~kb}$ gene. Conventional methods may have taken monhs or even years to achieve these results. With some modificaton, GeneTrapper provided a means to achieve positive results in a two week period. 


\section{Bibliography}

Rauch U., Karthikeyan L., Maurel P., Margolis R.U., Margolis R.K.

(1992). Cloning and Primary Structure of Neurocan, a

Developmentally Regulated, Aggregating Chondroitin Sulfate

Proteoglycan of Brain. Journal of Biological Chemistry.

$267: 19536-19547$

Rauch U., Grimpe B., Kulbe G., Arnold-Ammer I., Beier D.R., Fassler R., (1995). Structure and Chromosomal Localization of the Mouse

Neurocan Gene. Genomics. 28: $405-410$ 


\title{
A SWMM Model of the Storm Drain System at Lawrence Livermore National Laboratory
}

\author{
Louise L. Wells \\ Operations and Regulatory Affairs Division \\ Mentor: Erich Brandstetter
}

Science and Engineering Research Semester Spring 1996 


\begin{abstract}
:
The objective of this project is to create a working model of the storm drain system at Lawrence Livermore National Laboratory (LLNL) using the USEPA Stormwater Management Model (SWMM). Once complete, the model will aid in storm water monitoring by creating plots of flow and pollutant loading versus time at different points in the storm drain system. Half of the work on this model has been invested in creating an updated map of the storm drain system, which is necessary for completion of the model, and the other half has been put into setting up the model. Currently, the SWMM model is still under development and is set up to receive more data about the storm drain system as it becomes available The updated storm drain map is not completed. Nevertheless, the model has been used to analyze a theoretical storm event and to estimate flow rates and the time required for flow to cross the site.
\end{abstract}




\section{Introduction:}

Developing the model of the storm drain system at Lawrence Livermore National Laboratory (LLNL) is an important part of understanding the infrastructure of LLNL. The storm drain system is a complex network of pipes that has resulted from fifty years of building and rebuilding. The storm drain model will aid in storm water monitoring efforts, evaluating best management practices, and will aid in the evaluation of the pipes and channels that make up the storm drain system. The United States Environmental Protection Agency's (USEPA) Stormwater Management Model (SWMM) is being used to create the model. Information about drainage areas, pipes and channels is needed for this model. A large portion of time was spent gathering this data for the model. At this point, only a portion of LLNL has been entered to the model. Results have been obtained at this time that give a good representation of the capabilities of the model.

\section{The Storm Drain System at LLNL}

Storm drain systems route storm water from the ground surface to pipes and channels. Figure 1 shows an incomplete map of the storm drain system at Lawrence Livermore National Laboratory. The map is currently under review and verification, therefore all of the data have not been entered. The storm drain system at LLNL is made up of 35 miles of conduits, 10 miles of open channel, 88 manholes, and 973 catchbasins. Storm water generally enters the storm drain system through catchbasins. Catchbasins are grates on the surface that collect runoff. Several pipes can meet at a single 


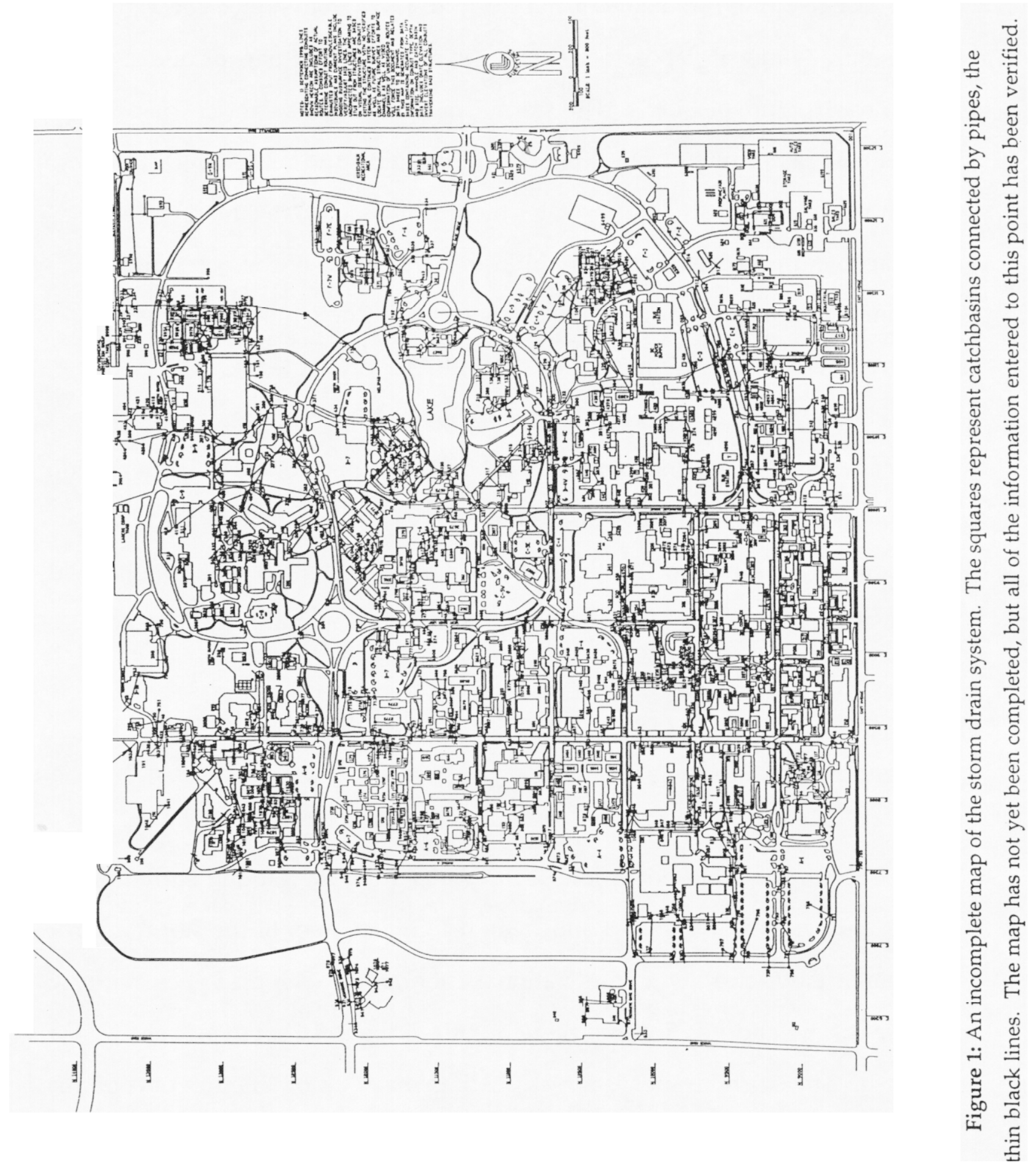


catchbasin. The pipes connect the catchbasins and the water flows downslope through this network of pipes. The pipes also flow into ditches and out to the arroyos.

The general flow of storm water through the system is from the southeast corner to the northwest corner of LLNL. There are two main points where storm water discharges from LLNL, Arroyo Seco and Arroyo Las Positas, located in the southwest corner and northwest corners of LLNL, respectively. The storm water from these two points joins with other flow and eventually discharges to the San Francisco Bay.

Why model the storm drain system?

A model of the storm drain system will provide valuable information about the system. The storm drain system is close to fifty years old. Over the fifty years that LLNL has been in existence, portions of the system have been built over, altered, or abandoned. Therefore, there is a great deal of uncertainty about the system. Developing this model will aid in gaining a better understanding of the state of the storm drain system.

The model will produce hydrographs, plots of outflow versus time, and/or pollutographs, plots of pollutant loading versus time, at select points throughout the system. These graphs will provide valuable information about the storm drain system for several reasons. Figure 2 shows a hydrograph and pollutograph produced by SWMM for an 80 minute storm of varying intensity. These graphs show several pieces of important information. The peak arrival time indicates the amount of time required for the peak flow or pollutant loading due to a storm to arrive at a point of 
interest. This ability to determine arrival times and peak values is important for several reasons, such as evaluation of the capacity of the storm drain system, and response time in the case of spills.
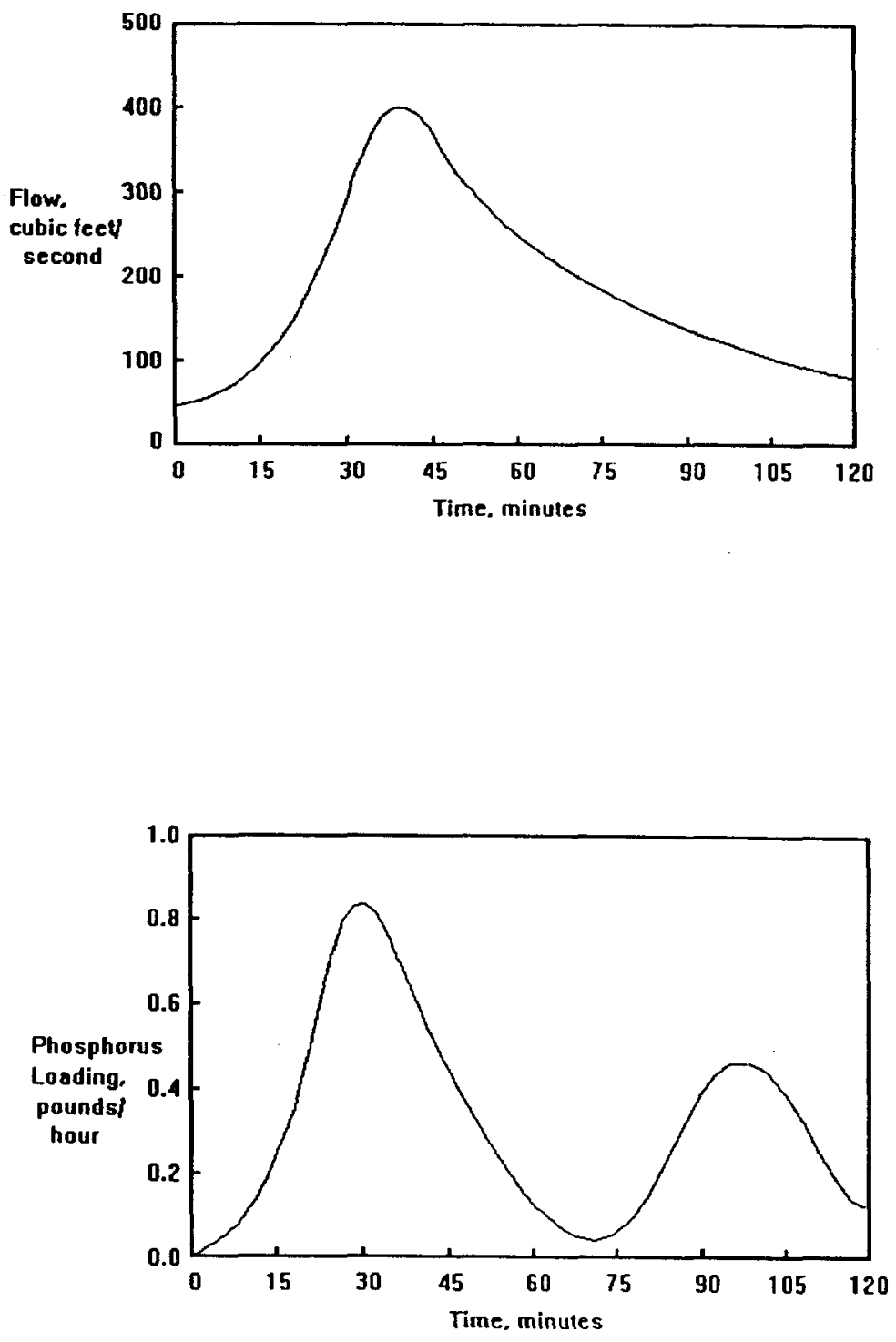

Figure 2: Sample of hydrograph and pollutograph produced by SWMM ${ }^{3}$ 
One important use for the information gained from hydrographs and pollutographs will be in evaluating best management practices (BMPs). As LLNL implements BMPs, it is anticipated that pollutant concentrations in the storm water will decrease. If this trend is not observed, it will indicate the need for more stringent BMPs or more inspections of BMPs. In the case of a spill that enters the storm drain system, the graph will indicate the response time that is available. This ability to respond to an accident with sufficient rapidity will ensure that LLNL remains in compliance, and protects the receiving waters that accept storm water runoff.

Currently, the Water Guidance and Monitoring Group monitors storm water quality and flow at several locations around LLNL, including the two discharge points. In recent years, there have been indications of a rising level of some contaminants in the runoff that is leaving LLNL. The model can be used to help identify the source of a contaminant, as well as discern the signal from the background noise. Similarly, the model will aid in spill traceback capabilities in the case of the detection of contamination leaving LLNL. By comparing possible scenarios for the source of contamination, the approximate location of the spill could be determined.

The graphs will also aid in evaluating the capacity of the pipes and culverts that make up the storm drain system at LLNL. The hydrographs will indicate the flow rate that results from storms of different intensities. Using this information, one can determine whether or not the storm drain system will have the capacity to handle the flow that results due to the storm. The model will also aid in evaluating proposed changes to the storm drain system, such as rerouting a pipe or adding a new catchbasin. 


\section{The USEPA Storm Water Management Model (SWMM)}

SWMM is the Stormwater Management Model, developed by the United States Environmental Protection Agency. SWMM has the capability to model flow through sewer systems, storage and treatment facilities as well as overland flow. The model uses different components, or blocks to model each of these different types of flow. For example, the runoff block models overland flow while the transport block models flow through pipes and channels.

SWMM is a FORTRAN- based program, and data is entered in each block in a free input format. The program provides template files for data entry, and values for each parameter are entered, each separated by a space. Figure 3 is an example of a runoff block program. The dollar symbol (\$) calls the block that is to be executed, as in this figure, the second line is \$RUNOFF. The lines with asterisks $\left(^{*}\right)$ at the beginning of the line are comment lines, and are ignored by the computer. The program in figure 3 represents a simple example of data entry to the SWMM model. Once data have been entered to the SWMM model, the data is run in the SWMM engine. The SWMM engine compiles the data and produces output graphs. These output graphs are the hydrographs and pollutographs that were discussed previously.

The runoff and transport blocks are being used to create the model of the LLNL storm drain system. The runoff block contains information about the storm duration and intensity and the surface that the rain flows across. The transport block contains information about the pipes and channels that the water flows through. Each of these blocks can be executed separately, or 


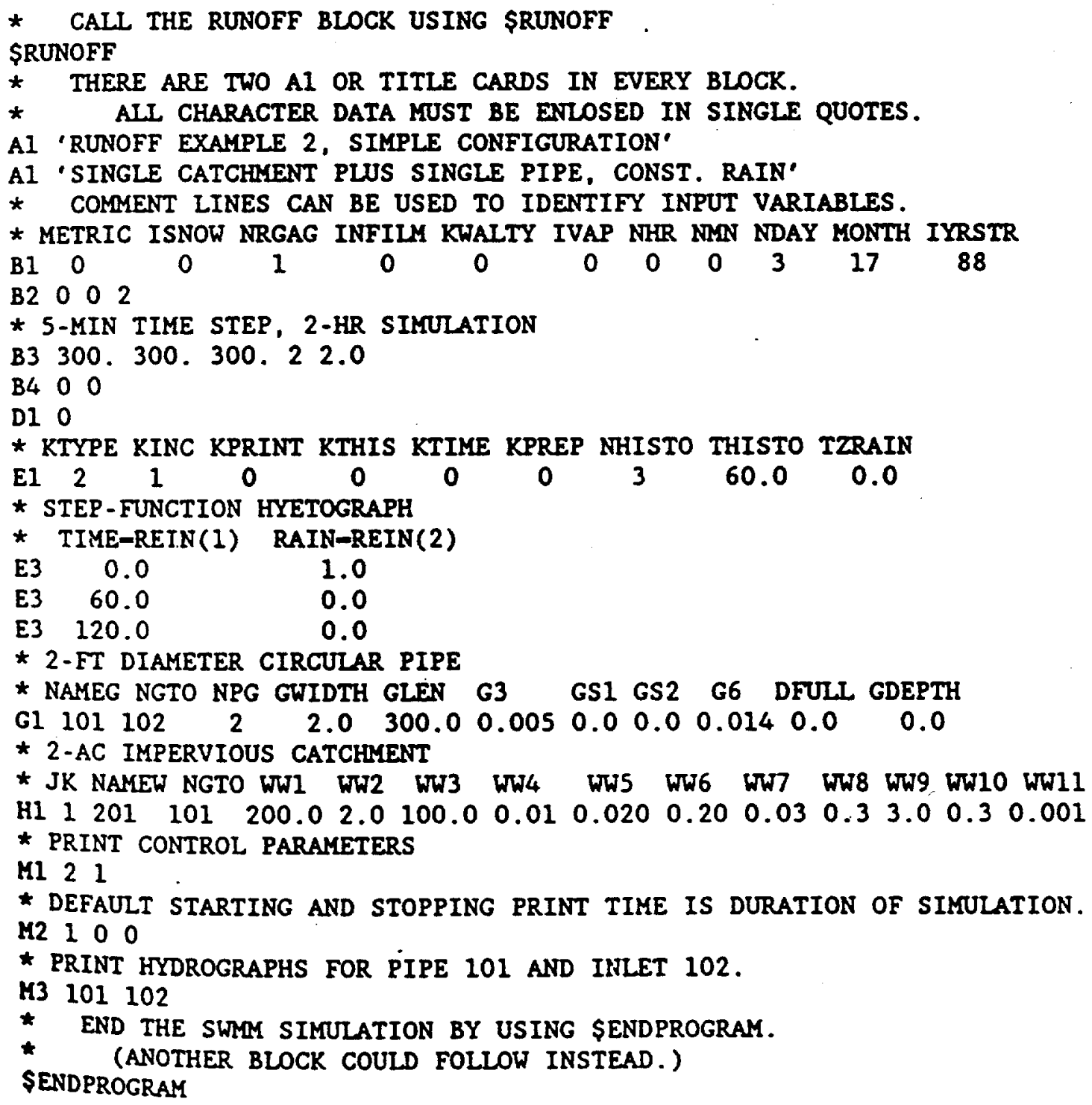

Figure 3: A sample of input to the runoff block. The runoff block contains subcatchment information as well as storm duration and intensity. 
they can be used in tandem to route the flow from the land to the pipe and channel network. For the LLNL storm drain model, these two blocks were linked. The flow in the runoff block enters a single catchbasin, this catchbasin is also entered to the pipe network in the transport block. The runoff then goes through both blocks through the interface of these catchbasins. In addition to containing common elements, the two block icons are connected on screen by an arrow.

The runoff block contains information about the runoff surfaces of LLNL. The Laboratory is divided into smaller drainage areas, known as subcatchments, for entry to runoff block. The division into subcatchments is based upon uniform characteristics such as surface slope and permeability. The following data are entered for each subcatchment: area, slope, and the following parameters: manning's roughness, infiltration parameters, and depression storage. For the first run of the model, the default values were used for these parameters, and they were considered to be uniform across the site. The entries for each subcatchment also indicate the destination for the overland flow from that subcatchment. In the LLNL model, the runoff enters a catchbasin. The catchbasin is then entered in the transport block.

The transport block contains information about the pipe and channel network. The transport block is prepared to receive data about each catchbasin and the pipes that are connected through that catchbasin. Each element is entered into the transport block with an identifying number that causes SWMM to recognize what type of element that it is. For example, all catchbasins are identified by the number 19 entered in the data row. The transport block contains the following information for each element: diameter, length, manning's roughness coefficient, slope, and depth and width if it is a channel. None of these parameters are entered if the element 
is a catchbasin or a manhole. The connection between the elements is indicated by entering the elements that lie directly upgradient of the element that is being entered.

\section{Gathering Data for the Model}

The runoff and transport blocks require data regarding the land surface and the pipes and channels. The data for the runoff block are obtained from topographic and site maps. The site plan was divided into approximately 75 subcatchments, and the area of each subcatchment was determined using a planimeter. The slope of each of these areas was determined from topographic maps. This information, in addition to manning's roughness coefficient, infiltration parameters, and depression storage values, was entered to the runoff block for each subcatchment. To begin the model, default values were used for the preceding parameters.

In general, the data for the transport block can be gathered from an upto-date map of the storm drain system. In the case of the LLNL storm drain system, the data on the storm drain map was questionable, and therefore the information needed to be gathered from other sources. The data for the transport block was gathered from field verifications and review of old site plans. The site plans show a specific area as it was built. These plans are considered more reliable than the current map because they are based upon post construction information. The information from the plans and field verifications was entered in the model as well as to a map of the storm drain system that is currently being created. 
The field verifications involved working in the field and recording information gathered at catchbasins. Figure 4 diagrams the information that was gathered at each catchbasin. The invert elevation is the distance from the bottom of the inside of the pipe to the top of the catchbasin. The rim elevation is the height of the catchbasin above sea level. A survey of all of the catchbasins at LLNL was recently completed, therefore this information is complete and up-to-date. If this information, in addition to the distance between two catchbasins that are connected, the slope of the pipe between these catchbasins can be determined. A spreadsheet was developed to determine this information for the LLNL storm drain system.

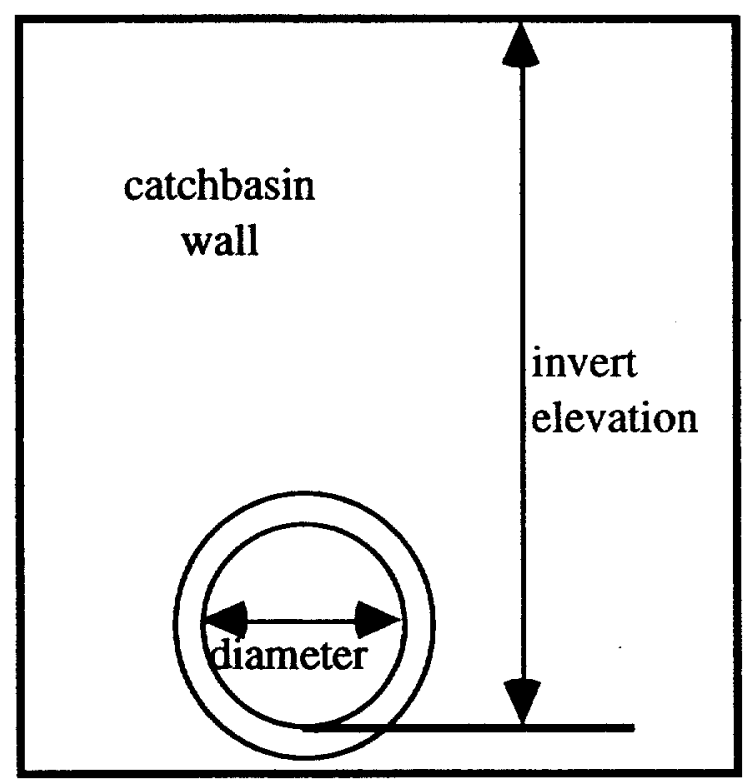

Figure 4: Diagram of the inside of a catchbasin indicating measurements taken for a field verification. 


\section{Results of the Model}

The model is not complete at this point. Portions of the laboratory have been modeled, including the western and northern perimeters and the southwestern corner. Hydrographs have been produced for these areas for a simulated storm event. The accuracy of these results will not be known until the storm drain map has been completed and the pipe connections have been validated.

Figure 5 shows a hydrographs produced by SWMM for the southwestern corner of the laboratory. These hydrographs were produced for a three and a half hour storm event of uniform intensity of 0.1 inches/hour. This graph shows the flow rate versus time where a pipe discharges into Arroyo Seco, location 100, and 1500 feet upgradient of this point, location 3. This graph is a good example of the information that SWMM output provides. The graph indicates the maximum flow rate that will result from this storm event. This information is useful in determining if the pipe or channel has adequate capacity to contain the resulting storm flow. The graph also provides important information about the timing of the storm flow. In the event of a spill into the storm drain system, the arrival time of the peak flow indicates the amount of time to respond to the spill. The graph also indicates the relative contribution of a single catchbasin to the flow into the Arroyo.

Pollutants have not been modeled yet. Up to four pollutants can be modeled in a single run for the transport block. Pollutants can include chemical compounds, biochemical oxygen demand, and suspended solids. Once the pollutant information is entered to the model, pollutographs can be generated at select points throughout the system. The pollutograph will 
indicate the concentration of a select contaminant in the storm water outflow. This information is extremely important for storm water monitoring and for evaluating the urgency of a spill to the storm drain system.

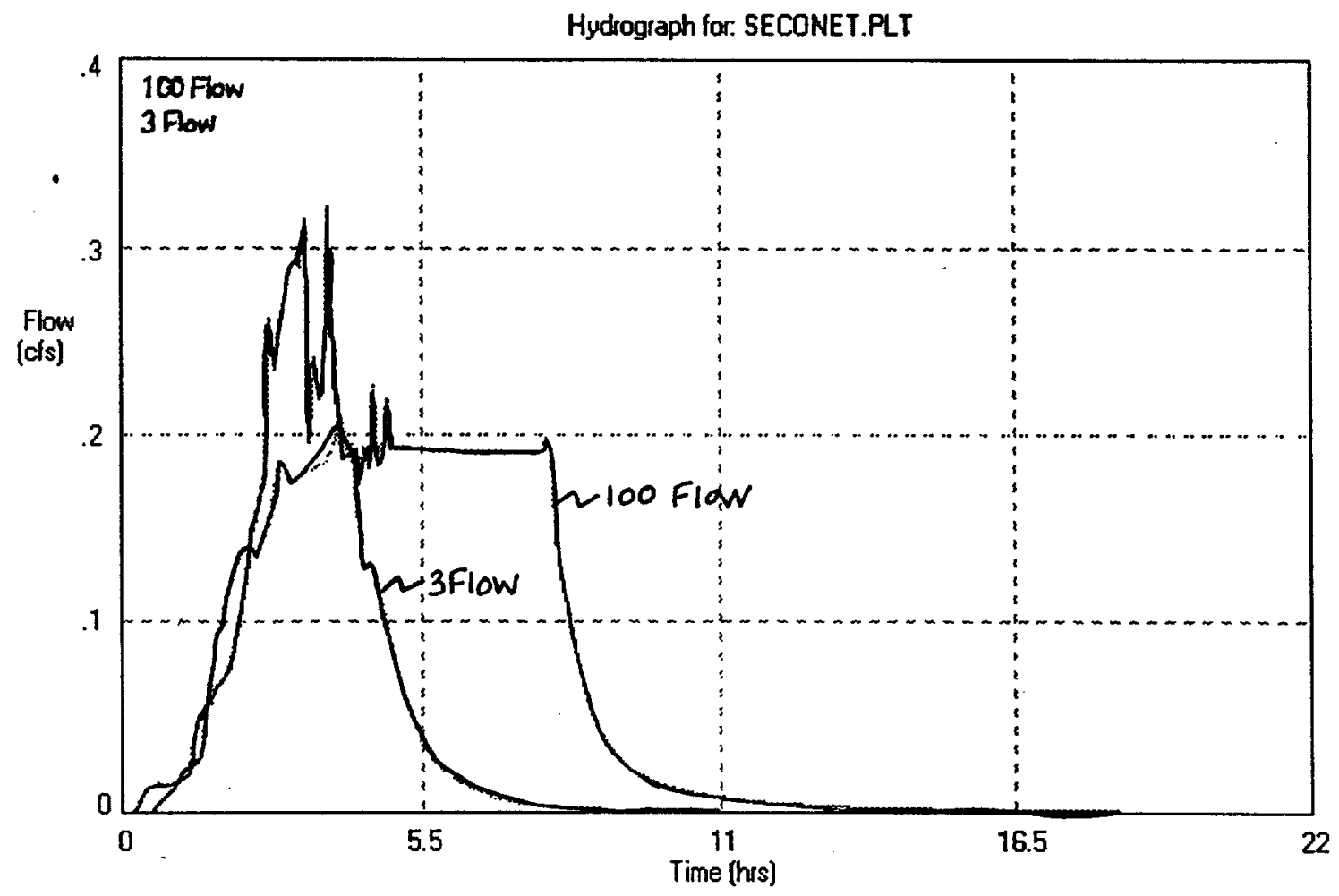

Figure 5: Hydrograph produced by SWMM for flow to Arroyo Seco

Location 100: Discharge to the Arroyo

Location 3: A catchbasin 1500 feet upgradient of the Arroyo

\section{Future Work}

In order to obtain a complete and accurate storm drain model, the storm drain map needs to be completed. To complete the map, field 
verifications need to be completed around the laboratory, and the data need to be entered to the model and to the map. More work can be done to make the model more accurate by replacing the parameters that have default values with values that are more accurate. One of the most important parameters that requires further research is the infiltration parameter. Once the data have been entered to the model and the storm drain system is accurately represented by the model. As soon as the model is producing accurate outflow graphs for the laboratory, further work can be done to add quality constituents to the model. 


\section{References}

[1] Huber, W.C., and R.E. Dickinson, Storm Water Management Model, Version 4: User's Manual. Environmental Protection Agency, Athens, Georgia, 1988.

[2] James, W., and W.R.C. James, PCSWMM Getting Started, 2nd Ed., Computational Hydraulics Inc., Guelph, Ontario, Canada, 1995.

[3] Nix, Stephen J., Urban Stormwater Modeling and Simulation, CRC Press, Inc., Boca Raton, Florida, 1994. 


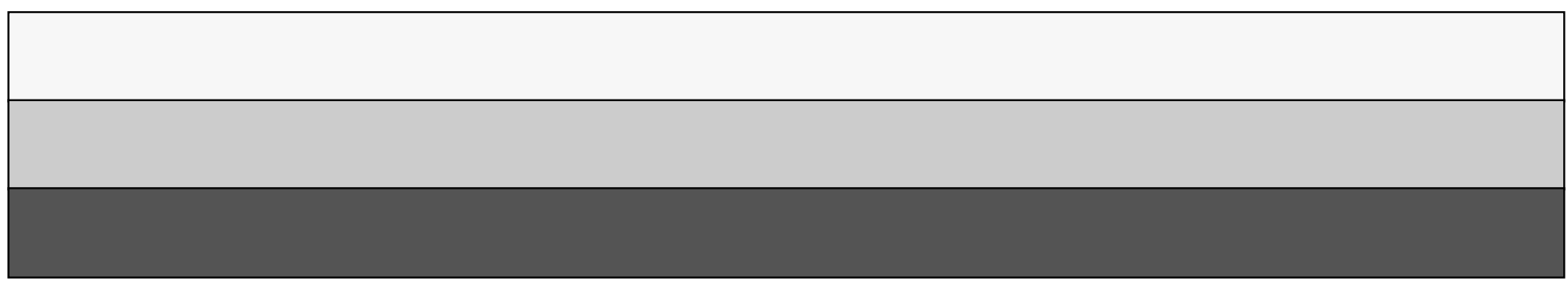

